University of Louisville

ThinkIR: The University of Louisville's Institutional Repository

Electronic Theses and Dissertations

7-2007

\title{
Fluid dynamics analysis of oscillating flow in petri dishes.
}

Jonathan Michael D. Thomas 1984-

University of Louisville

Follow this and additional works at: https://ir.library.louisville.edu/etd

\section{Recommended Citation}

Thomas, Jonathan Michael D. 1984-, "Fluid dynamics analysis of oscillating flow in petri dishes." (2007). Electronic Theses and Dissertations. Paper 1430.

https://doi.org/10.18297/etd/1430

This Master's Thesis is brought to you for free and open access by ThinkIR: The University of Louisville's Institutional Repository. It has been accepted for inclusion in Electronic Theses and Dissertations by an authorized administrator of ThinkIR: The University of Louisville's Institutional Repository. This title appears here courtesy of the author, who has retained all other copyrights. For more information, please contact thinkir@louisville.edu. 
FLUID DYNAMICS ANALYSIS OF OSCILLATING FLOW IN PETRI DISHES

\author{
By \\ Jonathan Michael D Thomas \\ B.S., University of Louisville, 2006
}

\begin{abstract}
A Thesis
Submitted to the Faculty of the University of Louisville

in Partial Fulfillment of the Requirements

for the Professional Degree
\end{abstract}

MASTER OF ENGINEERING

Department of Chemical Engineering

July 2007 


\section{FLUID DYNAMICS ANALYSIS OF OSCILLATING FLOW IN PETRI DISHES}

Submitted by:

Jonathan Michael D Thomas

A Thesis Approved On

(Date)

by the Following Reading and Examination Committee:

Dr. R. Eric Berson, Thesis Director

Dr. Walden L. S. Laukhuf

Dr. M. Keith Sharp 


\section{ACKNOWLEDGMENTS}

First I would like to thank God for all his help through my research and writing.

Without my parents' encouragement and direction I would have never been in the position to receive a Masters degree.

Dr. Berson's guidance has helped immensely with my thesis progress and my growth as a researcher.

Nate Johnson was a life-saver on those days when Adelie decided to have a mind of its own. 


\begin{abstract}
Orbital shakers are commonly used in the cell culture industry due to their ease of use, but the fluid dynamics of this system have not been extensively modeled as the system is difficult to quantify analytically. It is desirable to understand how cultured cells respond to fluid forces as the result of motion of a dish on an orbiting platform under varying flow conditions. Wall shear stresses are commonly accepted as the primary influence affecting characteristics of anchored cells subjected to fluid flow. Cells become aligned and elongated with the direction of flow when shear stresses are experienced.
\end{abstract}

In this work, FLUENT, a commercial CFD package is utilized to model fluid behavior in these dishes. To simplify computational effort, the investigation is reduced to the study of the following three dimensionless parameters: the Stokes Number, the Froude Number, and the Slope Ratio. It is desirable to identify how the fluid behaves at low and high values for each of these parameters and where the transition from low to high occurs for each of these values. The study yielded the following findings. The Stokes Transition occurs at a Stokes Number of $4.1 \pm 0.1$ for a Froude Number of 1.0 and a Slope Ratio of 1.0 and results in a localized increase in the slope of maximum WSS vs. Stokes Number. The Froude Transition occurs at a Froude Number of $0.25 \pm 0.05$ for a Stokes Number of 5.0 and a Slope Ratio of 1.0 and results in a localized increase in the slope of maximum WSS vs. Froude Number. The Slope Transition occurs at a Slope Ratio of $1.25 \pm 0.15$ for a Stokes Number of 5.0 and a Froude Number of 1.0 and results in a localized increase in the slope of maximum WSS vs. Slope Ratio. 


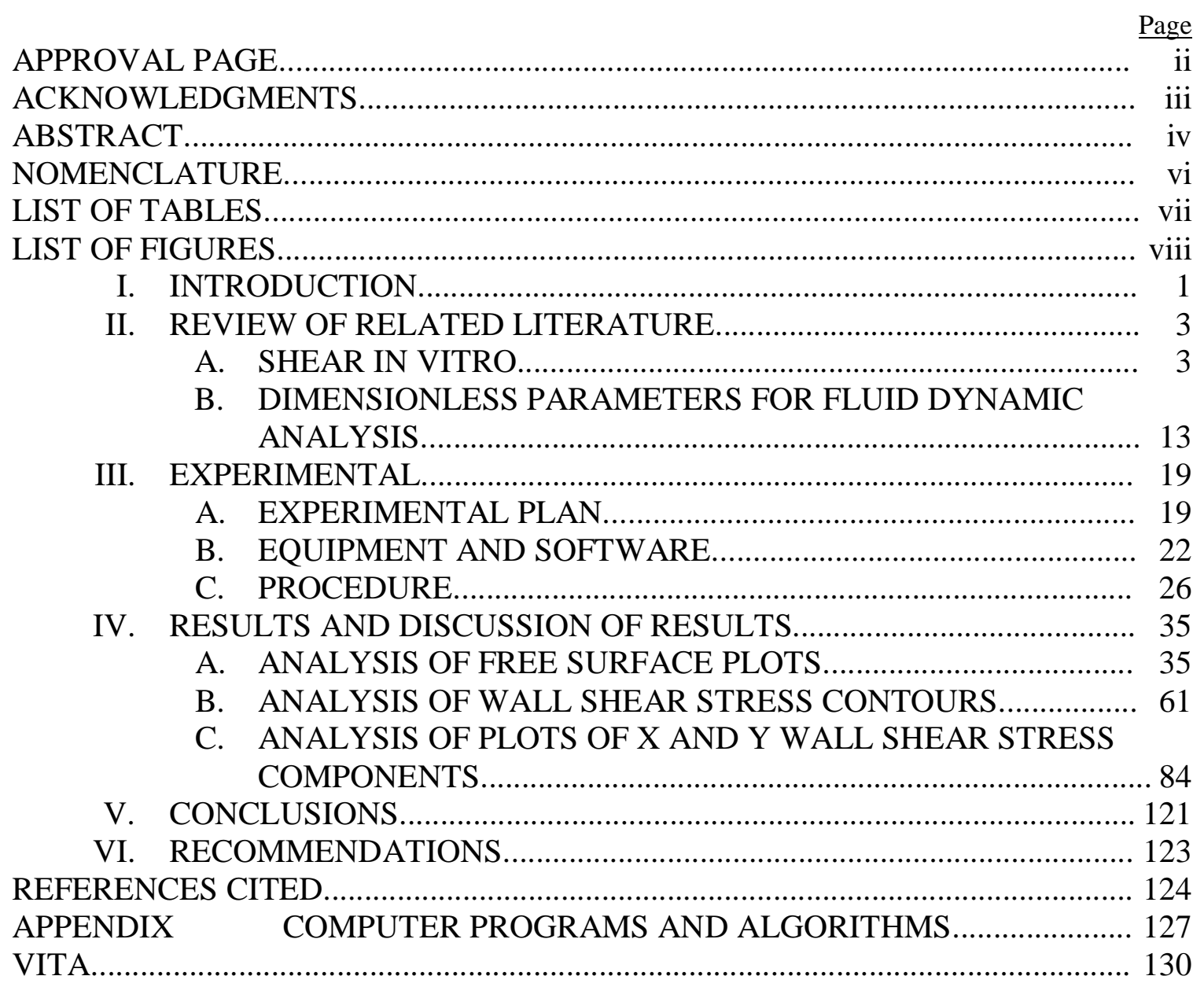




\section{NOMENCLATURE}

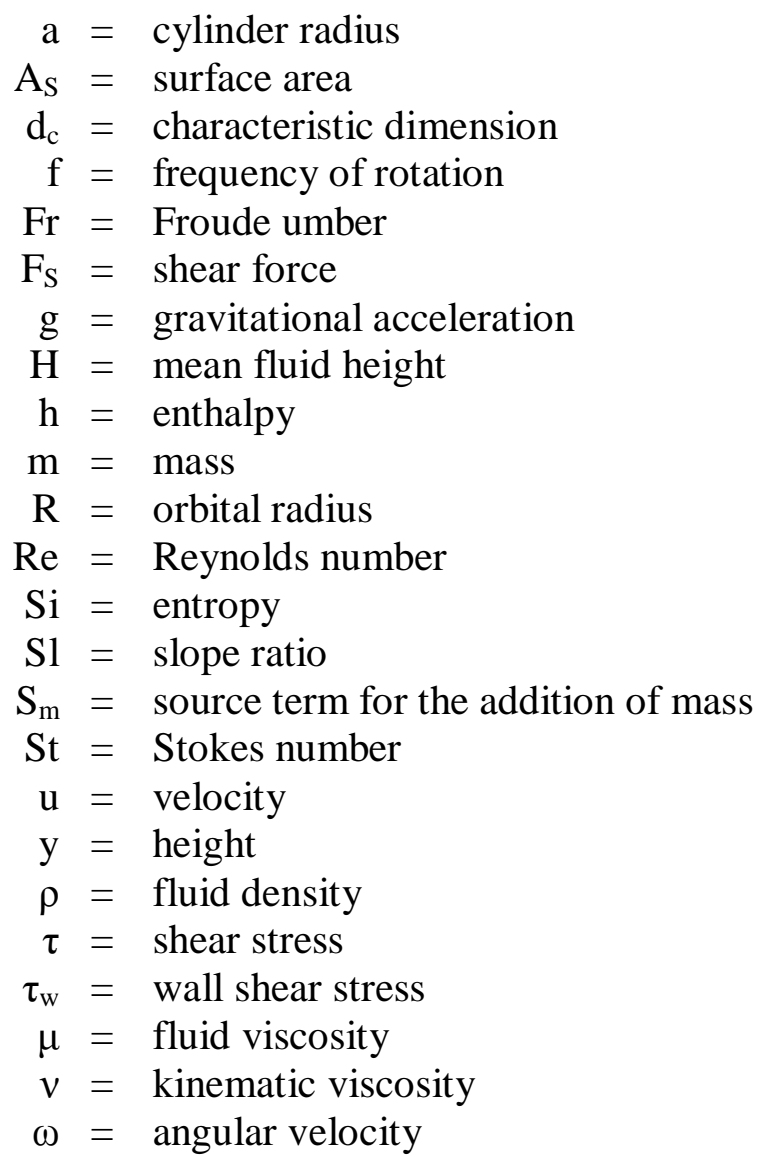




\section{LIST OF TABLES}

TABLE I - Cases for Varying Stokes Number............................................... 21

TABLE II - Cases for Varying Froude Number............................................... 21

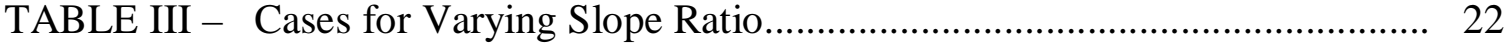

TABLE IV - Phase Lag Observed vs. Stokes Numbers........................................... 43

TABLE V - Maximum Bottom Wall Shear Stress vs. Stokes Numbers.................... 61

TABLE VI - Maximum Bottom Shear Stress vs. Froude Numbers........................... 70

TABLE VII - Maximum Wall Shear Stress vs. Slope Ratios.................................... 78 


\section{LIST OF FIGURES}

FIGURE 1 - Dish with Seven Parameters Labeled............................................... 14

FIGURE 2 - Cube of Dimensionless Parameters...................................................... 19

FIGURE 3 - Region Adaptation Menu............................................................ 31

FIGURE 4 - Solution Controls Menu.............................................................. 32

FIGURE 5 - Contours Window..................................................................... 34

FIGURE 6 - $\quad$ Grid for Determining Phase Lag......................................................... 36

FIGURE 7 - Stokes Number 1.0 Free Surface Plot................................................... 37

FIGURE 8 - Stokes Number 2.0 Free Surface Plot................................................. 37

FIGURE 9 - $\quad$ Stokes Number 3.0 Free Surface Plot............................................... 38

FIGURE 10 - Stokes Number 4.0 Free Surface Plot................................................... 38

FIGURE 11 - Stokes Number 5.0 Free Surface Plot................................................. 39

FIGURE 12 - Stokes Number 10 Free Surface Plot............................................... 39

FIGURE 13 - Stokes Number 4.2 Free Surface Plot............................................... 40

FIGURE 14 - Stokes Number 4.4 Free Surface Plot.................................................... 41

FIGURE 15 - Stokes Number 4.6 Free Surface Plot............................................... 41

FIGURE 16 - Stokes Number 4.8 Free Surface Plot.................................................... 42

FIGURE 17 - Froude Number 0.2 Free Surface Plot.................................................. 44

FIGURE 18 - Froude Number 0.3 Free Surface Plot................................................... 45

FIGURE 19 - Froude Number 0.1 Free Surface Plot................................................ 46

FIGURE 20 - Froude Number 0.12 Free Surface Plot................................................... 47

FIGURE 21 - Froude Number 0.14 Free Surface Plot................................................. 47

FIGURE 22 - Froude Number 0.16 Free Surface Plot............................................... 48

FIGURE 23 - Froude Number 0.18 Free Surface Plot............................................. 48

FIGURE 24 - Froude Number 0.4 Free Surface Plot................................................. 50

FIGURE 25 - Froude Number 0.5 Free Surface Plot................................................. 50

FIGURE 26 - Froude Number 0.7 Free Surface Plot.................................................. 51

FIGURE 27 - Froude Number 1.0 Free Surface Plot.................................................. 51

FIGURE 28 - Slope Ratio 0.5 Free Surface Plot......................................................... 53

FIGURE 29 - Slope Ratio 2.0 Free Surface Plot..................................................... 53

FIGURE 30 - Slope Ratio 0.8 Free Surface Plot...................................................... 54

FIGURE 31 - Slope Ratio 1.1 Free Surface Plot.................................................... 55

FIGURE 32 - Slope Ratio 1.4 Free Surface Plot........................................................ 55

FIGURE 33 - Slope Ratio 1.7 Free Surface Plot......................................................... 56

FIGURE 34 - Slope Ratio 0.8 Back Side Free Surface Plot....................................... 57

FIGURE 35 - Slope Ratio 1.1 Back Side Free Surface Plot........................................ 58

FIGURE 36 - Slope Ratio 0.1 Front Side Free Surface Plot....................................... 59

FIGURE 37 - Slope Ratio 0.1 Back Side Free Surface Plot......................................... 59

FIGURE 38 - Slope Ratio 0.2 Front Side Free Surface Plot........................................ 60

FIGURE 39 - Slope Ratio 0.2 Back Side Free Surface Plot......................................... 60

FIGURE 40 - Maximum Bottom Wall Shear Stress versus Stokes Number............... 63

FIGURE 41 - Stokes Number Data Comparison to Equations..................................... 63

FIGURE 42 - Modified Stokes Number Data Comparison to Equations..................... 64 
FIGURE 43 - Stokes Number 1.0 Wall Shear Stress Contour..................................... 66

FIGURE 44 - Stokes Number 2.0 Wall Shear Stress Contour..................................... 66

FIGURE 45 - Stokes Number 3.0 Wall Shear Stress Contour..................................... 66

FIGURE 46 - Stokes Number 2.0 Free Surface Plot with Low Froude Effect............ 67

FIGURE 47 - Stokes Number 3.0 Free Surface Plot with High Froude Effect........... 67

FIGURE 48 - Stokes Number 4.0 Wall Shear Stress Contour...................................... 68

FIGURE 49 - Stokes Number 4.2 Wall Shear Stress Contour.................................... 68

FIGURE 50 - Stokes Number 4.4 Wall Shear Stress Contour..................................... 68

FIGURE 51 - Stokes Number 4.6 Wall Shear Stress Contour.................................... 69

FIGURE 52 - Stokes Number 4.8 Wall Shear Stress Contour................................... 69

FIGURE 53 - Stokes Number 5.0 Wall Shear Stress Contour..................................... 69

FIGURE 54 - Stokes Number 10 Wall Shear Stress Contour...................................... 70

FIGURE 55 - Maximum Bottom Shear Stress versus Froude Number...................... 72

FIGURE 56 - Froude Number Data Comparison to Equations.................................... 72

FIGURE 57 - Froude Number 0.1 Wall Shear Stress Contour.................................... 74

FIGURE 58 - Froude Number 0.12 Wall Shear Stress Contour.................................. 74

FIGURE 59 - Froude Number 0.14 Wall Shear Stress Contour................................. 74

FIGURE 60 - Froude Number 0.16 Wall Shear Stress Contour................................. 75

FIGURE 61 - Froude Number 0.18 Wall Shear Stress Contour................................. 75

FIGURE 62 - Froude Number 0.2 Wall Shear Stress Contour................................... 75

FIGURE 63 - Froude Number 0.3 Wall Shear Stress Contour.................................... 76

FIGURE 64 - Froude Number 0.4 Wall Shear Stress Contour................................... 76

FIGURE 65 - Froude Number 0.5 Wall Shear Stress Contour................................... 76

FIGURE 66 - Froude Number 0.7 Wall Shear Stress Contour.................................... 77

FIGURE 67 - Froude Number 1.0 Wall Shear Stress Contour..................................... 77

FIGURE 68 - Maximum Wall Shear Stress versus Slope Ratio................................... 79

FIGURE 69 - Froude Number Data Comparison to Equations................................... 79

FIGURE 70 - Slope Ratio 0.1 Wall Shear Stress Contour.......................................... 81

FIGURE 71 - Slope Ratio 0.2 Wall Shear Stress Contour........................................ 81

FIGURE 72 - Slope Ratio 0.5 Wall Shear Stress Contour.......................................... 82

FIGURE 72 - Slope Ratio 0.8 Wall Shear Stress Contour........................................... 82

FIGURE 74 - Slope Ratio 1.1 Wall Shear Stress Contour......................................... 82

FIGURE 75 - Slope Ratio 1.4 Wall Shear Stress Contour.......................................... 83

FIGURE 76 - Slope Ratio 1.7 Wall Shear Stress Contour............................................ 83

FIGURE 77 - Slope Ratio 2.0 Wall Shear Stress Contour......................................... 83

FIGURE 78 - Stokes Number 2.0 Plot of X and Y WSS Components and X and Y

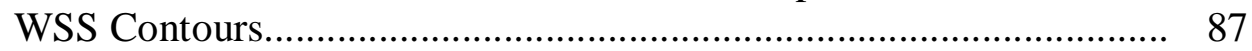

FIGURE 79 - Stokes Number 3.0 Plot of X and Y WSS Components and X and Y WSS Contours...................................................................... 88

FIGURE 80 - Stokes Number 4.0 Plot of X and Y WSS Components and X and Y

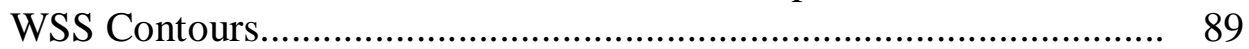

FIGURE 81 - Stokes Number 4.2 Plot of X and Y WSS Components and X and Y

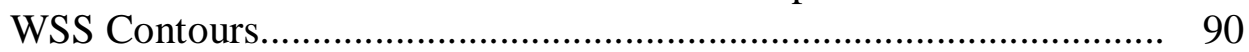

FIGURE 82 - Stokes Number 4.4 Plot of X and Y WSS Components and X and Y WSS Contours 
FIGURE 83 - Stokes Number 4.6 Plot of X and Y WSS Components and X and Y WSS Contours

FIGURE 84 - Stokes Number 4.8 Plot of X and Y WSS Components and X and Y WSS Contours.

FIGURE 85 - Stokes Number 5.0 Plot of X and Y WSS Components and X and Y WSS Contours

FIGURE 86 - Stokes Number 10 Plot of X and Y WSS Components and X and Y WSS Contours.

FIGURE 87 - Stokes Number 5.0 with no Slope Effect......................................... 96

FIGURE 88 - Stokes Number 10 with Slope Effect.............................................. 96

FIGURE 89 - Froude Number 0.1 Plot of X and Y WSS Components and X and Y WSS Contours

FIGURE 90 - Froude Number 0.12 Plot of X and Y WSS Components and X and Y WSS Contours

FIGURE 91 - Froude Number 0.14 Plot of X and Y WSS Components and X and Y WSS Contours

FIGURE 92 - Froude Number 0.16 Plot of X and Y WSS Components and X and Y WSS Contours

FIGURE 93 - Froude Number 0.18 Plot of X and Y WSS Components and X and Y WSS Contours

FIGURE 94 - Froude Number 0.2 Plot of X and Y WSS Components and X and Y WSS Contours.

FIGURE 95 - Froude Number 0.3 Plot of X and Y WSS Components and X and Y WSS Contours

FIGURE 96 - Froude Number 0.4 Plot of X and Y WSS Components and X and Y WSS Contours

FIGURE 97 - Froude Number 0.5 Plot of X and Y WSS Components and X and Y WSS Contours

FIGURE 98 - Froude Number 0.1 with Stokes Effect Present.................................. 108

FIGURE 99 - Froude Number 1.0 with no Slope Ratio Effect Present....................... 108

FIGURE 100 - Froude Number 0.7 Plot of X and Y WSS Components and X and Y WSS Contours.

FIGURE 101 - Froude Number 1.0 Plot of X and Y WSS Components and X and Y WSS Contours

FIGURE 102 - Slope Ratio 0.1 Plot of X and Y WSS Components and X and Y WSS Contours

FIGURE 103 - Slope Ratio 0.2 Plot of X and Y WSS Components and X and Y WSS Contours

FIGURE 104 - Slope Ratio 0.5 Plot of X and Y WSS Components and X and Y WSS Contours

FIGURE 105 - Slope Ratio 0.8 Plot of X and Y WSS Components and X and Y WSS Contours.

FIGURE 106 - Slope Ratio 0.5 with No Visible Stokes Effect......

FIGURE 107 - Slope Ratio 0.8 with Visible Stokes Effect

FIGURE 108 - Slope Ratio 1.1 Plot of X and Y WSS Components and X and Y WSS Contours 
FIGURE 109 - Slope Ratio 1.4 Plot of X and Y WSS Components and X and Y WSS Contours............................................................................. 118

FIGURE 110 - Slope Ratio 1.7 Plot of X and Y WSS Components and X and Y WSS Contours.

FIGURE 111 - Slope Ratio 2.0 Plot of X and Y WSS Components and X and Y

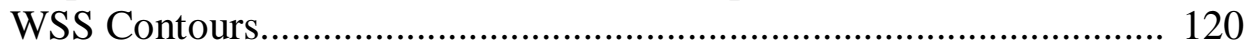

FIGURE 112 - Example UDF................................................................... 128

FIGURE 113 - Example Batch File............................................................... 128

FIGURE 114 - Example Input File......................................................................... 129 


\section{INTRODUCTION}

The effects of hemodynamic forces on cellular responses have been studied for at least three decades, but the mechanisms linking cause and effect have not been concretely identified. Hemodynamics is the study of the properties and flow of blood. Wall shear stresses are commonly accepted as the primary influence affecting characteristics of anchored cells subjected to fluid flow. For example, endothelial cells, lining the interior walls of arteries and veins, experience shear exerted by the flow of blood and become aligned and elongated with the direction of flow and undergo other physiological and biochemical changes (Levesque and Nerem, 1985). The realization of the relationship between hemodynamic forces on the endothelium and the origins of atherosclerosis and

vascular pathology, in general, has led to considerable attention focusing on the effects of these forces on cellular responses. Detailed, accurate information about the fluid forces acting on cells must be known in order to understand the cause and effect relationship between shear stresses and endothelial responses.

The system of study involves endothelial cell cultures grown in a Petri dish oscillating on a shaker platform. Orbital shakers are commonly used in the cell culture industry due to their ease of use, but the fluid motion inside such dishes have not been accurately modeled as the system is difficult to quantify analytically. Furthermore, it is desirable to understand how cultured cells respond to fluid forces on an orbiting platform under varying flow conditions. These fluid forces are the combination of many properties of the system including: radius of orbit, orbital speed, dish diameter, viscosity, fluid height, density, and gravity. It would take an extensive endeavor to identify how 
each property affects fluid forces. To reduce the computational effort the effects of four dimensionless parameters, which incorporate these seven variables, on the behavior of the fluid forces can be more easily obtained. These parameters are the Stokes Number, the Slope Ratio, the Froude Number, and the Reynolds Number. Taking flow as laminar removes Reynolds Number as a variable and reduces the system to three dimensionless parameters. From a fluid dynamics standpoint, it is desirable to know how the fluid behaves at low and high values for each of these parameters and where the transition from high to low for each of these values occurs. At the transition point, each of the parameters has a unique effect that can be visually observed. The objective of this work is to determine the transition point for these three parameters, and to define the effect the transition has on shear in the dish. To establish where the transition occurs, each of these parameters is varied from high or low values while the other two parameters are kept constant at a medium value. By studying a range from low to high for each parameter, it is possible to know how shear stress characteristics are affected at each region, as well as at the transition.

As of yet, little effort has been made to experimentally determine fluid forces in an oscillating dish. Until now, because of the complexities in developing an accurate quantative analysis, only oversimplified point functions have been used. To find the desired results, a computational model is created. Models are created here through the use of commercial computational fluid dynamics (CFD) software. The preprocessor GAMBIT is used to create the cylindrical-shaped geometry, and FLUENT solves the Navier-Stokes equations to create a complete representation of the flow field inside the orbiting dishes including the wall shear stresses imposed on cells in culture. 


\section{REVIEW OF RELATED LITERATURE}

\section{A. Shear in Vitro}

\section{Definition of Shear Stress}

In Fluid Dynamics, shear stress is an important property. Shear stress is directly related to velocity. McCabe et al. (2001) show that when a fluid flows past a wall, the fluid sticks to the wall at their interface. This results in a velocity of zero at the interface of the wall and fluid. At distances from the wall, the velocity is not zero, however, and hence variations must occur in velocity. This results in a velocity field in the fluid where the velocity at a given point is a function of both location and time. The velocity gradient is the reciprocal of the slope of the velocity field. The local velocity gradient is also called the shear rate. Velocity gradient is defined as the change in velocity over the change in distance or:

$$
\frac{d u}{d y}=\lim _{\Delta y \rightarrow 0} \frac{\Delta u}{\Delta y}
$$

A fluid resists shear so a shear force must exist wherever there is a shear rate. Shear forces act tangential to the plane in which the shear rate develops. Shear stresses are more commonly described than shear forces. Shear stress differs from normal stress in that shear stress is parallel to a plane, where normal stress is perpendicular to the plane. Since shear stress varies with distance, a field is created that also varies with distance and time. Shear stress $(\tau)$ is defined as the force per unit area of the shearing plane or: 


$$
\tau=\frac{F_{S}}{A_{S}}
$$

where $F_{S}$ is the shear force and $A_{S}$ is the area of the plane. Bird et al. (2002) show that equation (2) can be rearranged by substituting the velocity gradient as:

$$
\tau_{x y}=-\mu \frac{d u_{x}}{d y}
$$

This equation is known as Newton's Law of Viscosity.

\section{Methods to Determine Shear in a Laboratory}

A number of studies have been conducted in vitro over a cultured layer of endothelial cells using experimental apparatuses that can provide accurately quantifiable shear stress. A common method involves inducing flow in a chamber between two parallel plates. For this case, the width and length of the plates are substantially larger than the gap between them. Additionally, the flow chamber design provides steady, uniform laminar flow. Levesque and Nerem (1985) investigated the response of cultured bovine aortic endothelial cells subjected to shear stresses of 10,30 , and $85 \frac{d y n e s}{\mathrm{~cm}^{2}}$ for up to 24 hours using a parallel plate channel flow device. They discovered that $10 \frac{\text { dynes }}{\mathrm{cm}^{2}}$ had little effect on elongation and orientation of cells while 30 and $85 \frac{\text { dynes }}{\mathrm{cm}^{2}}$ had a significant effect. By knowing the flow rate and gap between the plates, the authors 
developed an equation for wall shear stress in the parallel plate channel flow device as follows:

$$
\tau_{\omega}=\frac{6 \mu^{2}}{\rho H^{2}} \operatorname{Re}=\frac{6 \mu u}{H}
$$

This equation assumes that viscosity and density are that of water. Four key findings resulted from the experiments: endothelial cells orient with the flow direction under the influence of shear stress, a shorter time is required for cell elongation than cell alignment with flow, the degree of alignment is closely related to cell shape, and endothelial cells become more elongated when introduced to greater shear stresses.

Another method for determining shear stress entails generating a rotating flow field over a layer of cells attached to base of each dish. When using this scheme, either a cone or a flat plate rotates over a stationary surface where the layer of cells resides. Ley et al. (1989) show that in a plate and cone apparatus, a secondary flow is induced on the primary shear field. The cause of secondary flow is centrifugal forces which produce a fluid movement toward center at the bottom of the well, and outward movement at the cone surface. Wall shear stress is easily calculated for the cone-and-plate apparatus since the rotation speed of the cone or flat plate and the length of the gap between the rotating and stationary surfaces are known. The equation the authors show is:

$$
\tau(a)=\frac{2 \pi a f \mu}{H}
$$


where $\tau(\mathrm{a})$ is the shear stress at a radius, a, of the cone and $\mathrm{H}$ is the fluid height.

The parallel plate channel flow device and cone-and-plate device described

provide an accurate means of supplying pre-determined shear stresses to a fixed layer of cells, but they are limited in that they only provide steady flows. They are further limited in that experiments must be performed successively as opposed to concurrently unless multiple devices are present.

Another apparatus for inducing flow on endothelial cells is the shaker flask. The shaker flask contains a suspended layer of cells with a magnetic bar that is agitated using a magnetic stirrer. Saarinen et al. (1995) compared production of recombinant secreted alkaline phosphatase protein in virally infected insect cells in a shaker flask to that in a rotating-wall vessel bioreactor. They showed that higher cell viability was detected in cultures grown in the rotating-wall vessel bioreactor compared to the shaker flask. The authors attributed this effect to lower shear stresses present in the rotating-wall vessel bioreactor. Lower shear stresses should result in less damage to cell membranes resulting in a longer time until membrane integrity is diminished. Elias et al. (1995) conducted experiments with a shaker flask to determine the effect of turbulent shear stress. They used a Laser Doppler Anemometer (LDA) to measure the shear stress in turbulent flow over a suspension of KG-1 cells derived from human leukemia. Before the study shear stress had not been quantified in a spinner flask. The authors found that varying agitation speed by up to 7.5 rotations per second did not damage the cells. However above 2.5 rotations per second, the cells became unable to proliferate further.

An apparatus widely used for providing fluid motion to endothelial cells is the orbital shaker platform. Orbital shakers are prevalent in the cell culture industry because 
of their simplicity of use. Additionally, they are ideal for simultaneous cell culture experiments. The most important aspect is that orbital shakers provide oscillatory flow, similar to that experienced by pulsing fluid movement in the human vasculature system.

Despite their popularity and simplicity of use, few researchers have attempted to utilize the orbiting shaker as a means for correlating shear stresses to cellular responses. This is due to the complexity of accurately calculating wall shear stresses exerted by the fluid in this configuration. The movement of fluid in a cylinder that derives its motion from an orbiting shaker platform is oscillatory in nature. A wave develops whose peak rotates around the cylinder at an angular velocity corresponding to the orbital velocity of the cylinder. Depending on the properties of the fluid and the relative forces, the rotating wave may or may not be in phase with the orbiting motion of the cylinder. Simplified means have been used in estimating the magnitude of the shear by those that have attempted to correlate shear stress in a shaker flask to cellular responses.

Ley et al. (1989) investigated shear-dependent adhesion of human polymorphonuclear neutrophil granulocytes (PMN) to endothelial cells in both orbital shaker and cone-and-plate experiments. They conducted experiments using human umbilical vein endothelial cells (HUVECs) and on plastic dishes coated with bovine serum albumin (BSA). They found that an estimated shear stress of $1.5 \frac{\text { dynes }}{\mathrm{cm}^{2}}$ reduced PMN adhesion from $36 \%$ to $21 \%$ on HUVECs and from $59 \%$ to $35 \%$ on BSA. The authors further used Dextran Sulphate as an inhibitor to PMN adhesion. At a concentration of $1 m g * m l^{-1}$, an inhibition of $72 \%$ was achieved on HUVECs and $76 \%$ was achieved on BSA. Dextran Sulphate showed no effects without shear stress present. To simplify their findings, the shear stresses they reported in the orbital shaker 
experiments were an estimate of the maximum wall shear stress at the bottom of the cylinder:

$$
\tau_{w}=a \sqrt{\rho \mu(2 \pi f)^{3}}
$$

where $\tau_{\omega}$ is the maximum wall shear stress on the bottom of the cylinder, a is the radius of the cylinder, $\rho$ is the fluid density, $\mu$ is the fluid viscosity, and $\mathrm{f}$ is the frequency of rotation. No derivation or reference of the origin of Equation (6) was provided by the authors. A surprising aspect of the equation is the fact that it does not include a term for the fluid height which is typically essential for a shear stress calculation as seen in Equation (3). However, the authors included a term to account for fluid height in their calculation of shear stress for the cone-and-plate experiments in Equation (5).

When Stokes second problem is extended to an orbital system, an equation develops identical to Equation (6) (Sharp, 2006). However, as derived for Stokes second problem, Equation (6) does not represent maximum shear stress as reported by Ley et al. (1987) but is for a constant magnitude of shear stress that applies across the central part of the dish. Furthermore, this derivation limits the equation to the conditions of high Stokes Number, low Froude Number, low Slope Ratio and low Reynolds Number. They also show a derivation of an equation for wall shear stress in quasi-steady orbital flow in a dish:

$$
\tau_{w}=\rho R \omega^{2} h
$$


As with Equation (6), Equation (7) also applies to the center of the dish, however it does contain a term for fluid height common with most shear equation. Equation (7) is limited to the conditions of low Stokes Number, low Froude Number, low Slope Ratio and low Reynolds Number.

Kraiss et al. (2000) and Kraiss et al. (2003) used Equation (6) in studying the mechanisms by which fluid flow regulates E-selectin protein production and activates p70/p85 S6 kinase in human endothelial cells. The authors claim that there is a similar reduction in E-selectin protein expression when comparing the endothelial response produced by oscillating fluid motion in an orbital shaker to the endothelial response produced by laminar fluid motion produced in a cone-and-plate apparatus. The lack of a quantitative comparison between the two methods could be due to the complication in comparing maximum wall shear stress, their only available estimate of a shear stress in oscillating flow, to an average shear stress for steady flow.

Equation (6) is also employed by Haga et al. (2003) when studying the effects of oscillatory shear stress on smooth muscle cell proliferation and Akt phosphorylation in an orbital shaker. They obtained unexpected results in that proliferation of smooth muscle cells increased in response to oscillatory shear stress; Sterpetti et al. (1993) and Ueba et al. (1997) had earlier reported that steady shear stress inhibited cell proliferation. Haga et al. attributed the disparity in these findings to the use of oscillatory versus steady flow, as had been previously used. They stated that oscillatory shear stress is likely a more physiological relevant model since it more closely models unsteady blood flow in vivo. They concluded that additional studies are needed to concretely define the variation between oscillatory and steady shear stress effects on smooth muscle cells. 
Dardik et al. (2005) also used Equation (6) in a study of differential effects of orbital and laminar shear stress. Believing different effects occurred between orbit shear stresses and laminar steady-flow shear stress, they conducted experiments on endothelial cells using both an orbital shaker apparatus and a parallel plate channel flow device. They found that orbital shear stress increased endothelial cell proliferation by $29 \%$, while a 38\% decrease in proliferation was discovered for laminar steady-flow shear stress. Furthermore, they found that the cells on the periphery of the dish showed a similar alignment to those seen in the laminar shear stress, whereas the cells were unaligned at the center of the dish. Further study found an average shear stress of $11 \frac{\text { dynes }}{\mathrm{cm}^{2}}$ at the periphery and $5 \frac{\text { dynes }}{\mathrm{cm}^{2}}$ at the center. While Equation (6) predicts a nearly linear rise in shear stress between 60 and 210 RPM, the shear stress the authors measured using optical Doppler velocimetry on the center and periphery of the dish was well below that of Equation (6) at all points except 210 RPM on the periphery, showing an exponential trend for both regions. The cause of the discrepancy is likely due to the fact that the equation represents shear stress at the center of the dish, while the authors took an average of values collected from the region.

\section{Use of Computational Fluid Dynamics to Quantify Shear Stress}

As analytically calculating wall shear stress can be complicated, researches have used computational fluid dynamics (CFD) software to better quantify the results. CFD has been used since the early 1930's with the advent of the Atanasoff automatic digital computer (Anderson et al, 1984). The role of computerized flow modeling with CFD software in vasculature applications is rapidly increasing due to the advancement of high 
performance computers in addition to more powerful commercially available software. CFD software is used for simulating, analyzing, and visualizing any type of fluid flow. This provides a means to determine fluid properties that are difficult or impossible to determine in vivo.

Early attempts using CFD software to solve the Navier-Stokes equations dealt with steady flow problems as applied to flow over a surface of cells. Sakurai et al. (1991) used CFD to study flow over cultured endothelial cells. To create the model, the authors used high definition optics at a 60x zoom to capture images of bovine arterial endothelial cells. Their results showed that shear stress at the highest point of the cell surface was 35 times greater than that on the substrate surface. Yamaguchi et al. (1993) studied shear stress distribution over confluently cultured endothelial cells. They used CFD to study models with varying cell structure. They found that the shear stress values varied greatly with the cell configuration. Both Sakurai et al. (1991) and Yamaguchi et al. (1993) assumed flow to have a linear velocity profile in their simulations. Using a wavy surface representing endothelial cells, Satcher et al. (1992) assumed local, linear shear flow in modeling the arterial endothelium.

Other CFD studies incorporate pulsatile flow into simulations and, in a number of cases, compare the CDF predictions with experimental data obtained from physical models. Weston et al. (1998) compared both pulsatile and steady flow through a $180^{\circ}$ pipe bend to the same flow conditions in CFD simulations through the pipe system. An MRI technique measured velocity in the actual pipe. The authors stated the CFD simulations for both types of flow showed similar results to the velocity images taken inside the pipe. Wall shear stresses could not be measured directly with the MRI 
technique, but instead were drawn from the CFD simulations. Banerjee et al. (2000) used CFD to evaluate hemodynamic effects as a result of a coronary artery balloon angioplasty. They noted the complexity of the flow field in the oscillatory region of the distal vessel. Papathanasopoulou et al. (2003) modeled pulsatile flow in a physiologically realistic model of the human carotid bifurcation. They found good agreement between MRI techniques and CFD simulations when comparing velocity measurements. Nonetheless, MRI techniques were not able to detect a region of high wall shear stress values in the disturbed flow along a divider wall that were forecasted by the CFD software. This finding led the authors to state that the addition of CFD modeling was highly advantageous in the study. Marshall et al. (2004) studied pulsatile flow in models of stenosed human carotid bifurcations with physical MRI models and CFD models. They also concluded that there is reasonable agreement between velocity measurements obtained from both media.

Although CFD software has advanced computational capabilities, no studies have modeled oscillatory fluid motion in an orbiting shaker, one of the most common in vitro cell culture apparatuses. Knowledge of the fluid dynamics inside an orbiting cylinder, covering a comprehensive set of operating conditions, would greatly enhance the value of orbital shaker apparatuses in the study of hemodynamic effects on endothelial cell cultures. 


\section{B. Dimensionless Parameters for Fluid Dynamics Analysis}

\section{Development of Dimensionless Parameters}

To analyze how shear stress develops in an orbital shaker apparatus, seven dimensional independent parameters would be required: $a$ is the cylinder radius, $h$ is the static height of the fluid in the cylinder, $\mathrm{R}$ is the radius of orbit, $\omega$ is the angular velocity of orbit, $g$ is the acceleration of gravity, $\mu$ is the fluid viscosity, and $\rho$ is the fluid density. A dish labeled with these seven parameters is seen in Figure 1. If cases were set up to view the effects of a change in each parameter at a low, medium, and high value, the total number of cases to view each combination of parameters would be 840 . It would take an unreasonable computational effort to study this number of cases.

The challenge is to reduce the number of variables to decrease computational effort. Rance and Warren (1968) demonstrated that a dimensional analysis can reduce seven independent variables to four dimensionless parameters. You (1997) used this principle in studying sheet flow in oscillatory flow. Using experimental data, he reduced a model of oscillatory flow in a sediment bed containing seven variables to a dimensionless equation containing only four variables. By taking one of the variables as constant the dimensionless model is reduced to three independent dimensionless parameters. 


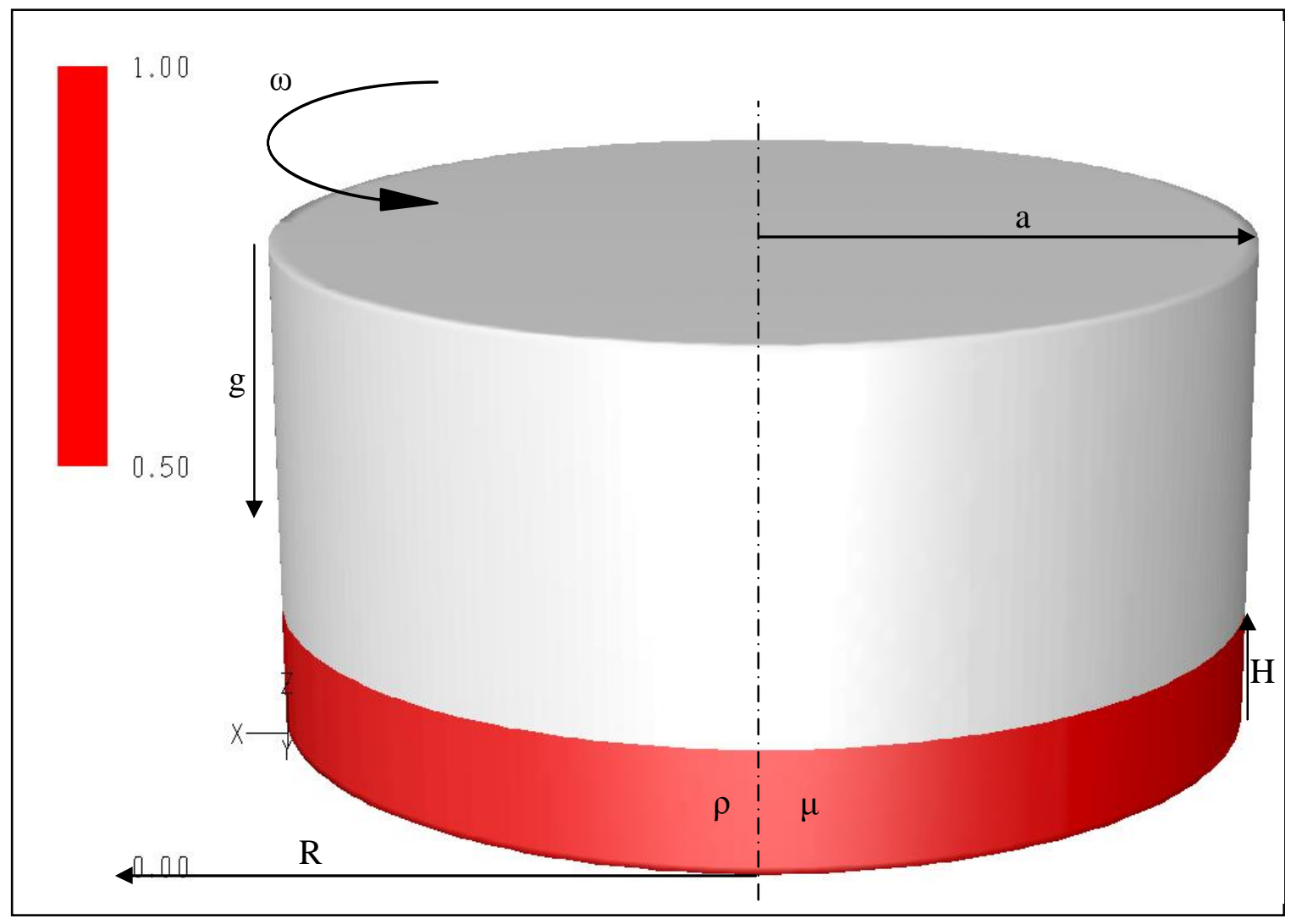

FIGURE 1 - Dish with Seven Parameters Labeled

The system of study here can also be reduced to three dimensionless parameters. A dimensional analysis of the seven parameters yields four dimensionless parameters: the Reynolds Number, the Stokes Number, The Froude Number and the Slope Ratio. Flow in the orbital shaker apparatus is limited here to laminar flow. Therefore, a constant laminar Reynolds Number is used to reduce the number of parameters that affect how shear stress develops in an orbital shaker apparatus to three. With three independent dimensionless parameters, a detailed study can be created with only 27 cases.

\section{Stokes Number}

Irish mathematician George Gabriel Stokes defined the Stokes Number. Stokes was a prolific force in the scientific community who co-developed the Navier-Stokes equations, one of the foundations of fluid dynamics. Starting in 1842, he began an 
interest in the field of hydrodynamics by studying motion of incompressible fluids (Wilson, 1987). In 1845 Stokes began investigating friction on fluids in motion. He furthered his research in 1850 to examine the effects of the internal friction of fluids on the motion. His findings explained many natural phenomena such as the suspension of clouds in air, and the subsidence of ripples and waves in water. As part of his studies on hydrodynamics, he calculated the terminal velocity for a sphere falling in a viscous medium. This calculation became known as the Stokes Law, which defined the frictional force exerted on spherical objects in a viscous fluid. Stokes examination of spheres in fluid also led to the development of the dimensionless Stokes Number. Hinds (1998) states that the most common use of the Stokes Number is in the aerosol industry where it is defined as the ratio of the stopping distance of a particle to a characteristic dimension of the obstacle, or:

$$
S t=\frac{\tau u}{d_{c}}
$$

For the system at hand, the Stokes Number has a modified definition where it is defined as the ratio of inertial forces from the dish versus viscous forces from the fluid, or:

$$
S t=H \sqrt{\frac{\omega}{v}}
$$


In determining the Stokes transition, it is desired to understand the effects of low and high Stokes Number on flow. For a low Stokes Number, the fluid motion will be viscous dominated and the fluid wave will oscillate in phase with the cylinder motion. For high Stokes Number, the flow will be inertially dampened, and the phase of the wave will lag behind the cylinder motion.

\section{Froude Number}

The Froude number was originally defined by English engineer William Froude in his Law of Comparison in 1868 (Brown and Lambert, 2004). Froude began studying the stability of ships in a seaway in 1861 . He used a sequence of 3,6 , and 12 foot scale models to measure the resistance each model offered when towed at a given speed. The Froude Number is the quantification of the resistance of floating objects and was developed so the results of small-scale tests could be used to predict the behavior of fullsized hulls.

The Froude Number compares convective and gravitational forces and is defined as a ratio of speed/length or:

$$
F r=\frac{u}{\sqrt{g H}}
$$

For the system at hand, the Froude Number has a somewhat modified definition where it is defined as:

$$
F r=\frac{a^{2} \omega^{2}}{g H}
$$


In an orbiting dish, the magnitude of the Froude Number determines the steepness of the leading edge of the wave. For an extremely low Froude Number, the leading edge has the same slope as the rest of the wave. As the Froude Number increases toward the transition, the slope of the leading edge becomes greater until a vertical wall is achieved. For high Froude Numbers, the wave front becomes unsteady and develops into a overshooting wave similar in appearance to an ocean wave as it crashes on a beach and may eventually crash.

\section{Slope Ratio}

Slope is used to measure the steepness of a straight line, with a larger slope value signifying a deeper slope. In its most basic form, slope is defined as rise over run or (Finney, 2001):

$$
m=\frac{\Delta Y}{\Delta X}
$$

For the system of study, the Slope Ratio is defined as the ratio of the quasi-steady free surface slope and the aspect ratio of the fluid at rest or:

$$
S l=\frac{R a \omega^{2}}{g H}
$$

The Slope Ratio affects the slope of the wave, with a greater slope resulting in a steeper wave relative to the dish aspect ratio. When a large Slope Ratio is achieved, the slope of the trailing edge of the wave will become so great that part of the base of the cylinder will 
become dry. To counteract the drying, viscous forces cause the trailing edge to extend to cover as much dry area as possible. The wave will no longer be uniform as the trailing edge of the wave becomes concave. The fluid is considered to have a high Slope Ratio when concavity is present for high Stokes Numbers. 


\section{EXPERIMENTAL}

\section{A. Experimental Plan}

To comprehensively define the effects of varying the three dimensionless parameters relative to each other, each of the 27 cases, represented by the black dots in Figure 2, would need to be investigated. The scope of this study, however, only includes the seven dots connected by the red lines in Figure 2.

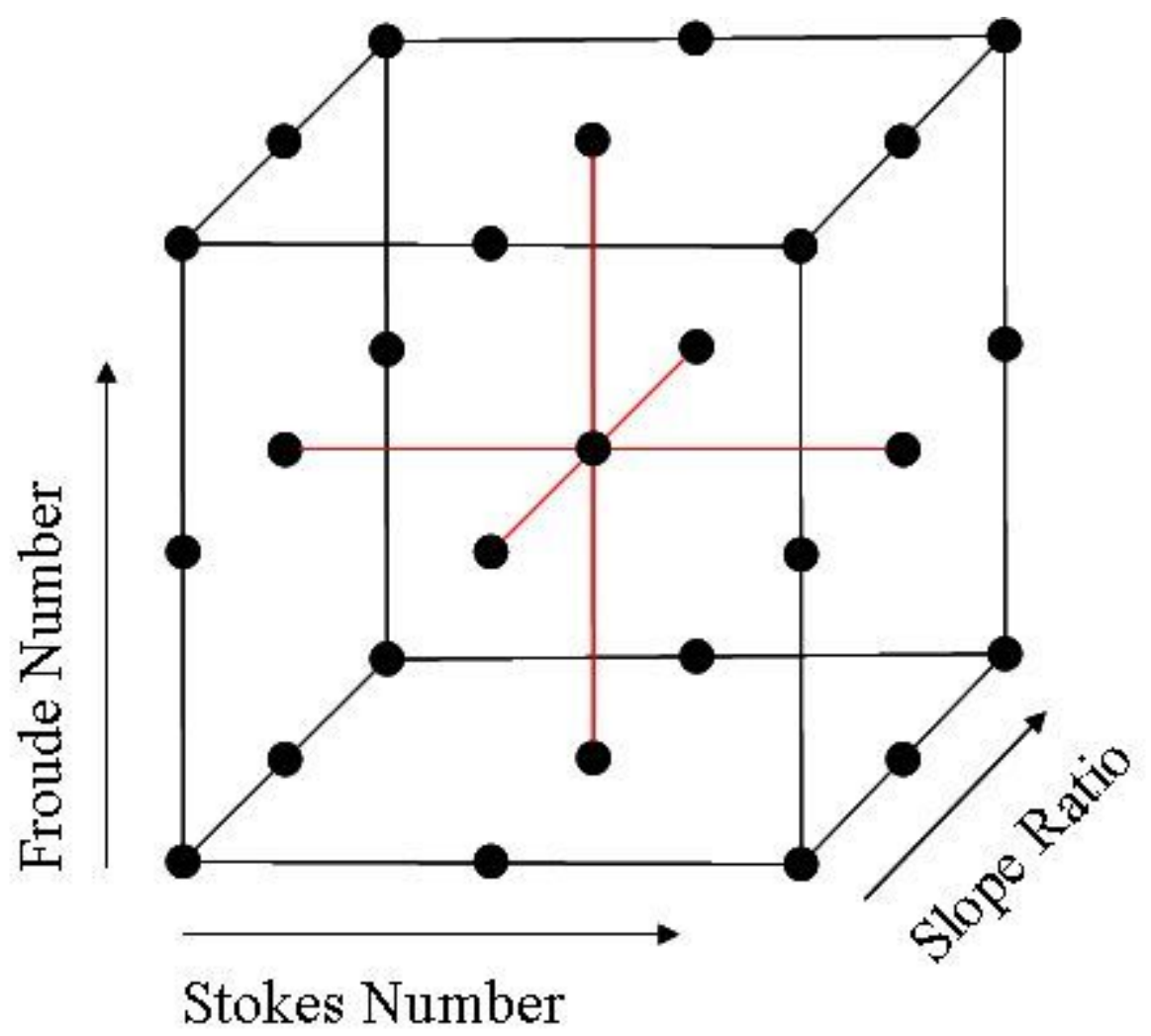

FIGURE 2 - Cube of Dimensionless Parameters

For this investigation, each of the three dimensionless parameters is varied while keeping the other two parameters near the expected transition value. It is unknown precisely where each of the transitions will occur in relation to the effects of the other two parameters, so several cases for the parameter of study will be examined between a 
definitively low value to a definitively high value. Additionally, having a greater number of cases allows investigation of shear values and other phenomena throughout the range of the dimensionless parameter.

The dimensionless parameters are examined as follows: The Slope Ratio is varied from 0.1 to 10 , the Froude Number is varied from 0.1 to 1.2 , and the Stokes Number is varied from 1.0 to 10. The middle values of the cube in Figure 2 were chosen as they were predicted to be near each dimensionless parameter's transition from high to low. By examining the middle cross of the cube in Figure 2, the two fixed dimensionless parameters are likely to have an effect on the characteristic of the wave. The middle values are: Slope Ratio of 1.0, Froude Number of 1.0, and Stokes Number of 5.0.

With the dimensionless parameter values defined for this study, Equations (9), (11), and (13) combined with a constant Reynolds Number of 100 can be modified to give values for the seven dimensional parameters needed to create and develop a CFD simulation. Radius of cylinder, mean height of fluid, orbital speed, and kinematic viscosity vary with the dimensionless parameters, while gravity, radius of orbit, and fluid density are held constant. Some of the orbital systems that develop from a determined set of dimensionless parameters yield cases that are experimentally improbable as dish sizes and orbital speeds are out of the range in which endothelial cell experimentation would occur. However, these cases are still valuable in determining overall shear trends in varying the dimensionless parameter.

Once initial results of each dimensionless parameter were determined, an extra set of cases was developed to give more definition in a region of interest, either to more 
accurately define the transition, or to study an effect that was observed. The final set of parameters studied, including the extra cases, are show in Tables I - III.

TABLE I

CASES FOR VARYING STOKES NUMBER

\begin{tabular}{|c|c|c|c|c|}
\hline $\begin{array}{c}\text { Stokes } \\
\text { number }\end{array}$ & $\begin{array}{c}\text { radius of } \\
\text { cylinder, } \\
\mathrm{cm}\end{array}$ & $\begin{array}{c}\text { mean } \\
\text { height of } \\
\text { fluid, cm }\end{array}$ & $\begin{array}{c}\text { orbital } \\
\text { speed, } \\
\mathrm{rad} / \mathrm{s}\end{array}$ & $\begin{array}{c}\text { kinematic } \\
\text { viscosity, } \\
\mathrm{m}^{\wedge} 2 / \mathrm{s}\end{array}$ \\
\hline 1 & 1.2000 & 0.0120 & 2.8577 & $4.12 \mathrm{E}-08$ \\
\hline 2 & 1.2000 & 0.0480 & 5.7155 & $3.29 \mathrm{E}-07$ \\
\hline 3 & 1.2000 & 0.1080 & 8.5732 & $1.11 \mathrm{E}-06$ \\
\hline 4 & 1.2000 & 0.1920 & 11.4310 & $2.63 \mathrm{E}-06$ \\
\hline 4.2 & 1.2000 & 0.2117 & 12.0025 & $3.05 \mathrm{E}-06$ \\
\hline 4.4 & 1.2000 & 0.2323 & 12.5740 & $3.51 \mathrm{E}-06$ \\
\hline 4.6 & 1.2000 & 0.2539 & 13.1456 & $4.01 \mathrm{E}-06$ \\
\hline 4.8 & 1.2000 & 0.2765 & 13.7171 & $4.55 \mathrm{E}-06$ \\
\hline 5 & 1.2000 & 0.3000 & 14.2887 & $5.14 \mathrm{E}-06$ \\
\hline 10 & 1.2000 & 1.2000 & 28.5774 & $4.12 \mathrm{E}-05$ \\
\hline
\end{tabular}

TABLE II

CASES FOR VARYING FROUDE NUMBER

\begin{tabular}{|c|c|c|c|c|}
\hline $\begin{array}{c}\text { Froude } \\
\text { number }\end{array}$ & $\begin{array}{c}\text { radius of } \\
\text { cylinder, } \\
\mathrm{cm}\end{array}$ & $\begin{array}{c}\text { mean } \\
\text { height of } \\
\text { fluid, cm }\end{array}$ & $\begin{array}{c}\text { orbital } \\
\text { speed, } \\
\mathrm{rad} / \mathrm{s}\end{array}$ & $\begin{array}{c}\text { kinematic } \\
\text { viscosity, } \\
\mathrm{m}^{\wedge} 2 / \mathrm{s}\end{array}$ \\
\hline 0.1 & 0.1200 & 0.0300 & 14.2887 & $5.14 \mathrm{E}-08$ \\
\hline 0.12 & 0.1440 & 0.0360 & 14.2887 & $7.41 \mathrm{E}-08$ \\
\hline 0.14 & 0.1680 & 0.0420 & 14.2887 & $1.01 \mathrm{E}-07$ \\
\hline 0.16 & 0.1920 & 0.0480 & 14.2887 & $1.32 \mathrm{E}-07$ \\
\hline 0.18 & 0.2160 & 0.0540 & 14.2887 & $1.67 \mathrm{E}-07$ \\
\hline 0.2 & 0.2400 & 0.0600 & 14.2887 & $2.06 \mathrm{E}-07$ \\
\hline 0.3 & 0.3600 & 0.0900 & 14.2887 & $4.63 \mathrm{E}-07$ \\
\hline 0.4 & 0.4800 & 0.1200 & 14.2887 & $8.23 \mathrm{E}-07$ \\
\hline 0.5 & 0.6000 & 0.1500 & 14.2887 & $1.29 \mathrm{E}-06$ \\
\hline 0.7 & 0.8400 & 0.2100 & 14.2887 & $2.52 \mathrm{E}-06$ \\
\hline 1 & 1.2000 & 0.3000 & 14.2887 & $5.14 \mathrm{E}-06$ \\
\hline 1.2 & 1.4400 & 0.3600 & 14.2887 & $7.41 \mathrm{E}-06$ \\
\hline
\end{tabular}


TABLE III

CASES FOR VARYING SLOPE RATIO

\begin{tabular}{|c|c|c|c|c|}
\hline slope ratio & $\begin{array}{c}\text { radius of } \\
\text { cylinder, } \\
\mathrm{cm}\end{array}$ & $\begin{array}{c}\text { mean } \\
\text { height of } \\
\text { fluid, cm }\end{array}$ & $\begin{array}{c}\text { orbital } \\
\text { speed, } \\
\text { rad/s }\end{array}$ & $\begin{array}{c}\text { kinematic } \\
\text { viscosity, } \\
\mathrm{m}^{\wedge} 2 / \mathrm{s}\end{array}$ \\
\hline 0.1 & 12.0000 & 3.0000 & 4.5185 & $1.63 \mathrm{E}-04$ \\
\hline 0.2 & 6.0000 & 1.5000 & 6.3901 & $5.75 \mathrm{E}-05$ \\
\hline 0.5 & 2.4000 & 0.6000 & 10.1036 & $1.45 \mathrm{E}-05$ \\
\hline 0.8 & 1.5000 & 0.3750 & 12.7802 & $7.19 \mathrm{E}-06$ \\
\hline 1.1 & 1.0909 & 0.2727 & 14.9861 & $4.46 \mathrm{E}-06$ \\
\hline 1.4 & 0.8571 & 0.2143 & 16.9066 & $3.11 \mathrm{E}-06$ \\
\hline 1.7 & 0.7059 & 0.1765 & 18.6302 & $2.32 \mathrm{E}-06$ \\
\hline 2 & 0.6000 & 0.1500 & 20.2073 & $1.82 \mathrm{E}-06$ \\
\hline 5 & 0.2400 & 0.0600 & 31.9505 & $4.60 \mathrm{E}-07$ \\
\hline 10 & 0.1200 & 0.0300 & 45.1848 & $1.63 \mathrm{E}-07$ \\
\hline
\end{tabular}

\section{B. Equipment and Software}

\section{Adelie}

Simulations are performed using Speed School of Engineering's Adelie Computer network, a 64 bit Linux cluster parallel system based on the AMD Opteron processor. The system consists of 6 nodes with a total of 12 processors yielding 43 Gigaflops of aggregate processor speed. Adelie contains 24 GigaBytes of memory, and 2.2 TeraBytes of disk storage. The operating system for Adelie is SUSE LINUX 9.1 Professional Edition.

\section{FLUENT Flow Modeling Software}

FLUENT version 6.2.5 is a commercial CFD software package that models fluid flow for any geometry. FLUENT is written in the C computer language but uses an interactive, menu driven interface for user access to the functions required to generate a solution and display results. The FLUENT solver is capable of modeling: 2D or 3D, steady state or transient, laminar or turbulent, incompressible or compressible, and 
Newtonian or non-Newtonian flow. Additional models are available for handling moving zones, species transport, heat transfer, acoustics, and chemical reactions. The solver employs a finite-volume discretization process to numerically solve the governing equations for conservation of mass and momentum. Equations for energy, turbulence, and species transport are solved when appropriate.

Computational models solve the complex Navier-Stokes equations that govern the system of interest. There are several basic equation that are used by a popular CFD modeler, FLUENT Flow Modeling Software, for laminar flow (FLUENT INC., 2001). The equations are as follows. For conservation of mass:

$$
\frac{\partial \rho}{\partial t}+\frac{\partial}{\partial x_{i}}\left(\rho u_{i}\right)=S_{m}
$$

For Conservation of momentum:

$$
\frac{\partial}{\partial x_{i}}\left(\rho u_{i}\right)+\frac{\partial}{\partial x_{j}}\left(\rho u_{j}\right)=-\frac{\partial p}{\partial x_{i}}+\frac{\partial \tau_{i j}}{\partial x_{j}}+\rho g_{i}+F_{i}
$$

Where the shear stress $\tau_{\mathrm{ij}}$ is:

$$
\tau_{i j}=\left[\mu\left(\frac{\partial u_{i}}{\partial x_{j}}+\frac{\partial u_{j}}{\partial x_{i}}\right)\right]-\frac{2}{3} \mu \frac{\partial u_{i}}{\partial x_{i}} \delta_{i j}
$$


After integration over the computational cells, the equations reduce to finite difference algebraic equations (FLUENT INC., 2001). The algebraic equations can be written in the common form:

$$
\phi_{p} \sum_{i}\left(A_{i}-S_{p}\right)=\sum_{i} A_{i} \phi_{i}+S_{c}
$$

The summations occur over each of the neighboring finite difference cells. The $A_{i}$ term is a coefficient which contains contributions from diffusive and convective fluxes. $S_{p}$ and $\mathrm{S}_{\mathrm{c}}$ are components in the linear source term:

$$
S_{\phi}=S_{c}+S_{p} \phi_{p}
$$

A differentiating scheme utilizing the power law is utilized to calculate the derivatives of the flow variables and interpolate between grid points. The algebraic equations are solved using a semi-implicit iterative scheme. The scheme starts at a given set of initial conditions and converges to a correct solution after executing a given number of solutions and/or reaching a specified tolerance.

Each iteration of FLUENT software involves several steps (FLUENT INC., 2001). First, the $u, v$, and $w$, momentum equations are each solved using pressure to update the velocity field. Subsequently, the velocities calculated may not satisfy the continuity equation. As such, an equation resembling the Poisson is derived from the continuity equation and the momentum equations to correct for pressure. Solving this equation yields corrections for pressure and velocity fields that allow the conditions of 
the continuity equation to be satisfied. Then auxiliary equations are solved using the updated velocities from the previous step. Next, the fluid properties are updated with the new complete set of values. Finally, the solver checks the set of equations for convergence. The steps are repeated until either the residual difference between iterations reaches a user-defined value, or a defined number of iterations is reached.

Simulating flow in an orbiting cylinder requires creating a dynamic grid that moves through space. The motion is described in a user-defined function (UDF) by specifying the orbital frequency, orbital radius, and center of orbit. The UDF is read into FLUENT using the moving dynamic mesh (MDM) capability. The UDF is read into FLUENT as part of a MDM library. A transient solution is required since the location of the fluid domain is changing with time and the solution takes several rotations to achieve steady state.

The sloshing of the fluid as a result of the motion is a free surface flow that requires tracking of the liquid-air interface. The surface tracking is accomplished with the Volume of Fluid (VOF) model. In the VOF model, the two fluids across the interface share a single set of momentum equations and the volume fraction of each fluid in each computational cell is tracked throughout the grid.

\section{Gambit CFD Preprocessor}

GAMBIT version 2.3.16 is a commercial CFD preprocessing software developed for optimal integration with FLUENT. As a preprocessor for engineering analysis, GAMBIT serves two functions. The solid geometry modeler has tools that can create and merge basic geometric shapes. Models can be built directly within GAMBIT's solid geometry modeler, or imported from any major CAD/CAE system. With GAMBIT's 
meshing tools, geometry is developed into a computational grid that is divided into discrete control volumes. A two dimensional grid may consist of triangular or quadrilateral computational cells. A three dimensional grid may consist of tetrahedral, hexahedral, pyramid, wedge, or hybrid cells.

\section{Procedure}

\section{Create Dish in GAMBIT}

Open the GAMBIT preprocessing software. The Petri dish is modeled as a closed cylinder. The diameter of the cylinder is predetermined from dimensionless parameters used for the case. The height is arbitrarily set as the lowest value where fluid will not strike the top of the cylinder (note that too shallow of a dish will develop an unsolvable model because the fluid will hit the top and too tall a dish will increase computational time as computational effort is wasted on the air region where nothing important occurs). To create a cylinder in GAMBIT, open the Volume menu by left clicking the Volume Command Button in the Geometry menu. In the Volume menu, right click on the Create Volume button and select cylinder. Set Height and Radius 1 to predetermined values and click Apply; Radius 2 is ignored in the cylinder creation.

Once the cylinder is created, a mesh is applied to allow calculations to occur in FLUENT. Approximately 300,000 cells are used for good accuracy at a reasonable calculation time (note that the exact number of cells will vary slightly from case to case). The use of 300,000 cells is determined from FLUENT's recommendation that increasing the cell count until a further increase by five percent changes the results by less than or equal to 0.1 percent. The mesh is created in three steps to allow control of the cells based on height to diameter ratio. Mesh controls are accessed by left clicking the Mesh Command 
Button. First, a mesh is applied to the radial edge of the cylinder. The Mesh Edges menu is activated by left clicking the Edge Command Button in the Mesh menu. A Mesh Edges window will appear below the Edge menu. Select the base of the cylinder; edge.1 will show in the yellow Edge window. Under spacing, right click on the tab that displays Interval Size and select Interval Count. The exact Interval Count required is initially unknown and trial and error is utilized to find an Interval Count to yield approximately 300,000 cells. Input the Interval Count and then left click Apply to apply the edge mesh to the base.

Next, a mesh is applied across the curved outer surface of the cylinder. The Mesh Faces menu is triggered by left clicking the Face Command Button in the Mesh menu. Select face.2, the curved face on the dish. Input the desired projected intervals into the newly appearing Projected Intervals input box. The Projected Interval is selected from an algorithm based on cylinder height and diameter (the algorithm is located the Appendix). Left click Apply to apply the face mesh to the curved face. Finally, the complete volume mesh is created. The Mesh Volumes menu is activated by left clicking the Volume Command Button in the Mesh menu. Select the cylinder volume and click Apply to complete the volume mesh.

Examine the number of mesh volumes in the Transcript. If the value is not close to 300,000 , click the Undo button in the Global Control Menu until all layers of the mesh are removed and then repeat with an adjusted interval count on the base edge. Once an acceptable mesh is created, it is exported to a file type usable by FLUENT. The mesh is exported by clicking on File, Export, and then Mesh. Type a name in new Export Mesh File window and click Accept to export the mesh. GAMBIT may now be closed. 


\section{Import Dish into FLUENT and Set Parameters}

Open the 3D version of FLUENT software. To import the mesh created in GAMBIT, click File, Read, and then Case; a Select File window will open. Under Files, left click on the .msh file and then left click on OK. While the Petri dishes used here is measured in centimeters, Fluent defaults all length units to meters. Default units are changed by clicking on Grid and then Scale. In the Scale Grid window: click on the down arrow, select "cm", click on Change Length Units, click on Scale and click Close to close the window. The solver must be set to unsteady state to operate properly. Click Define, Models, and then Solver to open the Solver window. Under Time, click Unsteady and then click OK to close the window.

The cylinder contains both air and water so Fluent must be set to multiphase operation. Click Define, Models, and Multiphase to open the Multiphase Mod window. Under Model, click Volume of Fluid and then click OK (note that several forms will appear, but they may be ignored). The materials in the dish must be specified. Air is preloaded, but liquid water has to be added. Click Define and then Material to open the Materials Window. Click Fluid Database to open the Fluid Database Materials window. Scroll down under Fluent Fluid Materials and select water-liquid, click Copy and then click Close (note that 1-buten-3-yne-2yl-radical is initially selected; click to deselect). Click on Close to close the Materials window.

With materials selected, they need to be assigned to the respective phases. Click Define and then Phases to open the Phases window. For most efficient calculation, set the primary phase as air and the secondary phase as water. To set a phase, select the Phase and then click Set; a window for either the Primary or Secondary phase will 
appear. Select the desired material from the Phase Material drop down box and type its name in the Name box. Click OK to set the phase. When both phases are set, click Close.

Now that the phases are set, operating conditions in the cylinder may be set. Click Define and then Operating Conditions to open the Operating Conditions Window. Under Reference Pressure Location, type a value just less than the height of the dish in the $\mathrm{z}$ direction (for example if a dish is $6 \mathrm{~cm}$ tall, set at $5.9 \mathrm{~cm}$; set at top so reference pressure region is always occupied by air). Click Gravity in the Gravity menu to open the Gravitational Acceleration form. Input $-9.81 \frac{\mathrm{m}}{\mathrm{s}^{2}}$ for the z component then click OK.

Now that the operating conditions are set on the cylinder, motion can be added. Motion is created as a User Defined Function (UDF) in C++ code. After a UDF is created, it is loaded into Fluent. An example of the UDF used is in the Appendix; the only portion of the code which changes is the orbital speed $(\omega)$ in radians per second (note that in the code $w$ is used in place of $\omega$ ). To load the UDF, click Define, UserDefined, Functions, and then Compiled to open the Compiled UDF Window. Under Source Files, click Add to open the Select File window. Select the UDF file and click OK. Next to Library Name type mdmlib_followed by a descriptive term for the case and then click Build to construct the library e.g. mdmlib_slope.1. The mdm library must be in the same directory that FLUENT is running from or the motion will not be utilized during computation. After the library is built, click Load to load the library.

The motion can now be attached to the mesh. First, the Dynamic Mesh must be activated. This is accomplished by clicking Define, Dynamic Mesh, and then Parameters to open the Dynamic Mesh Parameters Window. Click Dynamic Mesh under Models and 
click OK. Now the motion will be attached to the fluid and to the cylinder. Click Define, Dynamic Mesh, and then Zones to activate the Dynamic Mesh Zones window. Under Zone Names select fluid, check that the motion is present under Motion UDF/Profile and click Create. Repeat for the wall zone and click Close.

The interior of the cylinder initially contains air throughout its interior. A separate region must be created to represent the fluid; the fluid is introduced as a volume patch. To patch a volume to a region, first the solver must be initialized. To initialize the solver, click Solve, Initialize, and then Initialize to open the Solution Initialization window. Click Init and then Close to initialize the solver (note for this particular case it is not required to select a region from the Compute From box). Now select the region to change to fluid by clicking Adapt and then Region to open the Region Adaptation window (Figure 3). Under Input Coordinates, on X Min and Y Min input the negative cylinder radius, and input the cylinder radius for X Max and Y Max. Z Min remains 0 and $\mathrm{Z}$ max is the predetermined mean height of the fluid. Click Mark and then close to mark this region. The new marked region can now be set as liquid. Click Solve, Initialize, and then Patch to open the Patch Window. Under Phase, click the down arrow and select water and then under Value type one. Under Registers to Patch select hexahedron-r0 then click Patch to patch the liquid to the base of the cylinder. 


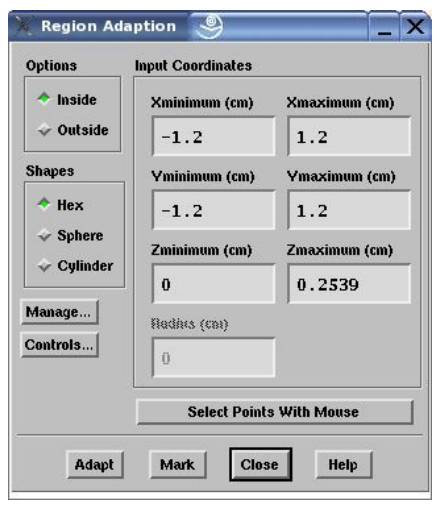

FIGURE 3 - Region Adaptation Menu

Calculation parameters may now be adjusted. The time step must be set to allow for accurate computation. To adjust the time step, open the Iterate window by clicking Solve then Iterate. To set the time step, type 0.001 after Time Step Size and click Apply and then Close. This value is chosen for the time step because convergence problems occur at larger increments. The methods the solver uses to calculate the solution can be adjusted in the Solution Controls menu. This menu is accessed by clicking on Solve, Controls and then Solution. The parameters set as follows: each of the Under-Relaxation factors are set at 0.3, the Pressure-Velocity Coupling is set as PISO, the Discretization for pressure is set as Body Force Weighted, the Discretization for momentum is set as QUICK. Click OK to close. The Solution Controls menu can be seen in Figure 4. Lastly, the convergence criteria are set. Click Solve, then Monitor, then Residual to open the Residual Monitors window. Set the Residual for Continuity and $\mathrm{x}, \mathrm{y}$, and $\mathrm{z}$ velocities all at 0.0001 . This value was determined by varying the residuals to find the quickest convergence without affecting the results. 


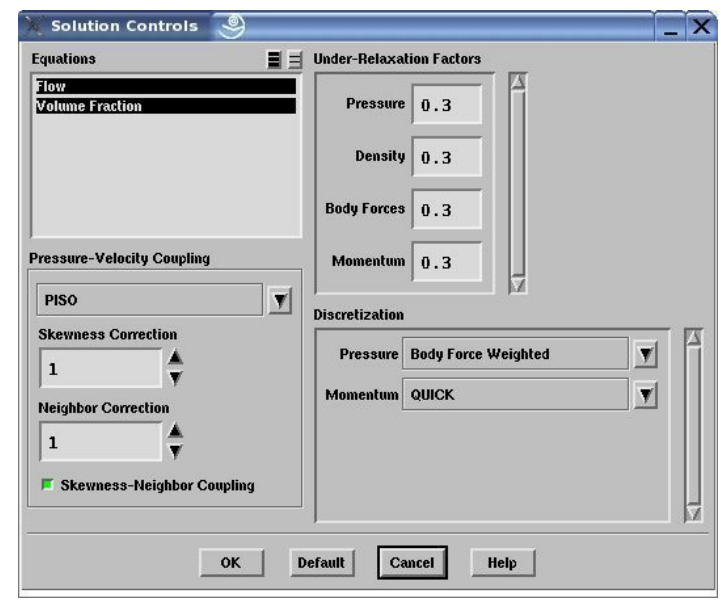

FIGURE 4 - Solution Controls Menu

Before saving, the auto save feature must be activated to allow investigation of the progress of a simulation while it is running. This feature tells FLUENT to save case and data files at predetermined intervals. The Autosave Case/Data window is open by clicking on File, Write, and then Autosave. Autosave Case File Frequency and Autosave Data File Frequency are equal, determined from the equation:

$$
A S F=785.4 \omega^{-1}
$$

Enter an arbitrary value under Maximum Number of Each File Type. A large number is desired so that all time steps are saved. Finally, the Case and Data File are saved. Click File, Write, then Case and Data to open the Select File menu. Type a descriptive name for the case and click OK to save. Close FLUENT.

\section{Set-Up Case to Run in Batch Mode}

To expedite convergence of case files, they are run in a batch mode on dedicated nodes on the Adelie supercomputer. Two files are needed to run a FLUENT case file in batch mode, a batch file and an input file. The batch file opens FLUENT and initializes it 
to run on a batch node. The sixth line of the batch file indicates the directory from where FLUENT reads the input file, and the seventh line dictates to which directory the output from FLUENT is read. Once FLUENT is initialized, operational commands are given in the input file. Lines one and two load the initial case and data file for the simulation. Line five sets the number of time steps, and line six sets the number of iterations per time step. To set the maximum number of iterations per time step for maximum convergence speed, it is usually advantageous to run a small number of time steps (five to ten) and then set the maximum roughly five iterations greater than the maximum observed. FLUENT bases the time allocation for a time step on the total number of iterations, therefore a large excess in iterations can slow down the process. Lines seven and eight record the final case and data files once the simulation is complete. An example of the batch and input file are located in the Appendix.

\section{Analyze Data}

Two criteria in each case are viewed after the simulation is complete. First, the shape of the wave is observed, and then the wall shear stress on the bottom of the dish is inspected. To view the cylinder, open the Contours menu by selecting View and then Contours. To view the shape of the wave, under Contours Of select Phases, under Levels select two, click Filled under Options, select Wall and click Display to open a contours window. This is seen in Figure 5. The dish can be rotated with the mouse until a desired view of the wall is established. To capture a picture, click File and then Hardcopy to open the Graphics Hardcopy Window. Click JPEG under Format and Color under Coloring and click Save. Name the file and a picture is created. Shear stress is viewed in a similar way. In the contours menu set Levels at 100. In the first box under Contours 
Of select Wall Fluxes, and in the box under that, select either Wall Shear Stress, X-Wall Shear Stress, or Y-Wall Shear Stress and click display. Wall shear stress is used for finding the maximum shear on the bottom of the dish, and $\mathrm{X}$ and $\mathrm{Y}$ wall shear stresses are used to create elliptical plots. To find the shear at a point, rotate the dish to the bottom and right click on the point to determine the shear for that area.

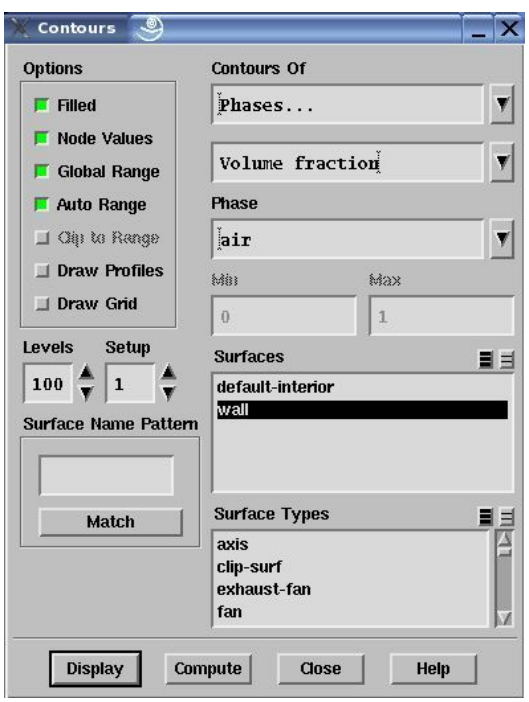

FIGURE 5 - Contours Window 


\section{RESULTS AND DISCUSSION OF RESULTS}

\section{A. Analysis of Free Surface Plots}

\section{Free Surface Plots as Function of Stokes Number}

In studying the free surface plots of the cylinder of study as the Stokes Number is varied from low to high values, the feature of paramount importance is the location of the wave with respect to the location of the dish. To determine the Stokes transition, each of the cases is considered at a point in time with the location of the dish in its orbit shown from the right side view of the free surface plots in FLUENT. Hence at the right side view, the peak of the wave will correlate to the center of the center point of the cylinder before the Stokes Transition is achieved. After the Stokes Transition, the peak of the wave will not reach the center point of the right side view.

To determine the degree of phase lag, the grid is enabled in FLUENT. The number of cells around the perimeter of the cylinder is known, therefore if $360^{\circ}$ is divided by the number of cells it gives the size of an individual cell. On a zoomed view of the peak of the wave with an active grid, the number of cells between the center of the right side view and the peak of the wave are counted. The number of cells counted between the center line and the peak of the wave multiplied by the size of an individual cell gives the degree of phase lag in the cylinder. An example of this procedure is seen in Figure 6. The yellow $\mathrm{X}$ denotes the reference location on the dish, and the green $\mathrm{X}$ denotes the location of the peak of the wave. 

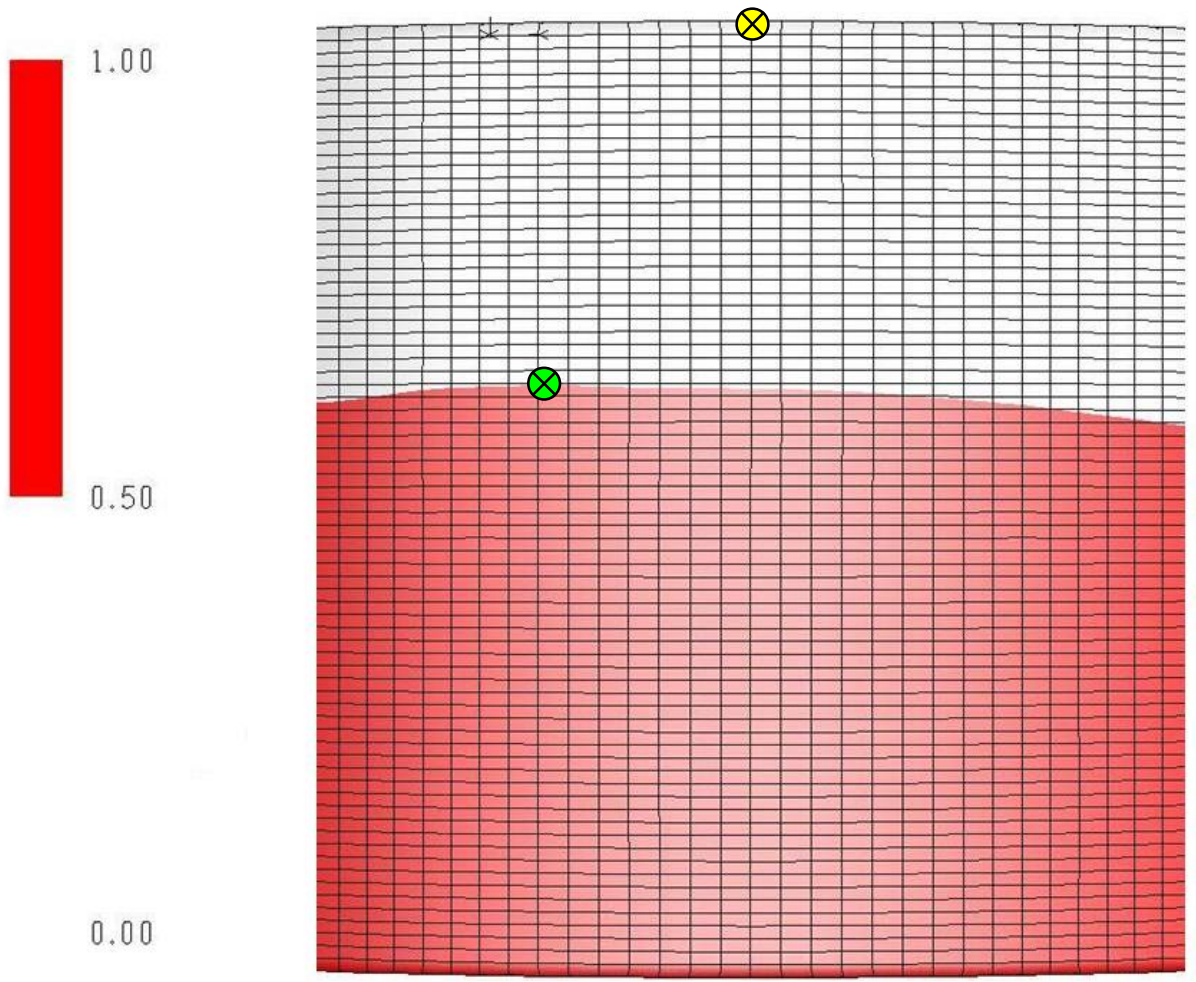

FIGURE 6 - Grid for Determining Phase Lag

The initial cases of study were for Stokes Numbers of 1.0 through 5.0 and 10 with a Froude Number of 1.0 and a Slope Ratio of 1.0. At a Stokes Number of 1.0, as seen in Figure 7, the peak of the wave meets precisely at the center point of the right side view indicating no lag is present. The same holds true for Stokes Numbers 2.0 through 4.0, as is seen in Figures 8 through 10. On the initial run of cases, a phase lag of $11.6^{\circ}$ is encountered at a Stokes Number of 5.0 as seen in Figure 11. Figure 12 shows a much larger phase lag of $317.8^{\circ}$ is present for a Stokes Number of 10. A black line is used to denote the centerline in all figures investigating Stokes effects. 


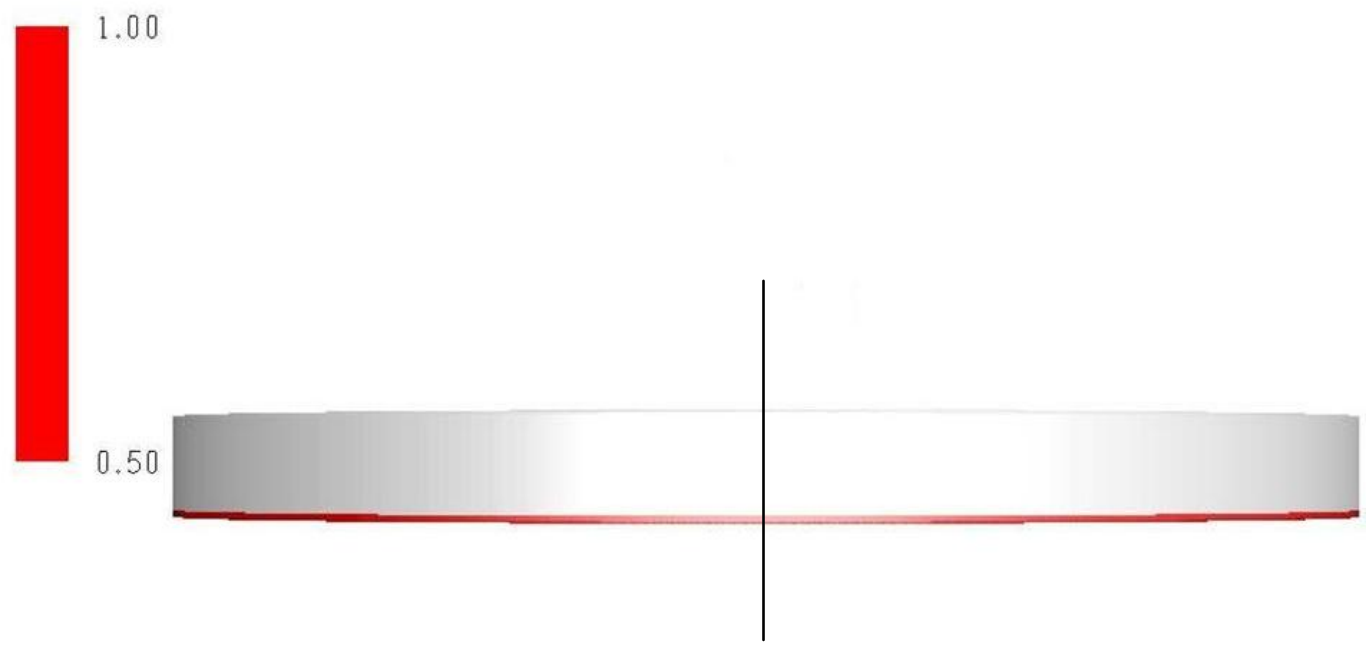

0.00

FIGURE 7 - Stokes Number 1.0 Free Surface Plot

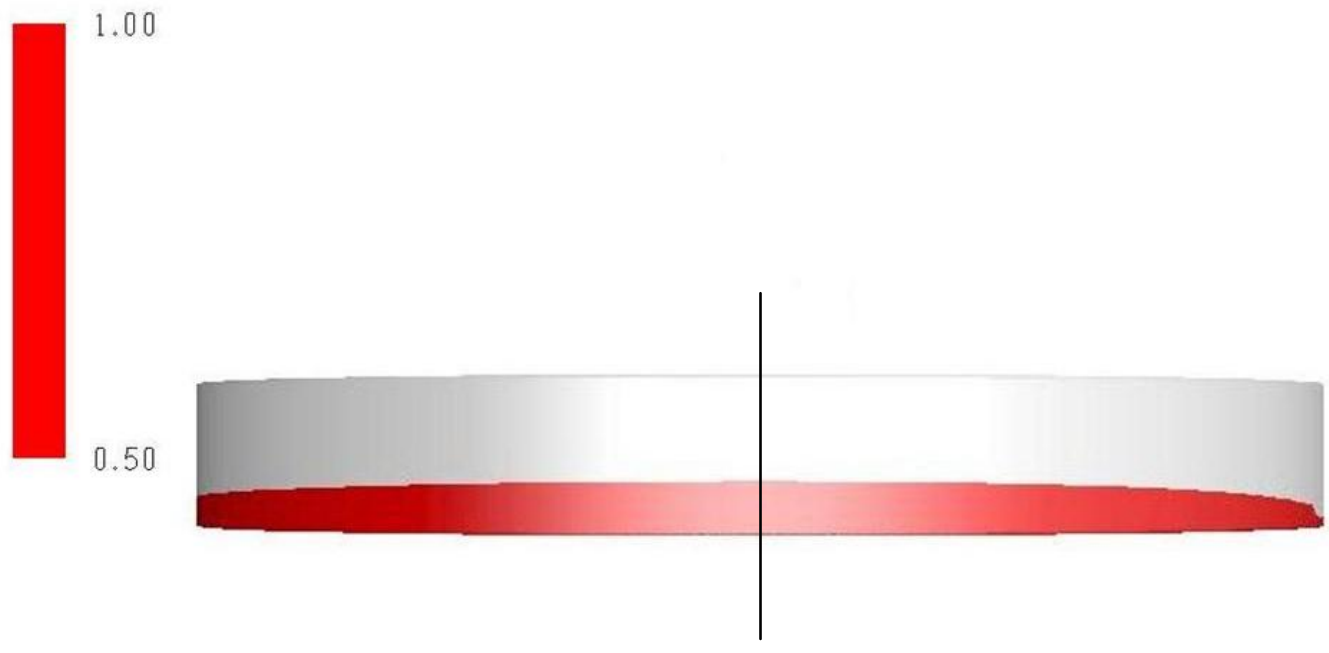

0.00

FIGURE 8 - Stokes Number 2.0 Free Surface Plot 


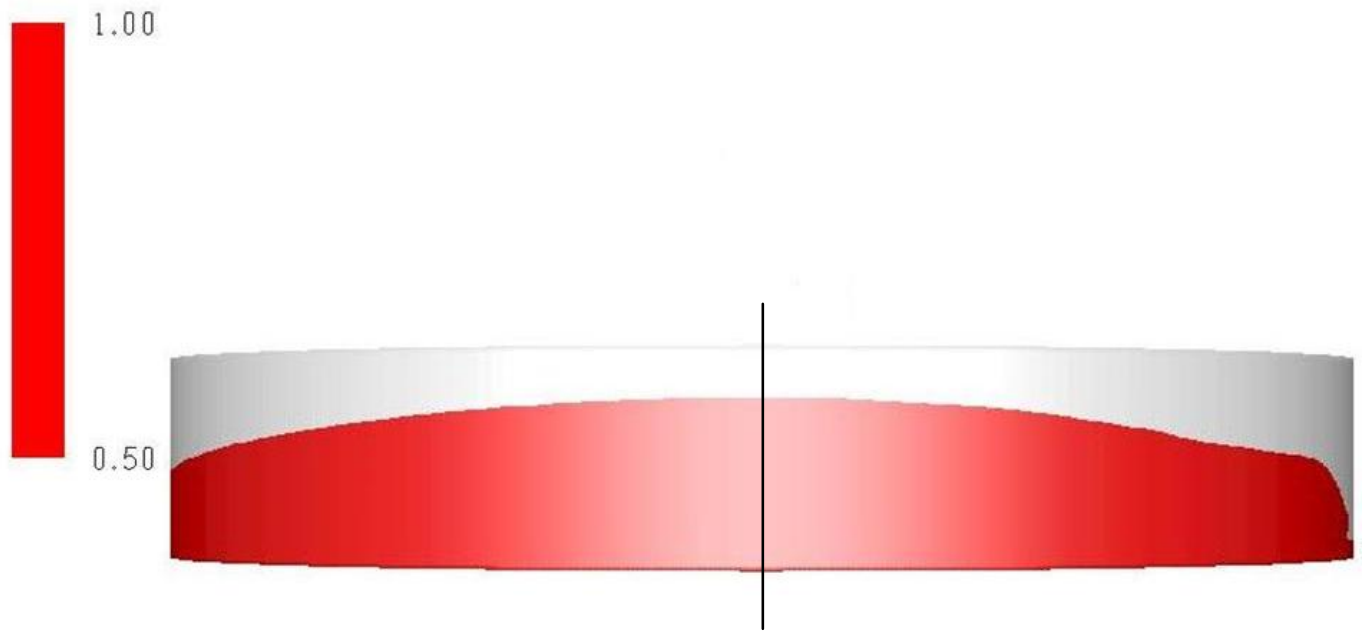

0.00

FIGURE 9 - Stokes Number 3.0 Free Surface Plot

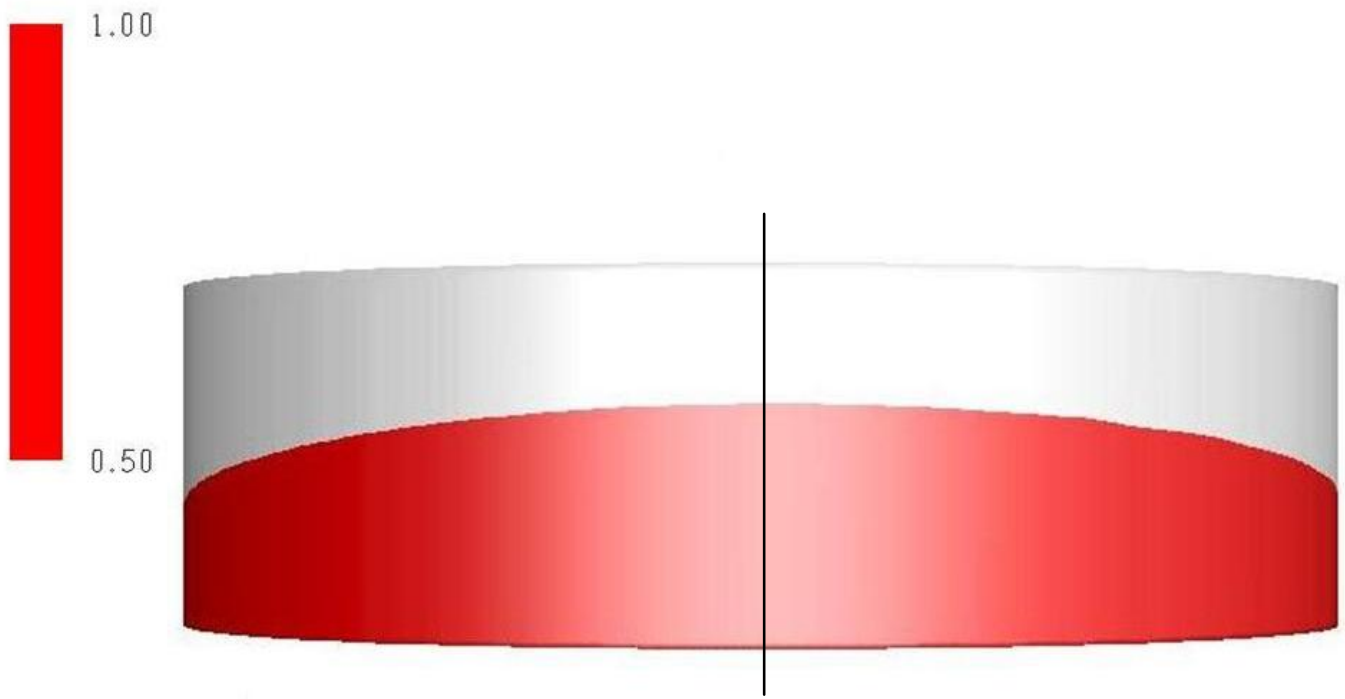

0.00

FIGURE 10 - Stokes Number 4.0 Free Surface Plot 


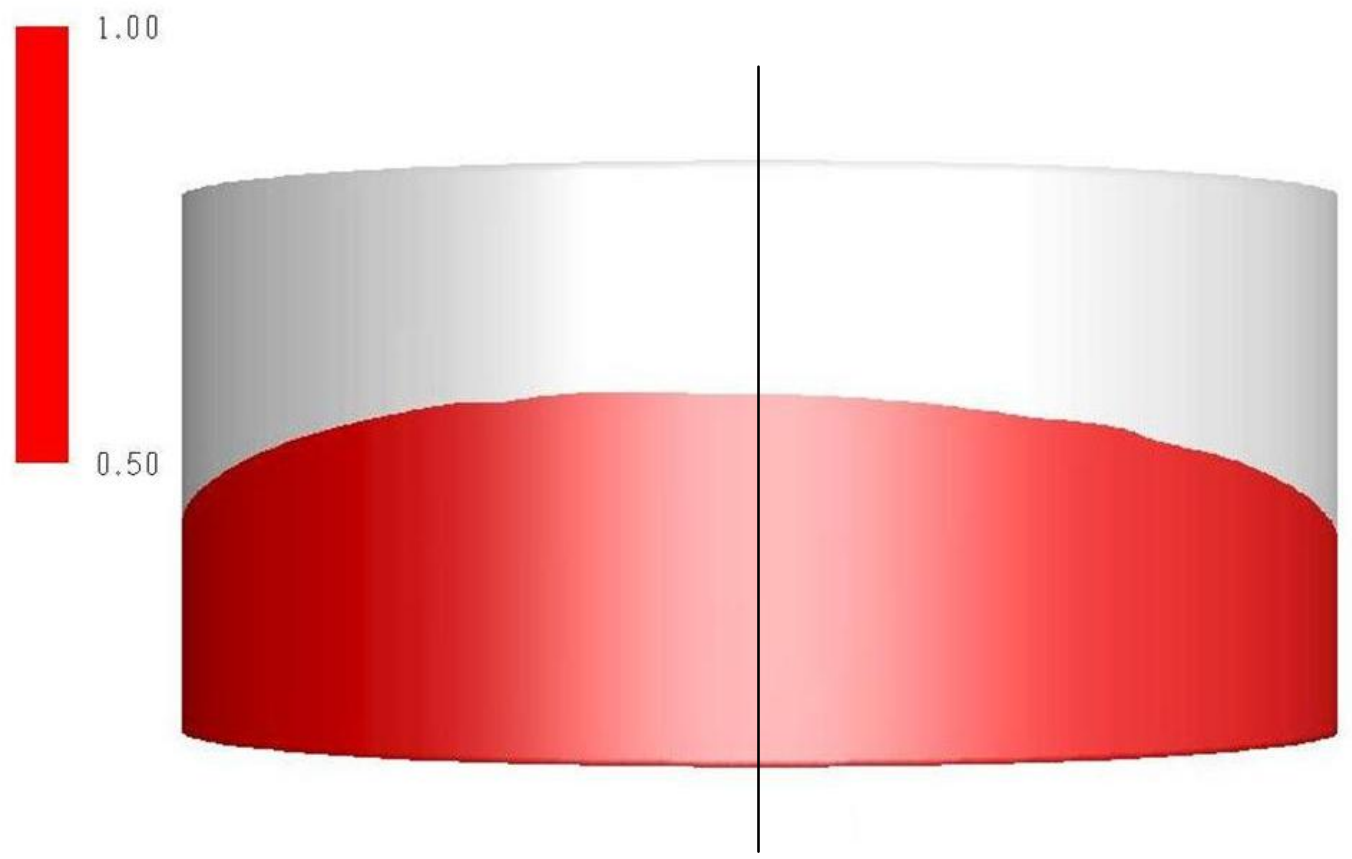

0.00

FIGURE 11 - Stokes Number 5.0 Free Surface Plot

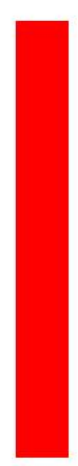

\subsection{0}

0.50

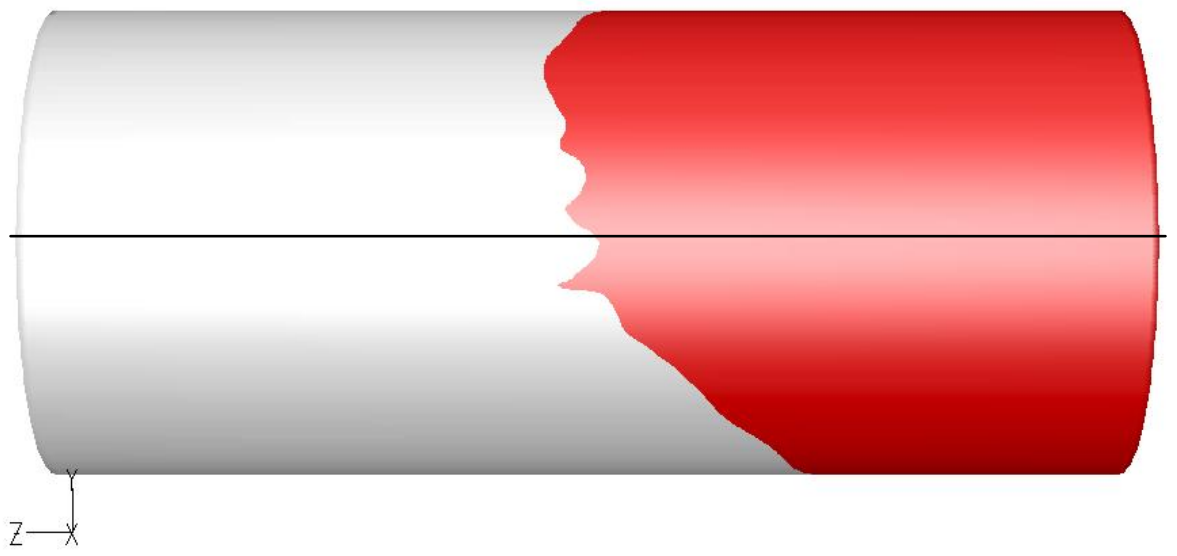

0.00

FIGURE 12 - Stokes Number 10 Free Surface Plot 
A phase lag of $11.6^{\circ}$ indicates that the transition occurs somewhere below a Stokes Number of 5.0 since the phase lag will increase as the Stokes Number is increased past the transition. Ideally, a minimal phase lag would be visible at the beginning of the Stokes transition. With a more precise location for the Stokes transition, its effects of the transition on shear can be more accurately documented. As such, a subsequent set of four equally spaced cases with Stokes Numbers of 4.2, 4.4, 4.6, and 4.8 are conceived to more accurately pinpoint the location of the Stokes transition.

When the results for the four additional cases are visually inspected, each shows a phase lag as is seen in Figures 13-16. Based on these findings, the Stokes transition for this set of dimensionless parameters occurs at a Stokes Number of $4.1 \pm 0.1$. Although the transition could be documented to several decimal places of accuracy, that degree of precision is not part of the scope of this study.

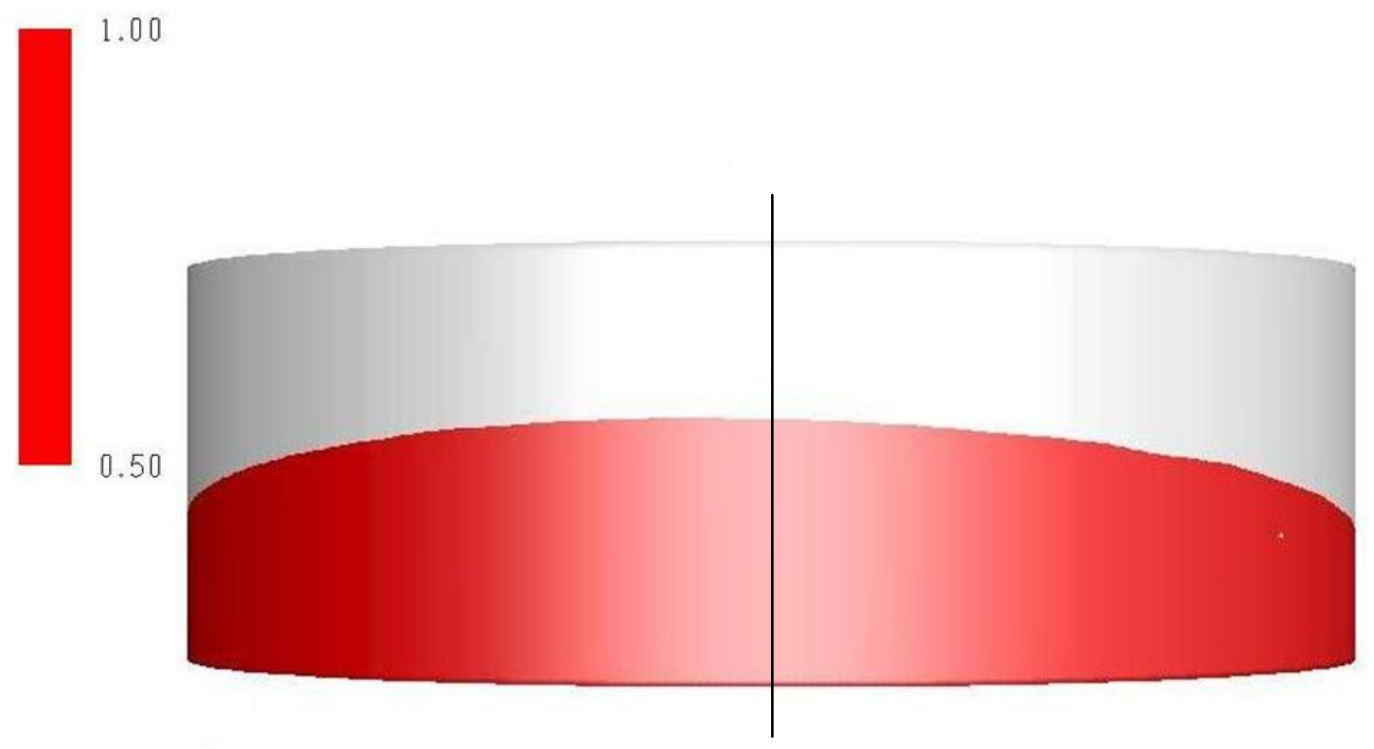

FIGURE 13 - Stokes Number 4.2 Free Surface Plot 


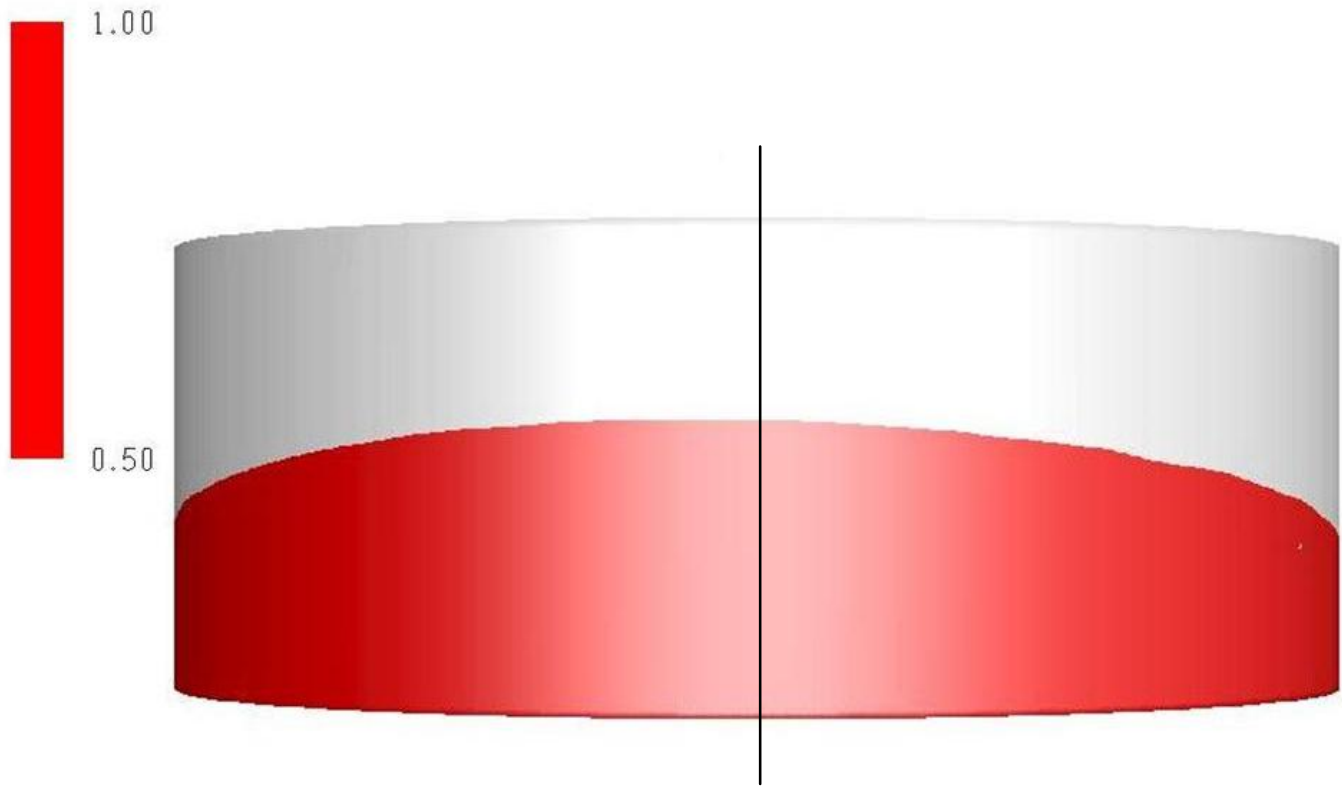

0.00

FIGURE 14 - Stokes Number 4.4 Free Surface Plot

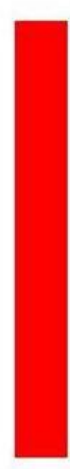

1.00

0.50

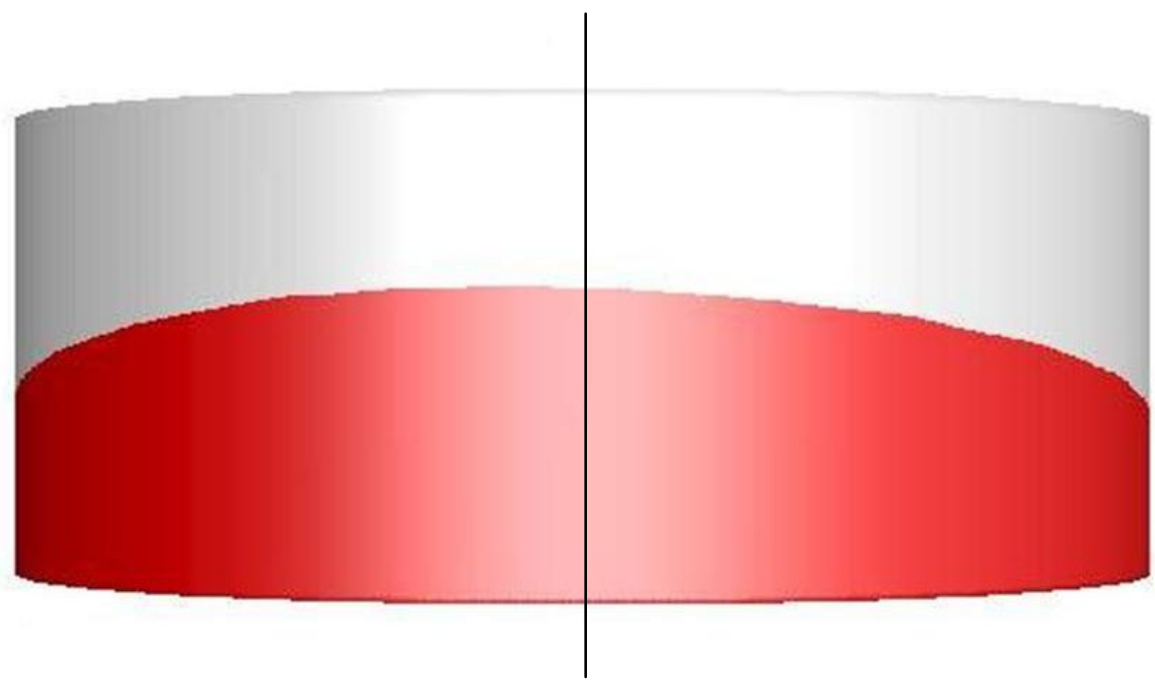

0.00

FIGURE 15 - Stokes Number 4.6 Free Surface Plot 


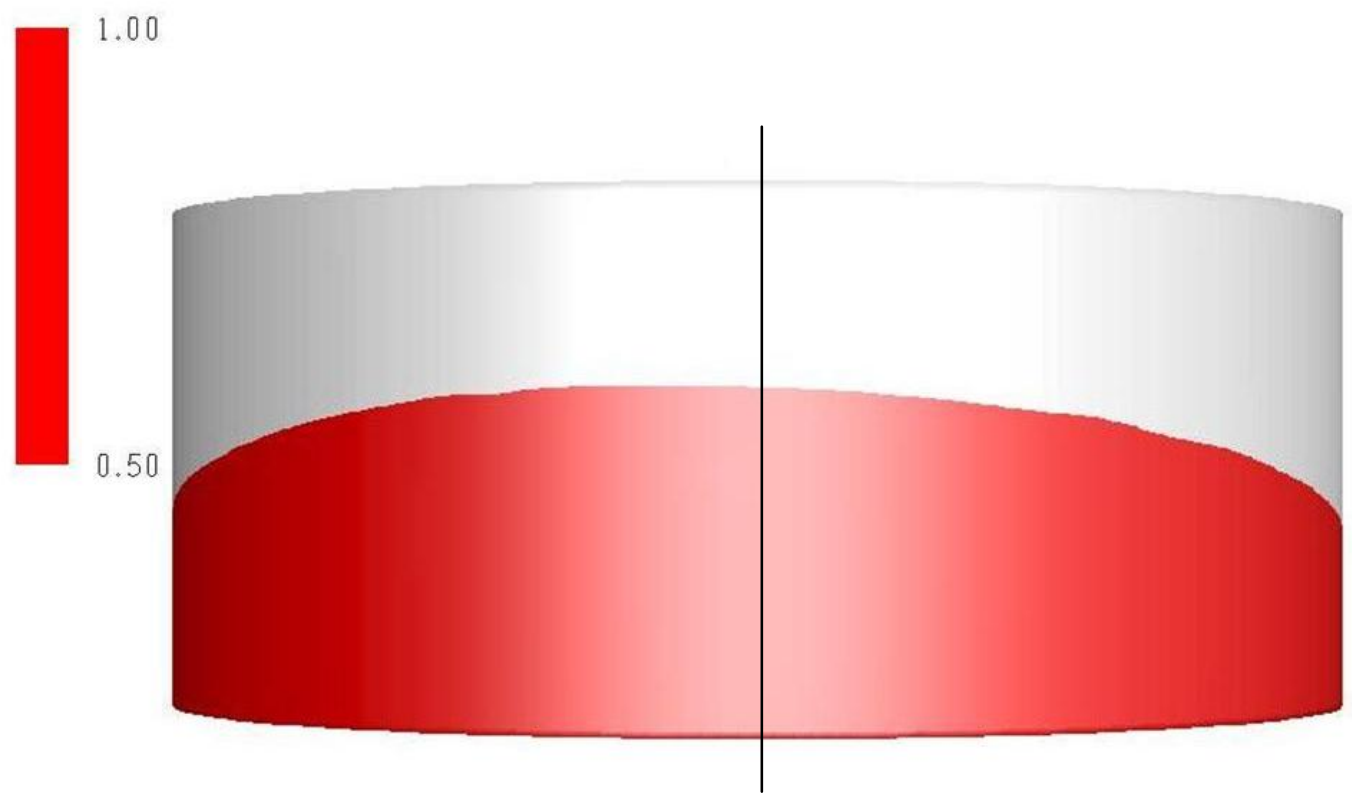

FIGURE 16 - Stokes Number 4.8 Free Surface Plot

Table IV show the value of phase lag for each of the Stokes cases. The data show that when the Stokes transition is crossed, a phase lag of several degrees quickly develops. For the subsequent nearby Stokes Numbers, the lag raises slightly as Stokes Number is increased. The case for the Stokes Number of 4.4 does not show this pattern though. The phase lag actually decreases instead of increases at this value. This is likely caused by some degree of computational error. When the Stokes Number shifts from 5.0 to 10 a huge increase is seen in the phase lag. Without further cases in the high range, it is impossible to tell how exactly such a large lag develops. It is unknown whether it suddenly becomes large or if it is a more gradual transition. 
TABLE IV

PHASE LAG OBSERVED VS. STOKES NUMBERS

\begin{tabular}{|c|c|}
\hline Stokes Number & Phase Lag \\
\hline 1.0 & $0.0^{\circ}$ \\
\hline 2.0 & $0.0^{\circ}$ \\
\hline 3.0 & $0.0^{\circ}$ \\
\hline 4.0 & $0.0^{\circ}$ \\
\hline 4.2 & $7.5^{\circ}$ \\
\hline 4.4 & $6.2^{\circ}$ \\
\hline 4.6 & $9.4^{\circ}$ \\
\hline 4.8 & $11.2^{\circ}$ \\
\hline 5.0 & $11.6^{\circ}$ \\
\hline 10.0 & $317.8^{\circ}$ \\
\hline
\end{tabular}

\section{Free Surface Plots as a Function of Froude Number}

As with the Stokes transition, the Froude transition is determined from visual inspection, although the leading edge of the wave is examined rather than the wave's peak. Although the Froude transition is the key element of study, observing the progression of the Froude effect as the values pass from low to high is also insightful in understanding the shear profiles that ensue.

The initial cases of study were for Froude numbers from 0.1 through 0.5, 0.7 and 1.0 for a Stokes Number of 5.0 and a Slope Ratio of 1.0. The Froude effect witnessed varies greatly from low to high so it will be explained in three segments: the transition value will be defined, free surface plots from the low value to the transition value will be explored, and then free surface plots from the transition value to the high value will be surveyed.

When observing free surface plots for the Froude Numbers equal to 0.2, and 0.3, as seen in Figures 17 and 18, the Froude transition is easily seen. At the Froude Number equal to 0.2 , the vertical wall effect is visible signifying this value is in the low Froude 
region. At the Froude Number of 0.3 , a rippled effect is seen on the leading edge of the wave. As described earlier, the Froude transition is the value where the vertical wave effect breaks up and gives way to a standing wave. Since the breakup of the wave occurs between 0.2 and 0.3 , the Froude transition is defined as occurring at a Froude Number of $0.25 \pm 0.05$.
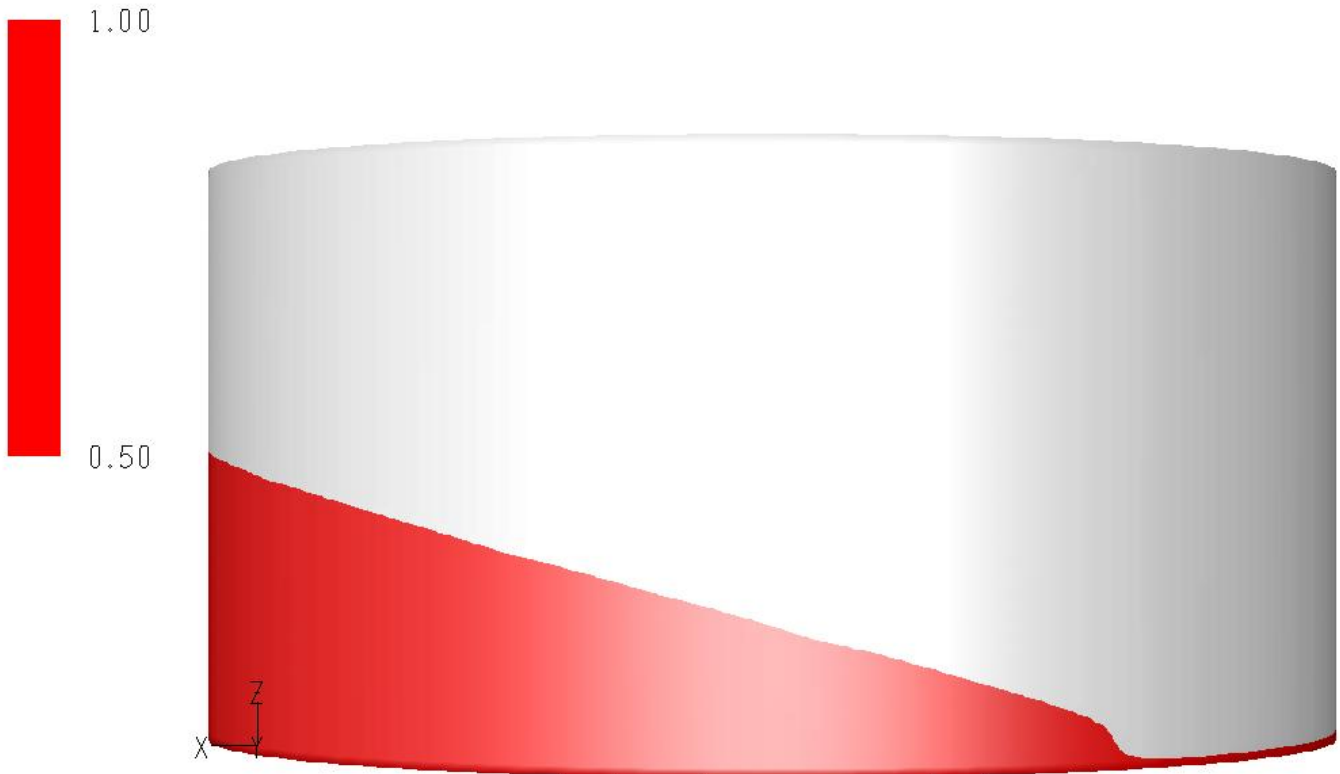

FIGURE 17 - Froude Number 0.2 Free Surface Plot 


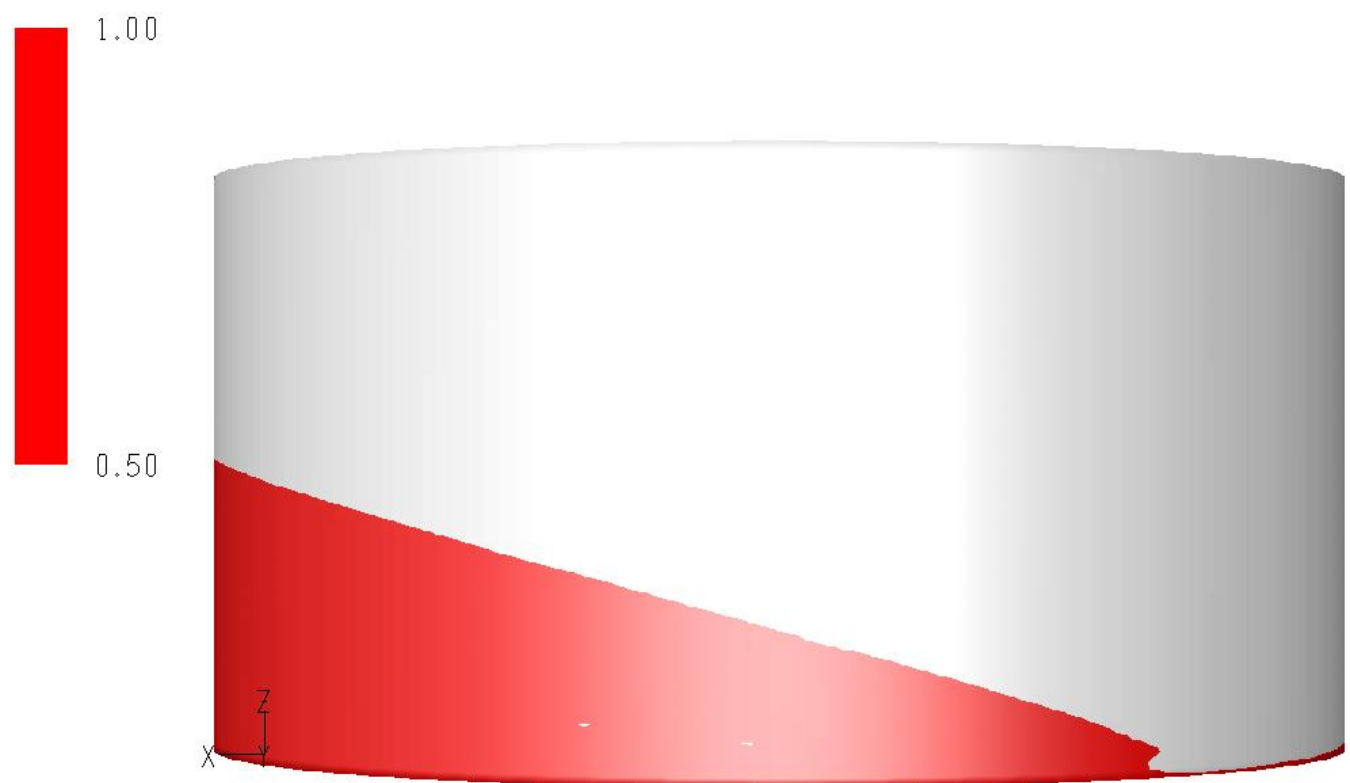

FIGURE 18 - Froude Number 0.3 Free Surface Plot

With the knowledge that that the Froude transition occurs at a Froude Number of $0.25 \pm 0.05$, the cases that were run that fall into the low Froude range consist of the values 0.1 and 0.2 . The Froude Number of 0.2 case was previously described as possessing the vertical wall effect, the high end of the low Froude range. The case for the Froude Number of 0.1, seen in Figure 19, shows that the slope of the leading edge is similar to the slope of the rest of the leading edge of the wave. This signifies that the Froude Number of 0.1 is at the low end of the low Froude range. As only two cases are present in the low Froude range, each representing an extreme, four additional cases at the intervals $0.12,0.14,0.16$, and 0.18 (seen in Figures 20-23) were examined in order to study the change in the low Froude region. These cases show the desired progression of low Froude range. The case for the Froude Number 0.12 has a slope of its leading edge only slightly greater than the 0.1 case. The free surface plot at each progressive case has 
a greater slope of the leading edge until a nearly vertical wall effect becomes present at the 0.16 case. Actually, inspection of the free surface plot for the $0.16,0.18$, and 0.2 cases show 0.16 and 0.18 to be visually identical, with only a slight increase in slope occurring at 0.2. This yields an interesting development as these are the only cases in which identical Froude effects are present.

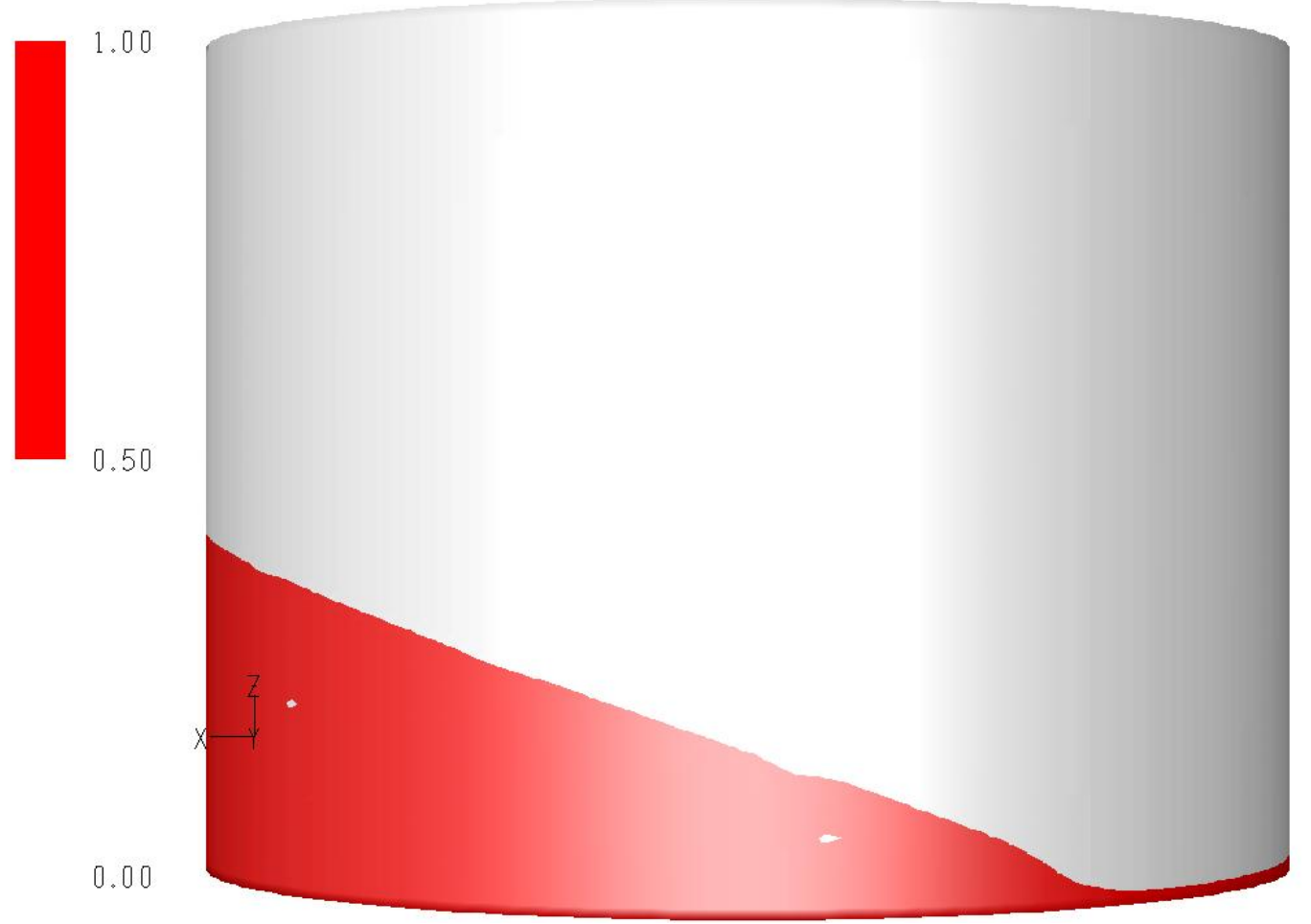

FIGURE 19 - Froude Number 0.1 Free Surface Plot 

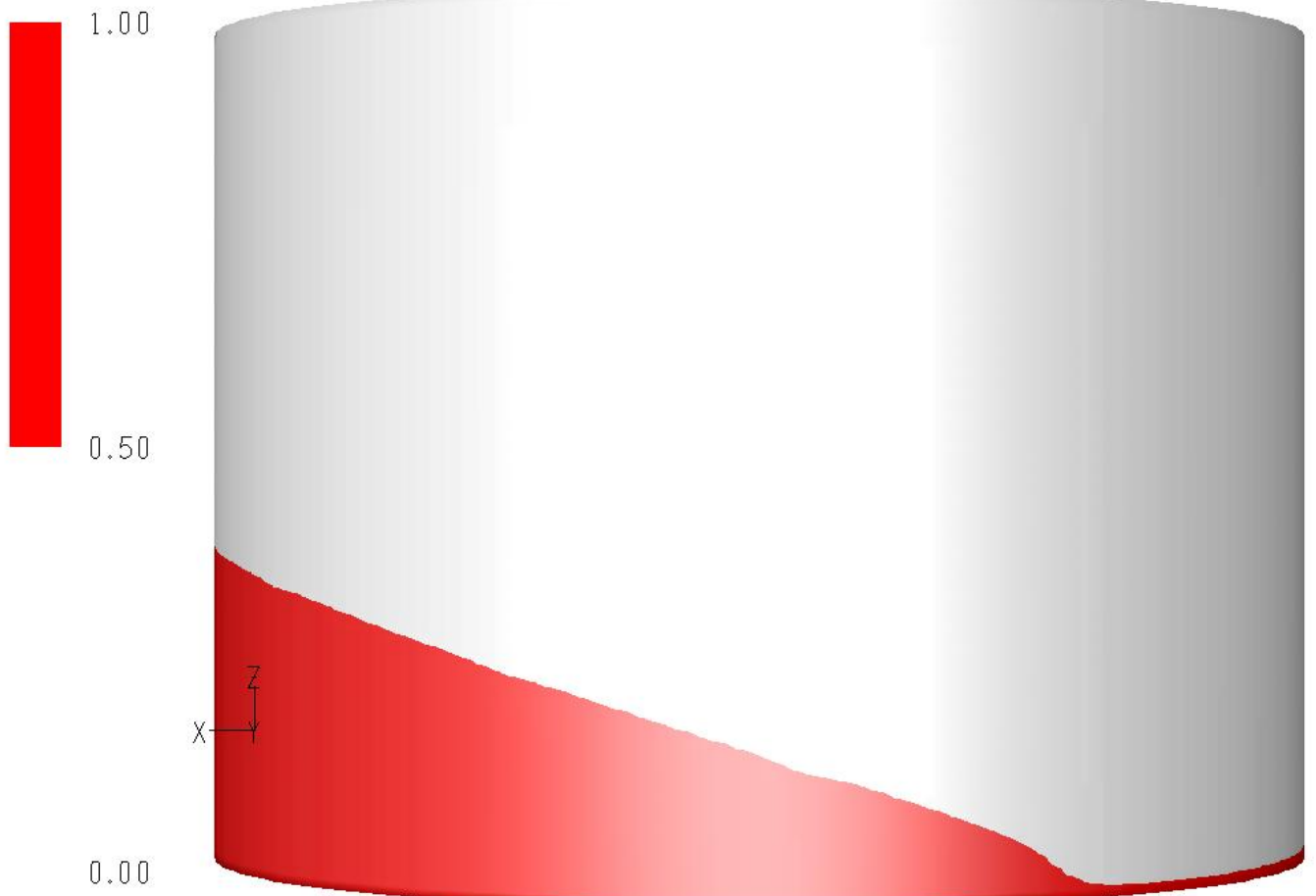

FIGURE 20 - Froude Number 0.12 Free Surface Plot

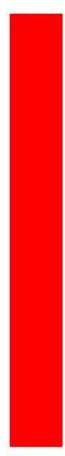

1.00

0.50

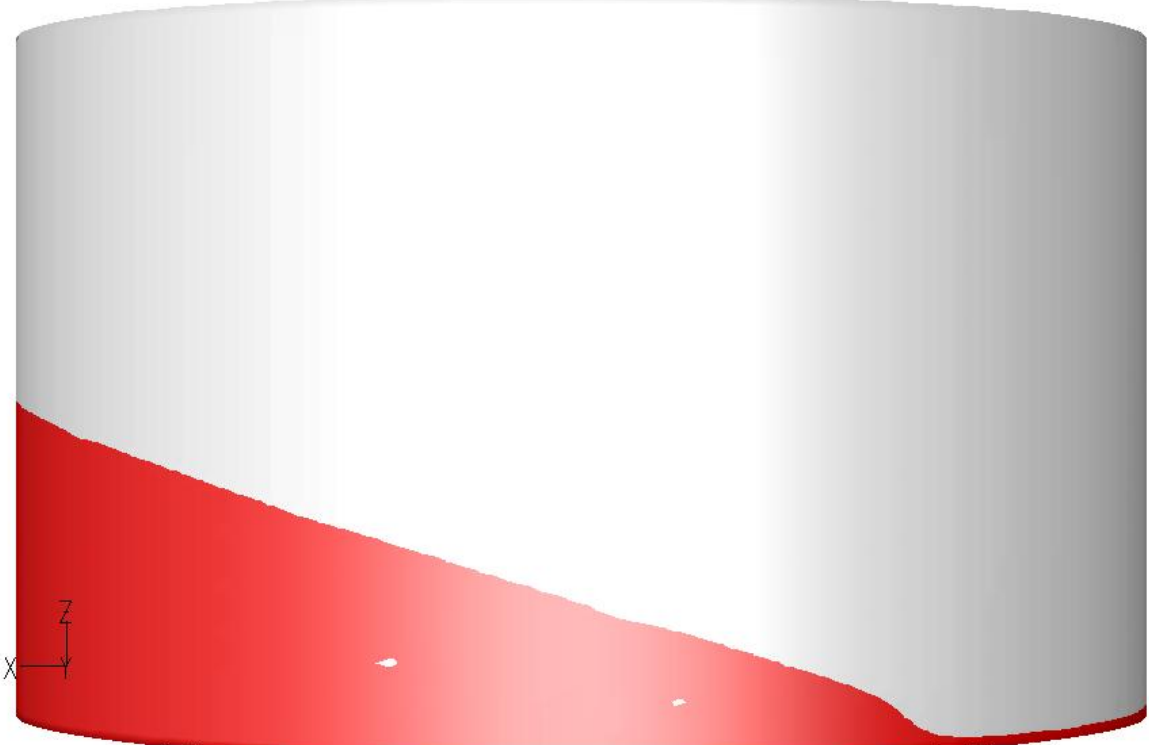

0.00

FIGURE 21 - Froude Number 0.14 Free Surface Plot 

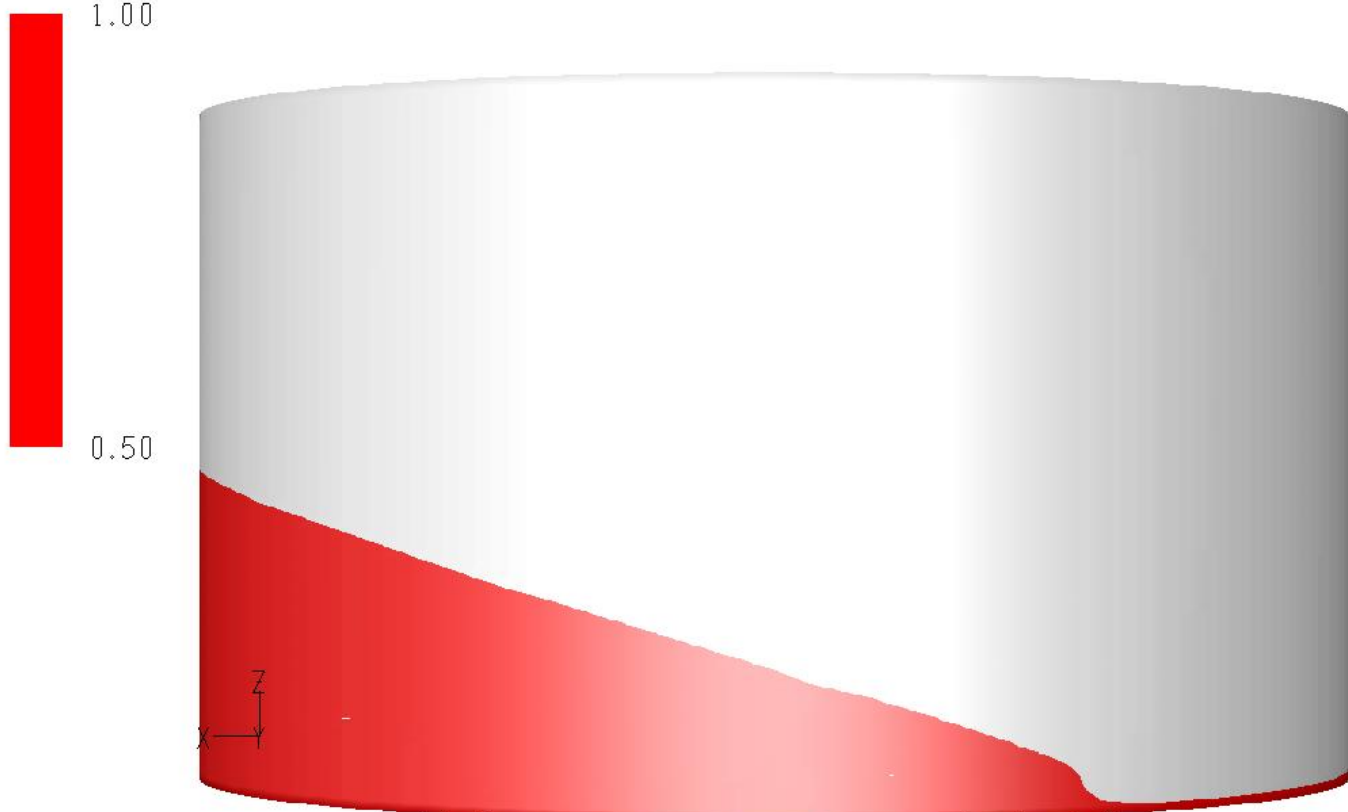

FIGURE 22 - Froude Number 0.16 Free Surface Plot
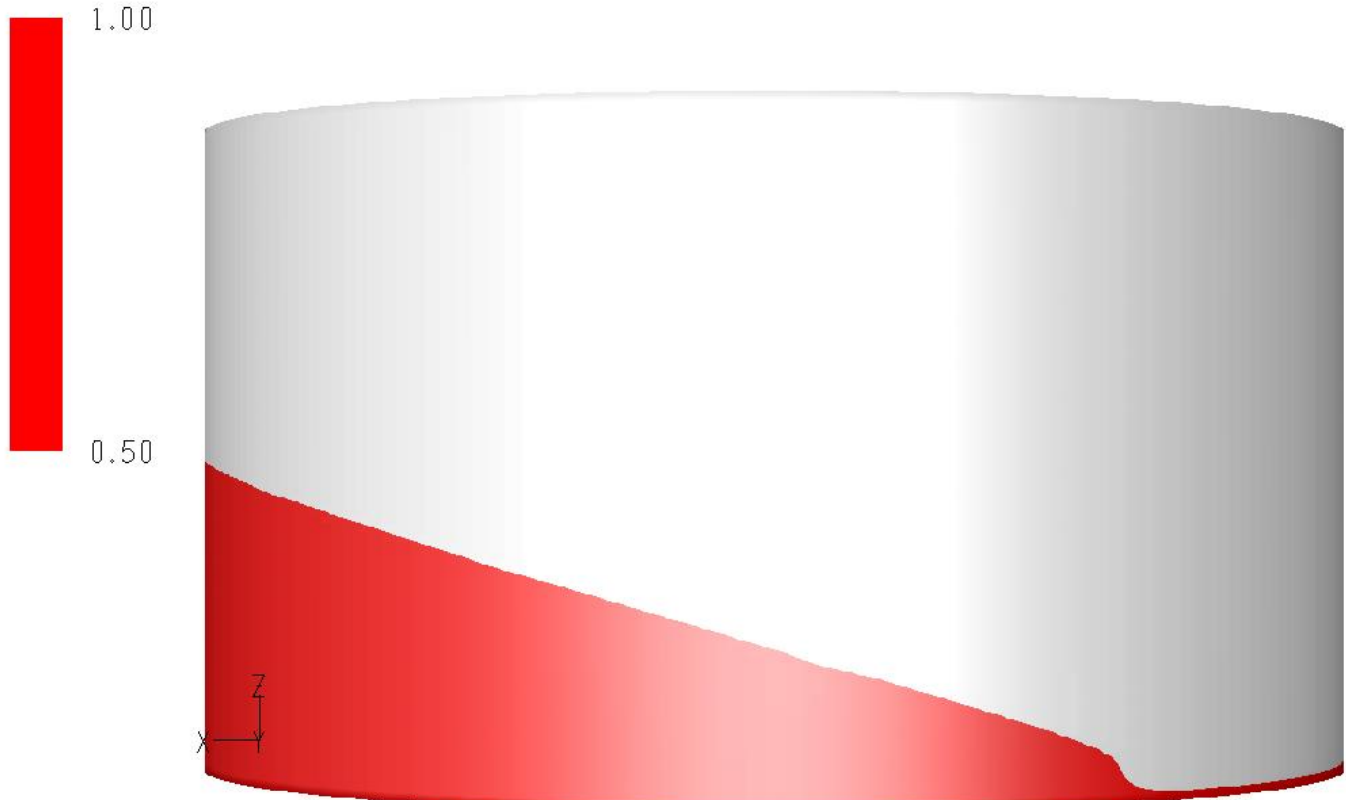

FIGURE 23 - Froude Number 0.18 Free Surface Plot 
The final area of study in our investigation of free surface plots as the Froude number is varied concerns cases in the high Froude range. It has been discussed that overshooting waves develop at the leading edge of the wave in the high Froude range. However, the effect of further increasing the Froude Number on the standing wave has yet to be examined. Figures 18 and 24-27 show the cases in the high Froude range. At a Froude Number of 0.3 , the standing wave is small and makes a small rounded point, with the bottom of the cylinder still wet below it. Increasing to the Froude Number of 0.4, the overshooting wave resembles a small arrowhead point with a dry area below. As the Froude Number is increased to 0.5 , the overshooting wave elongates and develops a rounded profile. When the Froude Number is further increased to 0.7, the overshooting wave elongates further and becomes increasingly thin, resembling a crashing ocean wave. Above 0.7, raising the Froude Number further seems to have minimal effect on the wave at the trailing edge as the wave at the Froude Number of 1.0 resembles that at 0.7 . 


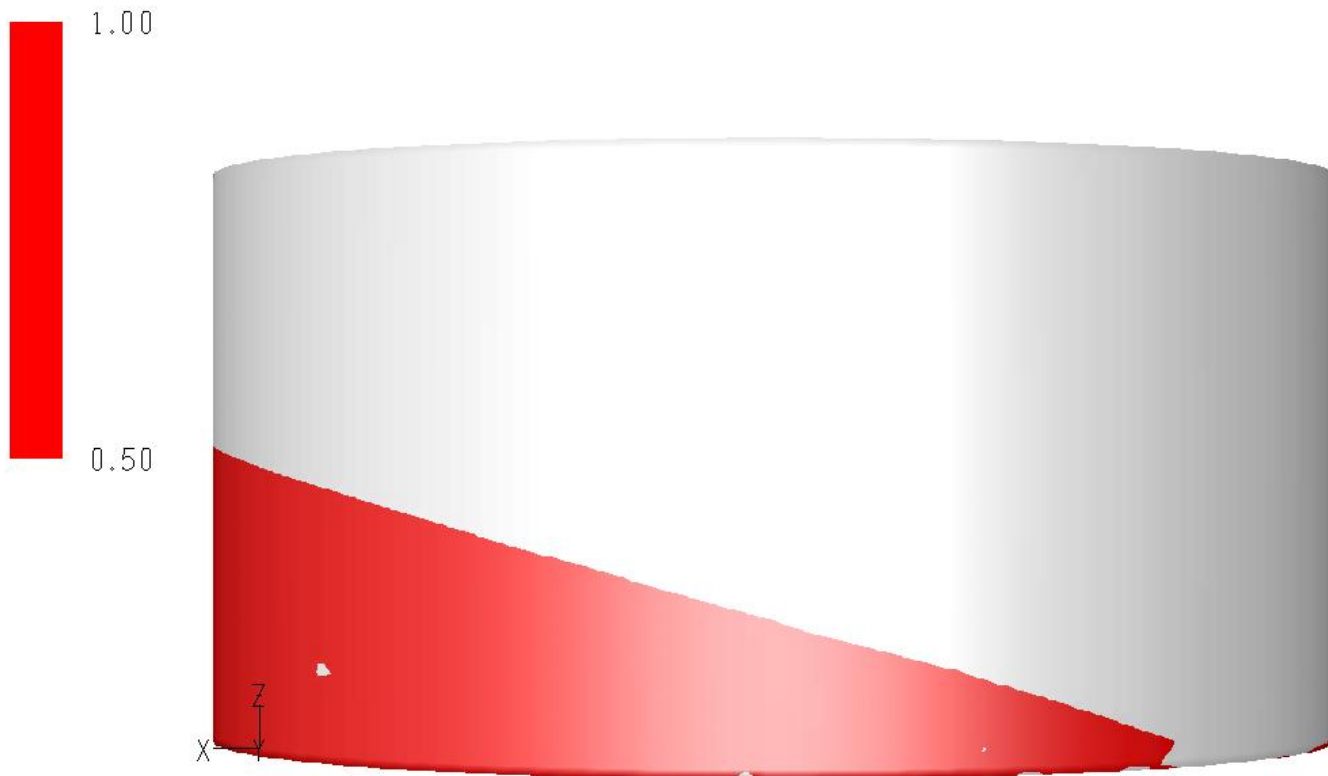

0.00

FIGURE 24 - Froude Number 0.4 Free Surface Plot

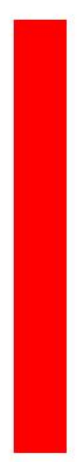

1.00

0.50

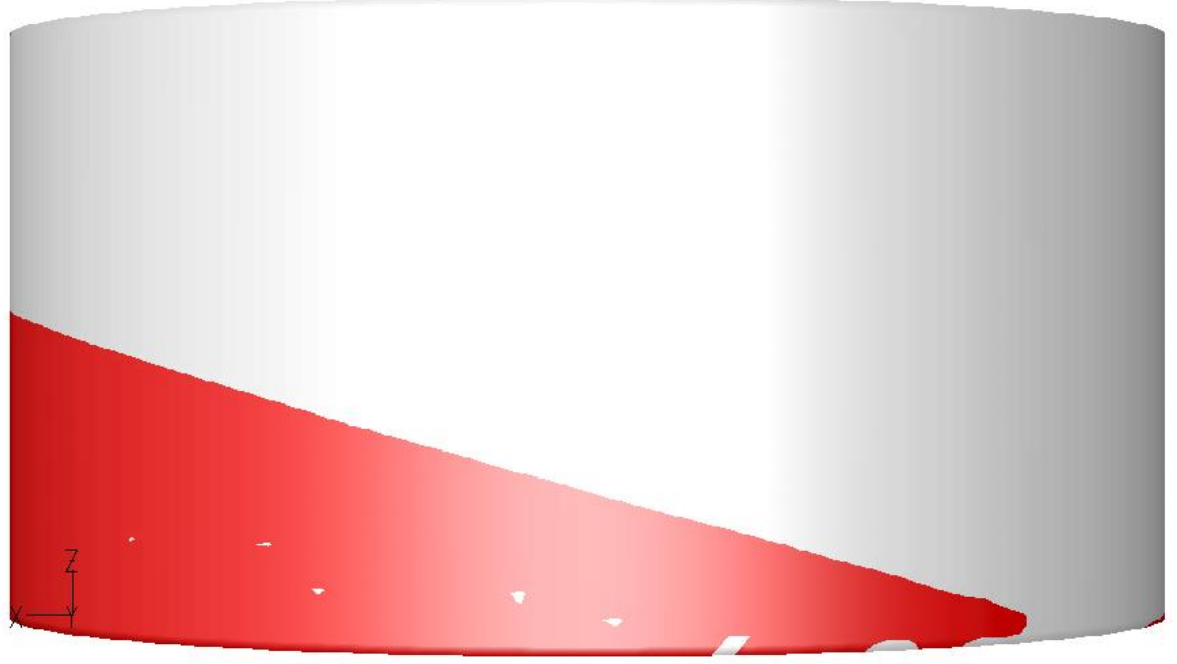

0.00

FIGURE 25 - Froude Number 0.5 Free Surface Plot 


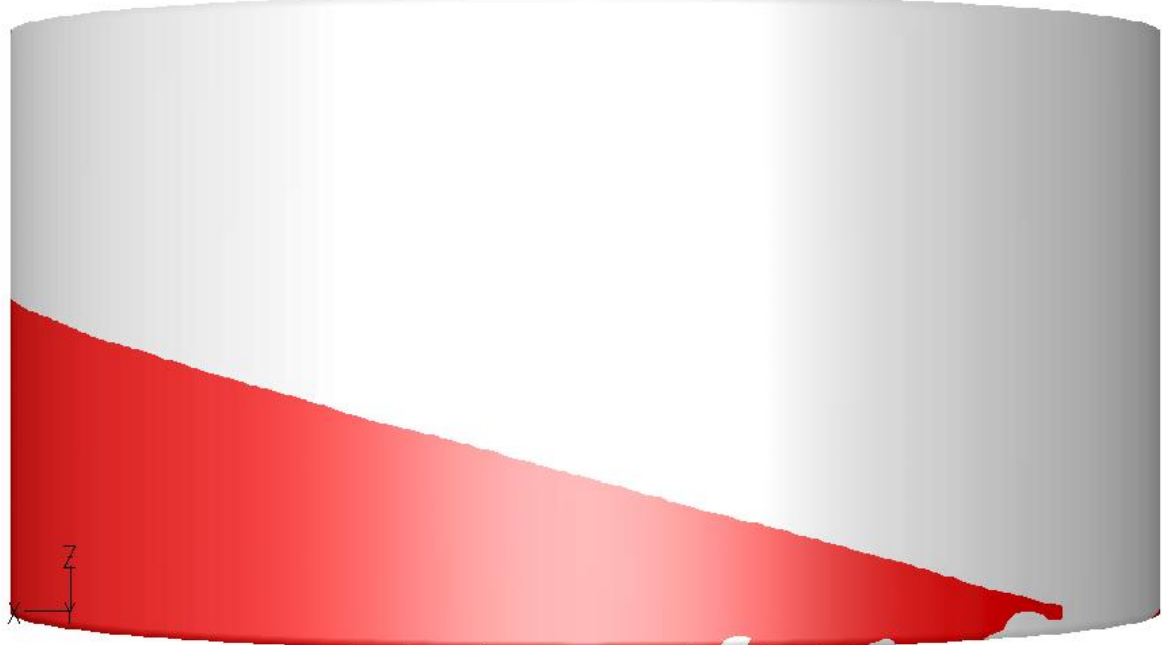

FIGURE 26 - Froude Number 0.7 Free Surface Plot
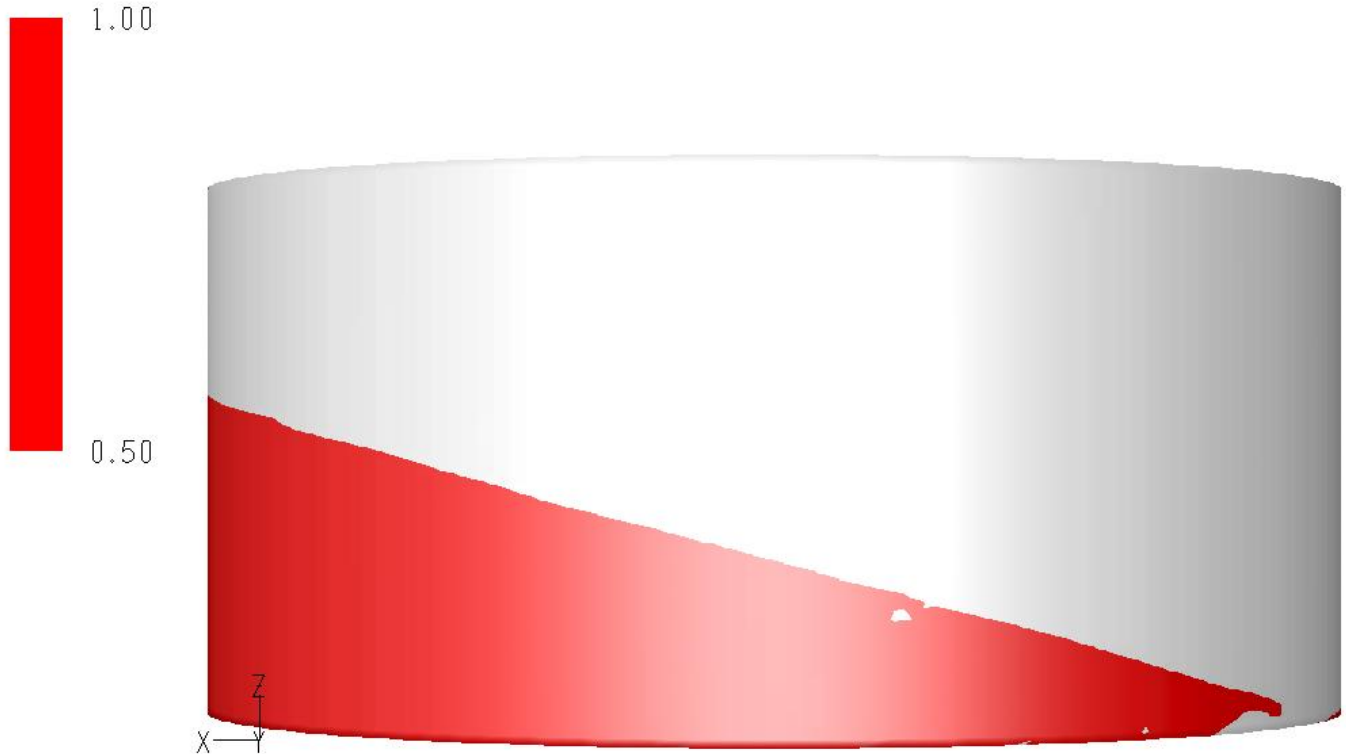

FIGURE 27 - Froude Number 1.0 Free Surface Plot 


\section{Free Surface Plots as a Function of Slope Ratio}

Much like with the Stokes and Froude Numbers, the transition for the Slope Ratio is determined from visual inspection. The area of interest for this set of cases, however, is the trailing edge of the wave. The Slope transition occurs when the free surface intersects the bottom or at the value where the slope of the trailing edge becomes nonuniform and a concave effect develops for high Stokes Numbers. To create the view necessary to inspect this transition, the dish is rotated until the peak of the wave rests at the far right point in the field of view and the wave slopes downward with the highest part of the wave on the right and lowest visible part on the left.

The initial cases of study were for Slope Ratios of 0.1, 0.2, 0.5, and 2.0 for a Stokes Number of 5.0 and a Froude Number of 1.0. The cases for slope ratios of $0.1 \mathrm{a}$ 0.2 produce unexpected free surface plots which will be discussed later. This leaves the cases of Slope Ratios of 0.5 and 2.0 for study of the Slope Transition. These cases are seen in Figures 28 and 29. For a Slope Ratio of 0.5, the trailing edge of the wave shows a smooth slope downward. This signifies that at this Slope Ratio, the case is below the Slope Transition. The case for the Slope Ratio of 2.0 shows a different trend. There is a large visible concavity present on the trailing edge of the wave. Because of this, the Slope Ratio of 2.0 is above the Slope Transition. 

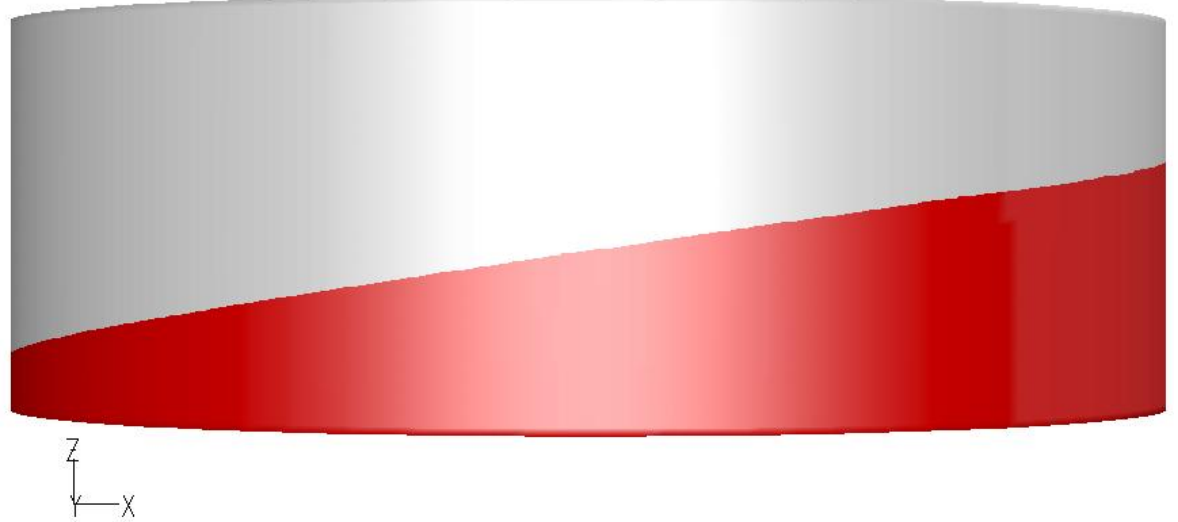

FIGURE 28 - Slope Ratio 0.5 Free Surface Plot
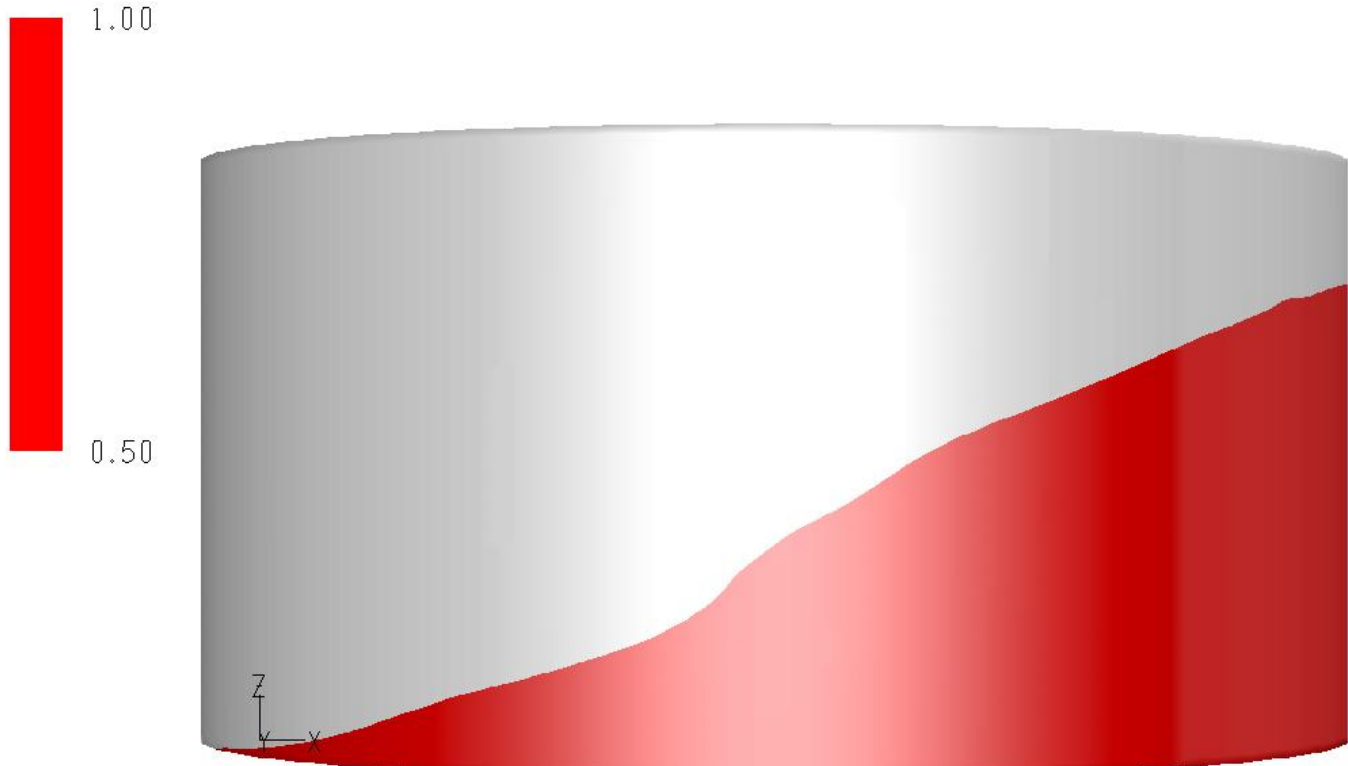

0.00

FIGURE 29 - Slope Ratio 2.0 Free Surface Plot 
Two cases are not adequate enough to realize the beginning of the Slope Ratio effects. No effect is present at 0.5 , while a dominate concavity is evident at 2.0 . To more accurately define the Slope Transition, four additional equally spaced cases are developed for Slope Ratios of $0.8,1.1,1.4$, and 1.7. The free surface plots for these cases are viewed in Figures 30-33.

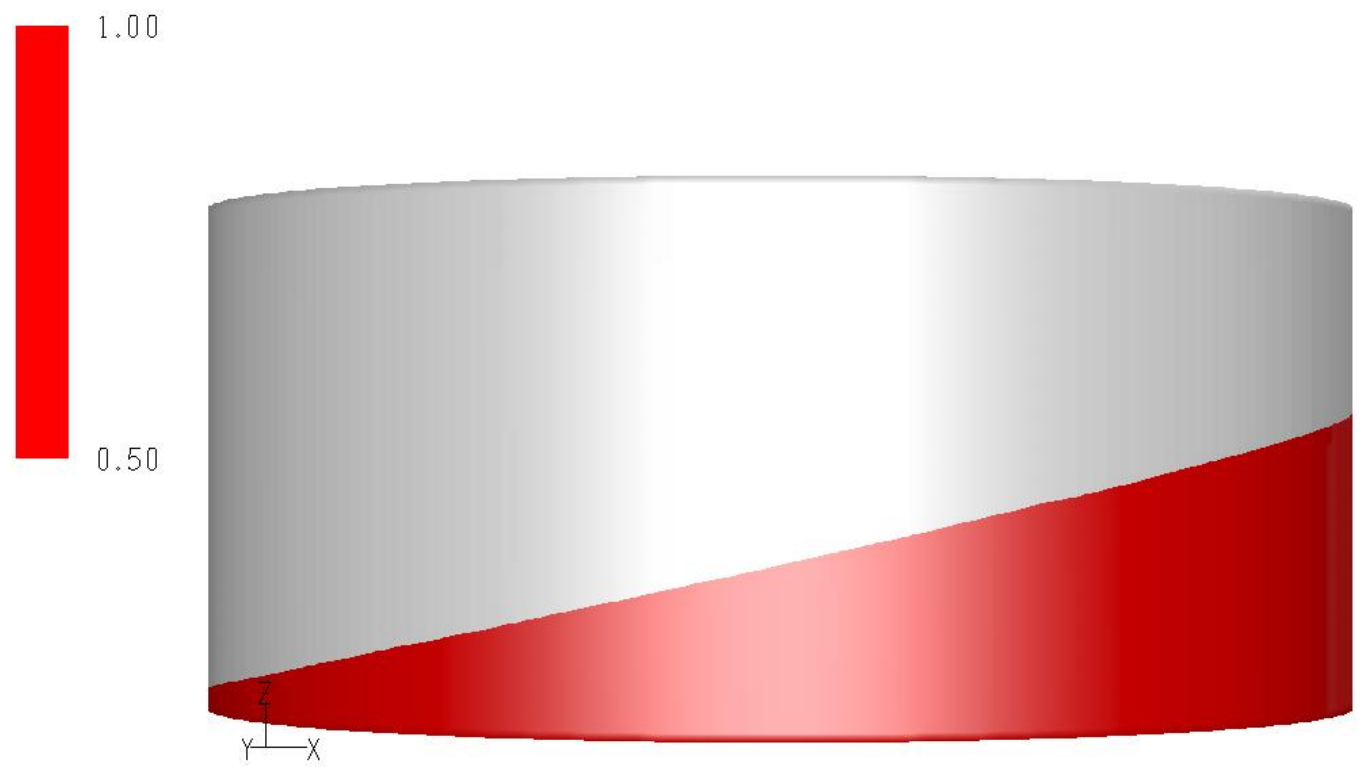

FIGURE 30 - Slope Ratio 0.8 Free Surface Plot 

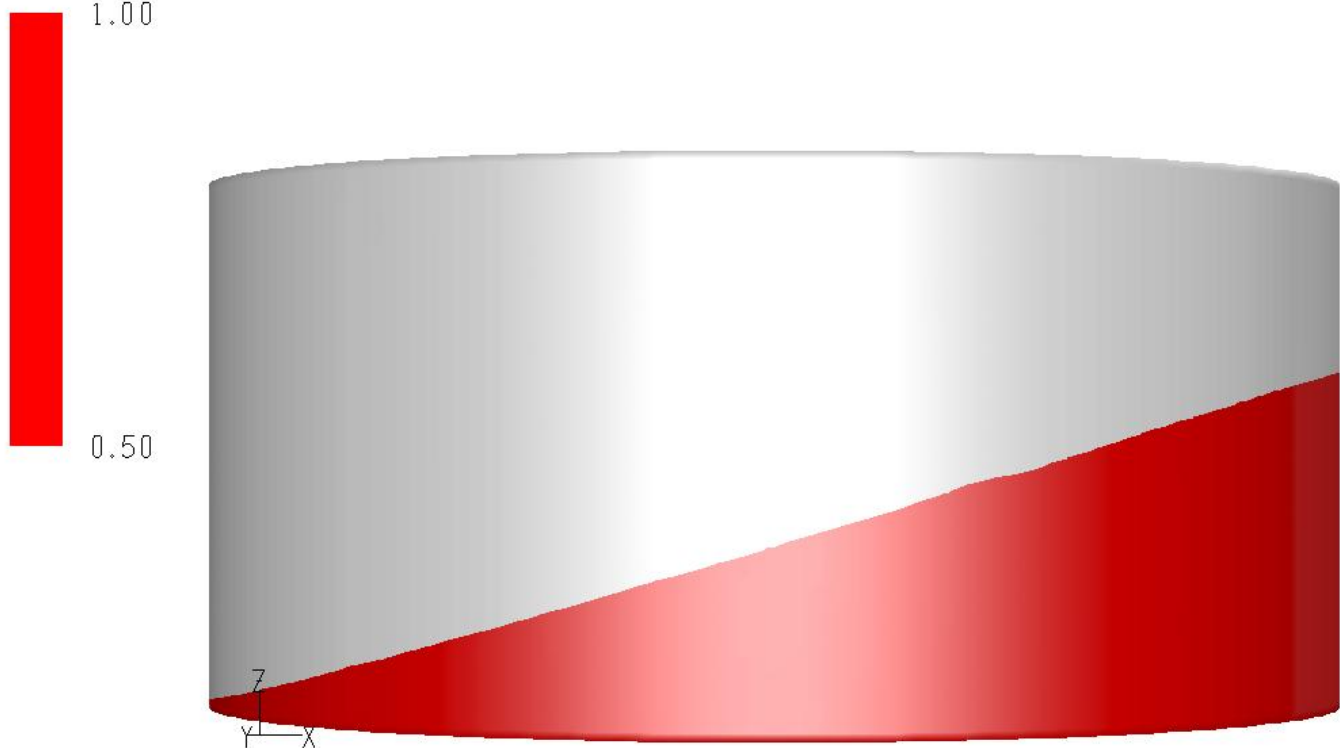

FIGURE 31 - Slope Ratio 1.1 Free Surface Plot
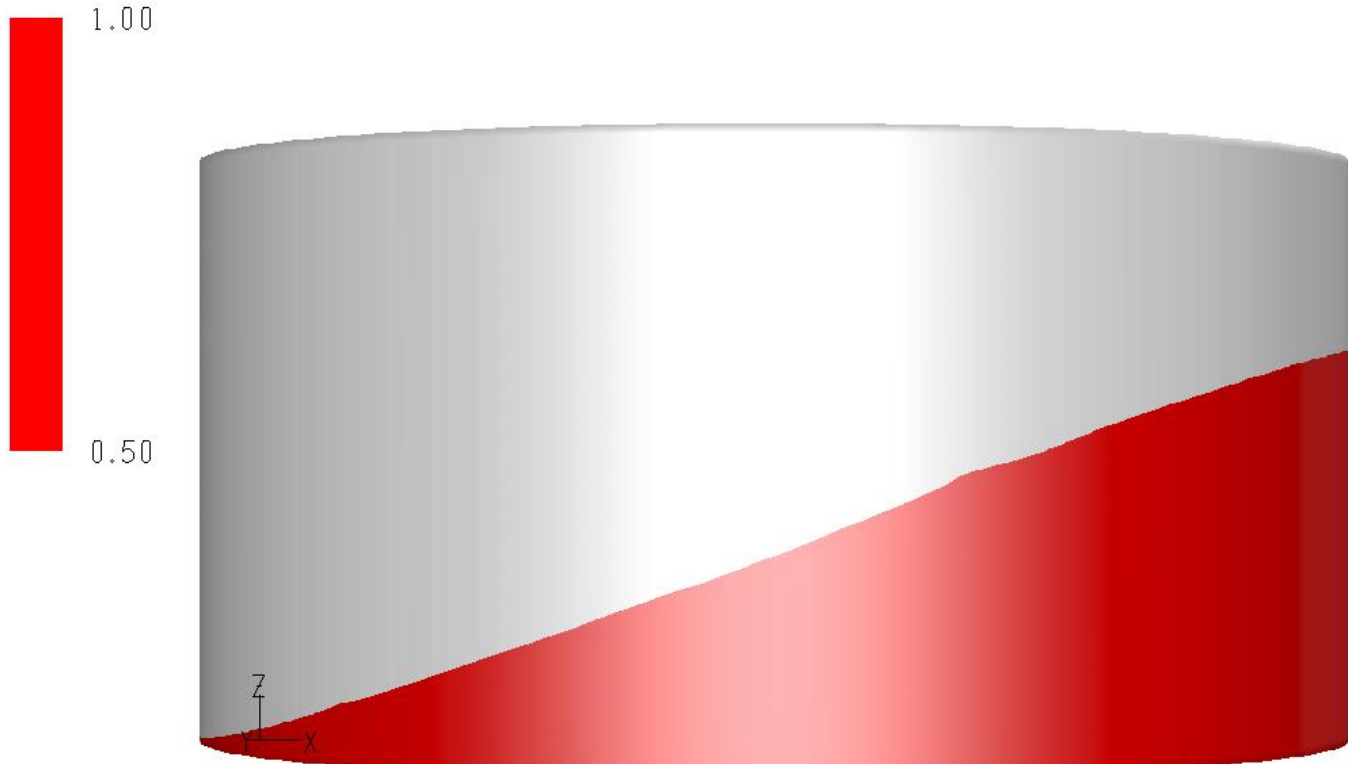

FIGURE 32 - Slope Ratio 1.4 Free Surface Plot 


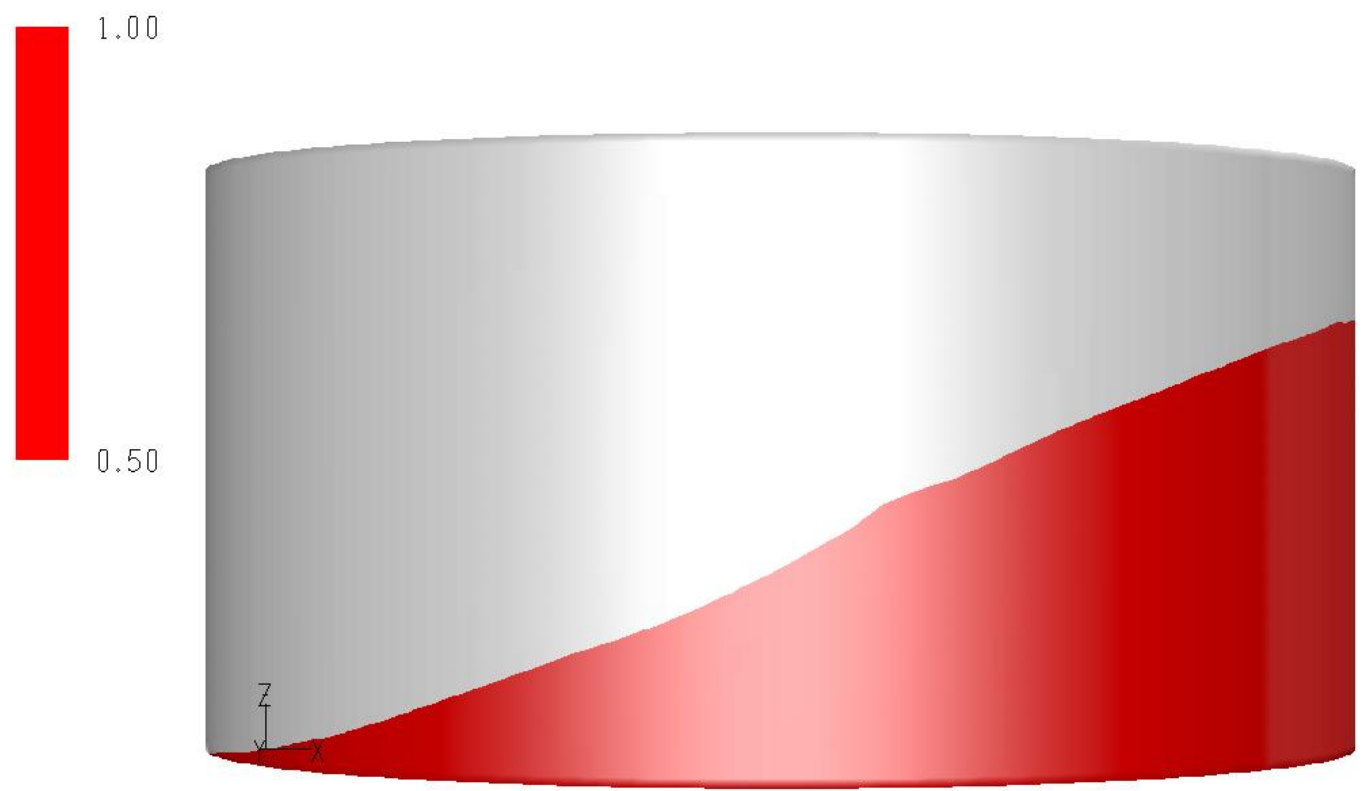

FIGURE 33 - Slope Ratio 1.7 Free Surface Plot

When the four subsequent cases are viewed, the Slope transition is easily seen. The case for the Slope Ratio of 0.8 displays a uniform slope down the trailing edge of the wave. This case looks similar to the case for the Slope Ratio of 0.5, except that the slope of the wave, as expected, is greater. The Slope Ratio of 1.1 shows no sign of concavity, but an interesting effect is present. Roughly a third of the way down the wave from the peak, a small bump appears. This effect is caused by the drying of the dish at the trailing edge. Figures 34 shows that at the Slope Ratio of 0.8 the dish is wet around its complete base, yet Figure 35 shows that a small dry area has developed at the trailing edge of the wave. The bump is created as a result of the drying phenomena at the trailing edge of the wave. As the dry area is created, the bump effect is created as gravity tries to force the trailing edge to cover as much of the dry area as possible. To compensate, when an exceedingly large dry area is present, the trailing edge becomes convex as the trailing 
edge thins in an effort to cover as much dry space as possible. The Slope Ratio of 1.4 shows the first visual sign of concavity, with the concavity beginning after the bump effect. As expected, the case for the Slope Ratio of 1.7 has a more defined concavity

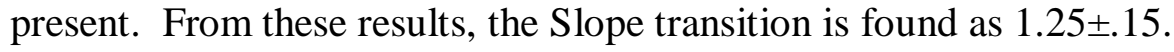

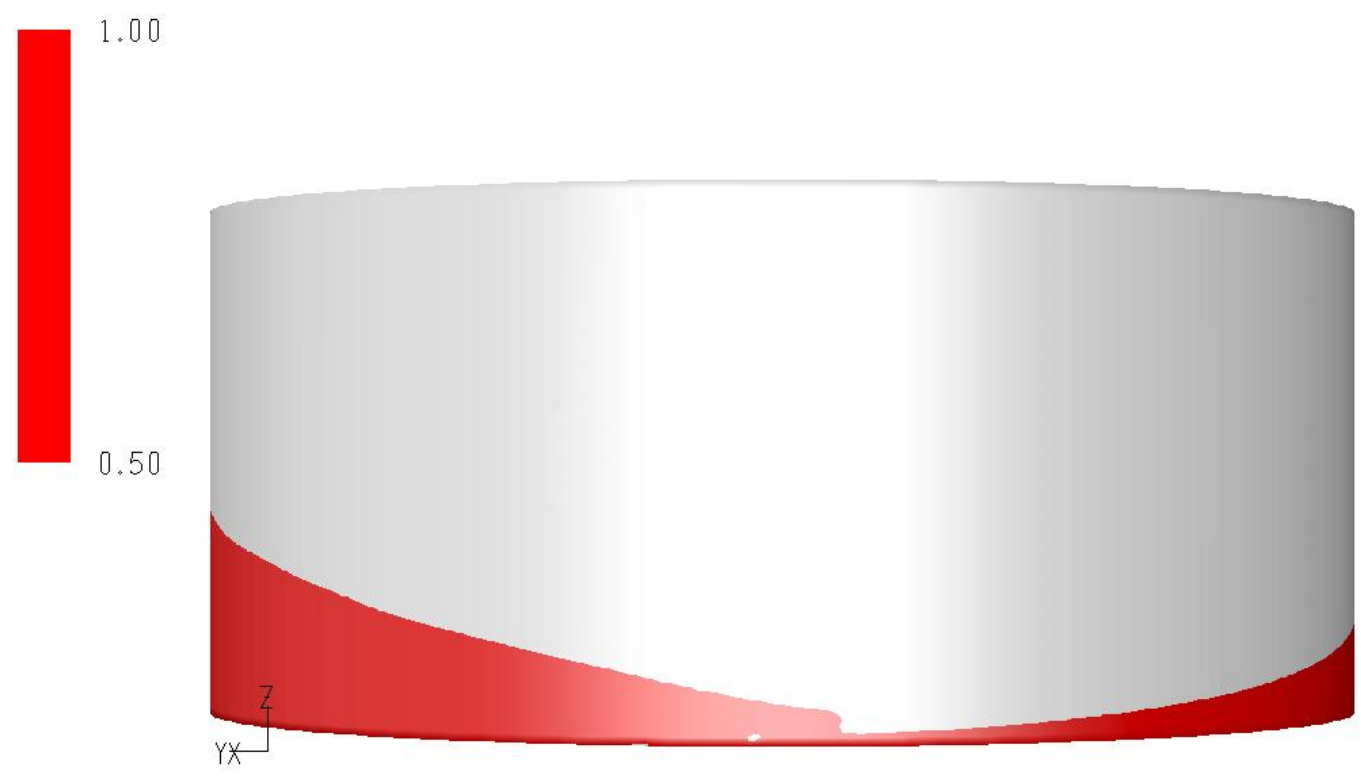

FIGURE 34 - Slope Ratio 0.8 Back Side Free Surface Plot 

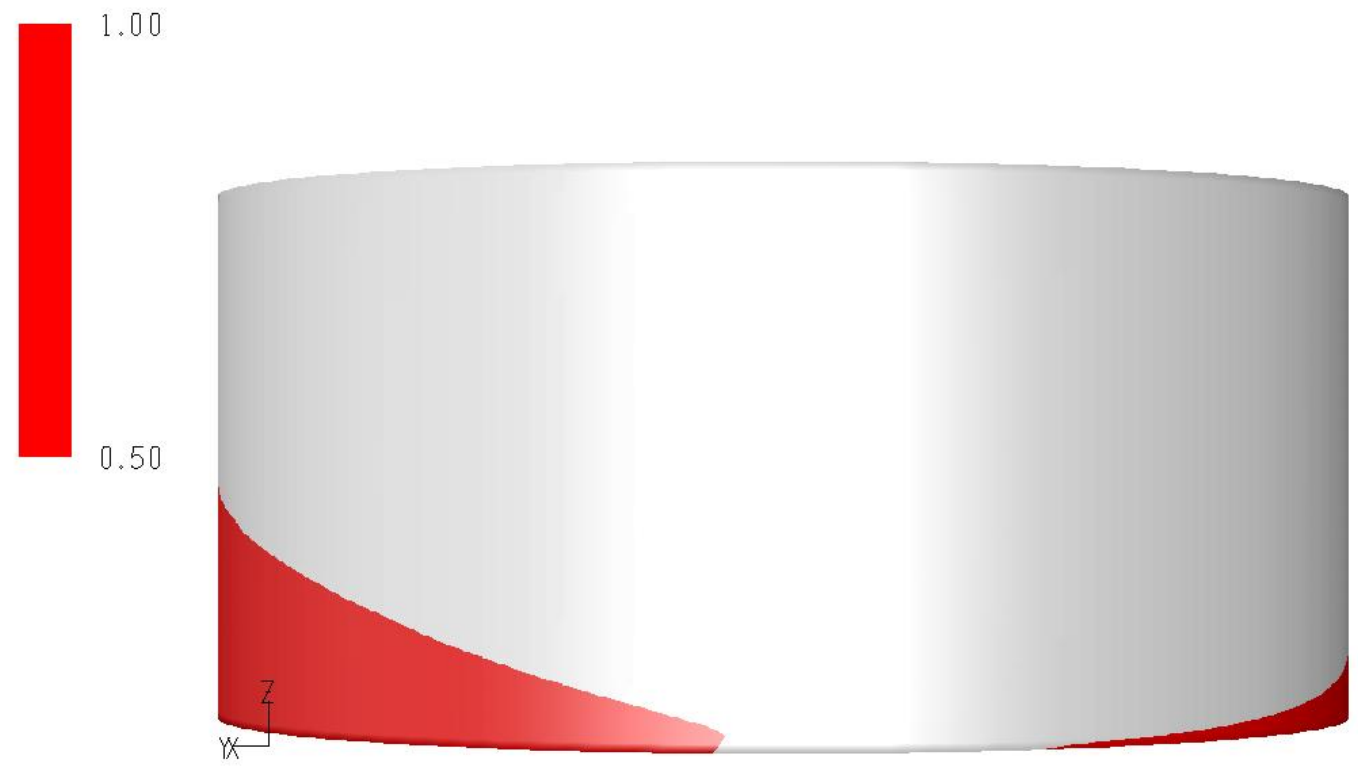

FIGURE 35 - Slope Ratio 1.1 Back Side Free Surface Plot

The cases for the Slope Ratios of 0.1 and 0.2 have free surface plots that were completely unexpected. The front and back views of these cases are seen in Figures 3639. Instead of having a wave with one continuous peak, the two cases have four peaks of varying sizes. More interesting is that the smaller of the two peaks, on the left in Figures 36 and 38, correspond in location to the singular peak in the other studied cases. This phenomenon is most likely caused by the effects of a dimensionless parameter other than the four studied. When consulting Tables I-III to find a concrete difference between these two cases and the others, these two cases have the largest diameter and fluid height of all those studied. The additional centrifugal forces that arise as a result could be a cause of the additional waves. 


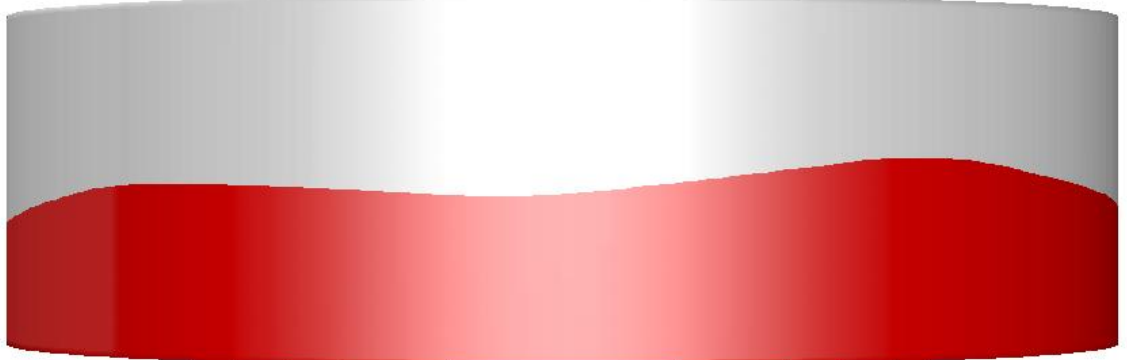

$y \longrightarrow x$

FIGURE 36 - Slope Ratio 0.1 Front Side Free Surface Plot
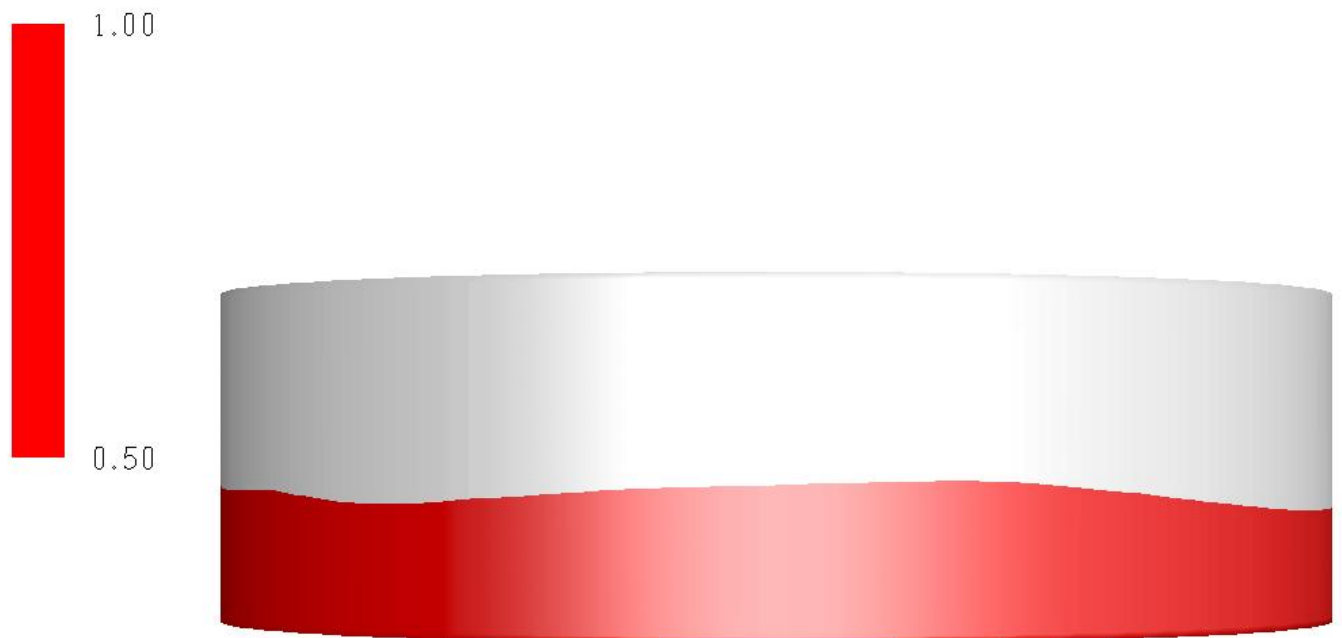

$\varlimsup_{X-Y}^{Z}$

FIGURE 37 - Slope Ratio 0.1 Back Side Free Surface Plot 

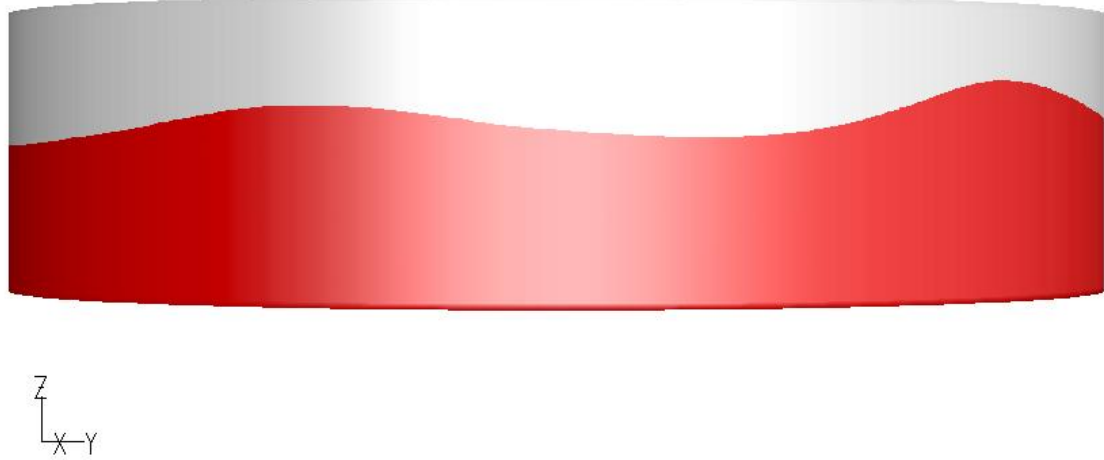

FIGURE 38 - Slope Ratio 0.2 Front Side Free Surface Plot
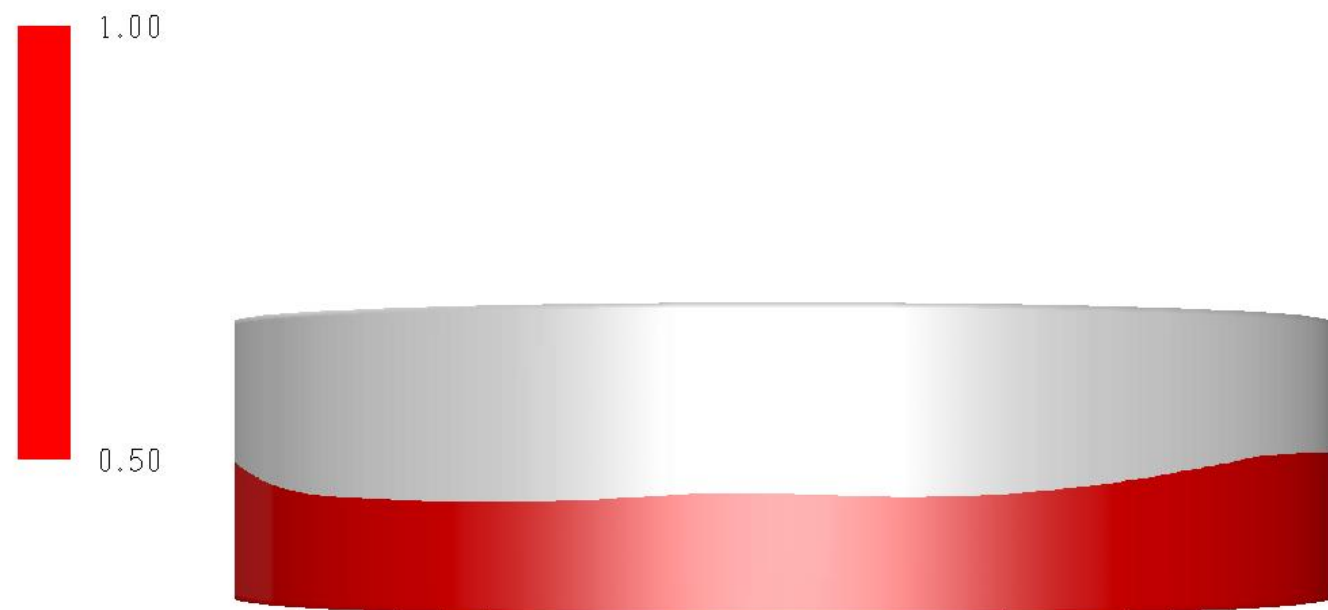<smiles>[3H][V][3H]</smiles>

FIGURE 39 - Slope Ratio 0.2 Back Side Free Surface Plot 


\section{B. Analysis of Wall Shear Stress Contours}

\section{Wall Shear Stress Contours as a Function of Stokes Number}

In studying wall shear stress (WSS) contours, a feature of paramount importance is the maximum WSS on the bottom. It is the best quantitative indicator of the differences in varying dimensionless parameters since each WSS contour is more or less significantly different. Values for maximum bottom WSS as the Stokes Number is varied are shown in TABLE V for a Froude Number of 1.0 and a Slope Ratio of 1.0. Maximum bottom WSS values increase as Stokes Number is increased ranging from $8.85^{*} 10^{-}$

${ }_{8}^{8} \frac{d y n e s}{\mathrm{~cm}^{2}}$ at a Stokes number of 1.0 to $16.41 \frac{\text { dynes }}{\mathrm{cm}^{2}}$ at a Stokes Number of 10. These values fall well within the ranges reported in other literature [(Levesque, 1985), (Ley, 1989), (Dardik, 2005)].

\section{TABLE V}

MAXIMUM BOTTOM WALL SHEAR STRESS VS. STOKES NUMBERS

\begin{tabular}{|c|c|}
\hline Stokes Number & Wall Shear Stress \\
\hline 1.0 & $8.85^{*} 10^{-8}$ \\
\hline 2.0 & 2.52 \\
\hline 3.0 & 4.27 \\
\hline 4.0 & 6.55 \\
\hline 4.2 & 7.66 \\
\hline 4.4 & 8.26 \\
\hline 4.6 & 9.15 \\
\hline 4.8 & 9.50 \\
\hline 5.0 & 9.54 \\
\hline 10 & 16.41 \\
\hline
\end{tabular}

To observe how the Stokes transition affects the maximum bottom WSS, values are plotted in Figure 40. A noticeable jump in WSS is visible between 4.0 and 4.2 where the Stokes transition lies. After the transition from 4.2 to 5.0, it looks as if the WSS is leveling off. Although when the Strokes Number of 10 is examined, it is plainly visible 
that shear does not level off at increasingly high Stokes Numbers. When the overall picture is examined, however, it looks as though linear slopes are present on either side of the transition except for a localized increase in the slope at the Stokes Transition. A comparison of the Stokes data collected from FLUENT to Equation (6) and Equation (7) is seen in Figure 41. It is difficult to gauge the trends in Figure 41 as the scale is skewed from Equation (7) rising exponentially, so the point for The Stokes Number of 10 is removed in Figure 42. Equation (6) follows the experimental data relatively close from a low Stokes value to a high Stokes value, falling closer further away from the Stokes transition. Visual inspection of Figure 42 shows that Equation (6) follows the data more closely before the Stokes transition. This is interesting as the equation is derived for high Stokes Numbers. Additionally, in the high Stokes range, Equation (6) surpasses the maximum observed bottom wall shear stress values. Since Equation (6) represents WSS values at the center and not maximum values, it is surprising that these values are greater than the maximum values found experimentally. Equation (7) follows somewhat closely to the experimental until around the Stokes Number 5.0, upon which time the equation goes off toward infinity. This fits with the derived conditions for Equation (7) as it is derived for low Stokes values. Further inaccuracies in these equations could arise from the fact that they are derived for low Froude Numbers and low Slope Ratios. These dimensionless parameters are at a medium value for these cases. 


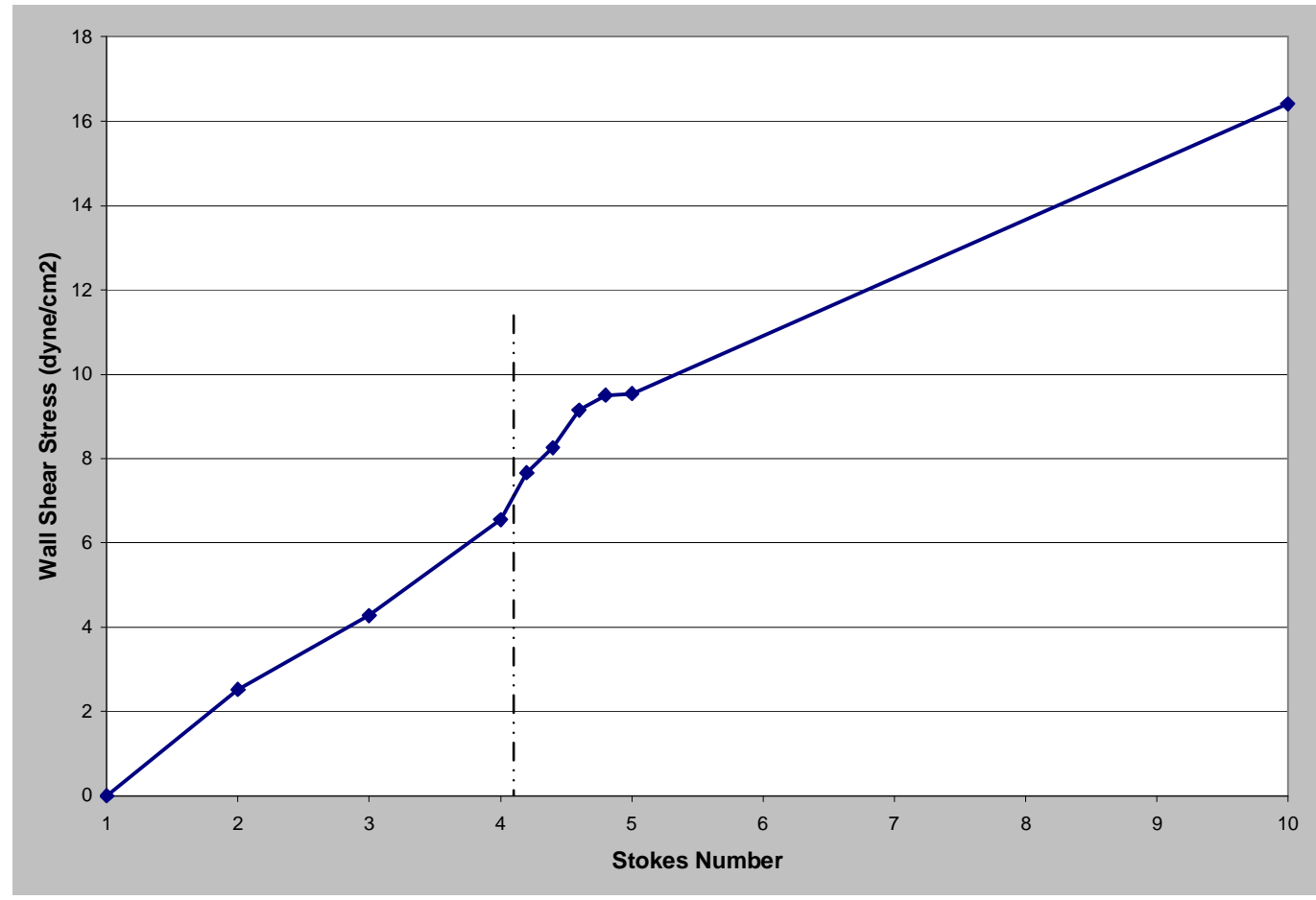

FIGURE 40 - Maximum Bottom Wall Shear Stress versus Stokes Number

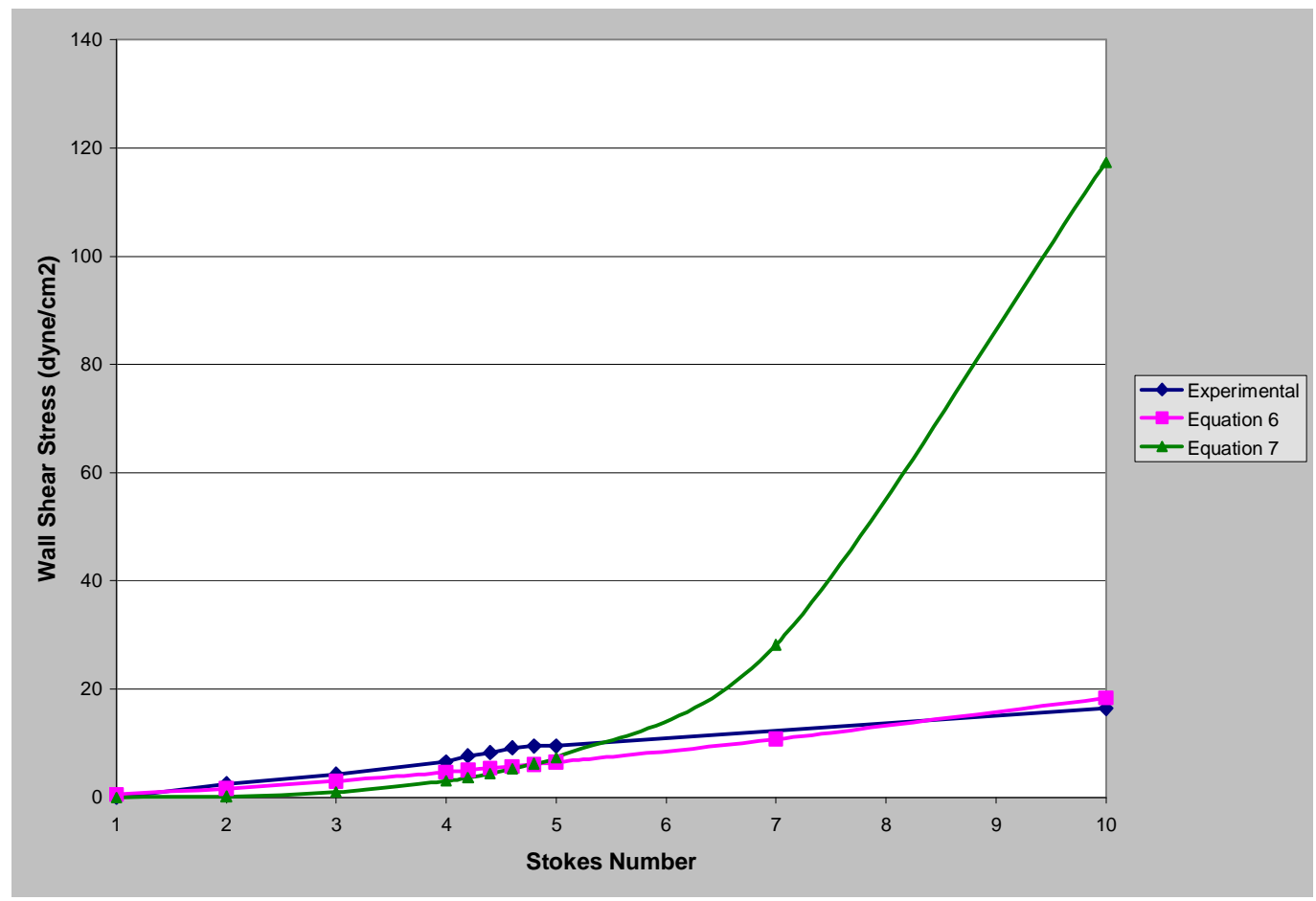

FIGURE 41 - Stokes Number Data Comparison to Equations 


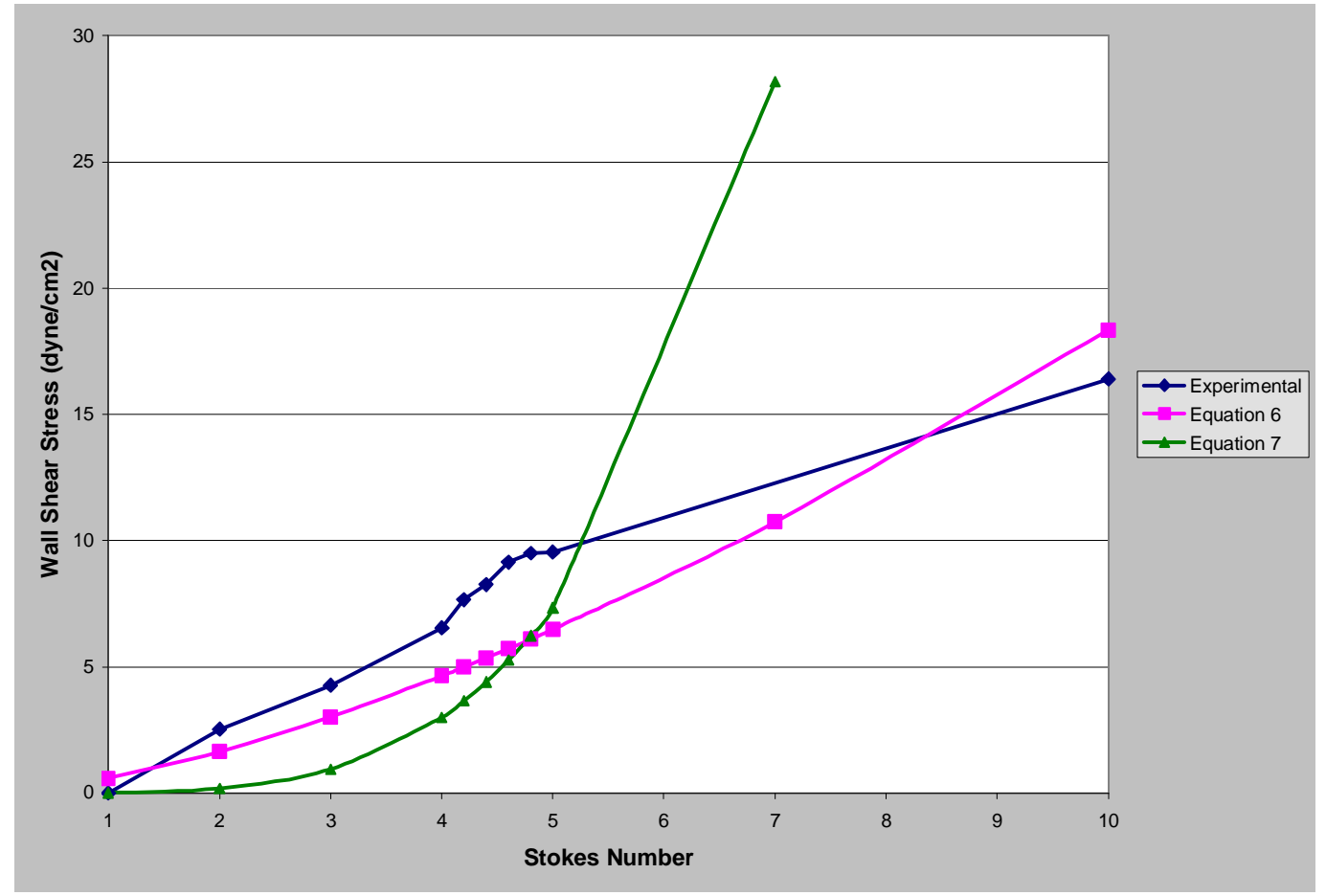

FIGURE 42 - Modified Stokes Number Data Comparison to Equations

Now that the effects of maximum WSS have been investigated, the shape of the actual contours will be analyzed to determine any trends. The Stokes Number of 1.0 showed negligible values of bottom WSS and as a result, Figure 43 looks different than any other WSS contour as the maximum WSS region is in the center of the cylinder with most of the outer region of the dish in the low WSS range. This unusual effect resulted as the case was run at a low orbital speed with a thin initial liquid height; Figure 7 shows that the wave remains essentially flat. From Stokes Numbers of 2.0 through 10, a steady transition of WSS contours is visible. For the Stokes Number of 2.0, seen in Figure 44, a region of high WSS develops near the outside edge of the dish that resembles a comet with its tail following behind. The region of high WSS is located directly below the leading edge of the wave. For a Stokes Number of 3.0, seen in Figure 45, the area of high WSS near the outer edge of the dish is more elongated and has a wavy shape with a 
rounded front as opposed to a triangular shape with a flat front on at a Stokes Number of 2.0. This difference is based upon differing Froude effects: a Stokes Number of 2.0 is in the low Froude range while a Stokes Number of 3.0 is in the high Froude range. These free surface plots are seen in Figures 46 and 47. Also, a second egg shaped region of medium WSS develops directly in from of the leading edge of the wave, roughly $60 \%$ out from the center; this effect is caused by the trailing edge of the wave. At the Stokes Number of 4.0, seen in Figure 48, the region of high WSS becomes much larger and exhibits a greater amount of irregularity. The region of medium WSS has an obvious separation from the high WSS region at a Stokes Number of 3.0, but at a Stokes Number of 4.0 the two regions have begun to merge together. The small circular areas of low WSS are caused by small pockets of air trapped in the liquid region. The Stokes Numbers of 4.2 and 4.4, seen in Figures 49 and 50, follow the same pattern as the Stokes Number of 4.0. The area of high WSS near the outer edge of the dish continues to grow larger and more irregular, while the region of medium WSS moves closer toward the leading edge of the wave. At the Stokes Numbers of 4.6, 4.8, and 5.0, seen in Figures 51 through 53, the region of high WSS grows so large that irregularities begin to merge together and at the Stokes Number of 5.0, only residual waviness is present. The area of medium WSS continues to become closer to the region of high WSS and by the Stokes Number of 5.0, they have almost merged. At the Stokes Number of 10, seen in Figure 54, the region of high WSS extends more than three quarters of the way around the outer edge. Interestingly, the region of medium WSS prevalent in the cases at lower Stokes Numbers is absent from this contour. It seems as though there is no visible effect of the Stokes transition in the WSS contours. 

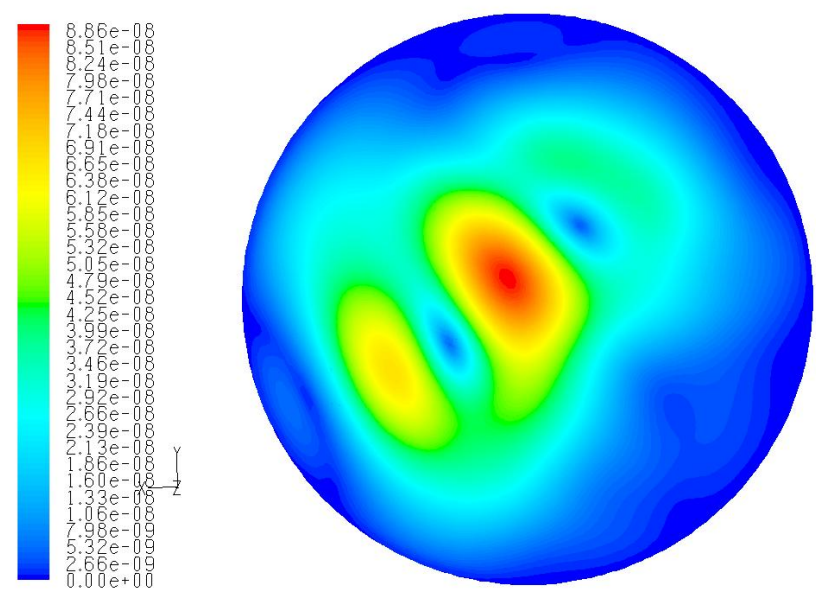

FIGURE 43 - Stokes Number 1.0 Wall Shear Stress Contour
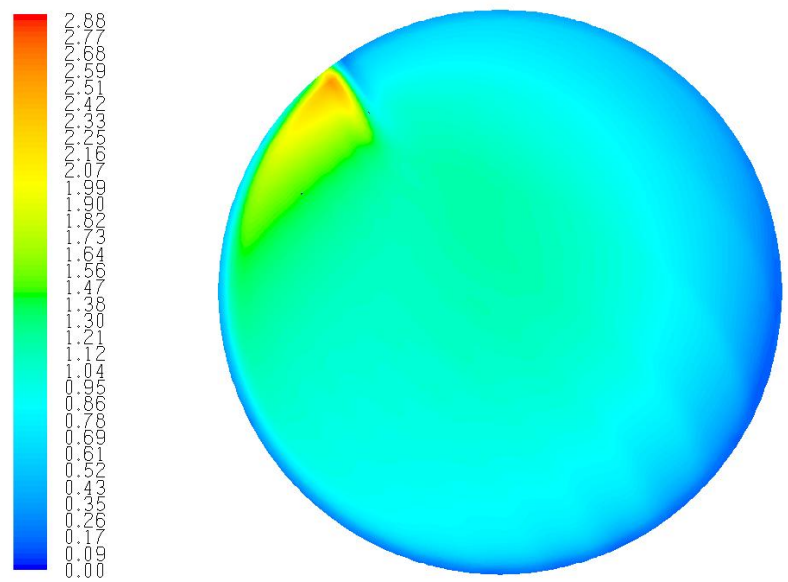

FIGURE 44 - Stokes Number 2.0 Wall Shear Stress Contour
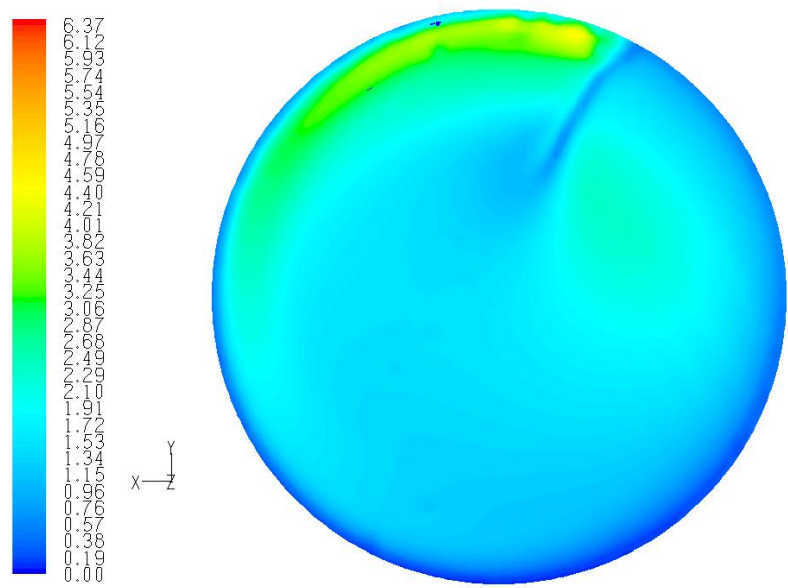

FIGURE 45 - Stokes Number 3.0 Wall Shear Stress Contour 

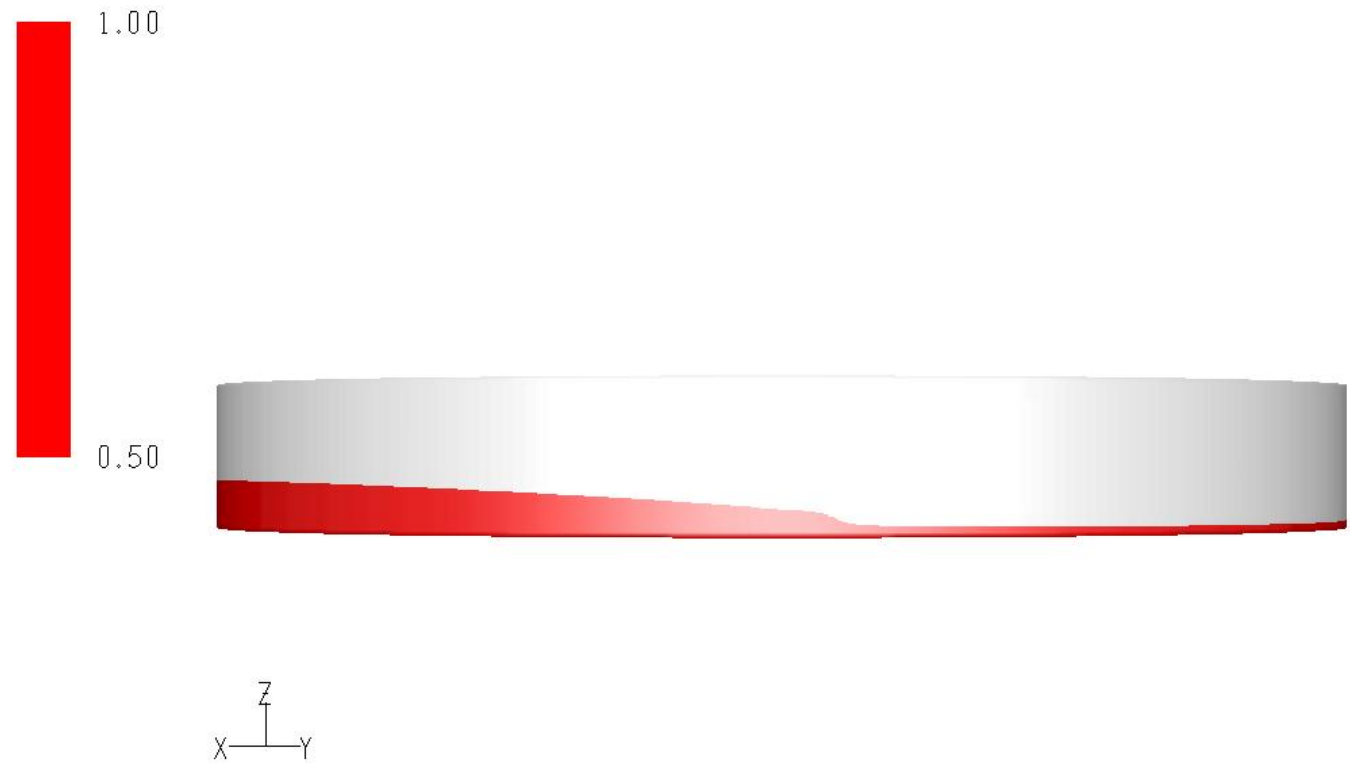

0.00

FIGURE 46 - Stokes Number 2.0 Free Surface Plot with Low Froude Effect

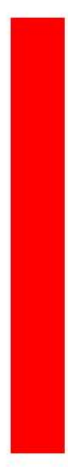

1.00

0.50

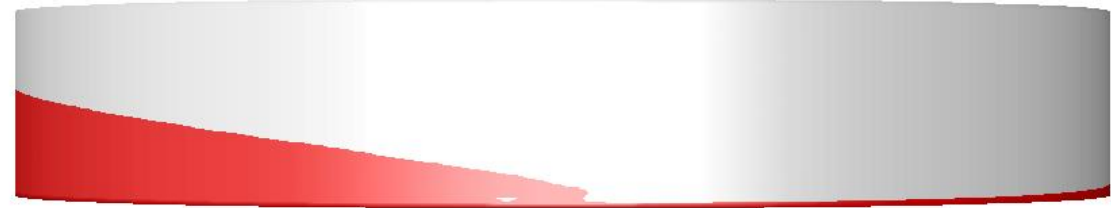

$$
x y
$$

0.00

FIGURE 47 - Stokes Number 3.0 Free Surface Plot with High Froude Effect 


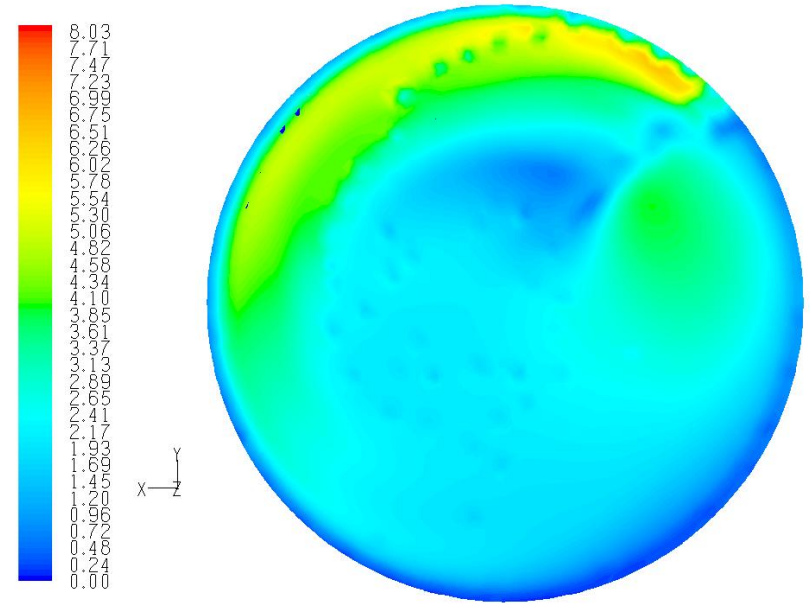

FIGURE 48 - Stokes Number 4.0 Wall Shear Stress Contour

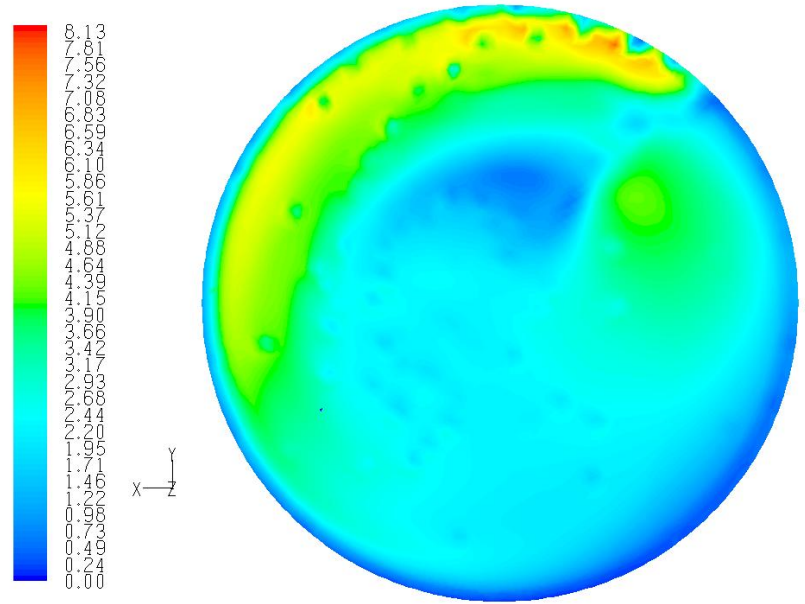

FIGURE 49 - Stokes Number 4.2 Wall Shear Stress Contour

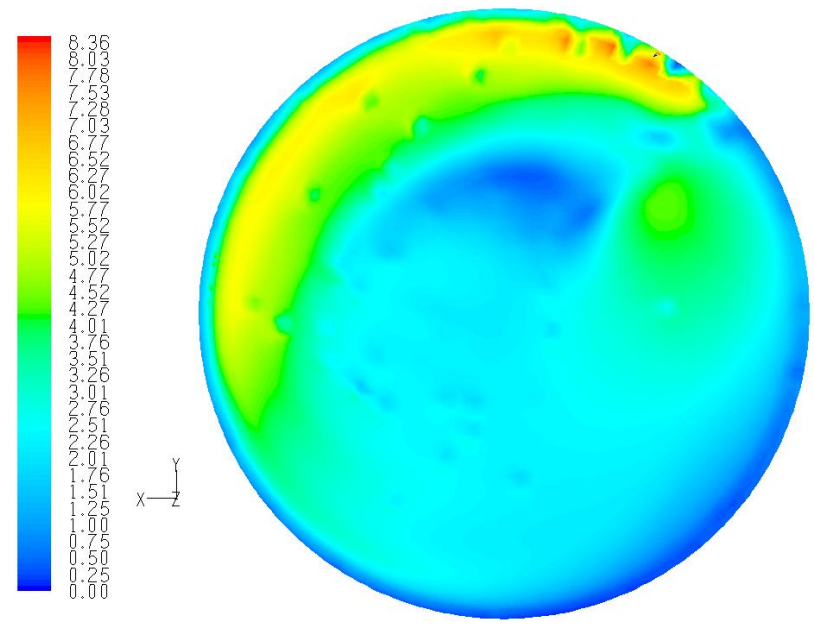

FIGURE 50 - Stokes Number 4.4 Wall Shear Stress Contour 


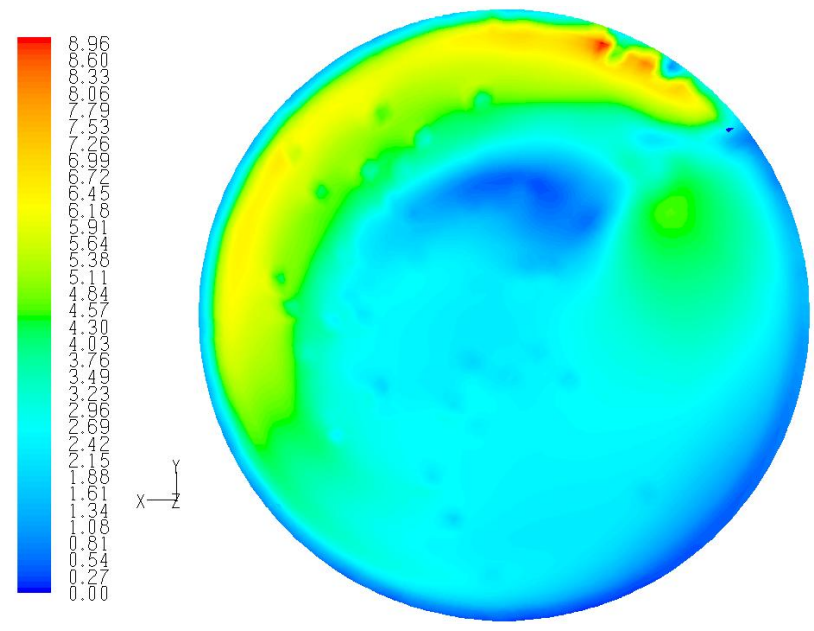

FIGURE 51 - Stokes Number 4.6 Wall Shear Stress Contour

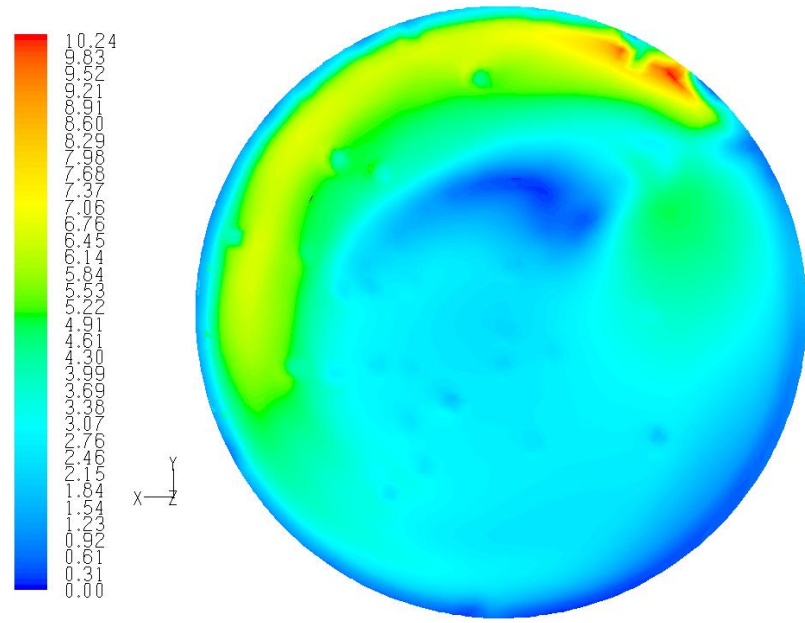

FIGURE 52 - Stokes Number 4.8 Wall Shear Stress Contour

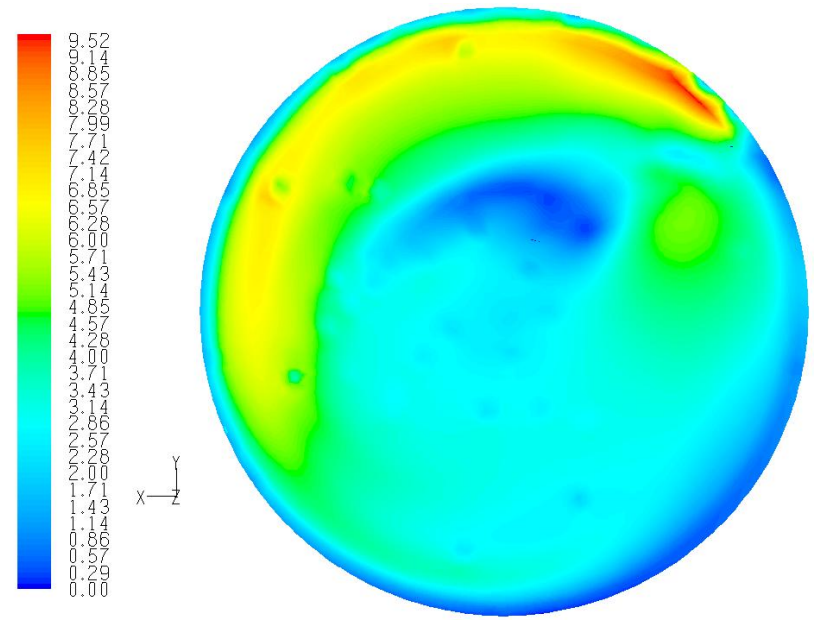

FIGURE 53 - Stokes Number 5.0 Wall Shear Stress Contour 


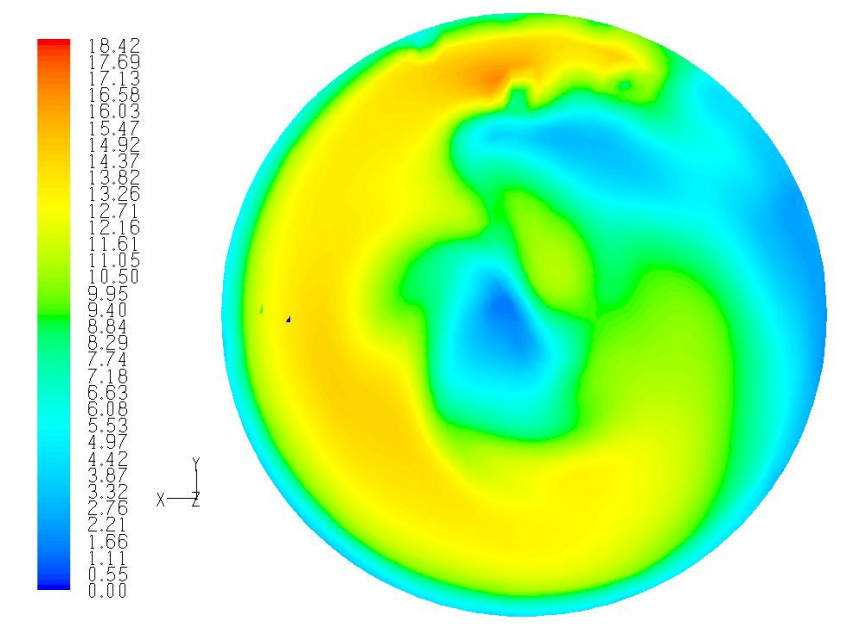

FIGURE 54 - Stokes Number 10 Wall Shear Stress Contour

\section{Wall Shear Stress Contours as a Function of Froude Number}

The first item studied in the investigation of WSS contours as Froude Number is varied is the maximum bottom WSS. Values for maximum bottom WSS as the Froude Number is varied are shown in TABLE VI for a Stokes Number of 5.0 and a Slope Ratio of 1.0. Maximum bottom WSS values increase as Froude Number is increased ranging from $2.41 \frac{d y n e s}{\mathrm{~cm}^{2}}$ at a Froude number of 0.1 to $9.54 \frac{d y n e s}{\mathrm{~cm}^{2}}$ at a Froude Number of 1.0.

TABLE VI

MAXIMUM BOTTOM SHEAR STRESS VS. FROUDE NUMBERS

\begin{tabular}{|c|c|}
\hline Froude Number & Wall Shear Stress \\
\hline 0.1 & 2.41 \\
\hline 0.12 & 2.44 \\
\hline 0.14 & 2.77 \\
\hline 0.16 & 3.18 \\
\hline 0.18 & 3.18 \\
\hline 0.2 & 3.34 \\
\hline 0.3 & 4.48 \\
\hline 0.4 & 4.52 \\
\hline 0.5 & 5.14 \\
\hline 0.7 & 7.35 \\
\hline 1.0 & 9.54 \\
\hline
\end{tabular}


To examine how the Froude transition affects the maximum bottom WSS, a plot was prepared as seen in Figure 55. At the Froude transition between 0.2 and 0.3 , a localized increase in the slope occurs. Between 0.16 and 0.5 the slope is significantly low except that the Froude transition has the effect of localized increase in slope of maximum WSS. After 0.5 the maximum bottom WSS begins to rise at a steady rate. Another deviation is seen between 0.1 and 0.12 . This change however is likely due to the change in WSS leveling off at extremely low values of the Froude Number. A leveling off is expected because all the Froude cases have the same, moderately quick, orbital speed. A high orbital speed ensures that the WSS will never reach values near zero. A comparison of the Froude data collected from FLUENT to Equation (6) and Equation (7) is seen in Figure 56. Equation (6) has a flat slope and shows no correlation to the experimental maximum WSS data. However, Equation (7) follows the basic pattern of the experimental Froude data although lower in magnitude through the range observed. Both equations are derived for low Froude thought Equation (7) fits the trend of the data well in both high and low Froude regions. As Equation (7) represents shear at the center, it seems a feasible estimation of shear for the entirety of the Froude range observed. 


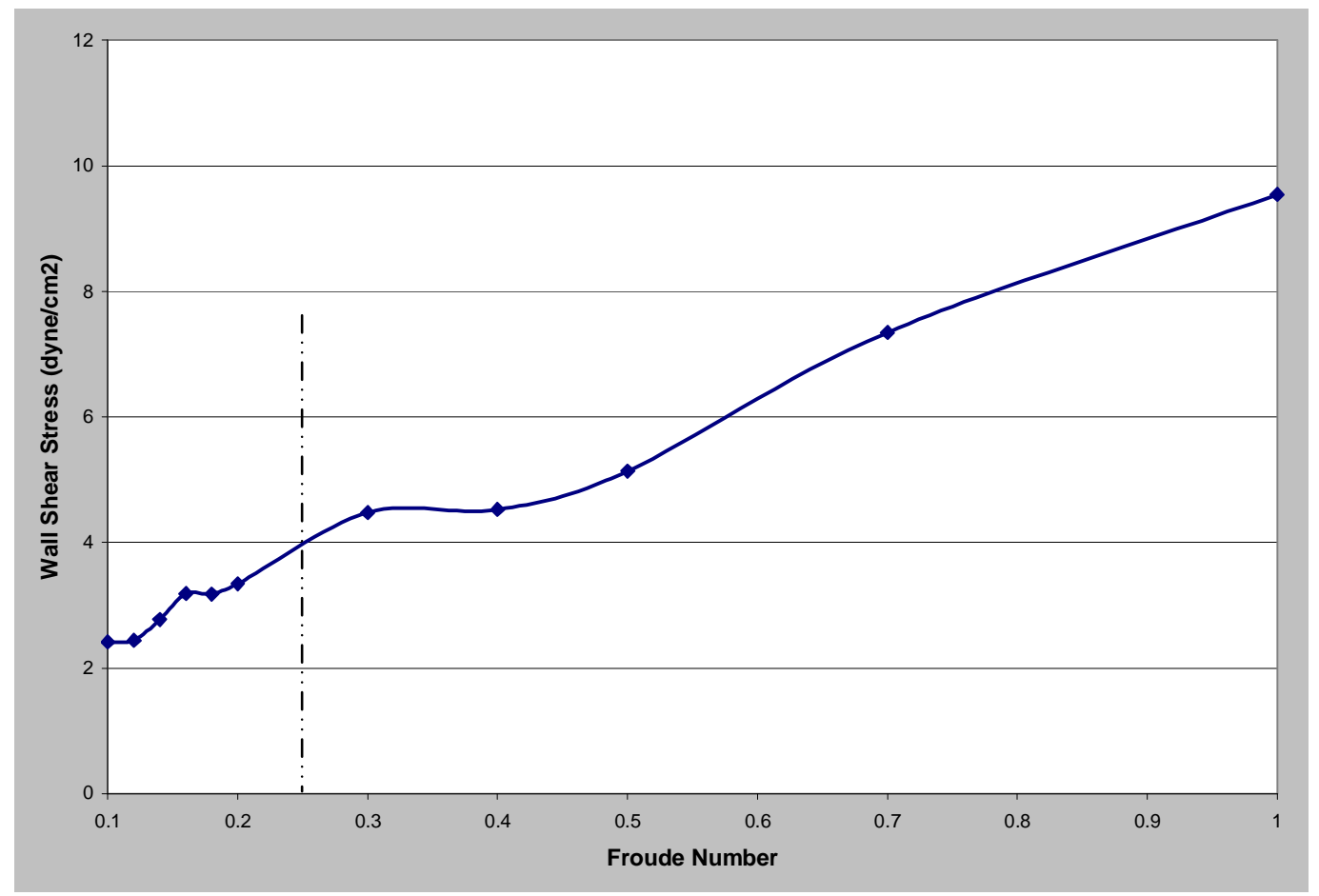

FIGURE 55 - Maximum Bottom Shear Stress versus Froude Number

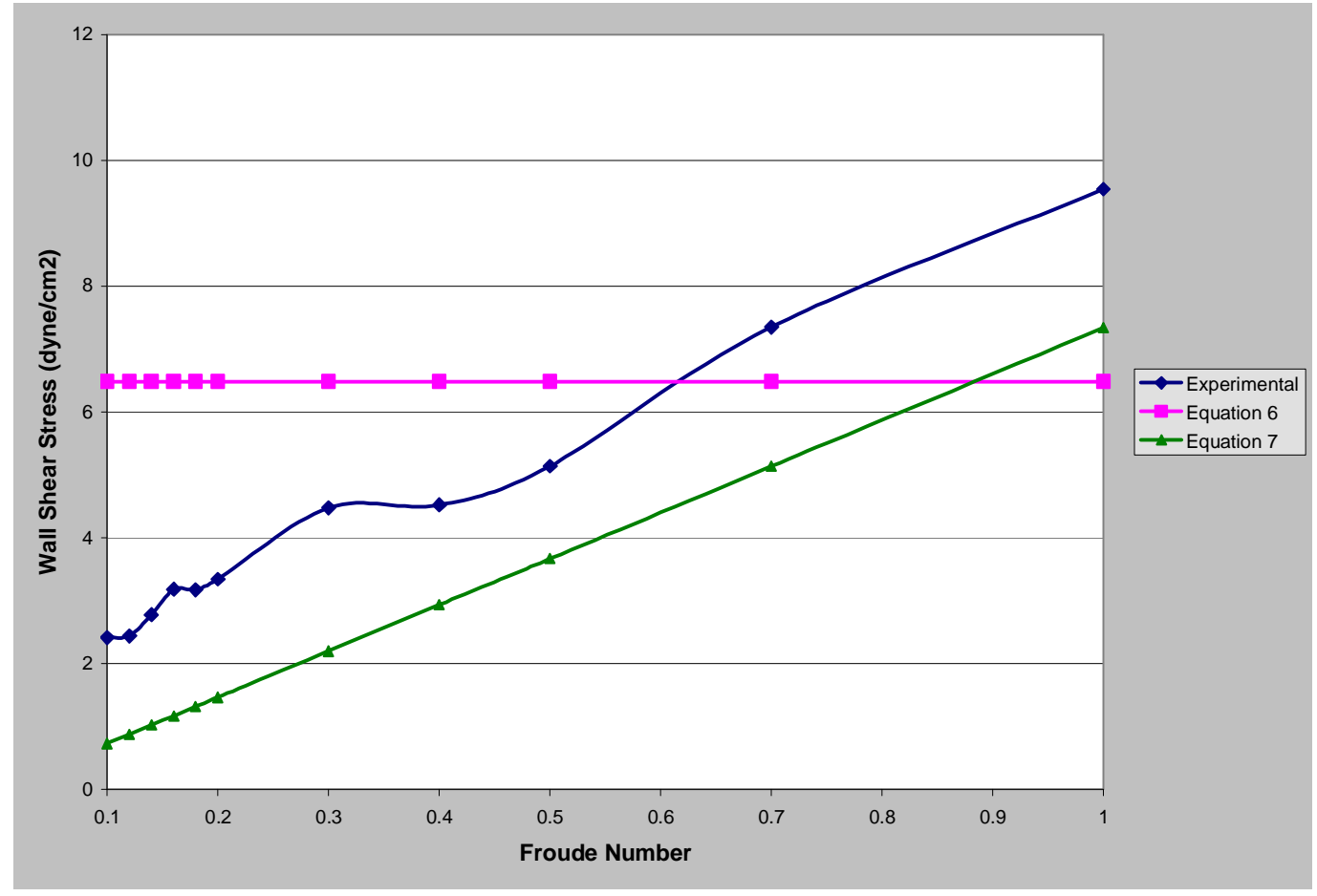

FIGURE 56 - Froude Number Data Comparison to Equations 
Since the effects of maximum WSS have been investigated for several Froude Numbers, the shape of the actual contours will be analyzed to determine what trends exist. Shear contours in the low Froude range from 0.1 to 0.2 are similar as seen in Figures 57-62. Each shows a triangular area of high WSS with a flat edge at the leading edge of the wave near the outer edge of the cylinder. As the Froude Number is increased, the region becomes narrower and the comet tail decreases in length. Additionally, a second area of medium WSS is visible under the trailing edge of the wave. As the Froude Number is increased, the size and relative intensity of this region decreases, developing from a triangular shape to an egg-like shape. After the Froude transition at 0.3, seen in Figure 63, the region of high WSS continues to become narrower, while the comet tail begins to increase in length. Also, a clear definition develops between the region of high WSS and the region of medium WSS. This well defined line of low shear between the two regions was previously seen in Figure 45 where the Stokes cases transitioned from low Froude to high Froude, so it can be definitively stated that this effect in the shear contour is an indication that the case is near, but just past the Froude transition. The Froude Numbers of 0.4 and 0.5, seen in Figures 64 and 65, show that the region of high WSS near the edge of the cylinder has begun to become irregular near the leading edge while the comet tail continues to stretch further around the dish. The area of medium WSS begins to move closer to the area of high WSS, as occurred with the Stokes cases. For the cases of 0.7 and 1.0, seen in Figure 66 and 67, the irregular leading edge of the high WSS region gives way to a thinner, more rounded area with increasingly longer tails. The area of medium WSS continues to move closer to the area of high WSS, essentially connecting at a Froude Number of 1.0. 


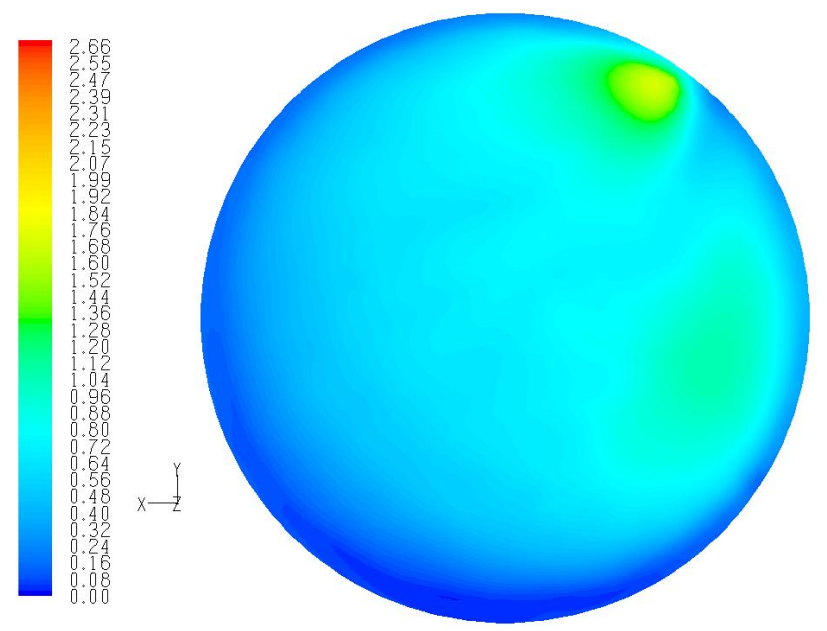

FIGURE 57 - Froude Number 0.1 Wall Shear Stress Contour

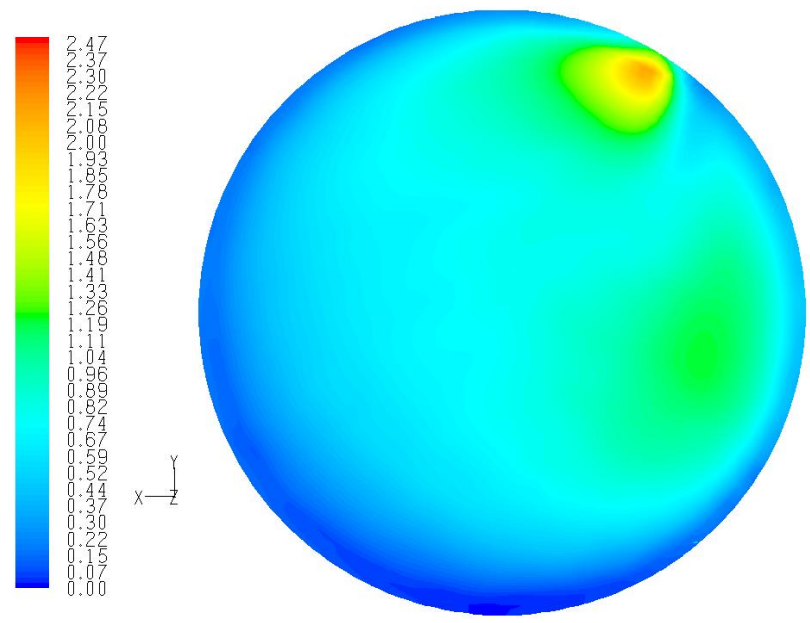

FIGURE 58 - Froude Number 0.12 Wall Shear Stress Contour

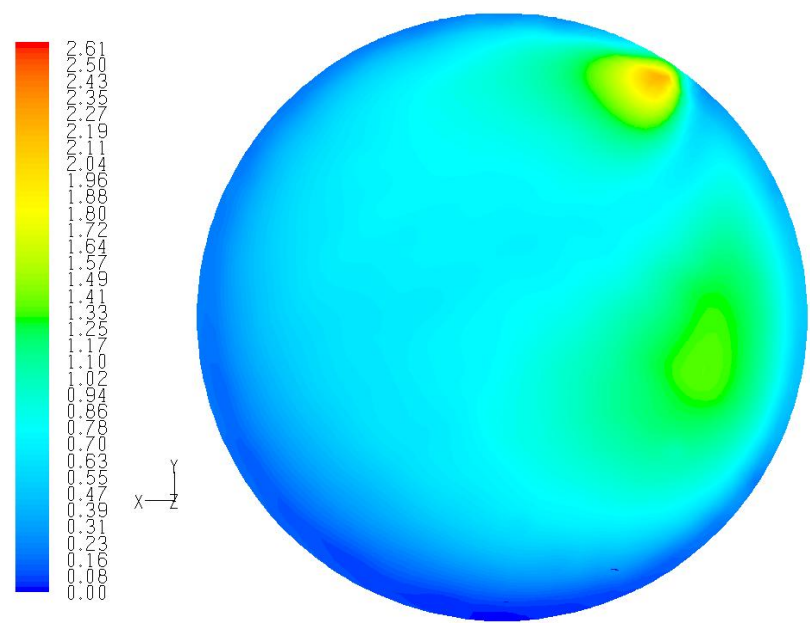

FIGURE 59 - Froude Number 0.14 Wall Shear Stress Contour 


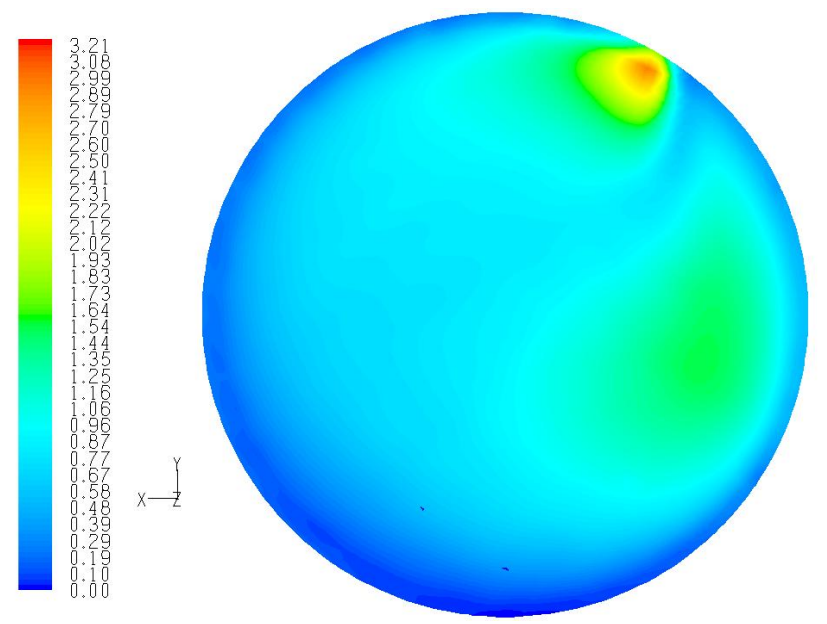

FIGURE 60 - Froude Number 0.16 Wall Shear Stress Contour
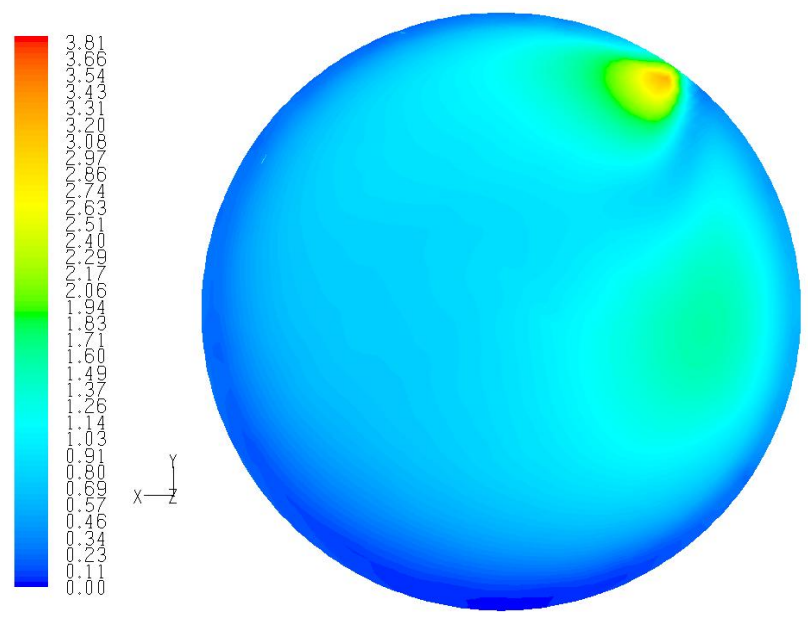

FIGURE 61 - Froude Number 0.18 Wall Shear Stress Contour
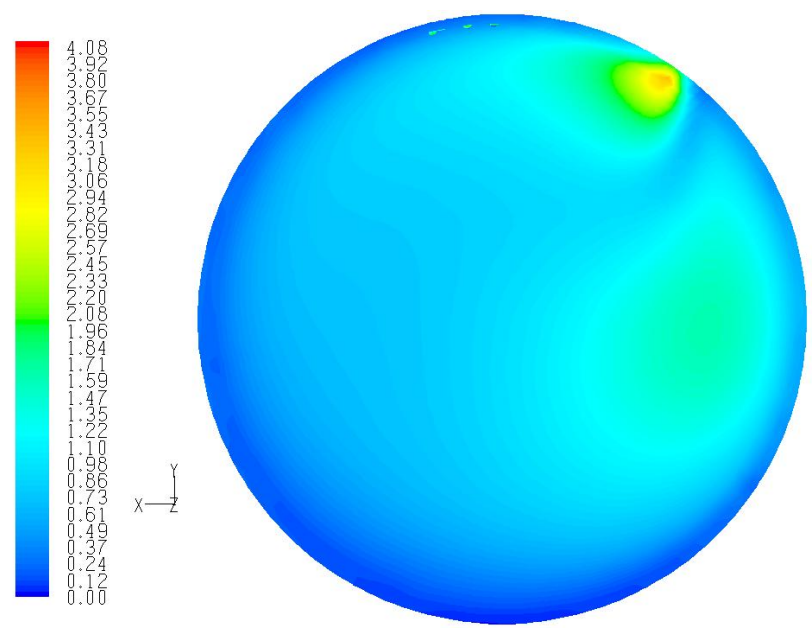

FIGURE 62 - Froude Number 0.2 Wall Shear Stress Contour 


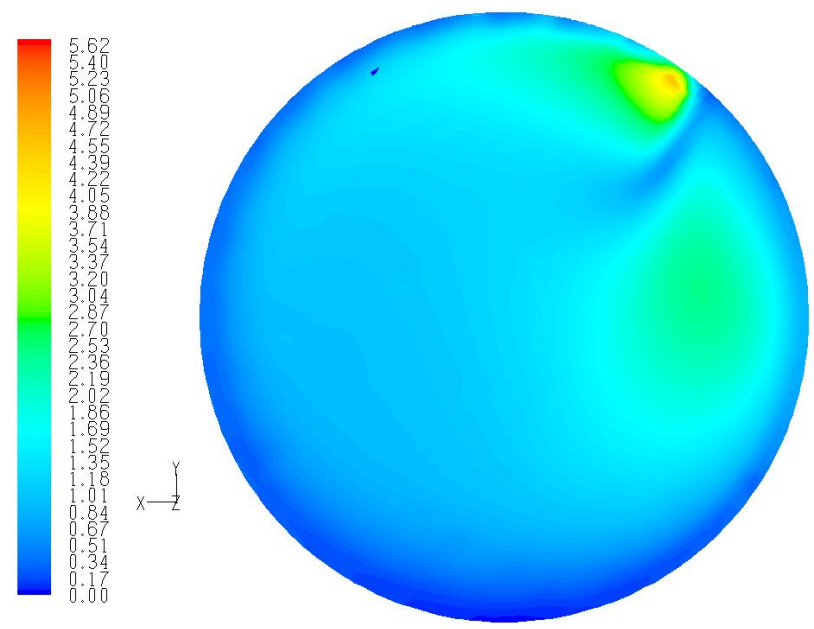

FIGURE 63 - Froude Number 0.3 Wall Shear Stress Contour

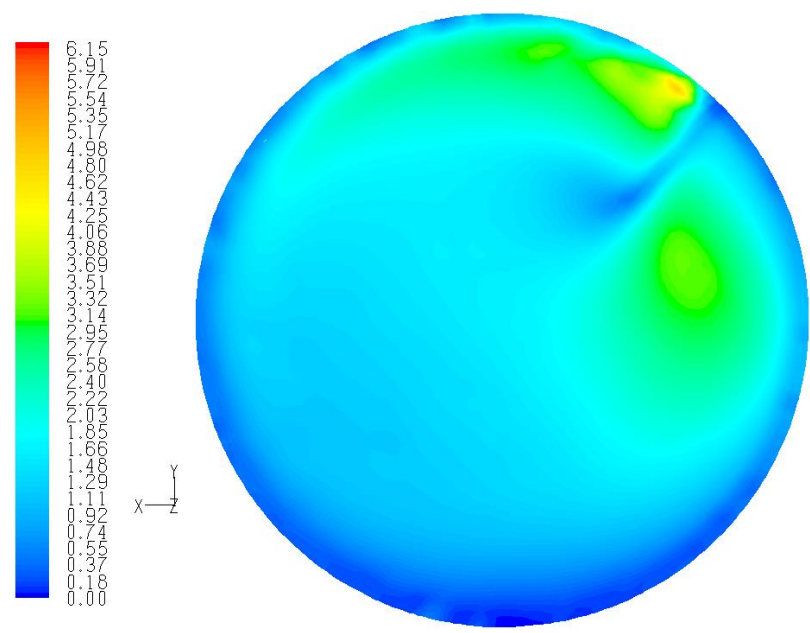

FIGURE 64 - Froude Number 0.4 Wall Shear Stress Contour

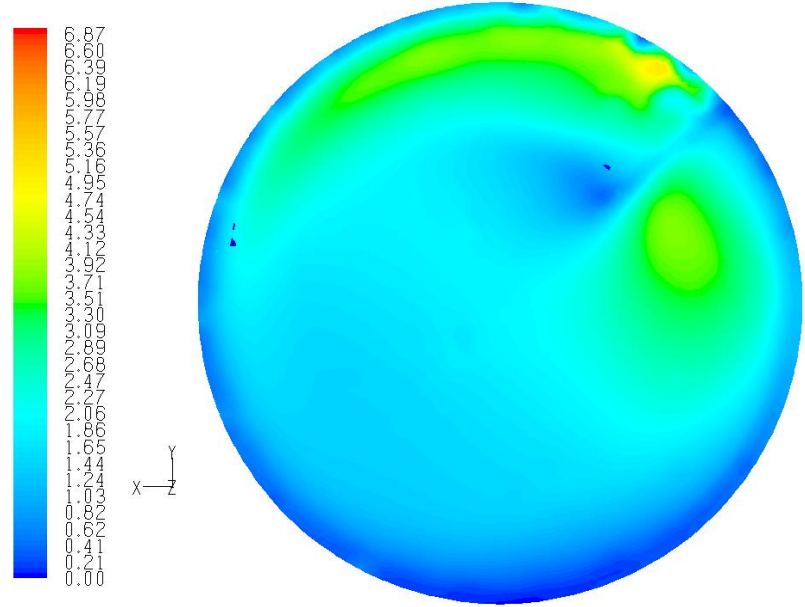

FIGURE 65 - Froude Number 0.5 Wall Shear Stress Contour 


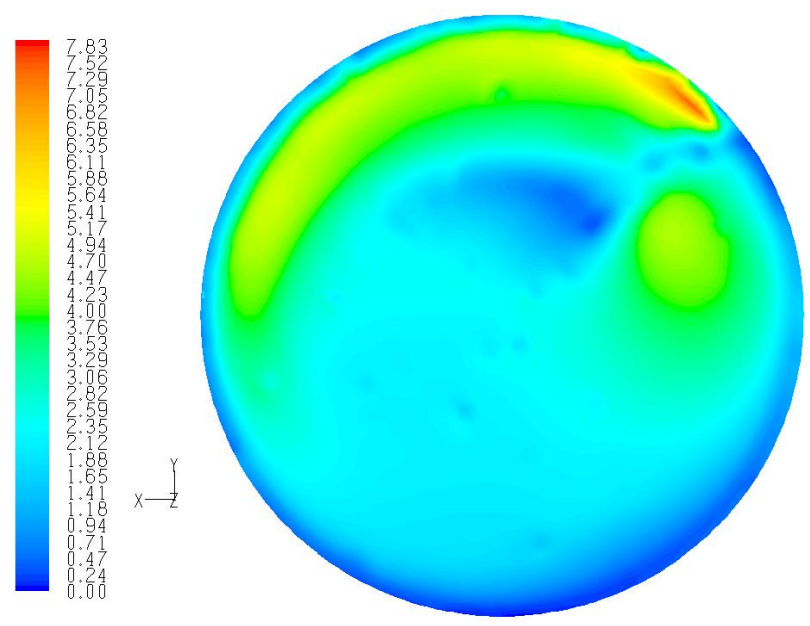

FIGURE 66 - Froude Number 0.7 Wall Shear Stress Contour
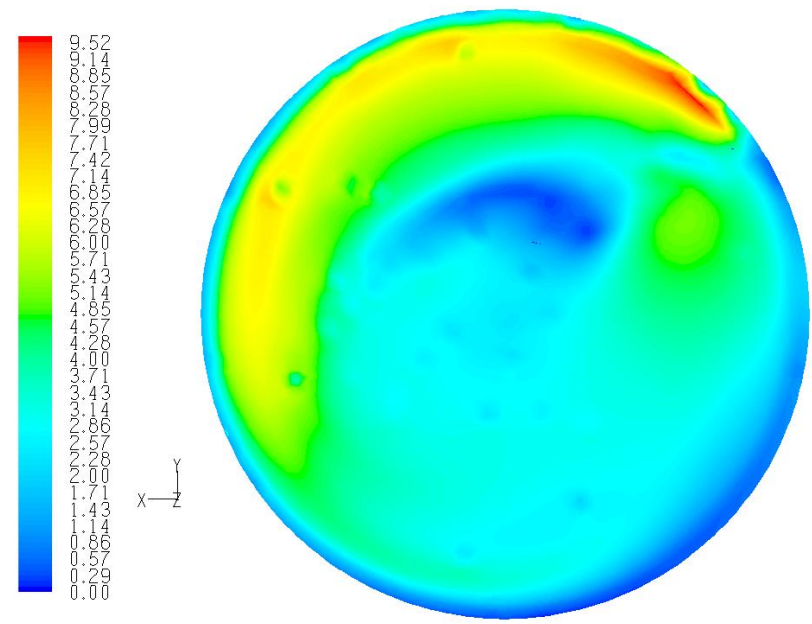

FIGURE 67 - Froude Number 1.0 Wall Shear Stress Contour

\section{Wall Shear Stress Contours as a Function of Slope Ratio}

The first item studied in the investigation of WSS contours as Slope Ratio is varied is the maximum bottom WSS. Values for maximum bottom WSS as the Slope Ratio is varied are shown in TABLE VII for a Stokes Number of 5.0 and a Froude Number of 1.0. Maximum bottom WSS values increase as Slope Ratio is increased ranging from $1.32 \frac{d y n e s}{\mathrm{~cm}^{2}}$ at a Slope Ratio of 0.1 to $16.28 \frac{d y n e s}{\mathrm{~cm}^{2}}$ at a Slope Ratio of 2. 


\section{TABLE VII}

MAXIMUM BOTTOM WALL SHEAR STRESS VS. SLOPE RATIOS

\begin{tabular}{|c|c|}
\hline Slope Ratio & Wall Shear Stress \\
\hline 0.1 & 1.32 \\
\hline 0.2 & 1.90 \\
\hline 0.5 & 7.74 \\
\hline 0.8 & 9.72 \\
\hline 1.1 & 11.36 \\
\hline 1.4 & 15.64 \\
\hline 1.7 & 15.87 \\
\hline 2 & 16.28 \\
\hline
\end{tabular}

To study how the Slope Ratio transition affects the maximum bottom WSS, a plot was generated in Figure 68. The Slope transition occurs between 1.1 and 1.4; a large change in WSS is observed at the transition. Above the transition, WSS values level off. Although from the graph it looks as if WSS decreases at 1.7, this is only an inaccuracy in Excel's smooth line plotting system as Table VII clearly shows the value is increasing. The graph shows a linear regression of WSS below the transition with the exception of the lowest Slope Ratio values at 0.1 and 0.2 . As previously shown, these two cases do not have a well developed leading edge. The leading edge of the wave produces the greatest WSS values on the bottom of the cylinder, hence these cases have a low maximum WSS. A comparison of the Slope data collected from FLUENT to Equation (6) and Equation (7) is seen in Figure 69. Equation (6) follows a parabolic trend somewhat similar to that of the data. Equation (6) follows most closely in the low slope region. This is expected as the equation is derived for low Slope ratios. Equation (7) has a flat slope and shows no correlation to the experimental maximum WSS data 


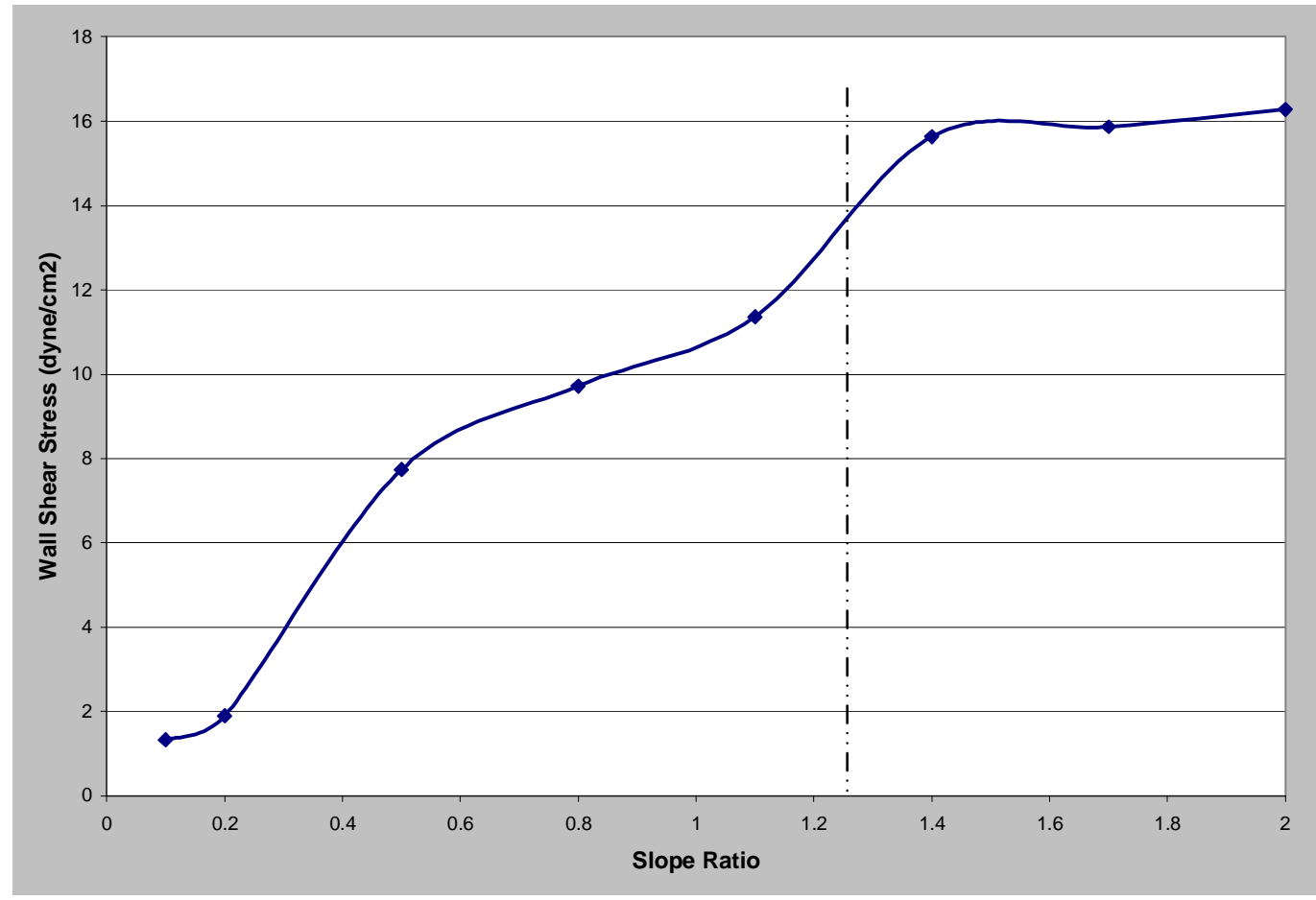

FIGURE 68 - Maximum Bottom Wall Shear Stress versus Slope Ratio

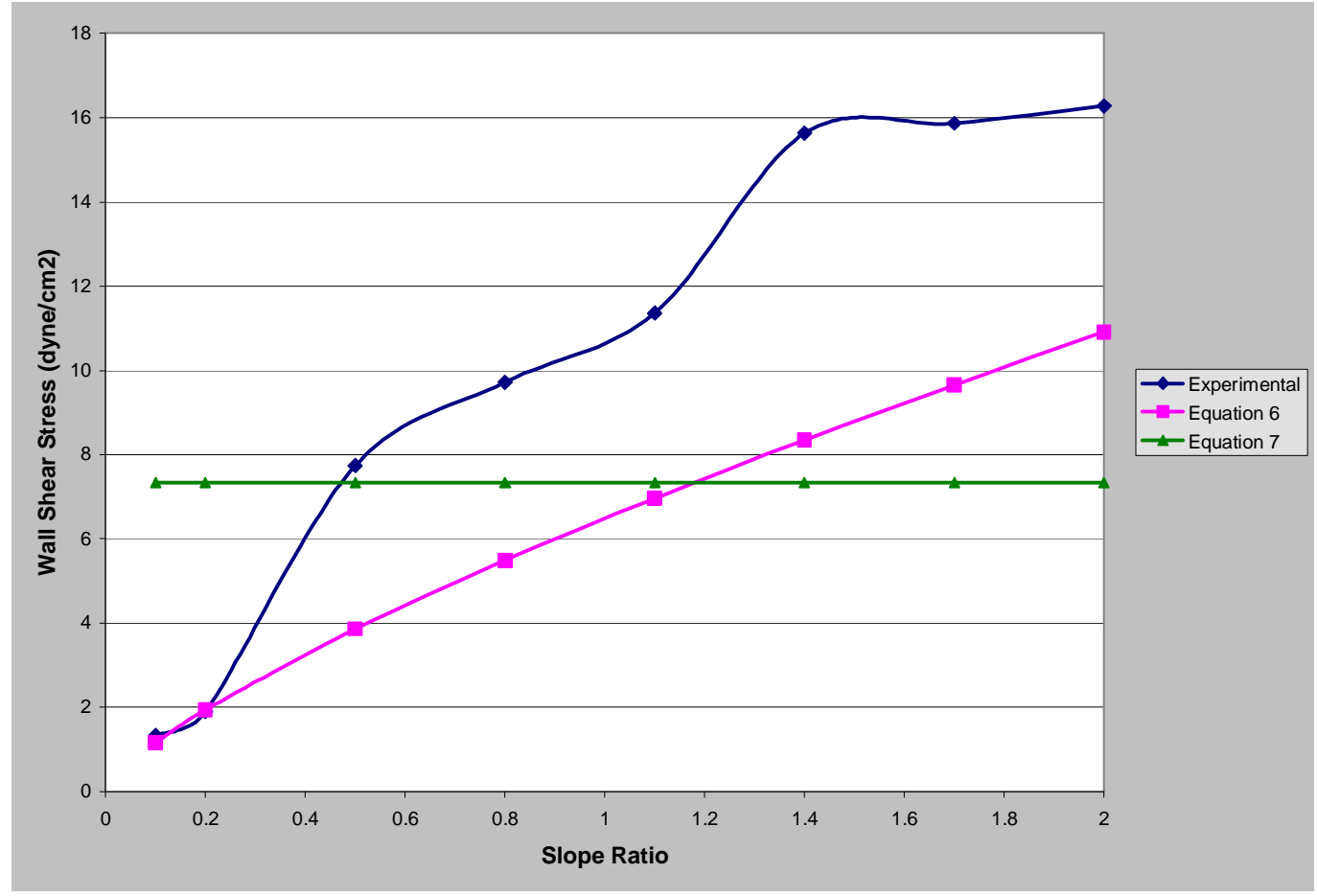

FIGURE 69 - Froude Number Data Comparison to Equations 
Since the effects of maximum WSS have been investigated for several Slope Ratios, the shape of the actual contours will be analyzed to determine what trends exist. WSS Contours for the Slope Ratios of 0.1 and 0.2 depart from those normally viewed as seen in Figures 70 and 71. Each of the four areas of high WSS on these two cases result from one of the four peaks, with the higher WSS values occurring at the taller peaks. At the Slope Ratio of 0.5, seen in Figure 72, a thin region of high WSS develops at the leading edge near the perimeter of the cylinder. In the long tail that follows, a second region of high WSS is present, although not as high in magnitude as the region at the leading edge. For the Slope Ratio of 0.8, as seen in Figure 73, the area of high WSS becomes exceedingly large. Normally the maximum WSS occurs just behind the leading edge and then WSS slowly decreases through the tail. In this case, the region of high WSS extends more than $40 \%$ around the perimeter of the cylinder. The region of medium high WSS observable in many cases is just visible at the end of the trailing edge. The Slope Ratio of 1.1, seen in Figure 74, shows a small, long thin area of high WSS at the leading edge of the wave with a long tail of tapering WSS values. This case shows a fading straight line of low WSS between the area of high WSS and the area of medium high WSS. This signifies that this case is just past the Froude transition. These phase contours that show this Froude transition are seen in Figures 34 and 35. After the Slope transition, the WSS contours for the Slope Ratios of 1.4, 1.7, and 2.0, seen in Figures 7577, look remarkably similar, which explains why the maximum WSS values tapered off. Each case has a small, round area of high WSS at the leading edge with a long tail whose values taper off quickly. The main difference between the three cases is that as the Slope Ratio is increased, the area of medium high WSS at the trailing edge drifts further from 
the leading edge. This is because as the Slope Ratio is increased the dry area between the leading edge and trailing edge increases, effectively shifting the trailing edge.
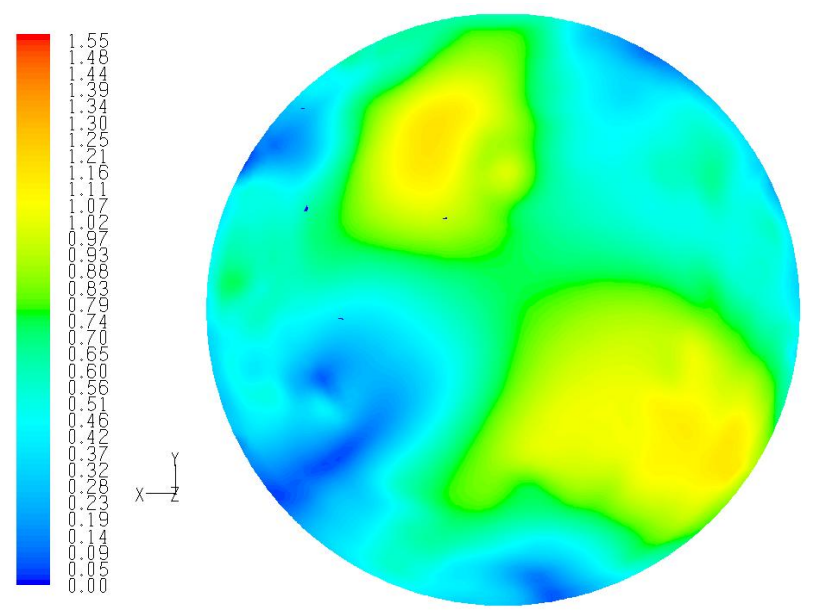

FIGURE 70 - Slope Ratio 0.1 Wall Shear Stress Contour
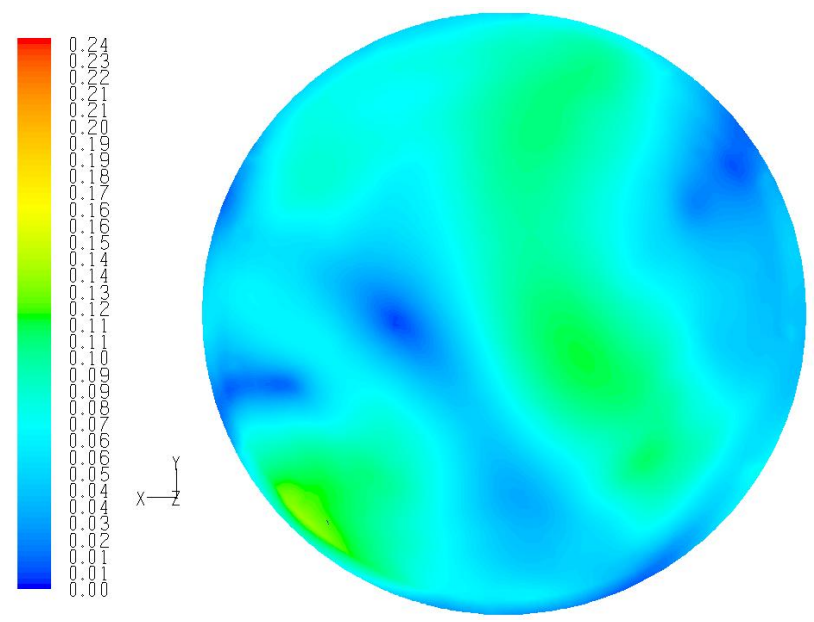

FIGURE 71 - Slope Ratio 0.2 Wall Shear Stress Contour 

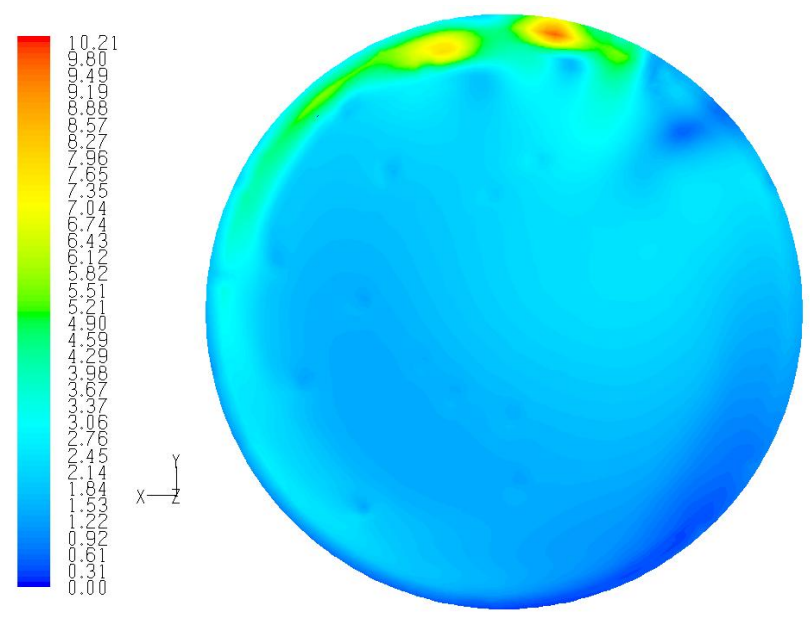

FIGURE 72 - Slope Ratio 0.5 Wall Shear Stress Contour
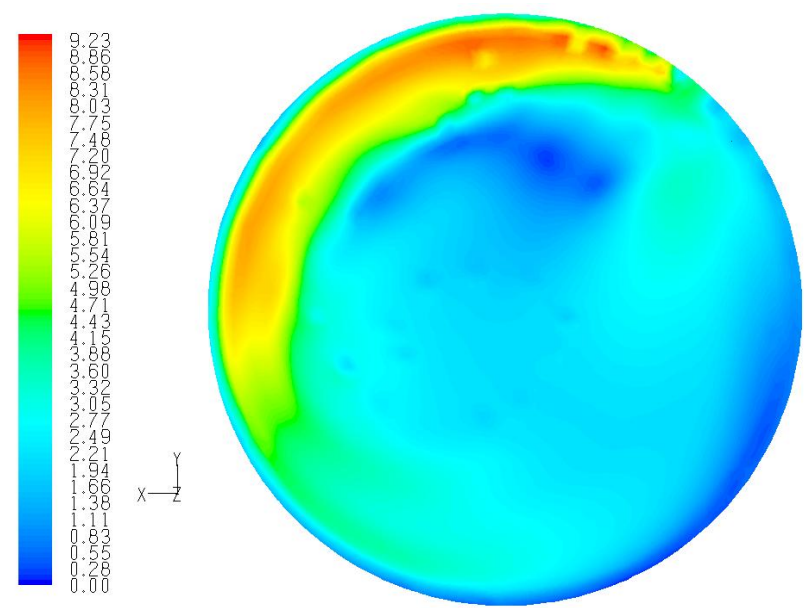

FIGURE 73 - Slope Ratio 0.8 Wall Shear Stress Contour
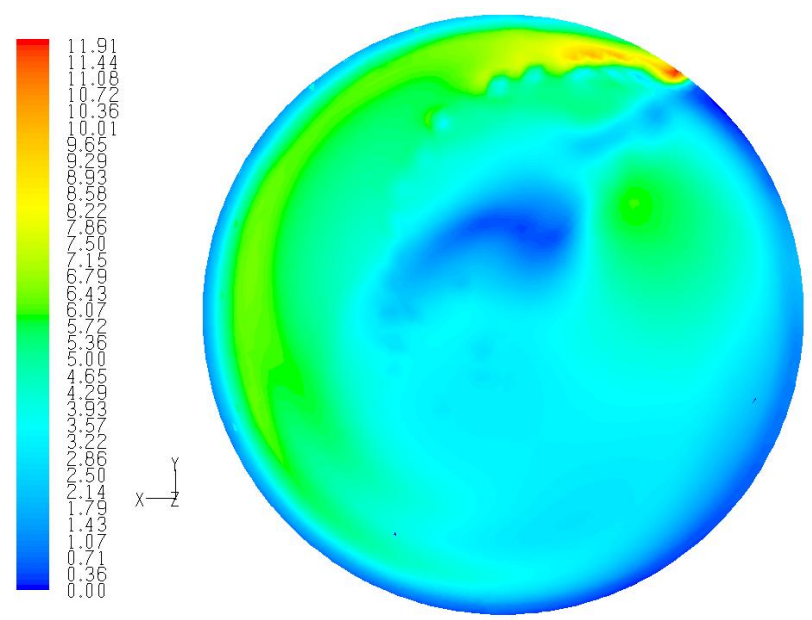

FIGURE 74 - Slope Ratio 1.1 Wall Shear Stress Contour 

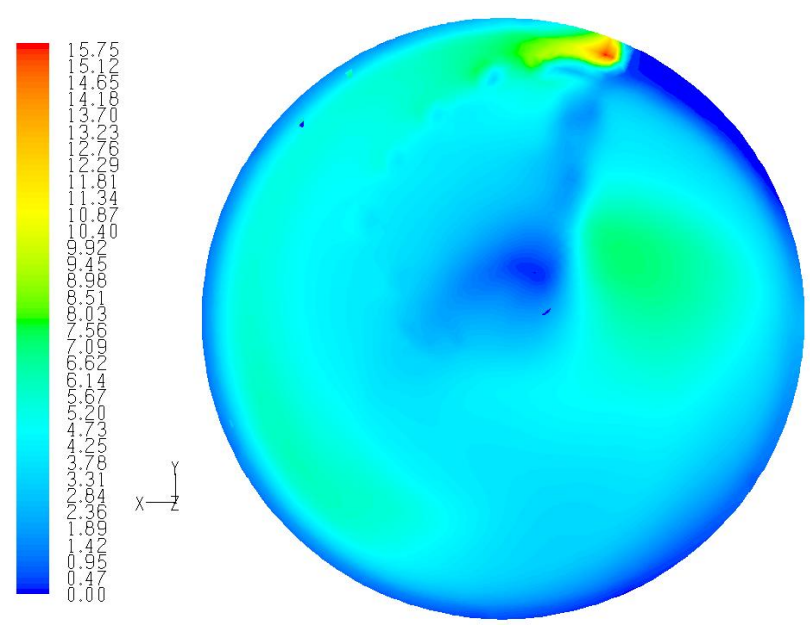

FIGURE 75 - Slope Ratio 1.4 Wall Shear Stress Contour
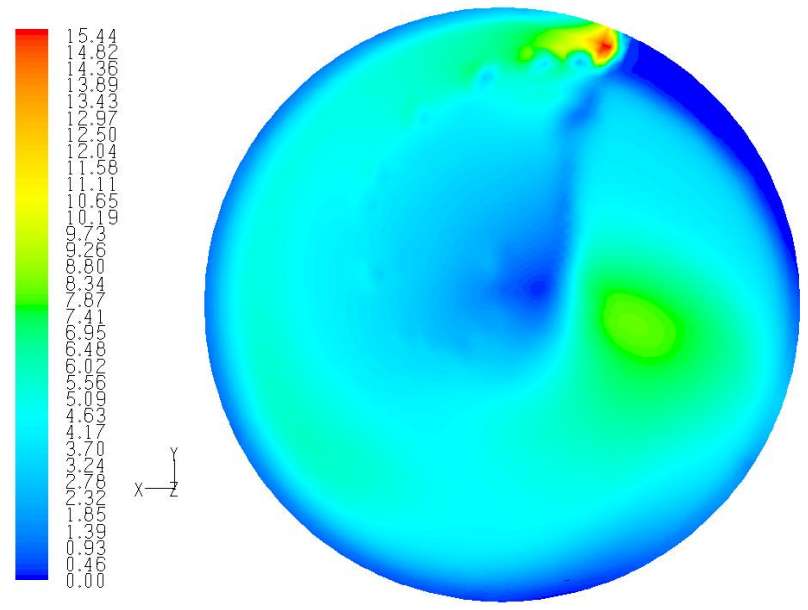

FIGURE 76 - Slope Ratio 1.7 Wall Shear Stress Contour
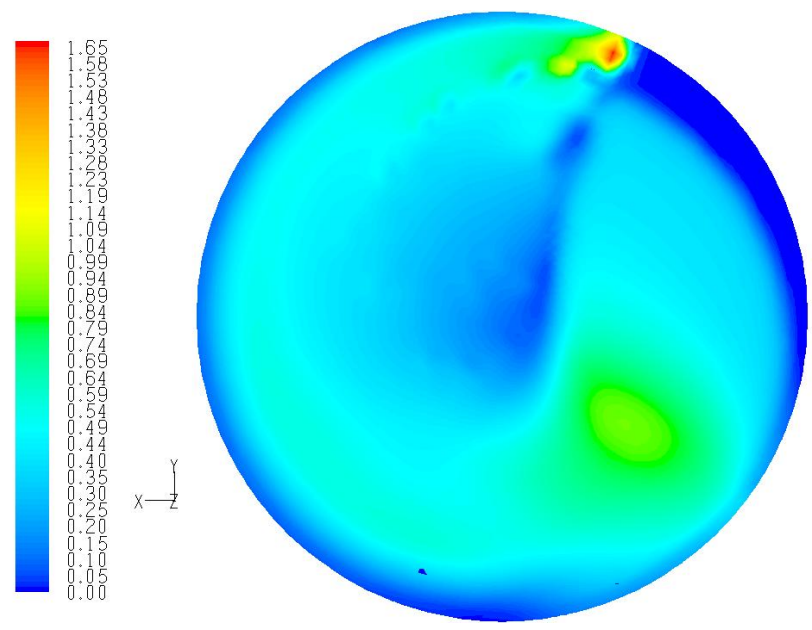

FIGURE 77 - Slope Ratio 2.0 Wall Shear Stress Contour 


\section{Analysis of Plots of $X$ and Y Wall Shear Stress Components}

\section{Construction of Plots of $X$ and $Y$ Wall Shear Stress Components}

Plots of X and Y WSS components can be created to graphically show the changes in WSS at a set radius. Plots of X and Y WSS components are created from the $\mathrm{X}$ and Y WSS contours. When values from the X WSS contour are coupled with values from the same location on the Y WSS contour, it creates points that should comprise an ellipse. The four radii of study for the elliptical plots are $20 \%, 40 \%, 60 \%$, and $80 \%$ of the maximum radius. For $20 \%$ and $40 \%$, values are extracted from circular grids of 32 equally spaced points on each contour while for $60 \%$ and $80 \%$, circular grids of 64 equally spaced points are used. Presented is a plot containing all four radii along with the corresponding $\mathrm{X}$ and $\mathrm{Y}$ WSS contours. If a contour has a single high value region that leads to a low value region then comes back to the high value region when completing the circle around the radius studied, the corresponding plot would be of a perfect ellipse, while if a plot intersects itself, it indicates that that radius crosses through multiple high and low shear value regions. The exact shape of a plot is unimportant compared to how the plot relates to those with similar values. It is from major differences between plots, especially in the 60 and $80 \%$ range as this is where a majority of changes occurs, where effects are noticed.

\section{Plots of $X$ and $Y$ Wall Shear Stress Components as a Function of Stokes Number}

Plots of X and Y WSS components as Stokes Number is varied are analyzed to determine if a visible effect of the Stokes Transition is noticeable and how the plots change throughout the range. A plot of $\mathrm{X}$ and Y WSS components is not possible for the Stokes Number of 1.0 as the WSS values are so low that FLUENT calculates negligible 
values. A plot for the Stokes Number of 2.0 is seen in Figure 78. The values at $40 \%$, $60 \%$, and $80 \%$ cross at this plot. $60 \%$ and $80 \%$ have triangular shapes with crossed circular lobes at the bottom right while $40 \%$ has a figure eight shape and $20 \%$ has an ovoid shape. This plot is substantially different from the plot for the Stokes Number of 3.0 seen in Figure 79. This plot shows a figure eight shape for $20 \%$ and $40 \%$, with $40 \%$ crossing itself in the middle multiple times. $60 \%$ and $80 \%$ have oval shapes with lobes on the far right side pointing upward; the lobe for $60 \%$ crosses. Since these two plots are greatly different, it indicates some major transition occurs. From studying the WSS contours it is known that an effect resembling a Froude transition occurs between these values seen in Figures 44 and 45. The free surface plot for the Stokes Number of 4.0, seen in Figure 80, shows a direct progression of the free surface plot that appeared for the Stokes Number of 3.0. The same shapes are detected but the upward facing lobes on the right side are much larger. As such, the plots of X and Y WSS components show no transition between 3.0 and 4.0. The plot for a value of 4.2, seen in Figure 81, however does have a different shape. While $20 \%$ retains a triangular shape, $40 \%$ forms an uncrossing triangle. $60 \%$ forms an oval with a protrusion at the bottom right and $80 \%$ forms an oval with a small right facing protrusion. The difference between 4.0 and 4.2 is not as substantial as between 2.0 and 3.0, but it is enough to state that a transition occurs; in this case the Stokes transition. A small change is acceptable for the Stokes transition as it has the smallest physical effect on the wave of the three dimensionless parameters. The plots of X and Y WSS components for the Stokes Numbers of 4.4 through 5.0, seen in Figures 82-85, are direct progressions of the plot in 4.2 signifying that no transition occurs in this region. The plots for $20 \%$ remain figure eight shaped throughout the range. 
While the exact orientation varies slightly, $40 \%$ retains a roughly triangular shape. The plot for $60 \%$ becomes wider, with the protrusion becoming more pronounced as Stokes Number is increased. The plot for the Stokes number of 10, seen in Figure 86, is substantially different than that of 5.0. The plot for $20 \%$ is almost circular, with $40 \%$, $60 \%$, and $80 \%$ getting progressively wider, creating ovals. $80 \%$ has a lobe that is created by a crossing on the middle bottom. As Froude and Stokes transitions have occurred previously, this difference must be caused by an effect resembling the Slope transition. Visual inspection of the free surface plots for Stokes values of 5.0 and 10 in Figures 87 and 88 shows that no concavity is present at 5.0 while a concavity is present at 10 , agreeing with the conclusion of the elliptical plot. 

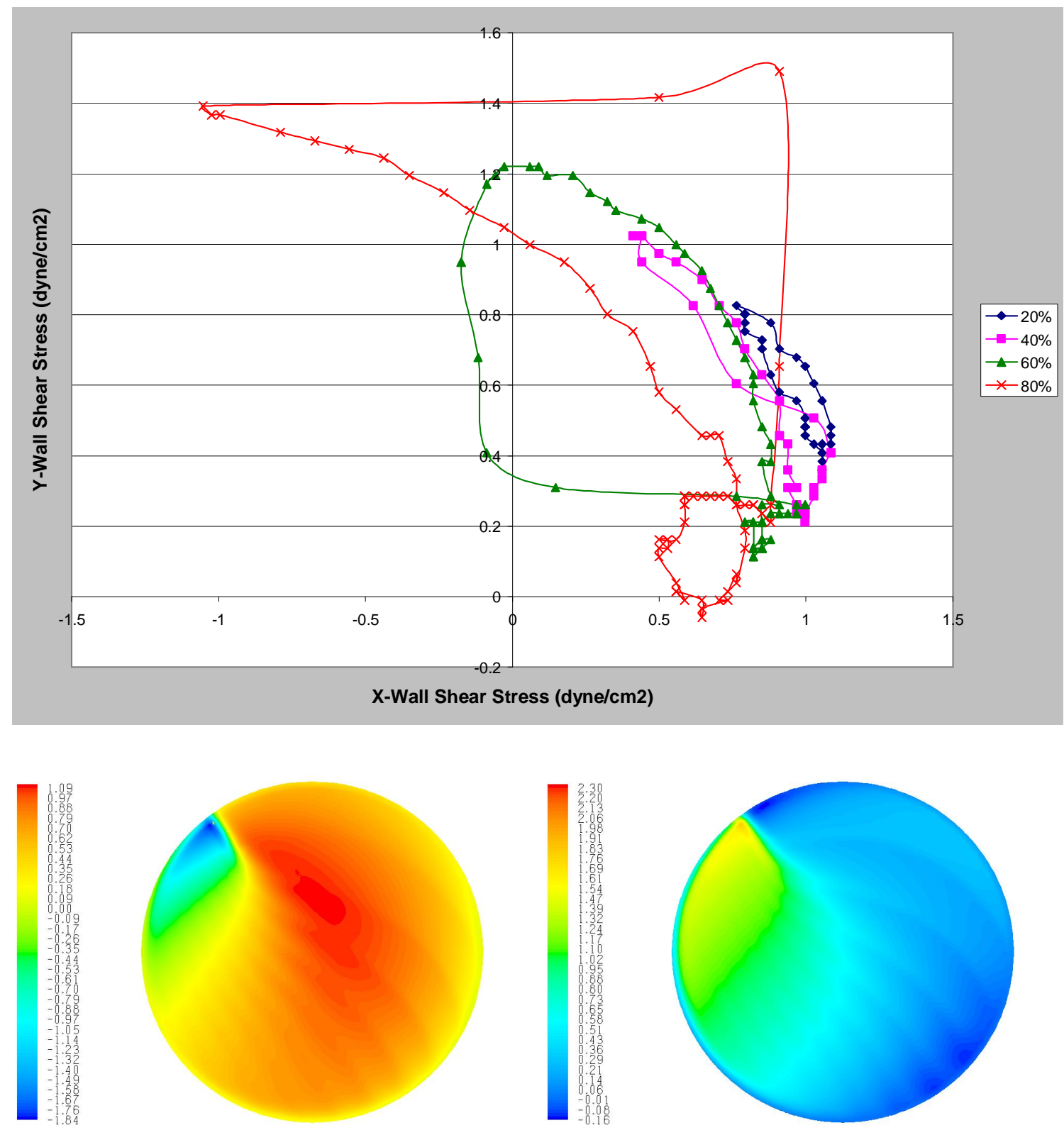

FIGURE 78 - Stokes Number 2.0 Plot of X and Y WSS Components and X and Y WSS

Contours 

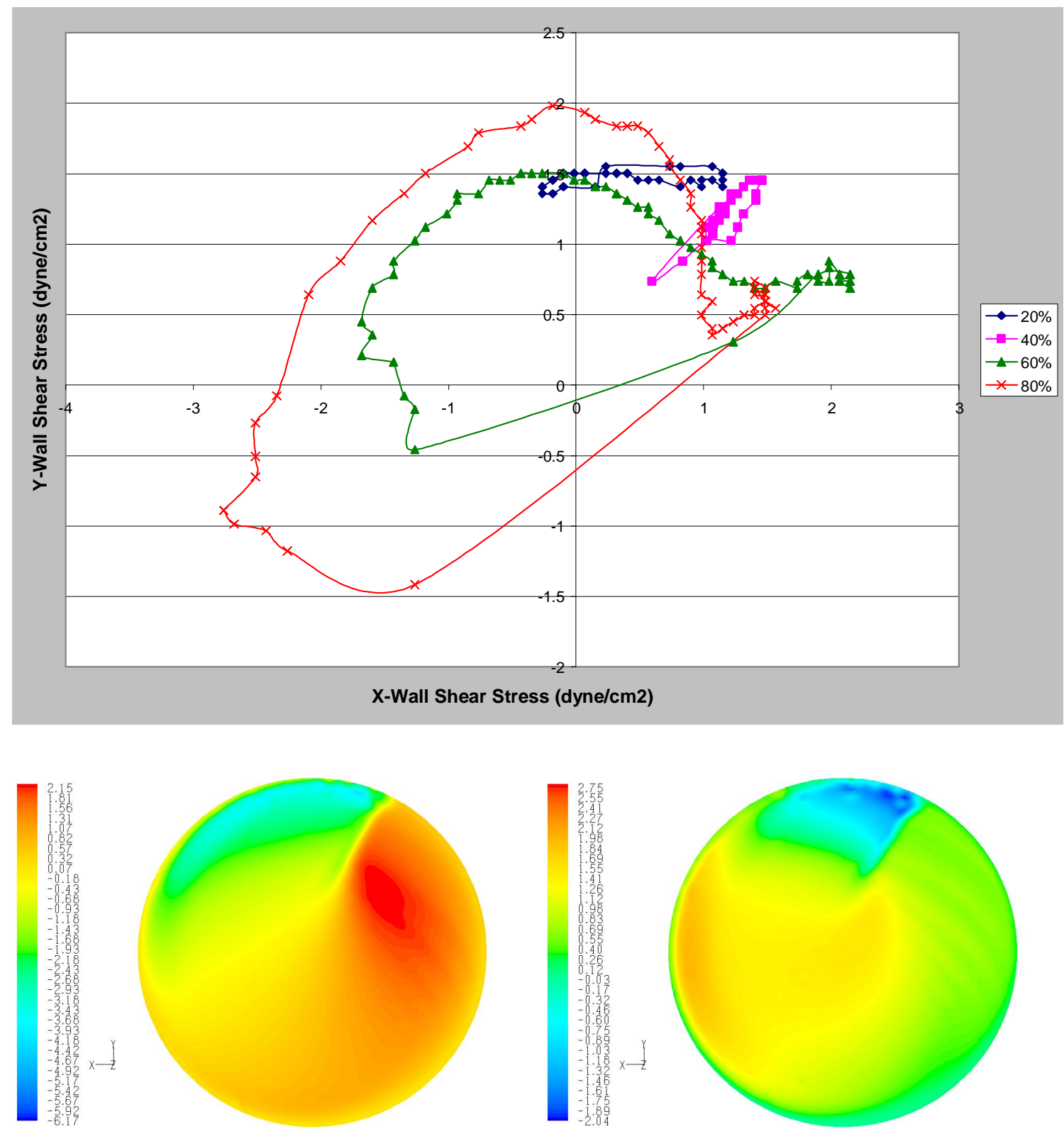

FIGURE 79 - Stokes Number 3.0 Plot of X and Y WSS Components and X and Y WSS

Contours 

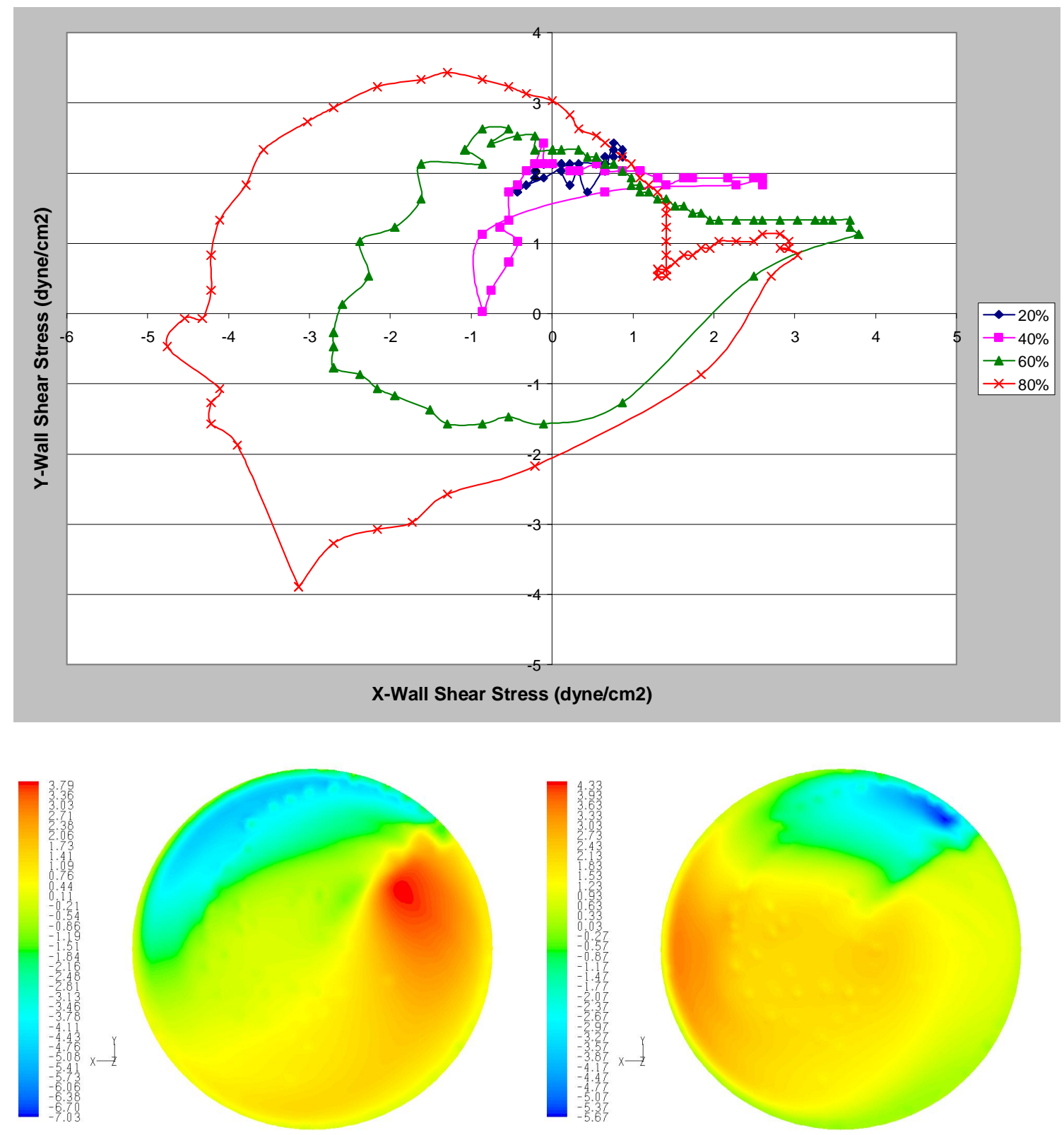

FIGURE 80 - Stokes Number 4.0 Plot of X and Y WSS Components and X and Y WSS

Contours 

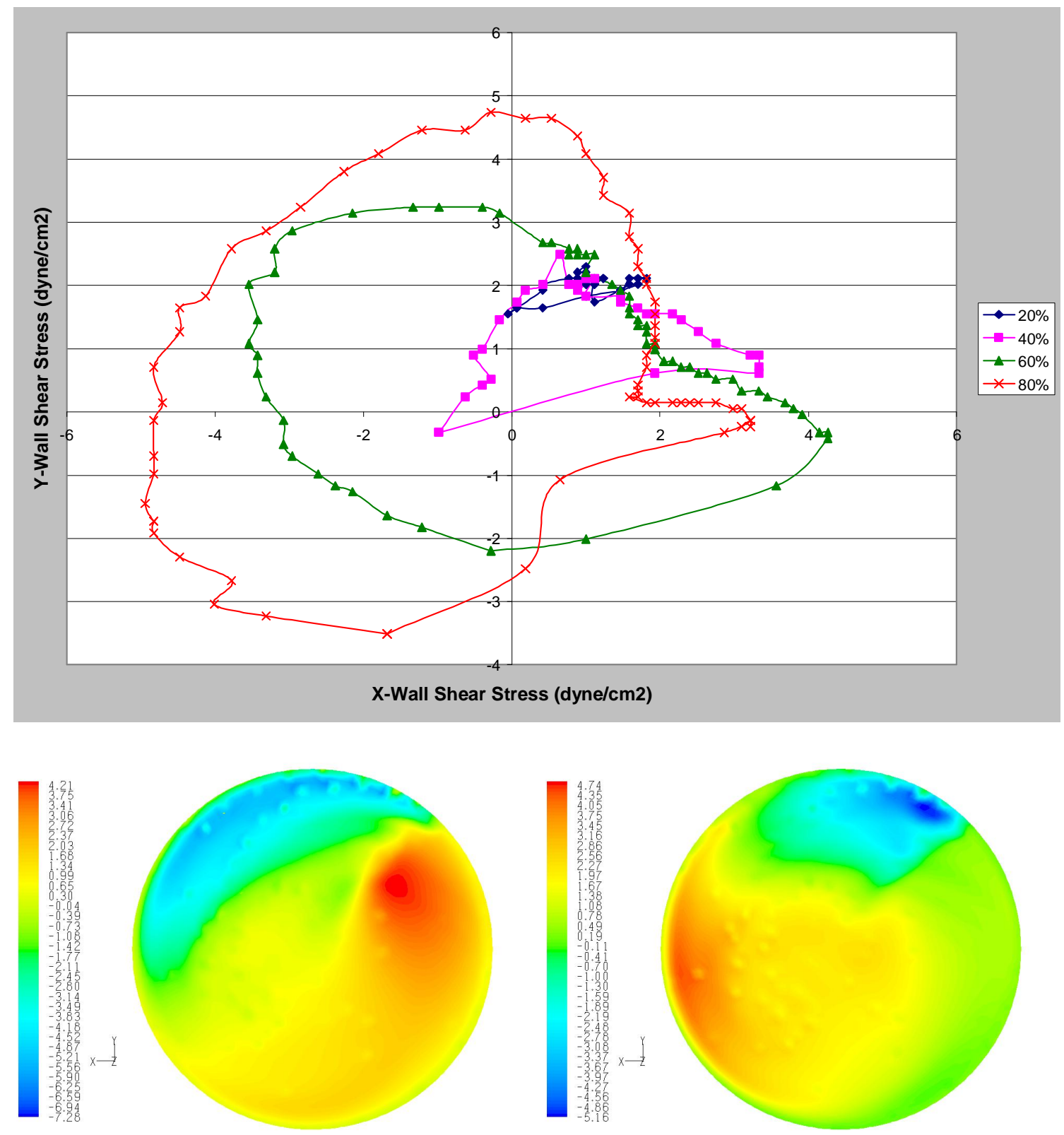

FIGURE 81 - Stokes Number 4.2 Plot of X and Y WSS Components and X and Y WSS

Contours 

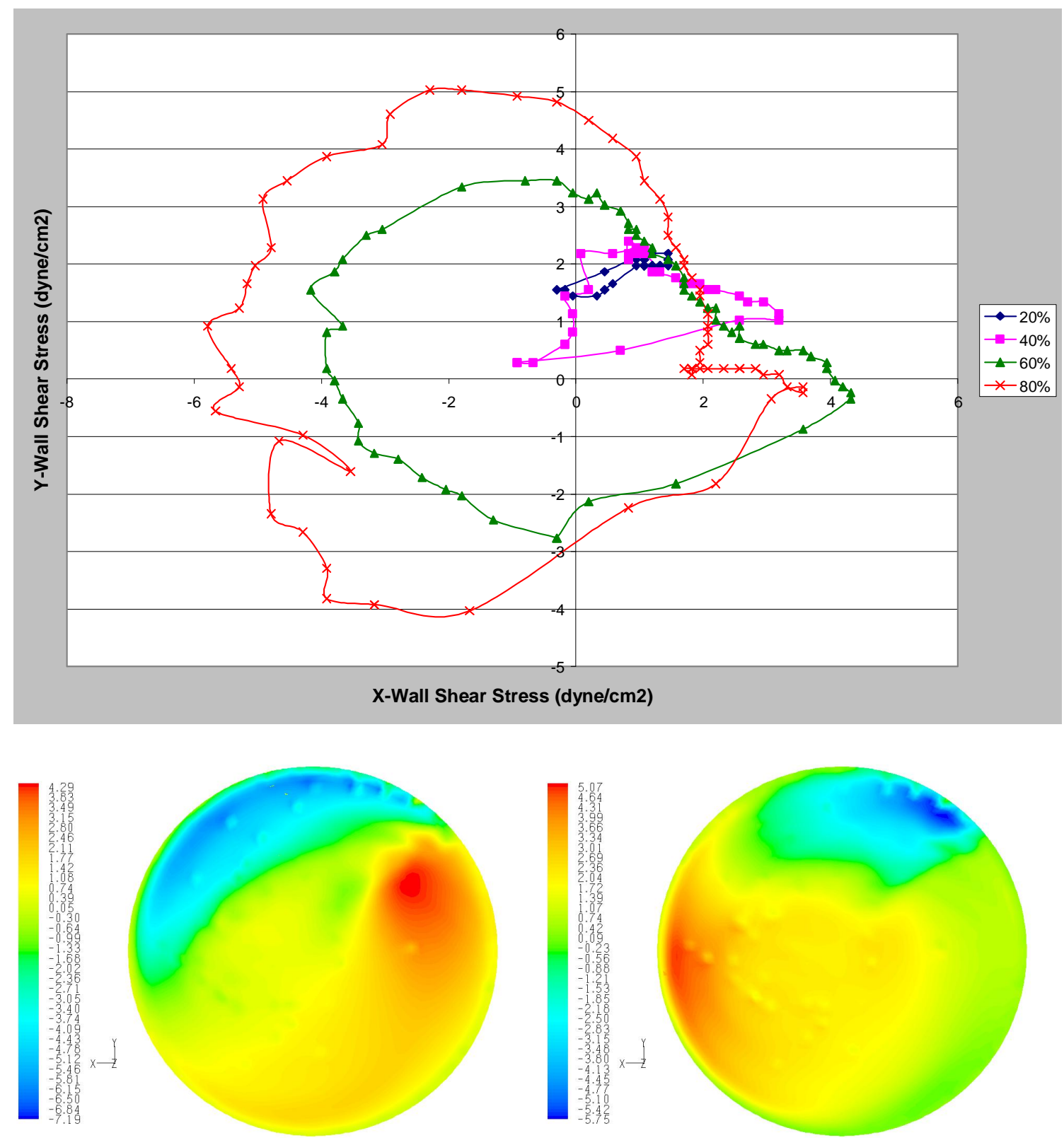

FIGURE 82 - Stokes Number 4.4 Plot of X and Y WSS Components and X and Y WSS

Contours 

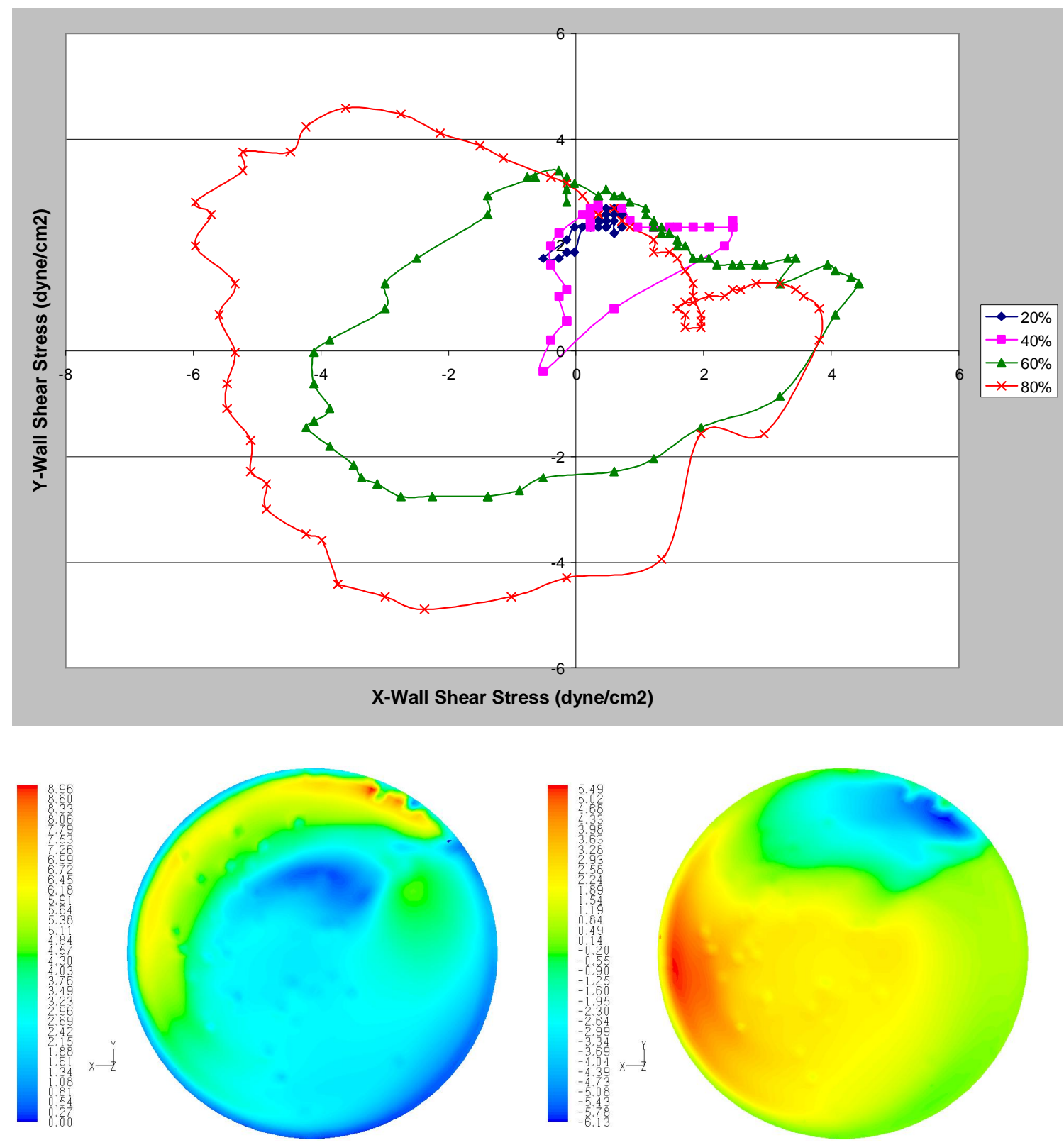

FIGURE 83 - Stokes Number 4.6 Plot of X and Y WSS Components and X and Y WSS

Contours 

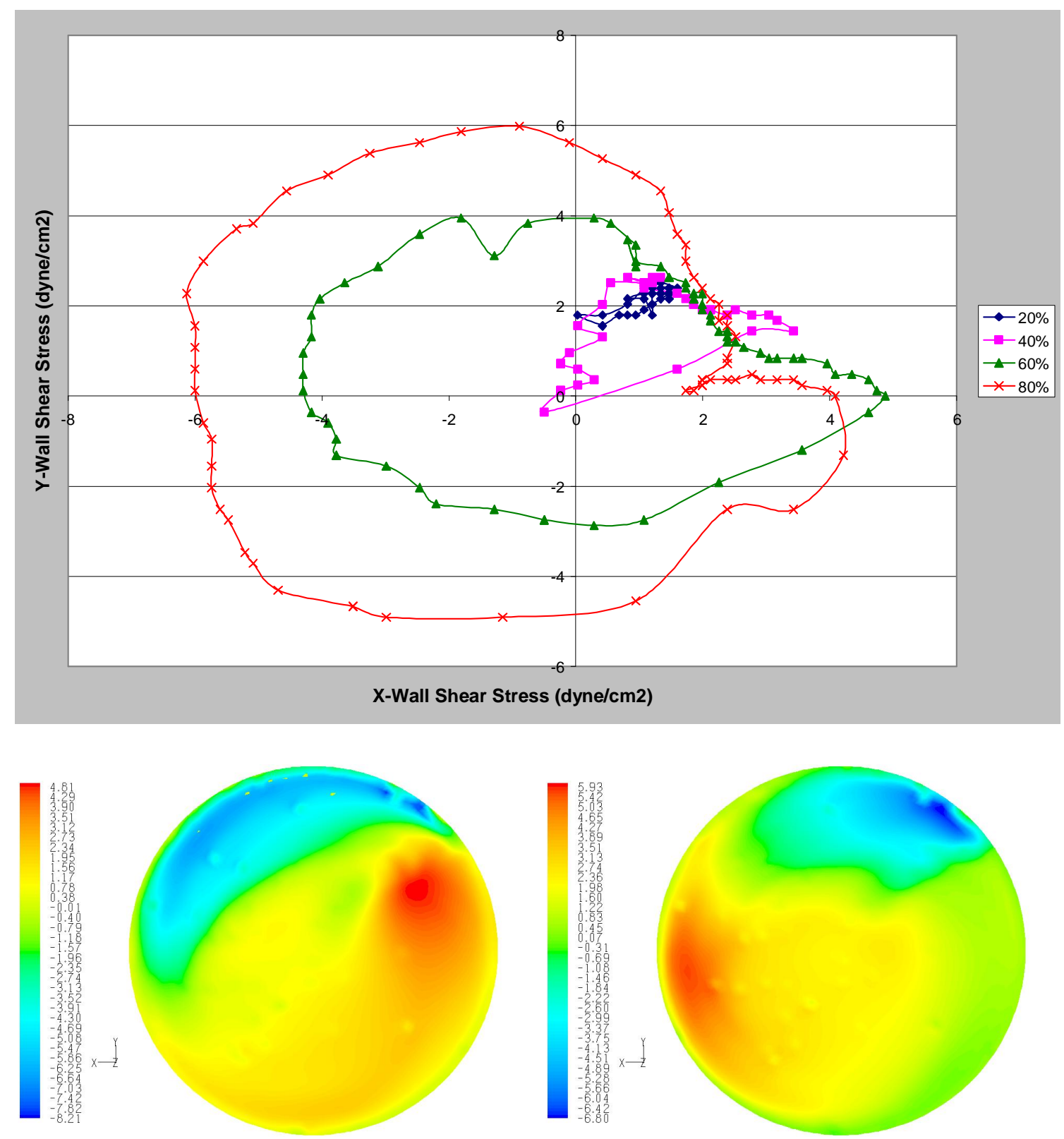

FIGURE 84 - Stokes Number 4.8 Plot of X and Y WSS Components and X and Y WSS

Contours 

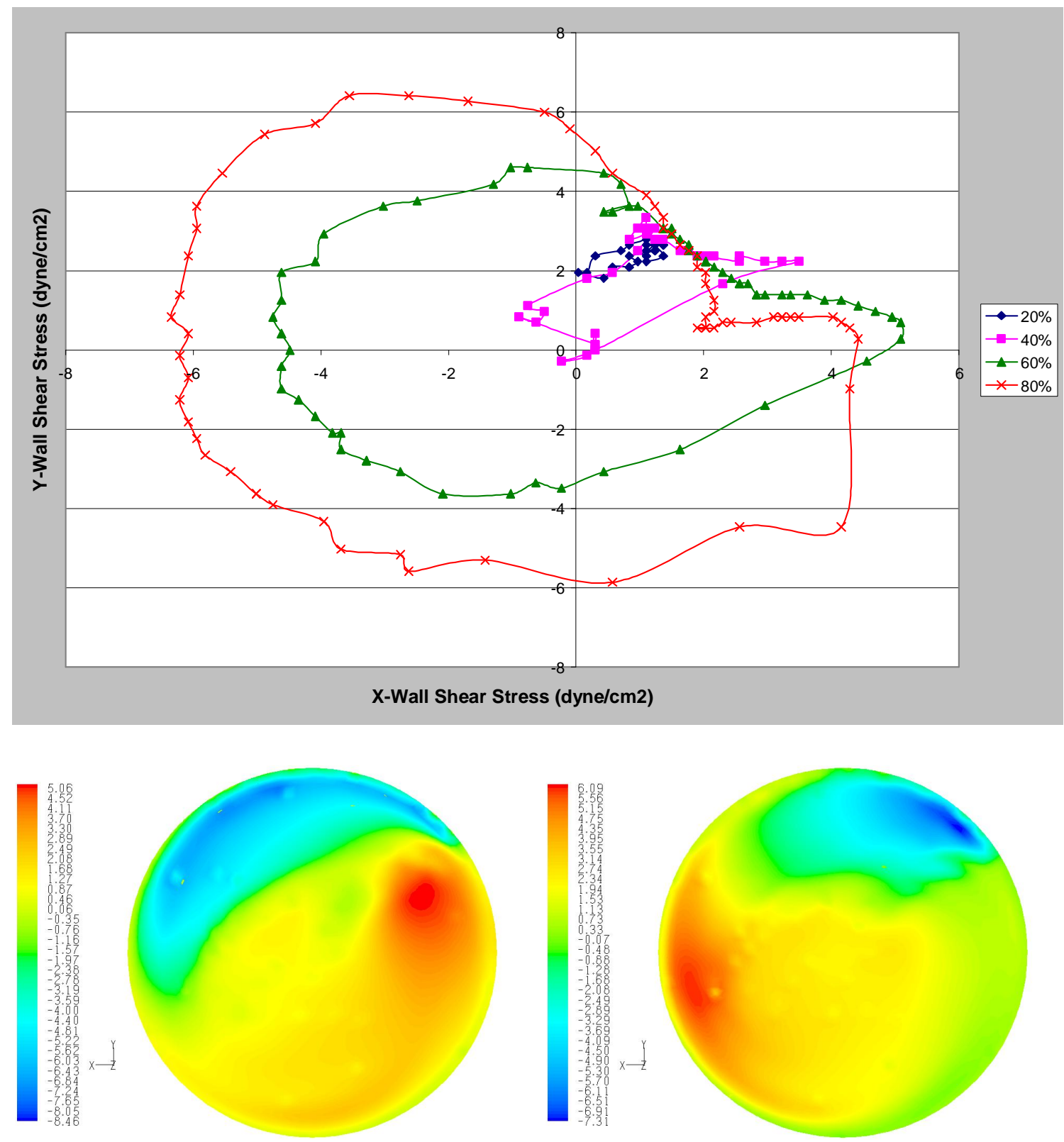

FIGURE 85 - Stokes Number 5.0 Plot of X and Y WSS Components and X and Y WSS

Contours 

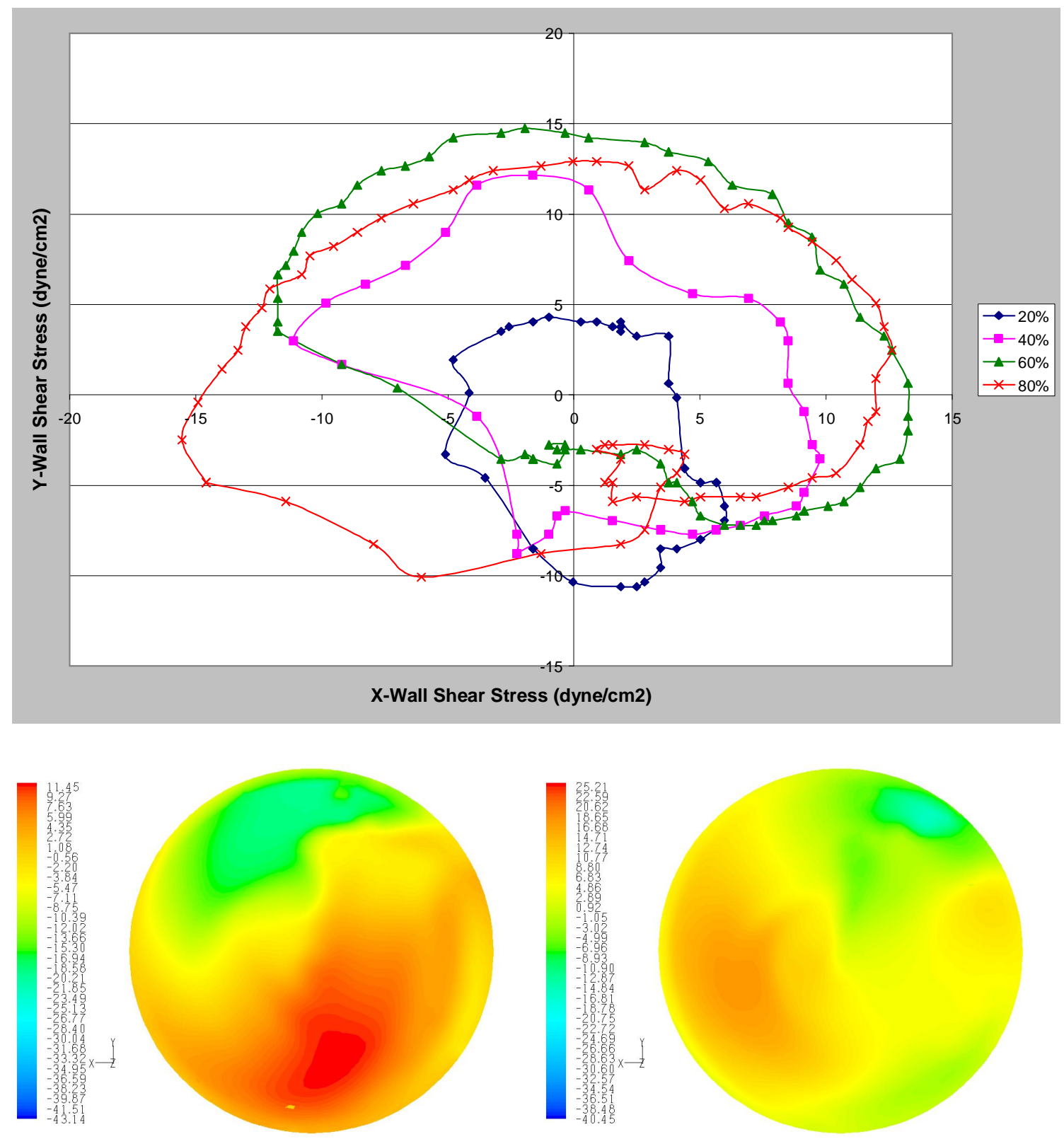

FIGURE 86 - Stokes Number 10 Plot of X and Y WSS Components and X and Y WSS

Contours 

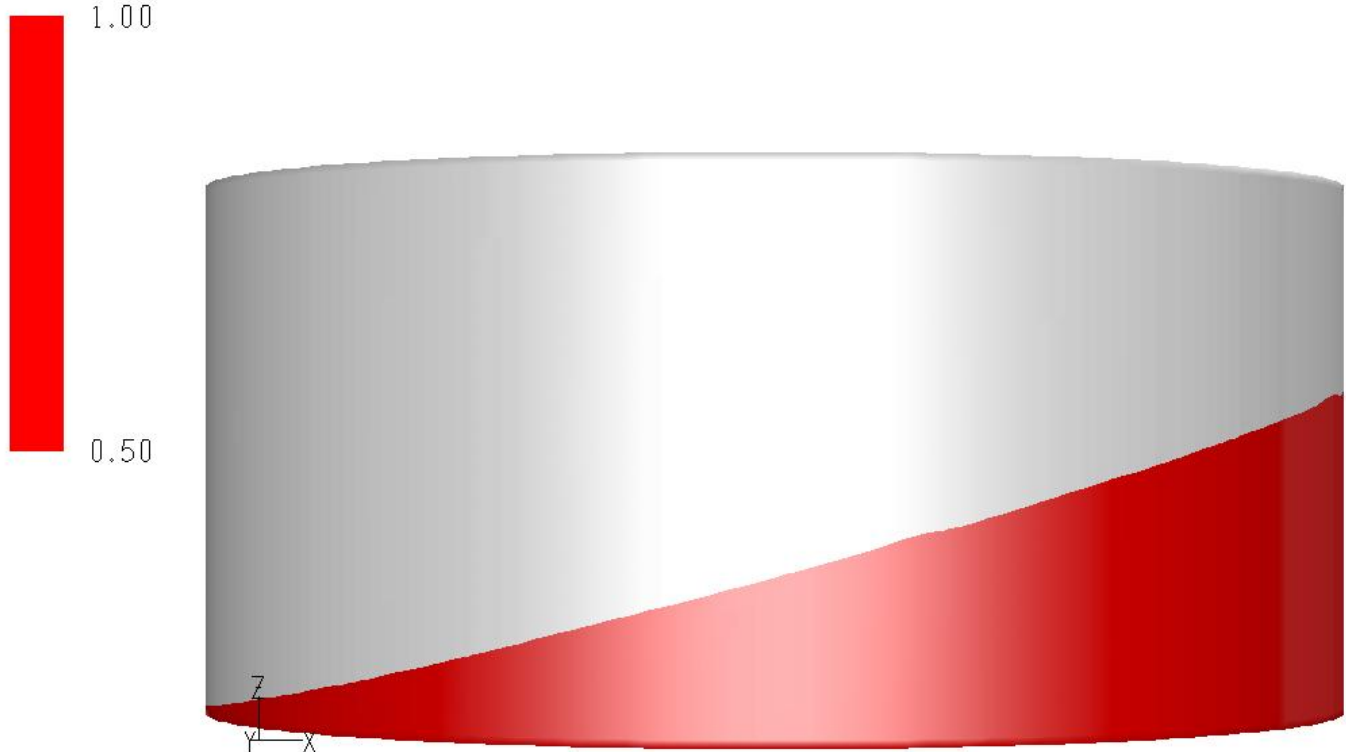

FIGURE 87 - Stokes Number 5.0 with no Slope Effect

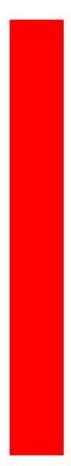

1.00

0.50
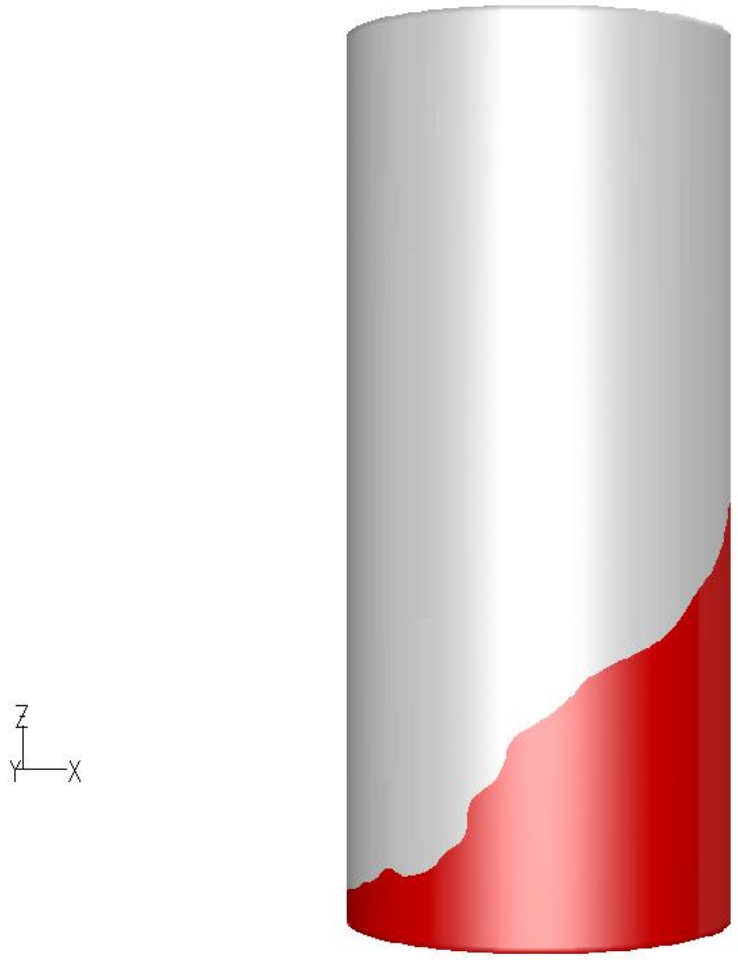

FIGURE 88 - Stokes Number 10 with Slope Effect 


\section{Plot of $X$ and $Y$ Wall Shear Stress Components as a Function of Froude Number}

Plots of X and Y WSS components as Froude Number is varied are analyzed to determine if a visible effect of the Froude Transition is noticeable and how the plots change through the range. Each of the plots of X and Y WSS components of Froude Numbers between 0.1 and 0.2, shown in Figures 89-94, develops a similar pattern signifying no transition occurs in this region. The plot for $20 \%$ does not cross, transitioning from an L shape to an ovoid shape. The plot for $40 \%$ is of oval shape, varying from wide to narrow with Froude Number. Like the plot for $40 \%$, the plot for $60 \%$ is of oval shape with a crossed lobe on the upper right side. The plot for $80 \%$ is a an oval shape, with a hemi-circle cut out of the bottom more towards the right side with a crossing projection out the right side; it changes in thickness as the Froude Number is varied. For the Froude Numbers of 0.3 and 0.4, shown in Figures 95 and 96, the contours change. The plot for $20 \%$ shows an oval shape, $40 \%$ shows an oval with some crossing, $60 \%$ makes a figure eight shape, and $80 \%$ is an oval with a long protrusion from the right side, being flat on the bottom. A differing plot of X and Y WSS components is present for 0.3 from that of 0.2 showing that a transition occurs between 0.3 and 0.2 ; the differences are seen in an inspection of Figures 17 and 18 showing the Froude Transition. At the Froude Number of 0.5, shown in Figure 97, there are a few minor differences from that of 0.4 . The plot of $20 \%$ crosses in figure eight, $40 \%$ also makes a figure eight, $60 \%$ makes an oval shape with a protrusion to the upper right, and $80 \%$ makes an oval shape with a protrusion to the upper right and a crossing lobe on the bottom left. Although the shape of the plots is different, visual inspection shows that no Stokes or Slope effects occur. Actually, a deeper inspection shows that at the Froude number of 0.1 , shown in 
Figure 98, Stokes effects are present. This shows that Stokes effects are present throughout the entire range of Froude Numbers studied. Similarly, inspection of the Froude Number of 1.0, shown in Figure 99, indicates that a Slope effect is not present. This illustrates that no Slope Effects are present in the range of Froude Numbers studied. The change in plots is then caused by the different appearance of the standing wave at the leading edge of the wave as seen in Figures 24 and 25. The Froude Number of 0.7 and 1.0, shown in Figures 100 and 101, are similar although there are again slight differences from that of 0.5 . The plot shows that at $20 \%$ an $\mathrm{S}$ shape develops, crossing at both ends, at $40 \%$ there is a triangular shape that crosses at its ends, at $60 \%$ and $80 \%$ there are oval shapes with protrusions on the right side. Again the difference in 0.5 and 0.7 is due to the difference in the shape of the standing wave at the leading edge of the wave as the Stokes and Slope transitions are not in effect. 

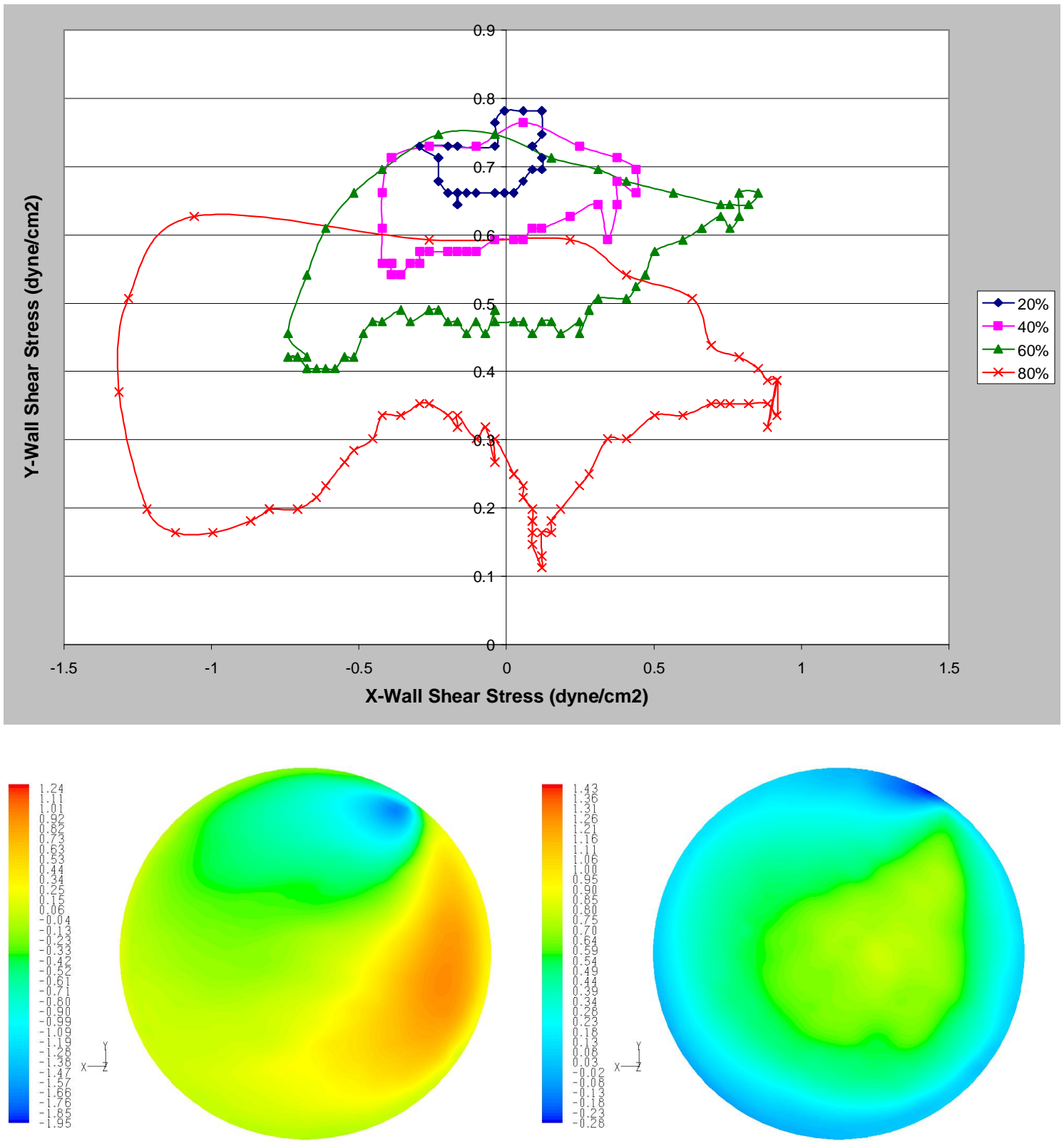

FIGURE 89 - Froude Number 0.1 Plot of X and Y WSS Components and X and Y WSS

Contours 

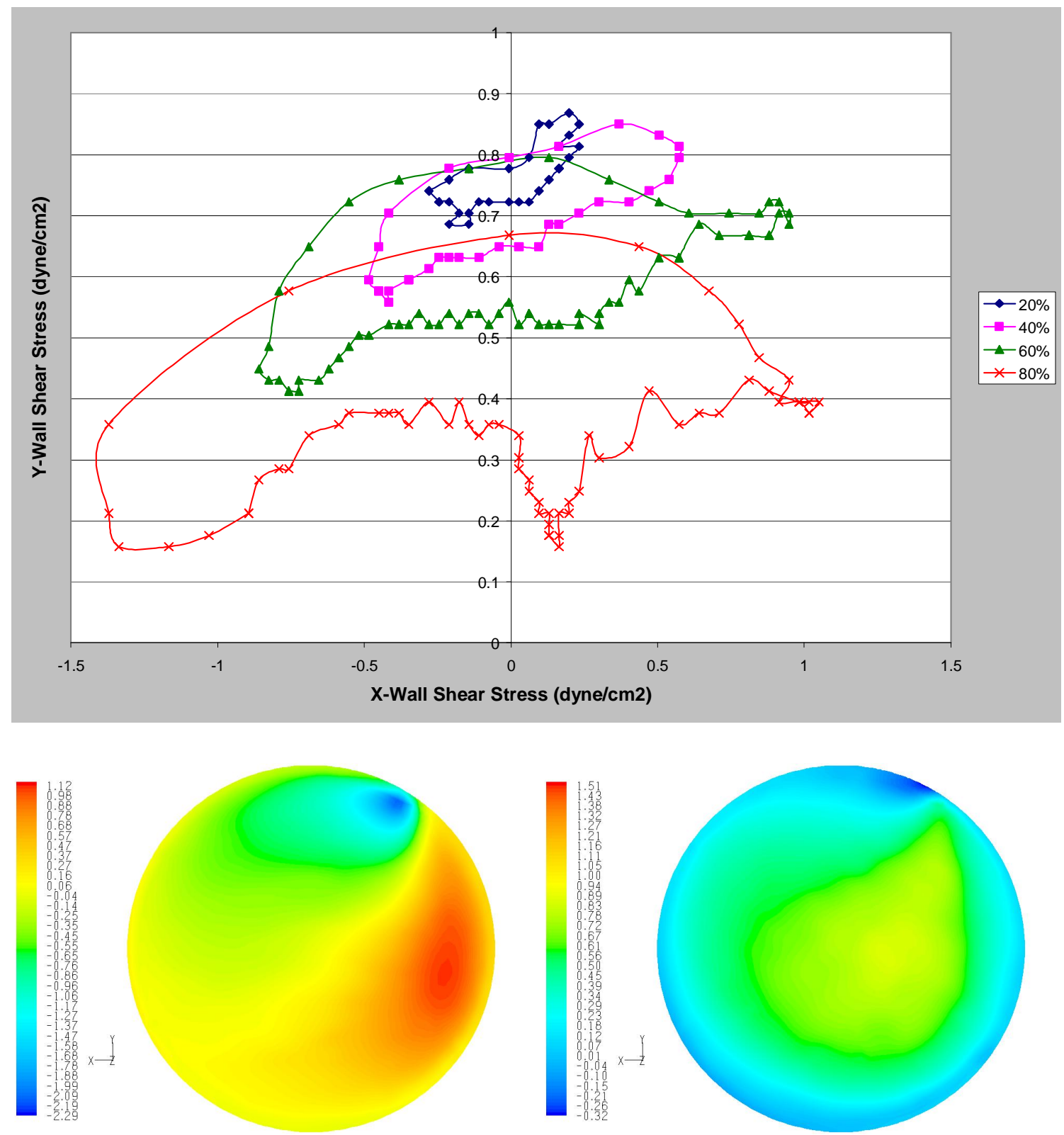

FIGURE 90 - Froude Number 0.12 Plot of X and Y WSS Components and X and Y WSS Contours 

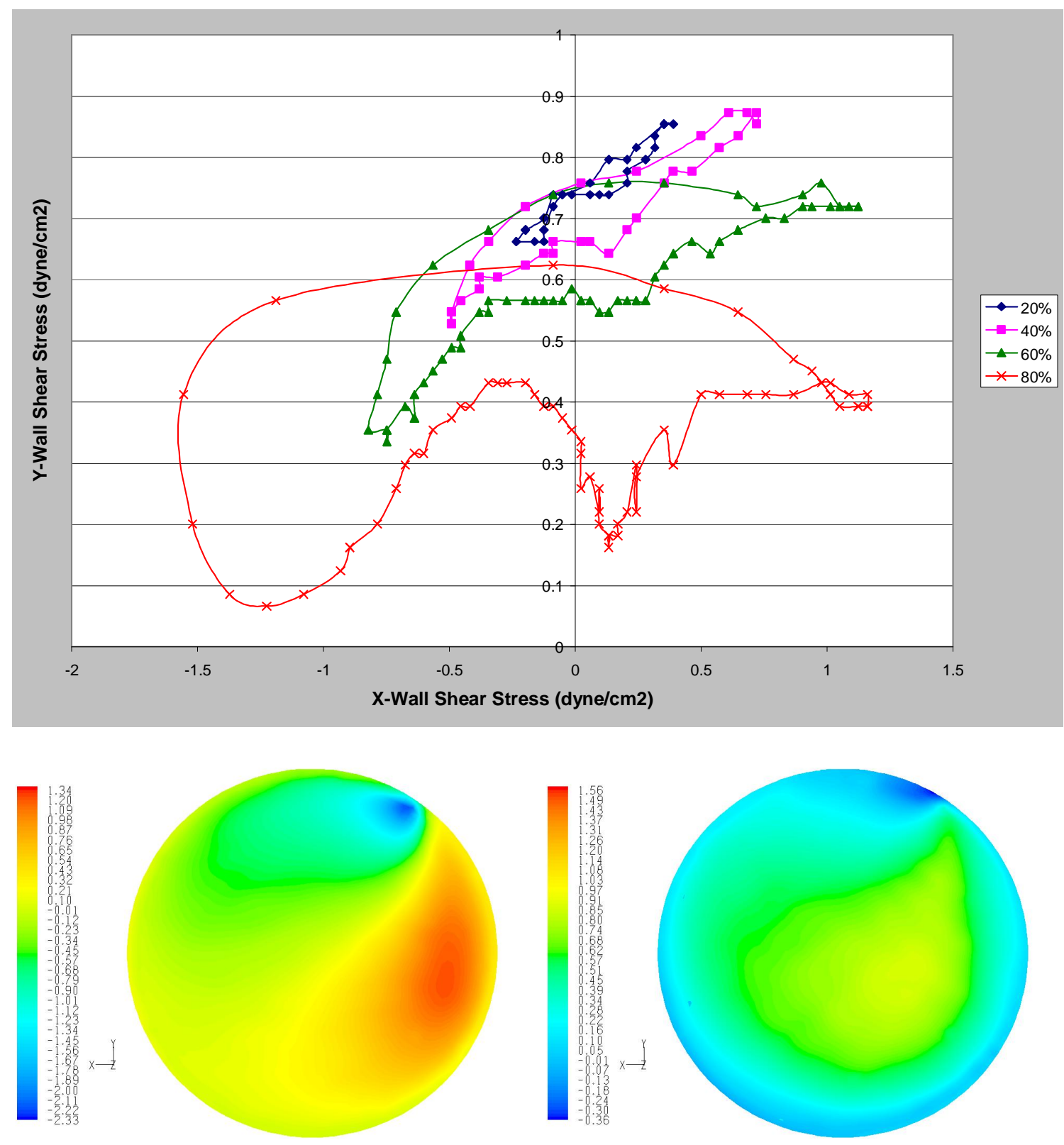

FIGURE 91 - Froude Number 0.14 Plot of X and Y WSS Components and X and Y WSS Contours 

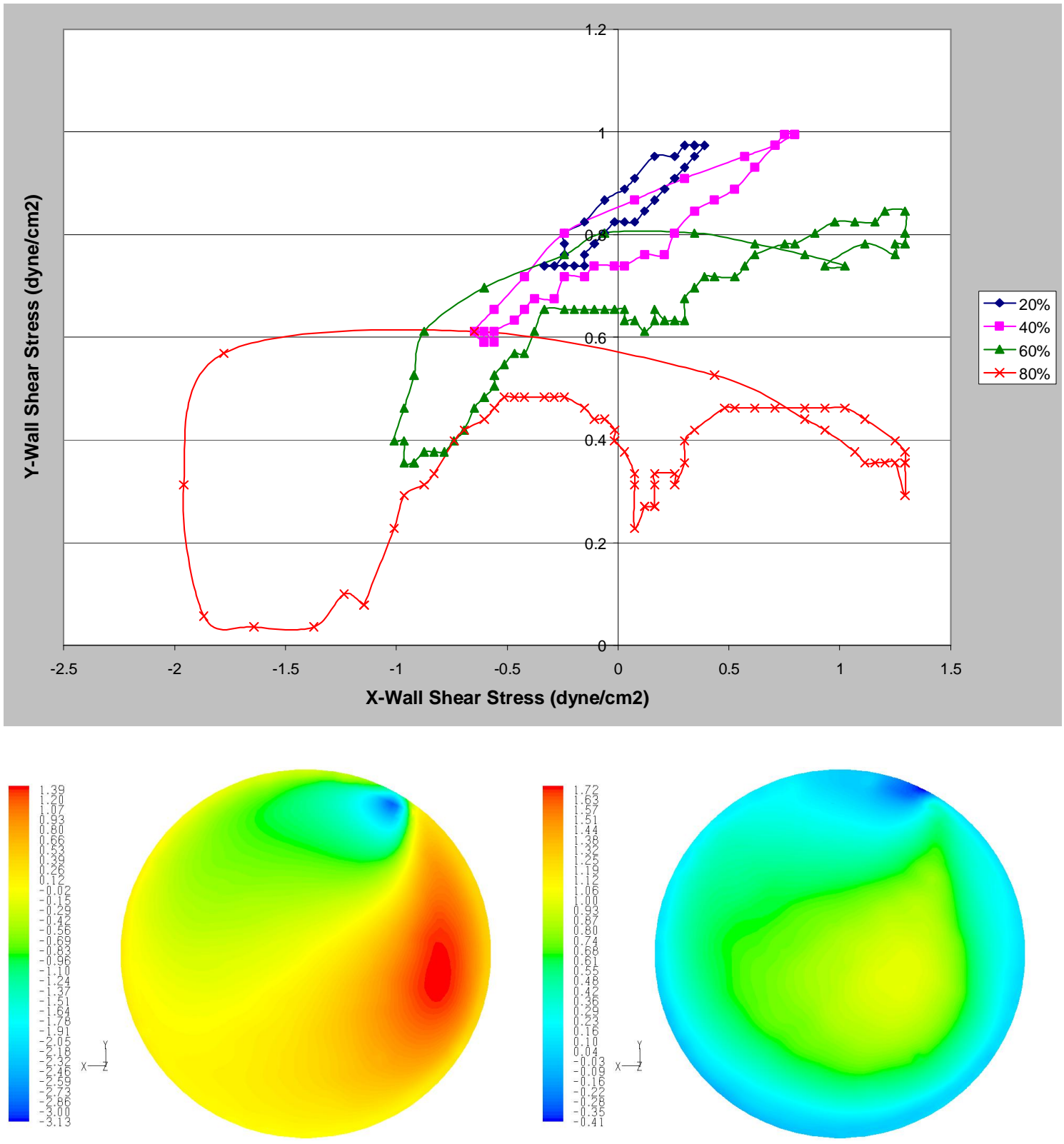

FIGURE 92 - Froude Number 0.16 Plot of X and Y WSS Components and X and Y WSS Contours 

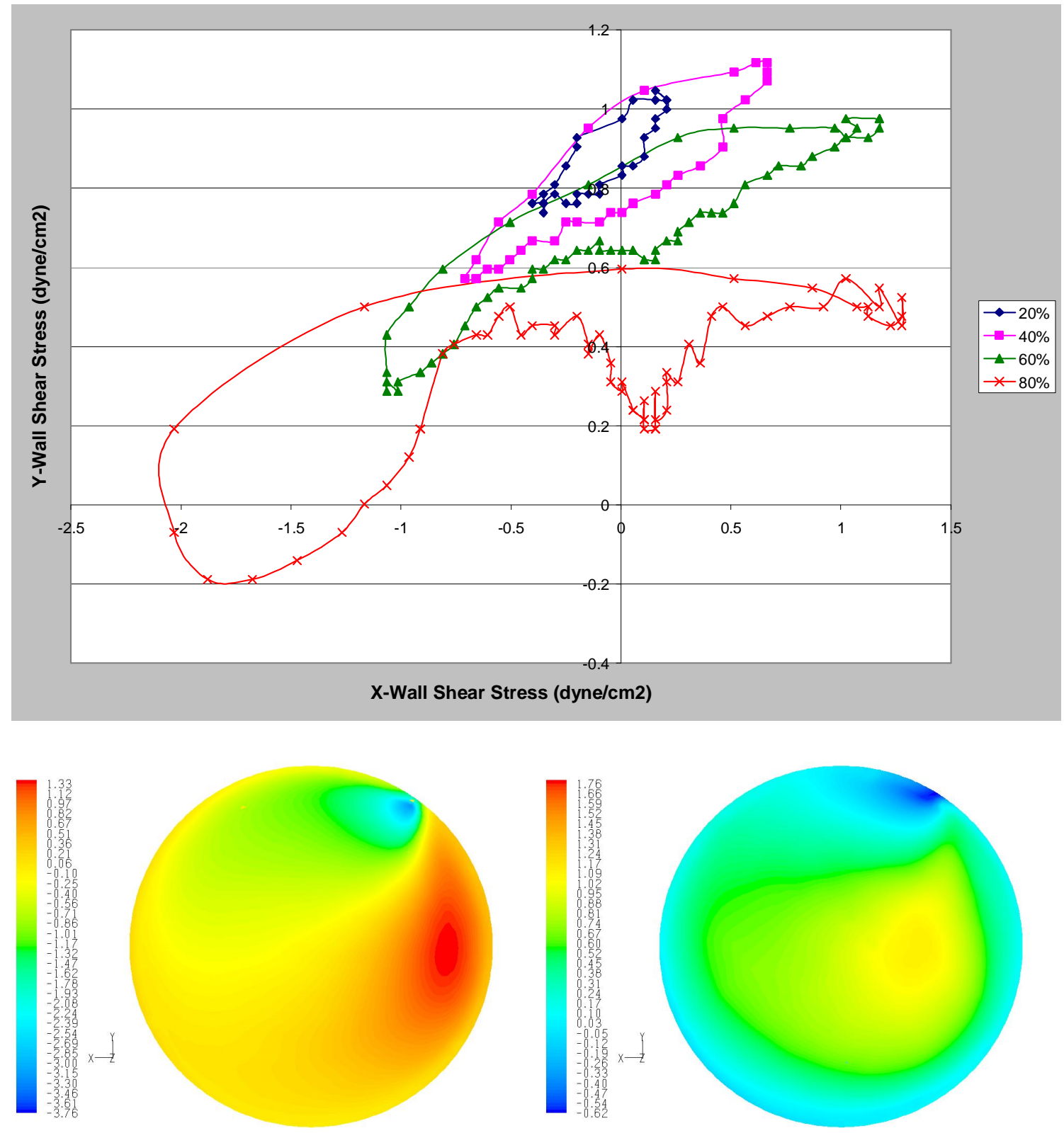

FIGURE 93 - Froude Number 0.18 Plot of X and Y WSS Components and X and Y WSS Contours 

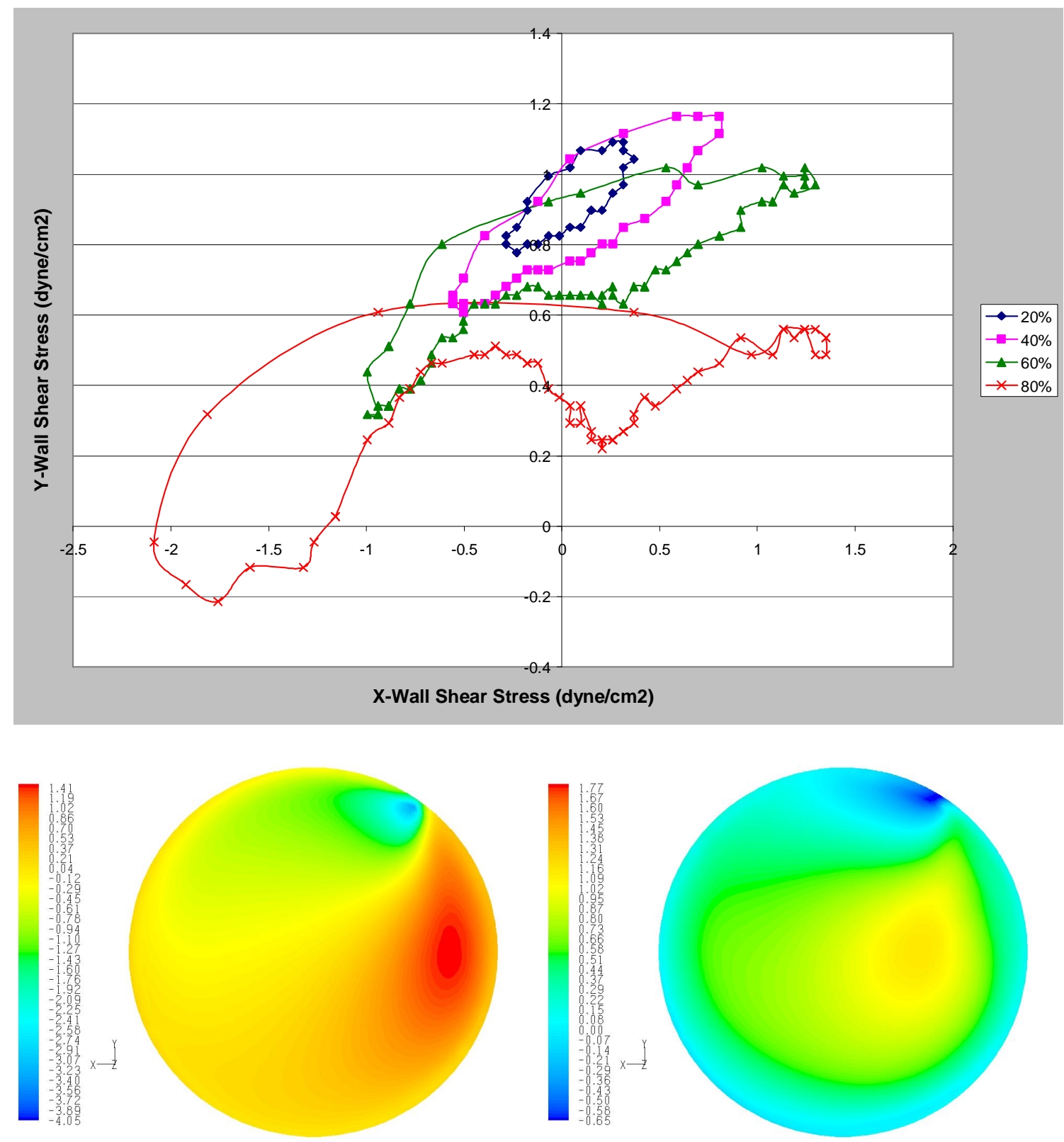

FIGURE 94 - Froude Number 0.2 Plot of X and Y WSS Components and X and Y WSS

Contours 

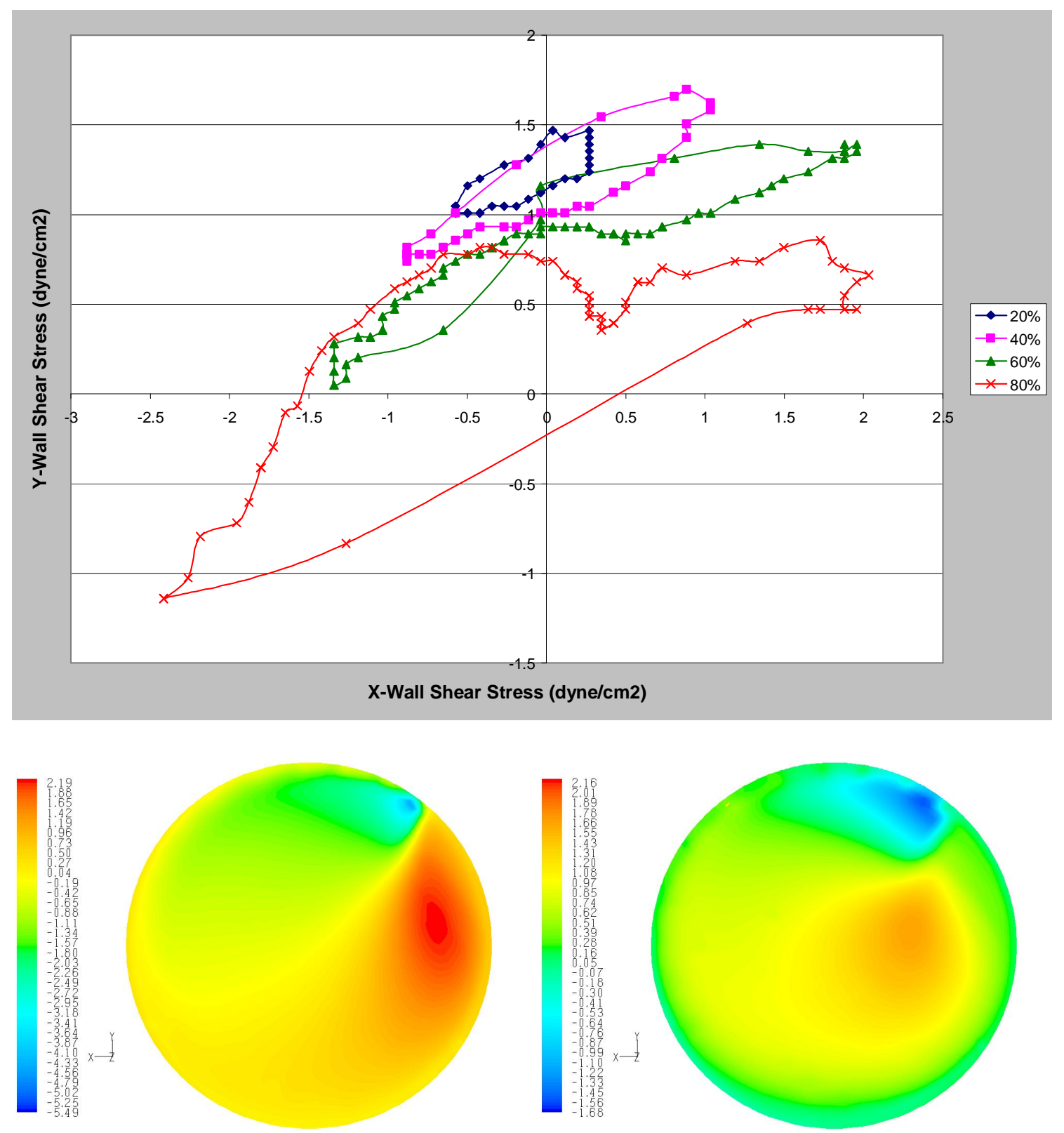

FIGURE 95 - Froude Number 0.3 Plot of X and Y WSS Components and X and Y WSS

Contours 

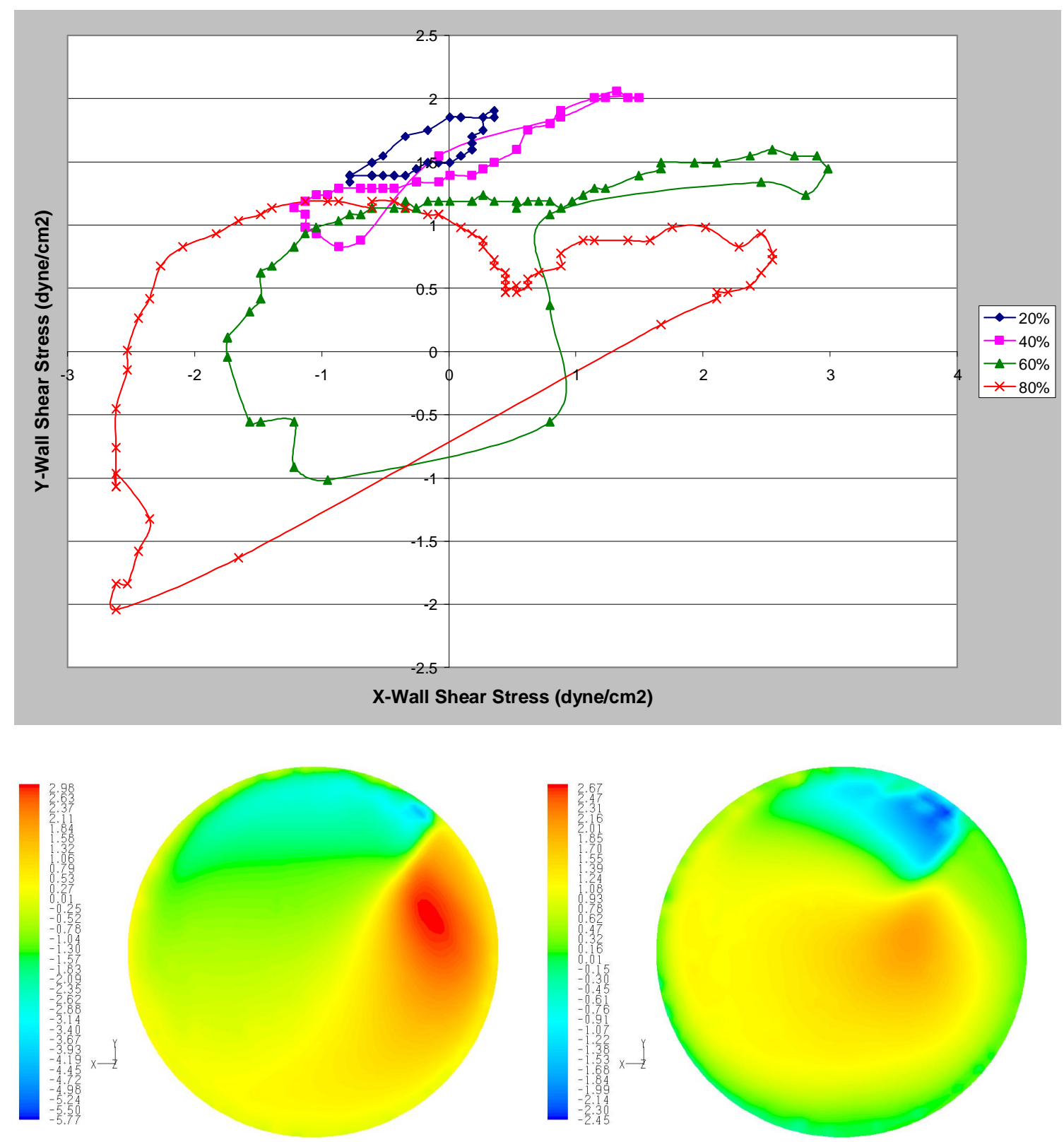

FIGURE 96 - Froude Number 0.4 Plot of X and Y WSS Components and X and Y WSS

Contours 

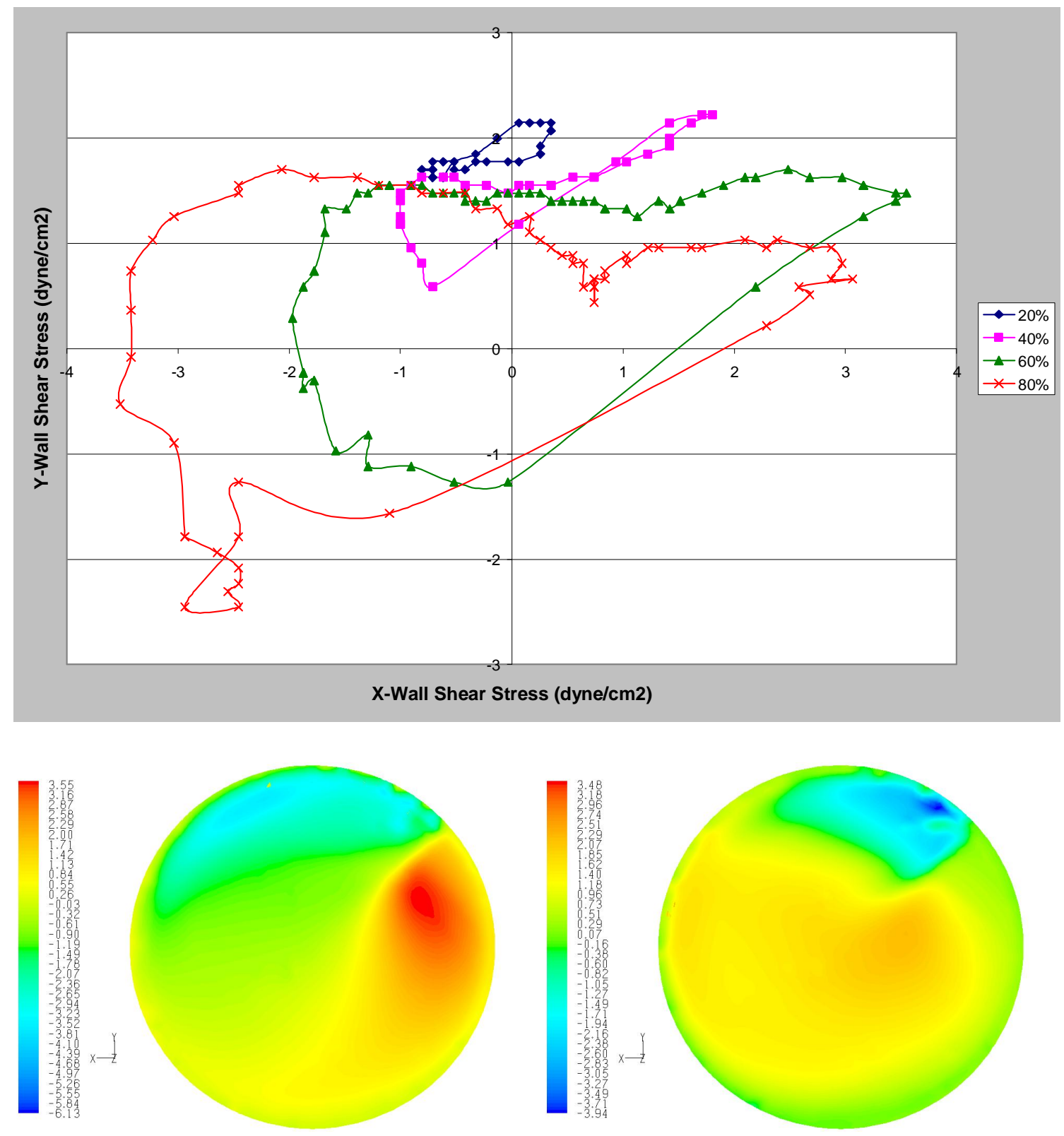

FIGURE 97 - Froude Number 0.5 Plot of X and Y WSS Components and X and Y WSS

Contours 


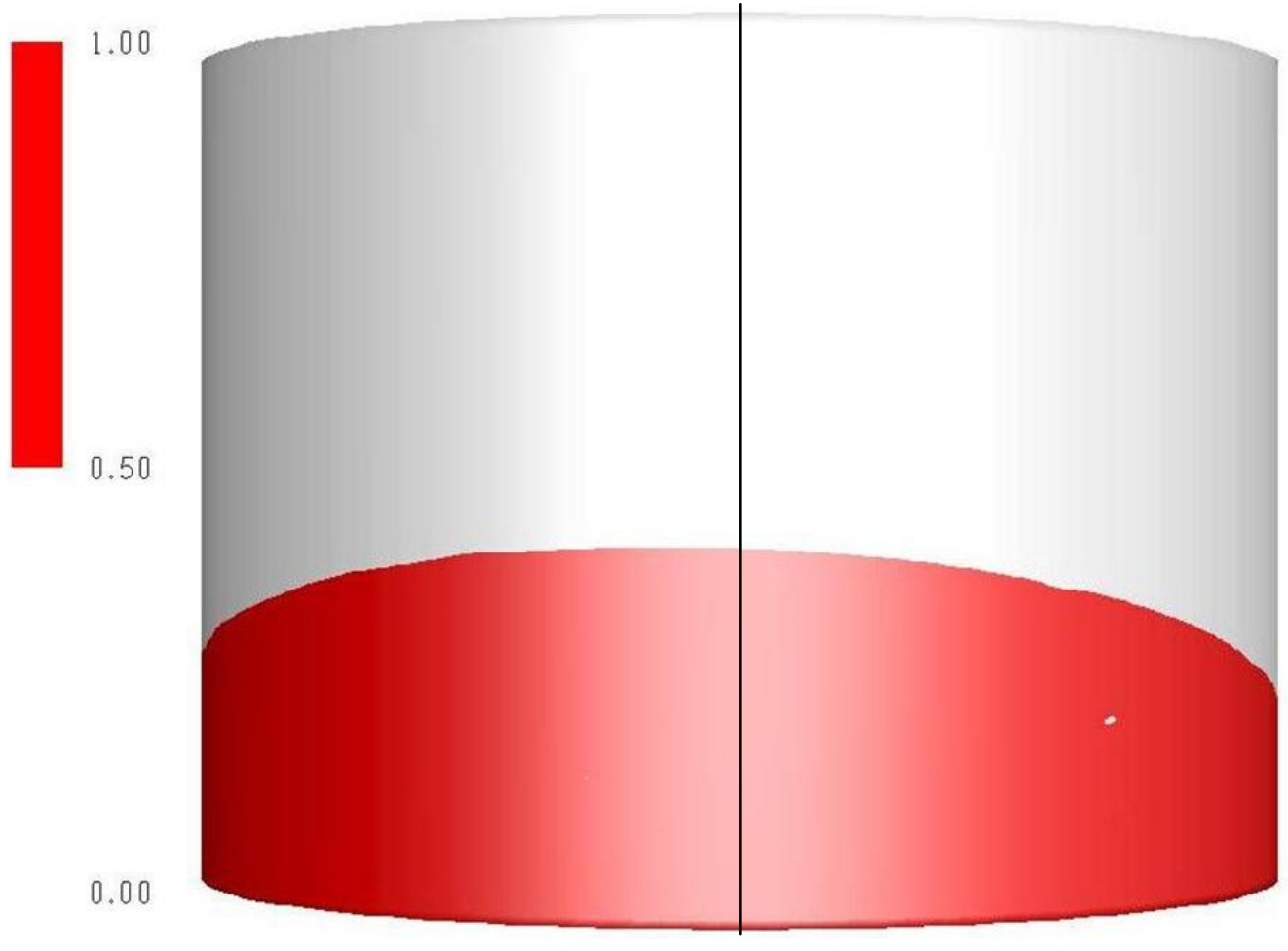

FIGURE 98 - Froude Number 0.1 with Stokes Effect Present
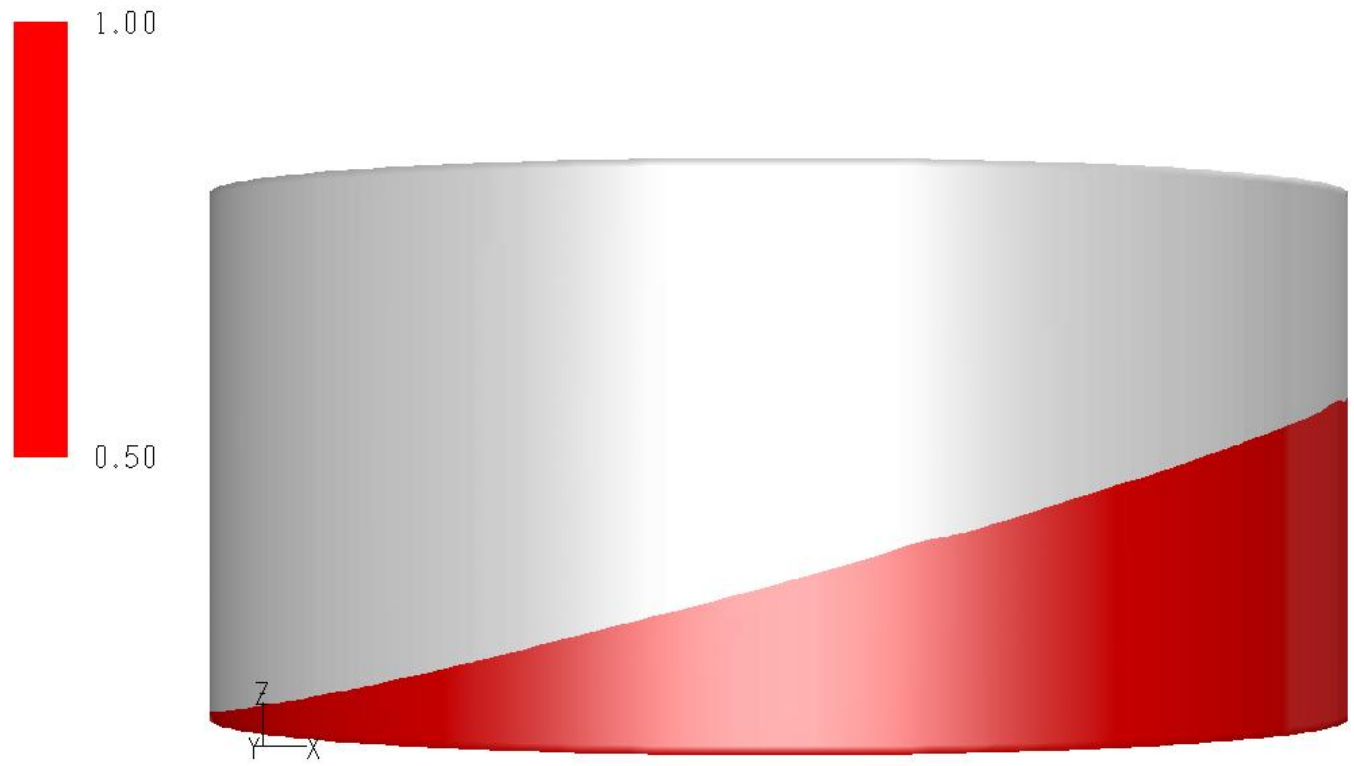

0.00

FIGURE 99 - Froude Number 1.0 with no Slope Ratio Effect Present 

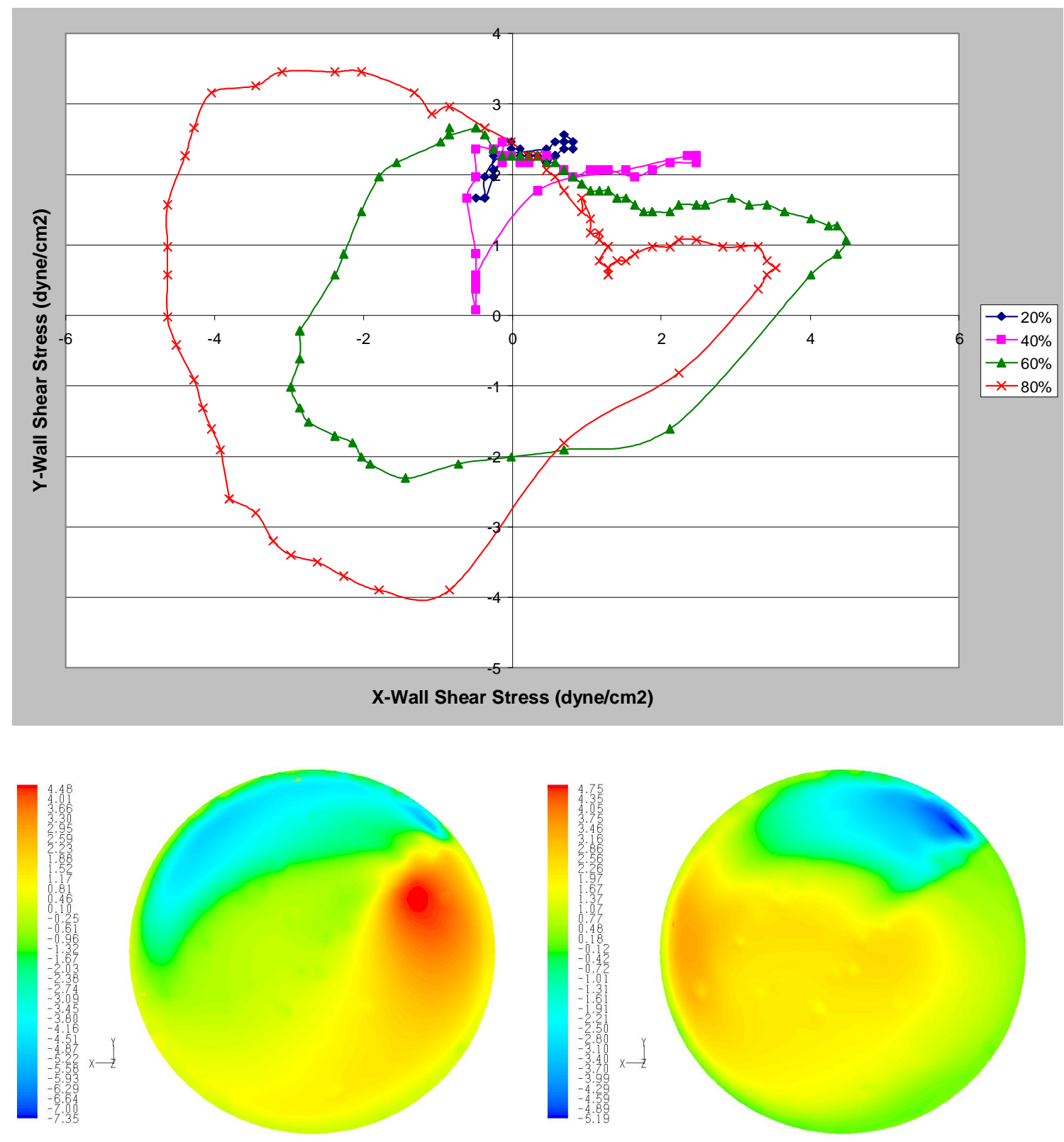

FIGURE 100 - Froude Number 0.7 Plot of X and Y WSS Components and X and Y WSS Contours 

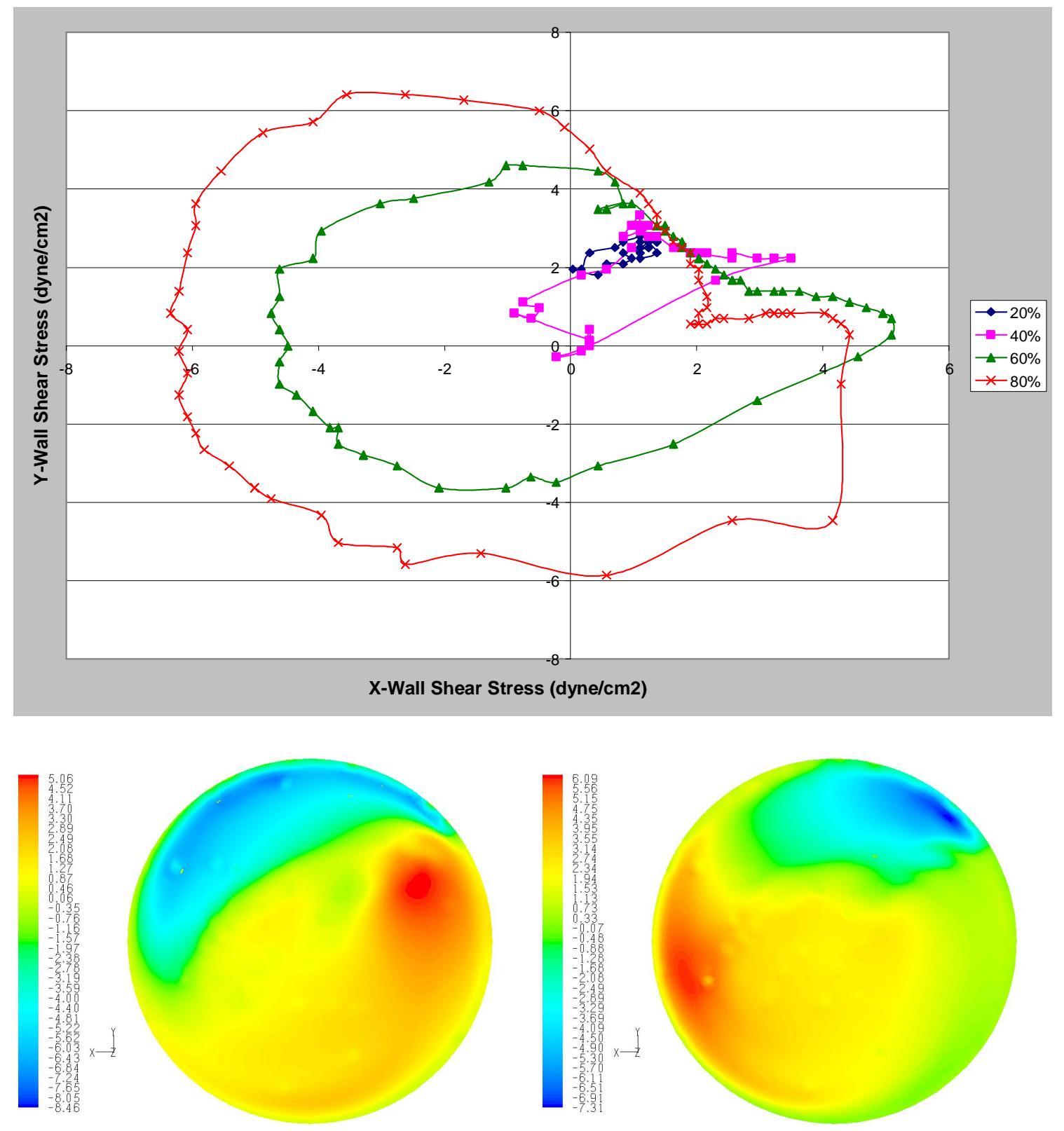

FIGURE 101 - Froude Number 1.0 Plot of X and Y WSS Components and X and Y

WSS Contours

\section{Plots of $X$ and $Y$ Wall Shear Stress Components as a Function of Slope Ratio}

Plots of X and Y WSS components as Slope Ratio is varied are analyzed to determine if a visible effect of the Slope transition is noticeable and how the plots change through the range. Although plots of X and Y WSS components are present for the Slope 
Ratios of 0.1 and 0.2, shown in Figures 102 and 103, definitive results are difficult to define from these cases since it is impossible to accurately make a visual inspection for transitions with four peaks present. In addition, due to the unique nature of the WSS contours, the plots cross many times making it difficult to accurately describe. For the Slope Ratio of 0.5 , seen in Figure 104, the $20 \%$ and $40 \%$ plots make ovoid shapes. The plot for $60 \%$ makes a long thin shape resembling a carrot. The plot at $80 \%$ makes an ovoid shape, with heart shaped crossings at both ends. For a Slope Ratio of 0.8, seen in Figure 105, the plot of X and Y WSS components are much different than those from 0.5. The plot for $20 \%$ is in the shape of a figure eight, $40 \%$ is in the shape of a triangle with crossings at each protrusion, $60 \%$ is in ovoid shape with a protrusion on the right side and crossings on the left top and bottom, and $80 \%$ is oval in shape with a crossing on the right side. After examining the free surface plots for 0.5 and 0.8, Figures 106 and 107, the difference in the plots is found to be related to an effect similar to a Stokes transition. For a Slope Ratio of 1.1, seen in Figure 108, the plot of X and Y WSS components again is not similar to those at 0.8 . The plot for $20 \%$ shows a figure eight shape with multiple crossings, $40 \%$ shows a rectangular shape, $60 \%$ shows an ovoid shape, and $80 \%$ shows an ovoid shape with a protrusion on the right. Since the elliptical plots for 0.8 and 1.1 are not similar, this indicates that a transition occurs. It has been previously determined that an effect resembling the Froude transition occurs between these values. Slope Ratios of 1.4, 1.7 an 2.0, shown in Figures 109-111, all have similar plots of X and Y WSS components, although they differ greatly from the plot of the Slope Ratio of 1.1. The plot for $20 \%$ shows an ovoid shape with a protrusion on the bottom right, $40 \%$ shows an ovoid shape, $60 \%$ shows an ovoid shape, and $80 \%$ shows on ovoid shape with a 
protrusion on the bottom right and a crossing on the bottom left. The difference in 1.1 and 1.4 was previously attributed to the Slope transition.
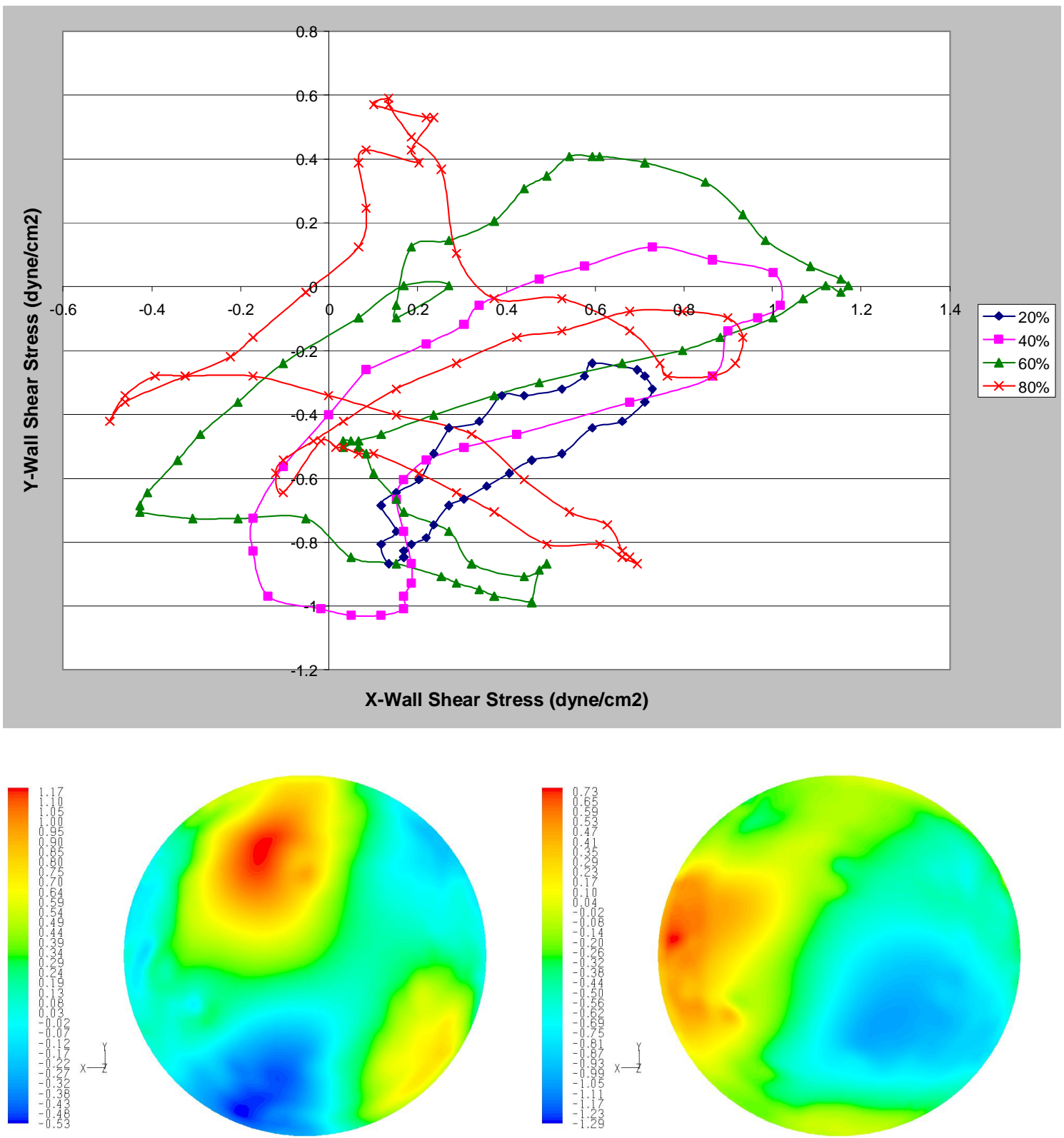

FIGURE 102 - Slope Ratio 0.1 Plot of X and Y WSS Components and X and Y WSS

Contours 

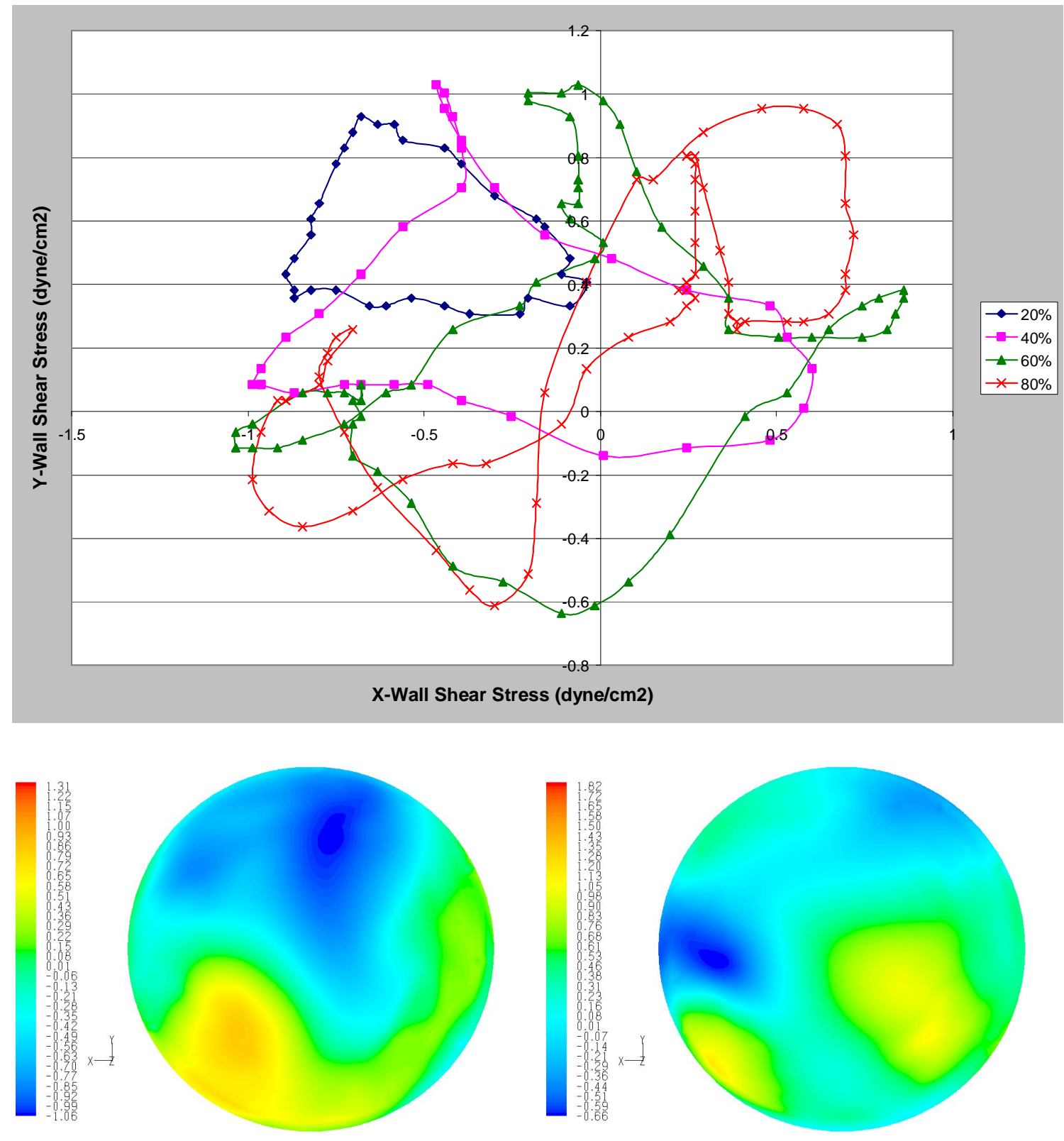

FIGURE 103 - Slope Ratio 0.2 Plot of X and Y WSS Components and X and Y WSS

Contours 

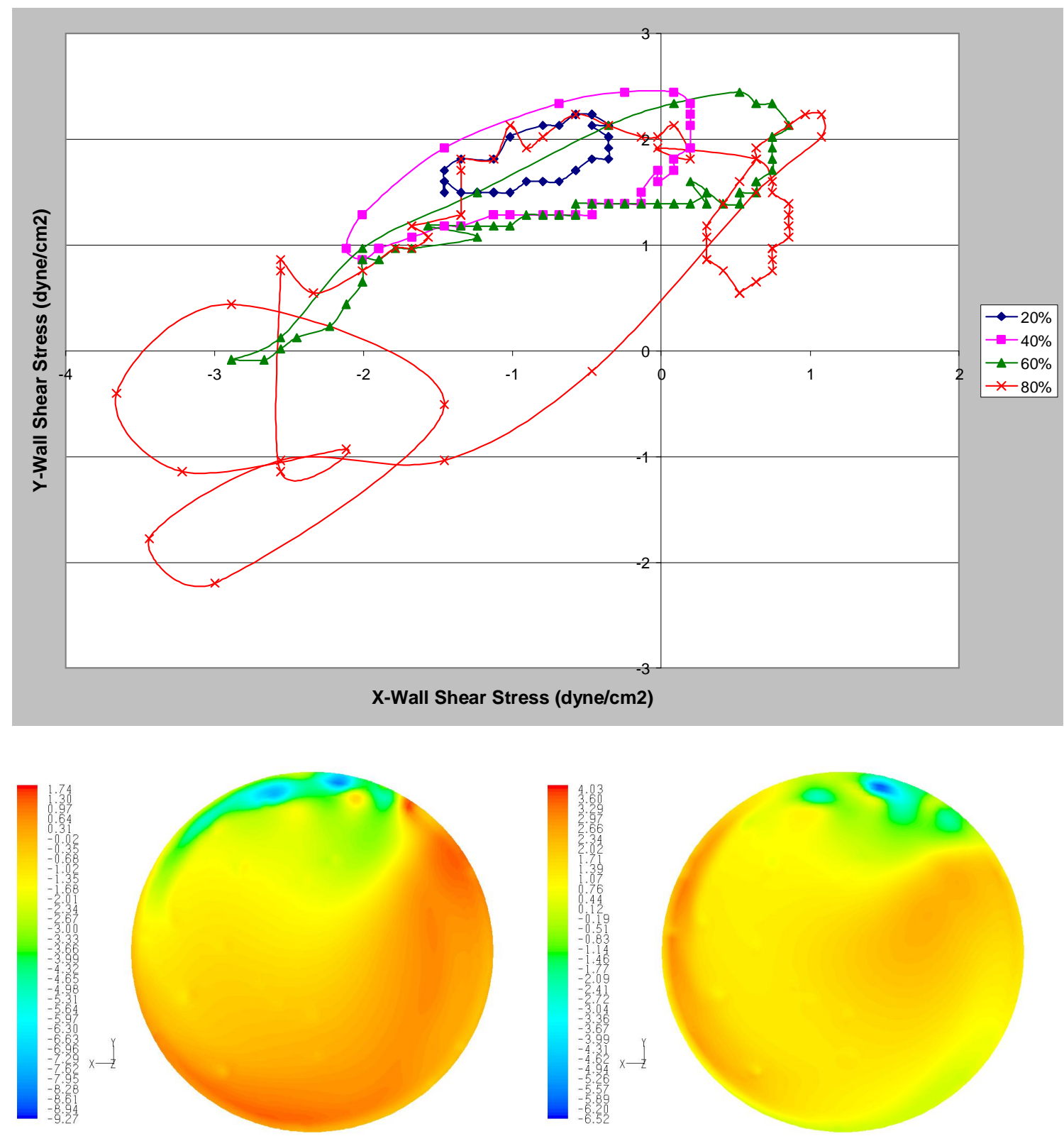

FIGURE 104 - Slope Ratio 0.5 Plot of X and Y WSS Components and X and Y WSS

Contours 

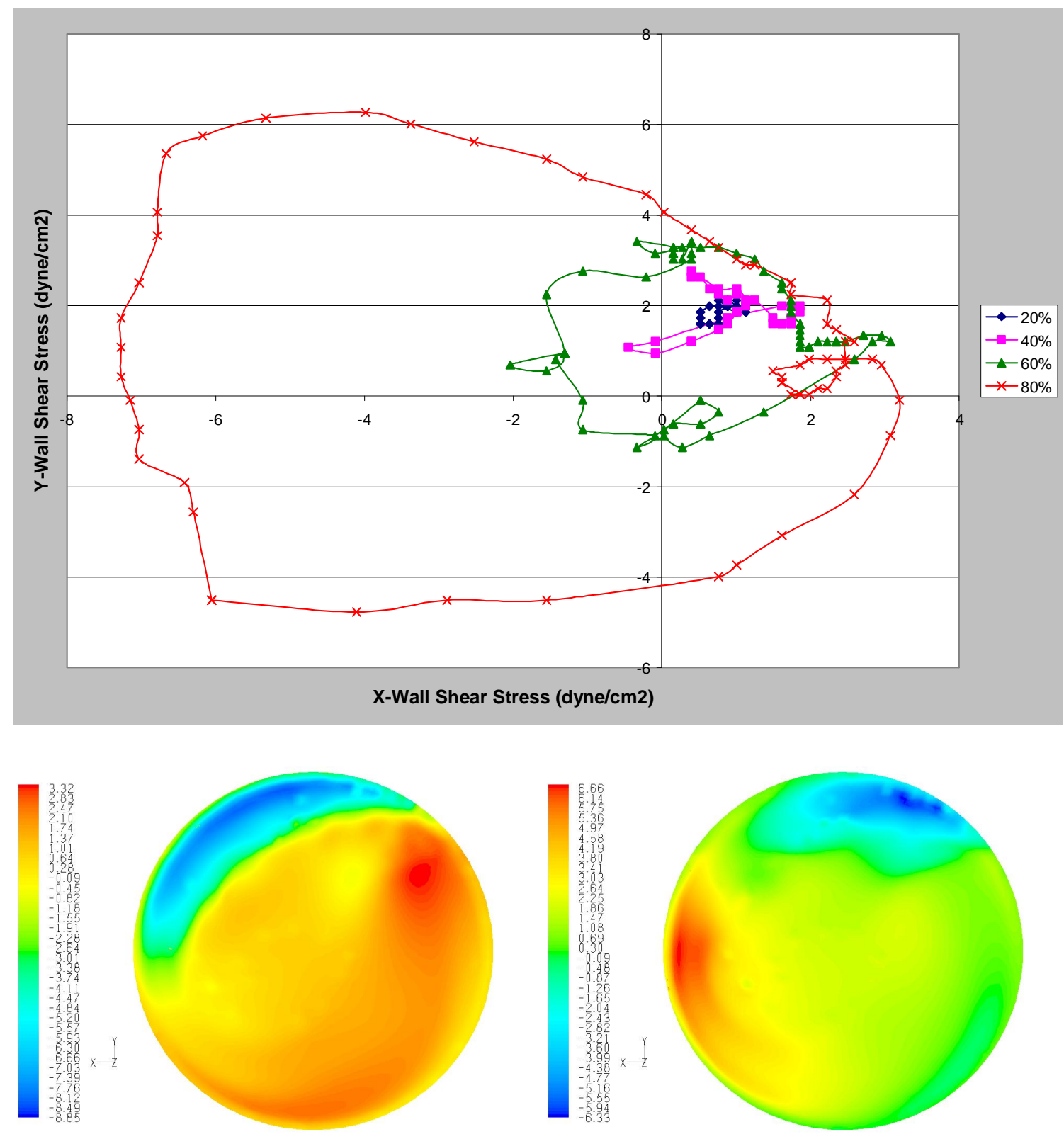

FIGURE 105 - Slope Ratio 0.8 Plot of X and Y WSS Components and X and Y WSS

Contours 


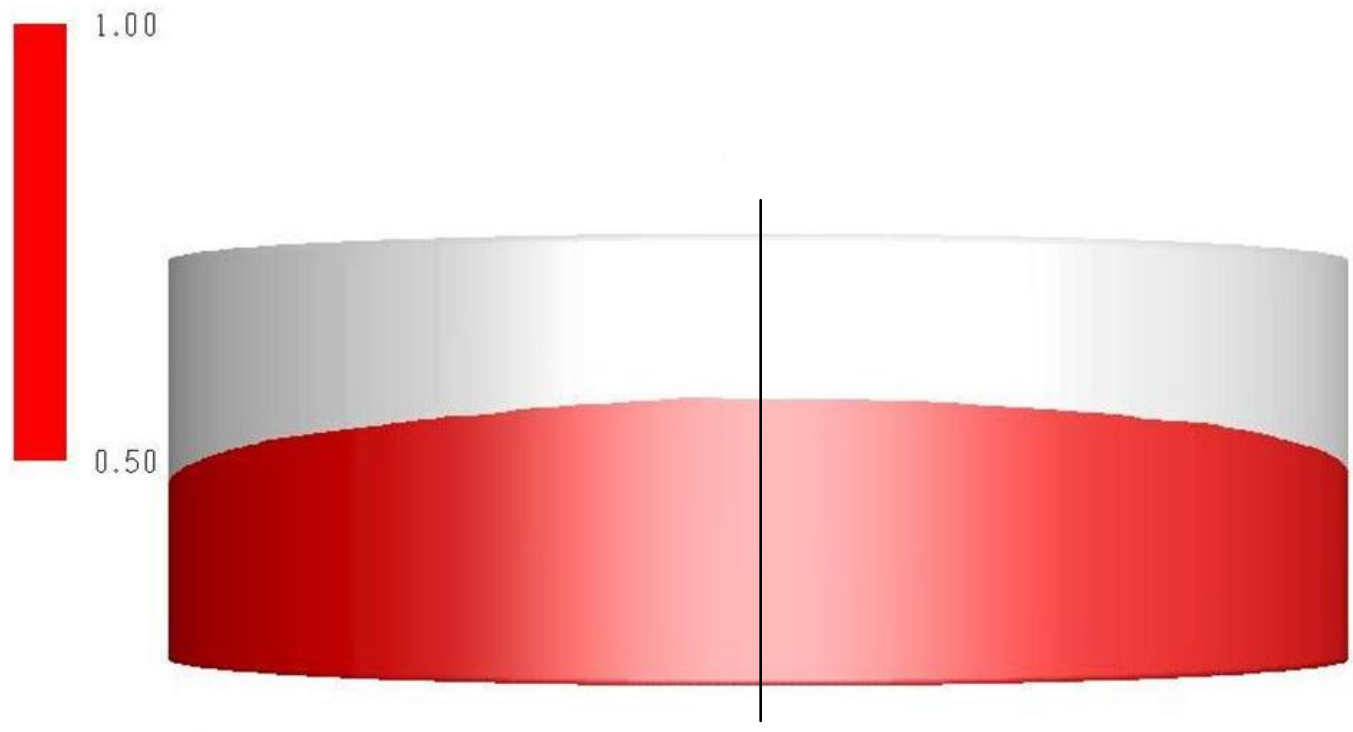

0.00

FIGURE 106 - Slope Ratio 0.5 with No Visible Stokes Effect

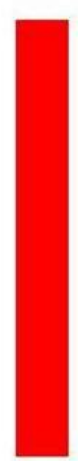

\subsection{0}

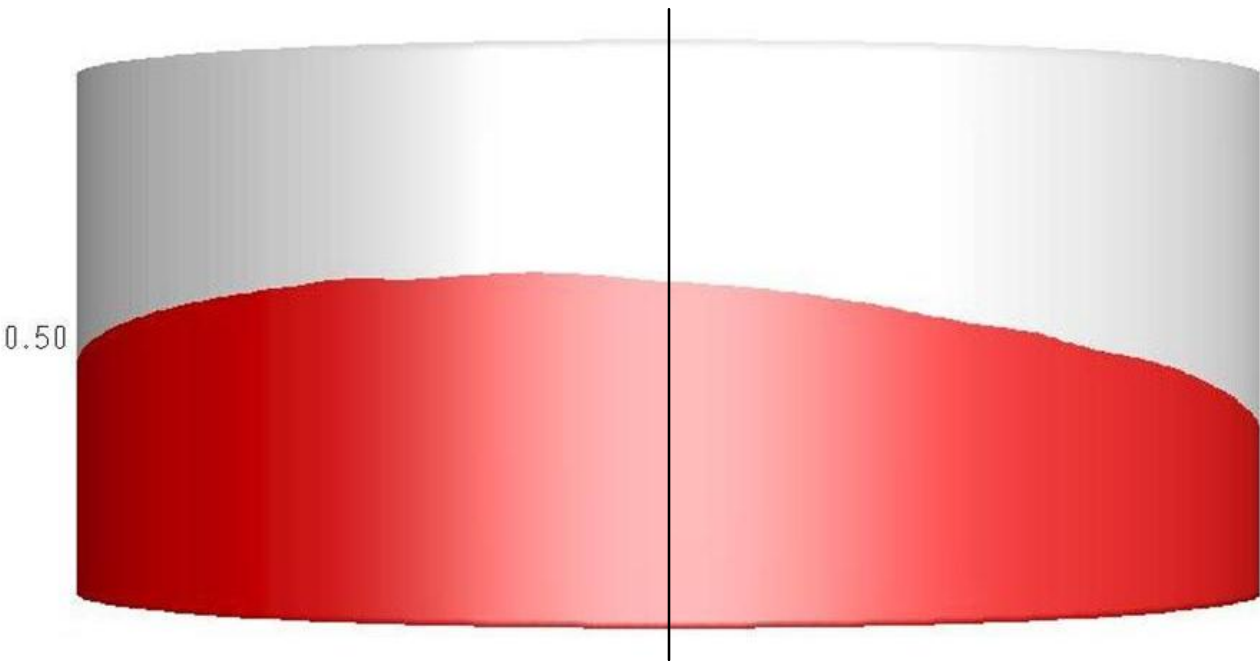

0.00

FIGURE 107 - Slope Ratio 0.8 with Visible Stokes Effect 

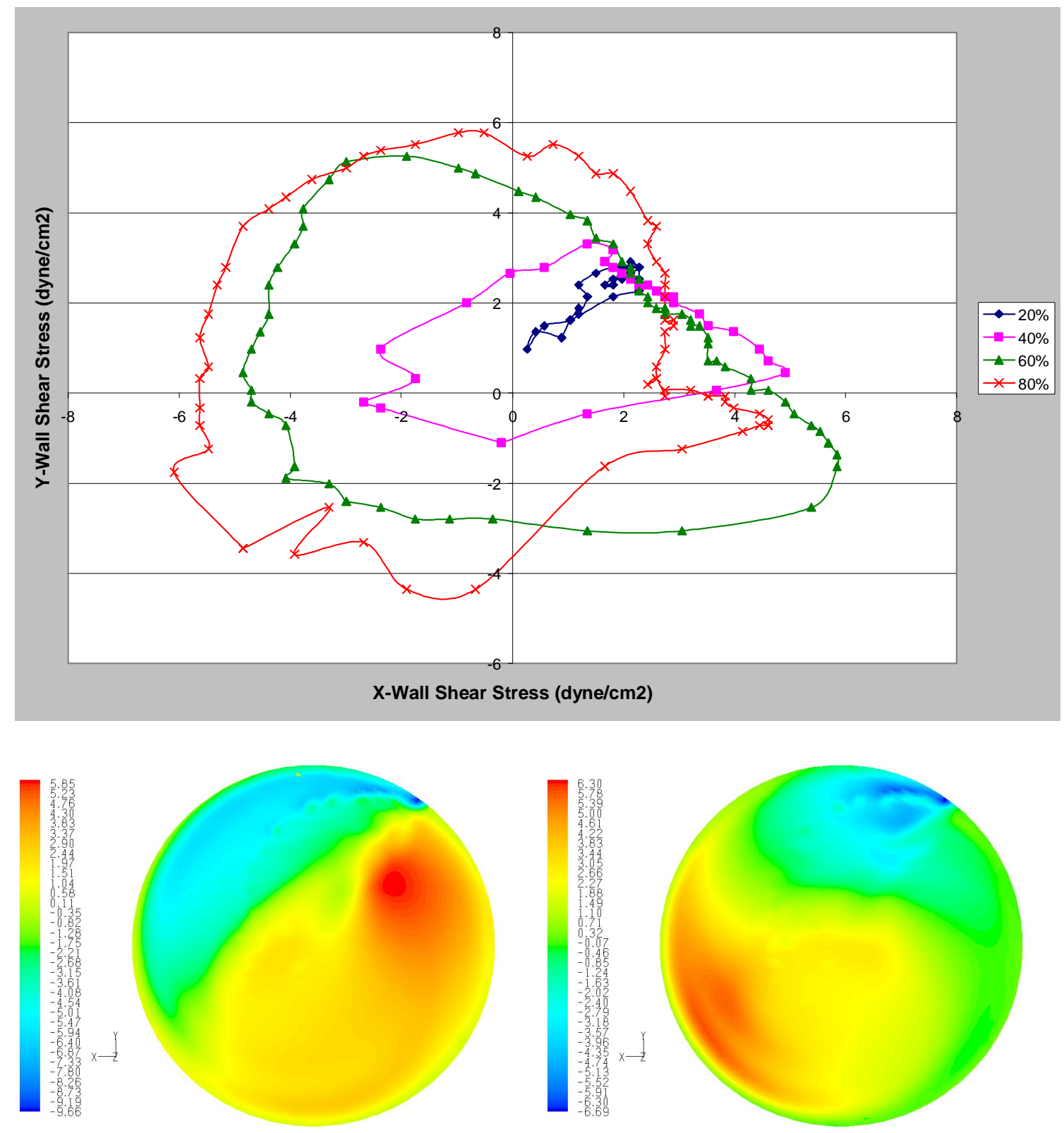

FIGURE 108 - Slope Ratio 1.1 Plot of X and Y WSS Components and X and Y WSS

Contours 

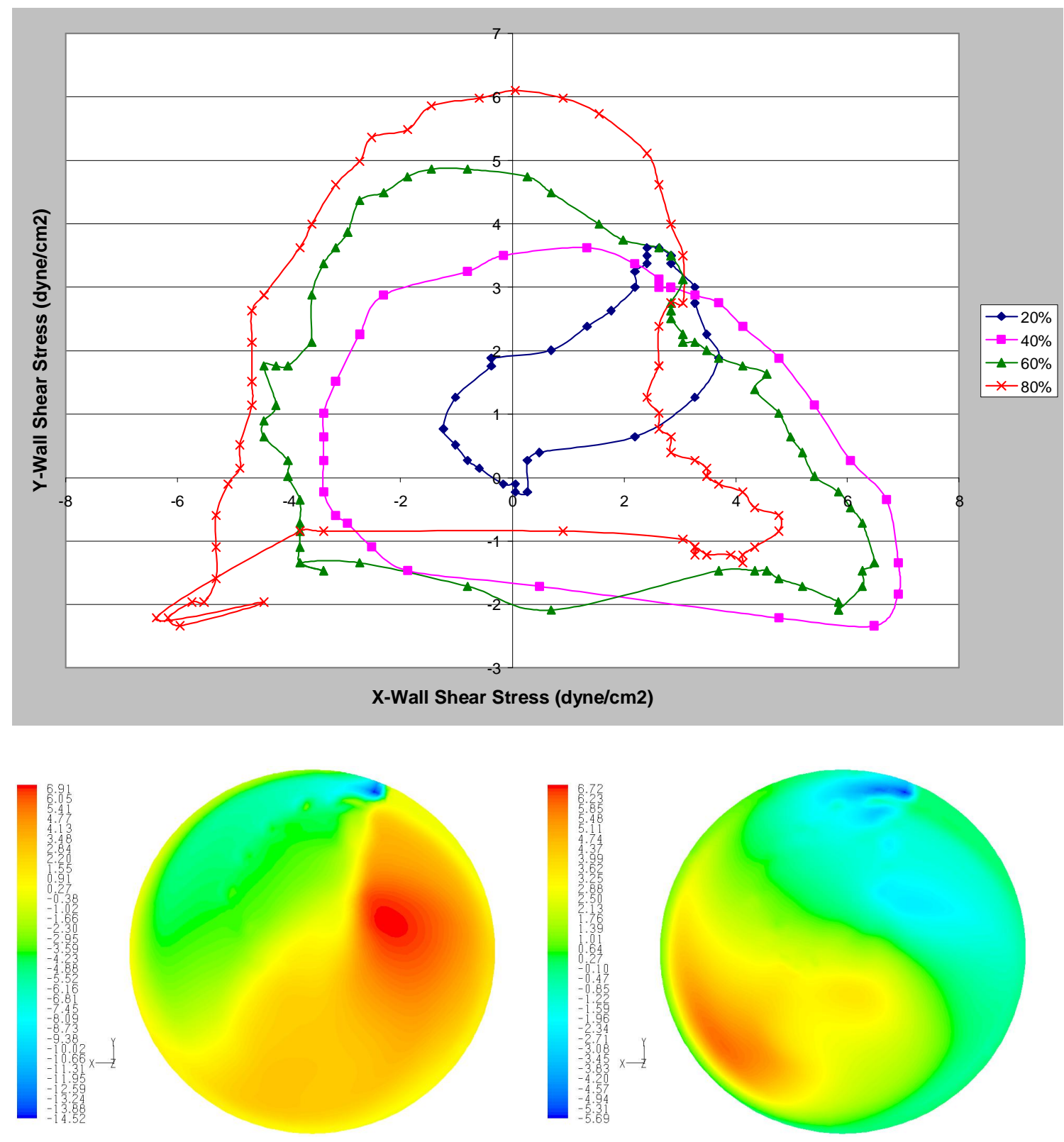

FIGURE 109 - Slope Ratio 1.4 Plot of X and Y WSS Components and X and Y WSS

Contours 

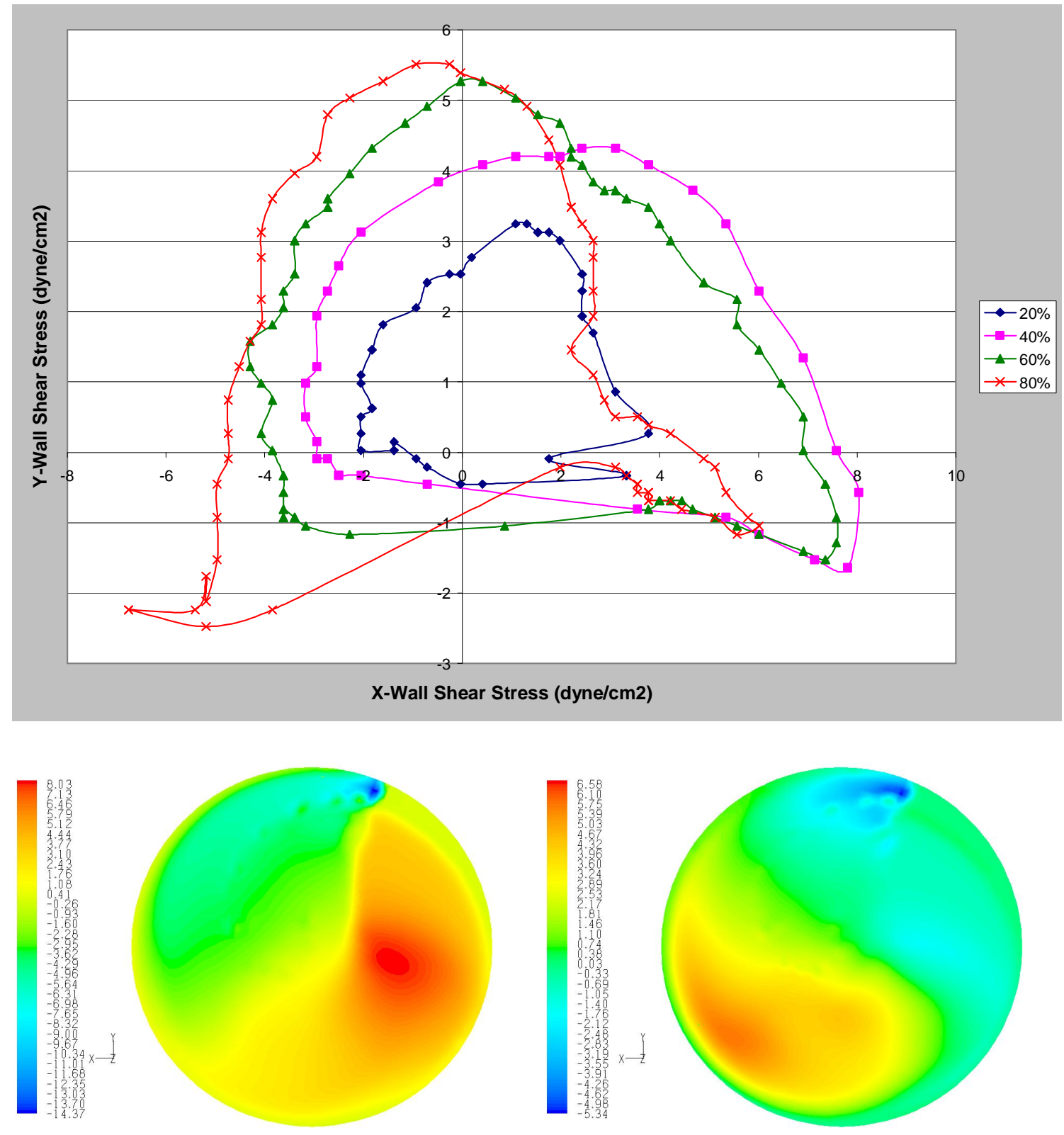

FIGURE 110 - Slope Ratio 1.7 Plot of X and Y WSS Components and X and Y WSS

Contours 

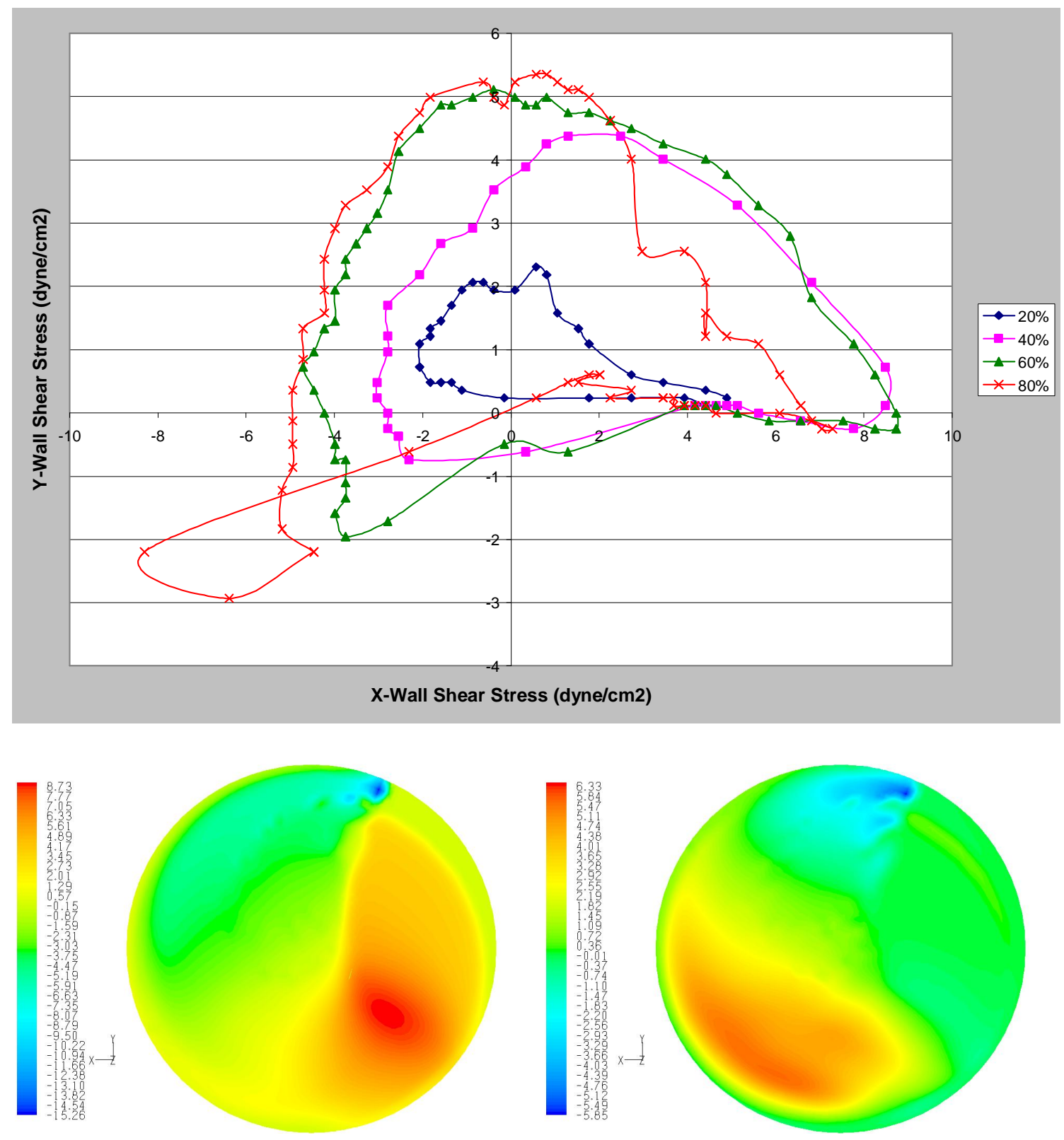

FIGURE 111 - Slope Ratio 2.0 Plot of X and Y WSS Components and X and Y WSS

Contours 


\section{CONCLUSIONS}

Conclusions develop from the findings of the Stokes Number, the Froude number, and the Slope Ratio. The transitions for the dimensionless parameters occur at the following locations:

- The Stokes transition occurs at a value of $4.1 \pm 0.1$ for a Froude number of 1.0 and a Slope Ratio of 1.0.

- The Froude transition occurs at $0.25 \pm 0.05$ for a Stokes Number of 5.0 and a Slope Ratio of 1.0.

- The Slope transition occurs at $1.25 \pm 0.15$ for a Stokes Number of 5.0 and a Froude Number of 1.0.

Crossing these transitions has the following effects on the WSS on the bottom of the cylinder:

- At the Stokes transition, there is a localized increase in the slope of the WSS.

- The Froude transition results in a localized increase in the slope of the WSS.

- The Slope transition causes a localized increase in the slope of the WSS.

Additional transitions were observed for the studied cases.

- Within the range of Stokes Numbers investigated, an effect resembling a Froude transition occurs at a Stokes Number of $2.5 \pm 0.25$ for a Froude number of 1.0 and a Slope Ratio of 1.0.

- Also within the range of Stokes Numbers examined, an effect resembling a Slope Transition occurs at Stokes Number of 7.5 \pm 2.5 for a Froude Number of 1.0 and a Slope Ratio of 1.0. 
- In the range of Slope Ratios evaluated, an effect resembling a Stokes Transition is seen at Slope Ratio of $0.65 \pm 0.15$ for a Stokes Number of 5.0 and a Froude Number of 1.0.

- In the range of Slope Ratios evaluated, an effect resembling a Froude transition is seen at Slope Ratio of $0.85 \pm 0.15$ for a Stokes Number of 5.0 and a Froude Number of 1.0.

- In the range of Froude Numbers studied, effects resembling transitions for the Stokes Number and the Slope Ratio are not present. 


\section{RECOMMENDATIONS}

A large change in free surface plots develops between a Stokes Number of 5.0 and 10. It would be useful to see several more cases between these values to see how these differences unfold and to find the slope transition with greater accuracy.

This study investigated the seven points on the cener of the six faces and in the center of the 27 point cube in Figure 2. To fully quantify how variations of the three dimensionless parameters affect transitions and resulting WSS, it is desirable for the remaining 20 points to be investigated with further research.

Additional research should also use particle image velocimetry (PIV) techniques to validate the data obtained in FLUENT. The PIV system employs two cameras that work in parallel to create a three dimensional image inside the dish. Associated software analyzes the change in images a small time step apart to develop velocity and shear profiles. 


\section{REFERENCES CITED}

Anderson, W., Thomas, J., and Rumsey, C. 1984. Application of Thin Layer Navier Stokes Equations Near Maximum Lift, AIAA 84--0049, AIAA 22nd Aerospace science Meeting, Reno, Nevada.

Banerjee, R., Back, L., Back, M., and Cho, YI. 2000. Physiological flow simulation in residual human stenoses after coronary angioplasty. Journal of Biomechanical Engineering. 122:310-320.

Bird, R., Stewart, W., and Lightfoot, E. 2002. Transport Phenomena. New York: John Wiley \& Sons, Inc. 12.

Brown, D. and Lambert, A. 2004. Oxford Dictionary of National Biography. Oxford:

Oxford University Press.

Dardik, A., Chen, L., Frattini, J., Asada, H., Aziz, F., Kudo, F., and Sumpio, B. 2005. Differential effects of orbital and laminar shear stress on endothelial cells. Journal of Vascular Surgery. 41:869-880.

Elias, C., Desai, R., Patole, M., Joshi, J., and Mashelkar, R. 1995. Turbulent Shear StressEffect on Mammalian Cell Culture and Measurement Using Laser Doppler Anemometer. Chemical Engineering Science. 50:2431-2440.

Finney, R., Weir, M., Giordano, F. 2003. Thomas's Calculus Updated Tenth Edition. Boston:Addison Wesley. 2.

FLUENT version 6.2.5. ANSYS, Inc. Canonsburg, Pennsylvania.

Haga, M., Yamashita, A., Paszkowiak, J., Sumpio, B., and Dardik, A. 2003. Oscillatory shear stress increases smooth muscle cell proliferation and Akt phosphorylation. Journal of Vascular Surgery. 37:1277-1284.

Hinds, W. 1998. Aerosol Technology: Properties, Behavior and Measurement of Airborne Particles, Second Edition. New York: John Wiley \& Sons, Inc.

Kraiss, L., Alto, N., Dixon, D., McIntyre, T., Weyrich, A., and Zimmerman, G. 2003. Fluid flow regulates E-selectin protein levels in human endothelial cells by inhibiting translation. J-Vasc-Surg. 37:161-168

Kraiss, L., Weyrich, A., Alto, N., Dixon, D., Ennis, T., Modur, V., McIntyre, T., Prescott, S., and Zimmerman, G. 2000. Fluid flow activates a regulator of translation, p70/p85 S6 kinase, in human endothelial cells. American Journal of Physiology. 278:1537-1544. 
Levesque, M., and Nerem, R. 1985. The Elongation and Orientation of Cultured Cells in Response to Shear Stress. Journal of Biomedical Engineering. 107:341-347.

Ley, K., Lundgren, E., Berger, E., and Arfors, K. 1989. Shear-Dependant Inhibition of Granulocyte Adhesion to Cultured Endothelium by Dextran Sulfate. Blood. 73:1324-1330.

Marshall, I., Zhao, S., Papathanasopoulou, P., Hoskins, P., and Xu, X. 2004. MRI and CFD studies of pulsatile flow in healthy and stenosed carotid bifurication models. Journal of Biomechanics. 37:679-687.

McCabe, W., Smith, J., and Harriot, P. 2001. Unit Operations of Chemical Engineering. New York: McGraw Hill. 45-46.

Papathanasopoulou, P., Zhao, S., Köhler, U., Robertson, M., Long, Q., Hoskins, P., Xu, X., and Marshall, I. 2003. MRI measurement of time-resolved wall shear stress vectors in a carotid bifurcation model, and comparison with CFD predictions. Journal of Magnetic Resonance Imaging. 17:153-162.

Rance, P. and Warren, N. 1968. The inception movement of course material is oscillatory flow. Proceedings of the $11^{\text {th }}$ Conference in Costal Engineering. 487-491.

Saarinen, M., and Murhammer, D. 2000. Culture in the Rotating-Wall Vessel Affects Recombination Protein Production Capacity of Two Insect Cell Lines in Different Manners. In Vitro Cellular \& Developmental Biology - Animal. 36: 362-366.

Sakurai, A., Nakano, A., Yamaguchi, T., Masuda, M., Fujiwara, K. 1991. A computational fluid mechanical study of flow over cultured endothelial cells. Advances in Bioengineering. 20:299-302.

Satcher, R., Bussolari, S., Gimbrone, M., and Dewey, C. 1992. The distribution of fluid forces on model arterial endothelium using computational fluid dynamics. Journal of Biomechanical Engineering. 114:309-316.

Sharp, K. 2006. Stokes $2^{\text {nd }}$ problem extended to orbital motion of the wall. J. B. Speed School of Engineering, University of Louisville.

Sterpetti, A., Cucina, A., Morena, A., Di Donna, S., D'Angelo, L., Cavalarro, A., and Tipa, S. 1993. Shear stress increases the release of interleukin-1 and interleukin-6 by aortic endothelial cells. Surgery. 114:911-914.

Ueba, H., Kawakami, M., and Yaginuma, T. 1997. Shear Stress as an Inhibitor of Vascular Smooth Muscle Cell Proliferation: Role of Transforming Growth Factor-\$1 and Tissue-Type Plasminogen Activator. Arteriosclerosis, Thrombosis, and Vascular Biology. 17:1512-1516. 
Weston, S., Wood, N., Tabor, G., Gosman, A., and Firmin, D. 1998. Combined MRI and CFD analysis of fully developed steady and pulsatile laminar flow through a bend. Journal of Magnetic Resonance Imaging. 8:1158-1171.

Wilson, David B. 1987. Kelvin and Stokes A Comparative Study in Victorian Physics.

Yamaguchi, T., Hoshai, K., Okino, H., Sakurai, A., Hanai, S., Masuda, T., and Fujiwara, K. 1993. Shear stress distribution over confluently cultured endothelial cells. Bioengineering Conference. 24:167-170.

You, Z. 1999. The inception of sheet flow in oscillatory flow. Ocean Engineering. 26:227-285. 


\section{APPENDIX - COMPUTER PROGRAMS AND ALGORITHMS}

\section{A. Algorithm for Determining Projected Intervals}

A test was performed on a cylinder with a radius of $1.75 \mathrm{~cm}$ and a height of 0.8 $\mathrm{cm}$ in FLUENT to find optimum projected intervals. The results showed that 50 projected intervals resulted in 297050 cells and produced the best combination of fast convergence and accurate results. This base case is used to scale the ideal projected interval to any case. An iterative method is used to find the projected interval at a given set of cylinder dimensions. First, the number of cells on the face is calculated by multiplying the number of cells on the face of the base case (5941) by the radius of the new cylinder, dividing by the radius of the base case, and finally multiplying by a scale factor. The scale factor is an arbitrary value that is used for iteration. Next, the number of projected intervals is calculated by multiplying the number of projected intervals in the base case by the height of the new cylinder, dividing by the height of the base case and multiplying by the arbitrary scale factor. The number of cells in the cylinder is then calculated by multiplying the number of cells in the face by the number of projected intervals. The correct number of projected intervals for the cylinder is determined by varying the scale factor until the number of cells reach 300,000 .

\section{B. Example User Defined Function}

To insert motion into a case in FLUENT, a $\mathrm{C}$ language based program is used. For the orbital dish system of study, the code seen in Figure 108 is used to add oscillatory motion to the cylinder. The real radius in line six corresponds to the orbital radius, while $\mathrm{w}$ in line seven is the orbital speed. 


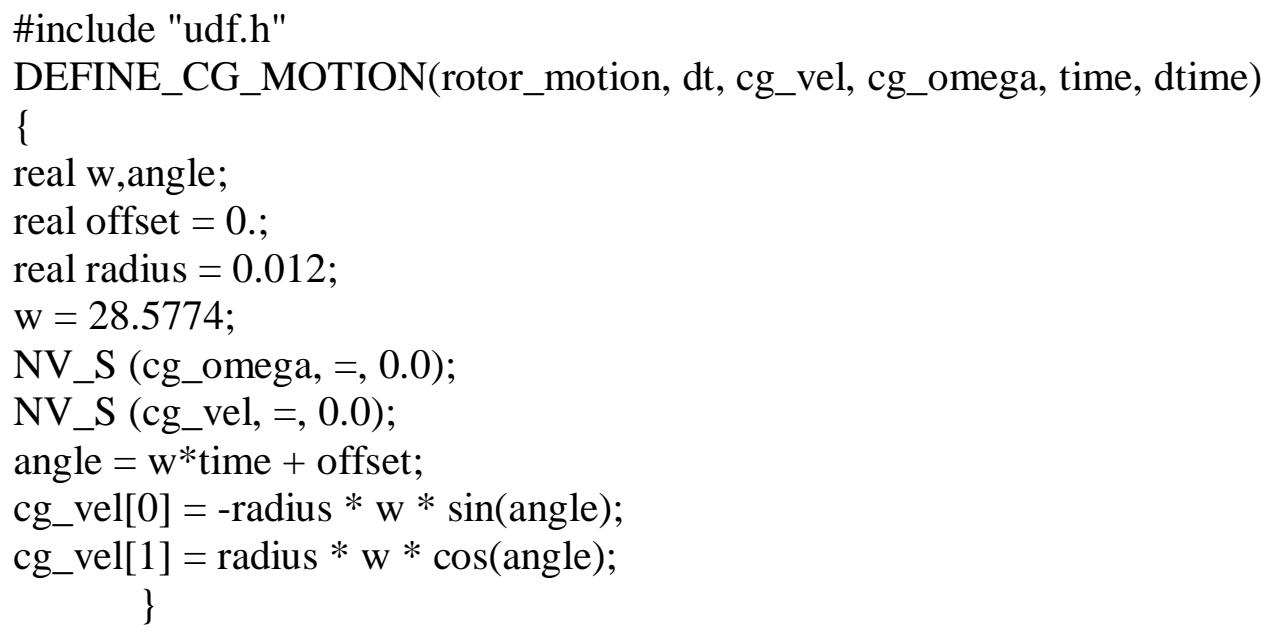

FIGURE 112 - Example UDF

\section{Example Batch File}

A batch file is used is need to run a case on a node of Adelie. The batch file opens FLUENT, tells the server what nodes to run on, and sets the location of the inputfile and the outputfile. Line three tells the number of nodes to use, line five sends an e-mail upon case completion, and line eight tells FLUENT to open the 3D version.

\#!/bin/bash

\#PBS -q dualcore2

\#PBS -1 nodes $=1: p p n=1$

\#PBS -m e

\#PBS -M jmthom27@louisville.edu

INPUT_FILE=/scr/JMDT/thesis/stokes/stokes10/inputfile_uss

OUTPUT_FILE=/scr/JMDT/thesis/stokes/stokes10/outputfile

$\mathrm{DIM}=3 \mathrm{~d}$

PROG="/apps/Fluent.Inc/bin/fluent "

PROGARGS="\$DIM -g -i \$INPUT_FILE"

echo Running on:

cat \$PBS_NODEFILE

NPROCS=`wc $-1<$ \$PBS_NODEFILE'

echo This job uses \$NPROCS processors

hostname

\$PROG \$PROGARGS > \$OUTPUT_FILE 2>\&1

FIGURE 113 - Example Batch File 


\section{Example Input File}

The input file tells FLUENT where to open the case and data files, how to solve the case, and where to write the results. Lines one and two tell FLUENT where to read the initial case and data file. Line five tells FLUENT the number of time steps. Line six gives the maximum number of iteration per time step. Lines seven and eight tell FLUENT where to write the results once iterations are complete.

rc/scr/JMDT/thesis/stokes/stokes10/10stokes5.cas $\mathrm{rd} / \mathrm{scr} / \mathrm{JMDT} /$ thesis/stokes/stokes10/10stokes5.dat solve

d

859

50

wc /scr/JMDT/thesis/stokes/stokes10/10stokes0864.cas

$\mathrm{wd} / \mathrm{scr} / \mathrm{JMDT} /$ thesis/stokes/stokes10/10stokes0864.dat

quit

exit

yes

FIGURE 114 - Example Input File 


\section{VITA}

Jonathan Michael D Thomas was born on July 12, 1984 in Louisville, Kentucky. He has lived in Louisville throughout his life, graduating from Southern High School in 2002 and graduating with a Bachelors Degree in Chemical Engineering from the University of Louisville in 2006. Jonathan's most favorite pastime is music, playing the guitar, bass, piano, trumpet, and mandolin. 